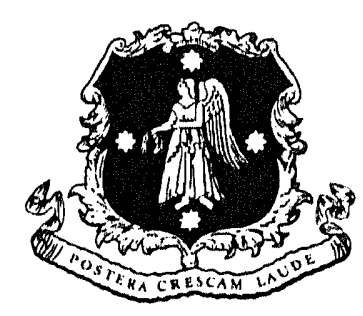

The University of Melbourne

Department of Mechanical and Manufacturing Enginecring

\title{
Wake structure of a transversely rotating sphere at moderate Reynolds numbers
}

\author{
By MATTEO GIACOBELLO
}

Submitted in total fulfilment of the requirements of the degree of Doctor of Philosophy

Septomber 2005 


\section{Abstract}

Over the last contury, the problem of a viscous flow past a sphere has received ongoing attention duo to its many enginecring applications. Theso include combustion processes, sediment transport processes and atmospheric flow problems, where the sphere scrves as a good model for more gencral bluff body particles. In these environments, particles may be subjected to both translational and rotational velocilies. The objective of this study was to investigate the effect that sphere rotation, about an axis transverse to the frecstrean flow, has on the characteristics of the vortical wake structure and the forces exerted on the sphere. This was achieved by solving the time-dependent, incompressible Navier Stokes exuations, using a highly accurato Fourier Cheloyshev spectral collocation method.

The sconario of stcady transverse rotation and a sinusoidally varying rotation rate were investigated. For the case of steady rotation, Reynolds numbers of $R e^{\prime}=100,250$ and 300 were considered, which, for a stationary sphore, cover the axisymmetrie steady, asymmetrie steady and vortex shedding regimes. The primary objective was to identify wake transitions that occur over the range of rotational specels $\Omega 2^{*}=0.00 \mathrm{t}, 01.00$, where $S 2^{*}$ is the maximum velocity on the sphere surface normalized by the frecstream velocity. For $R e=100$, stcaly rotation triggers a transition to a stcady clouble-thread structure. For $R e=250$, the wake undergoes a transition to vortex shedding for $\Omega^{*} \geq 0$. ()8. With increasing rotation rate, the recirculating region is progressively reduced until a further transition to a stcady double-thread wake structure for $\Omega^{*} \geq 0.30$. For $R e=300$, wake shedding is suppressed for $\Omega^{*} \geq 0.50$ via the same mechanism found for $R e=250$. Sphere rotation results in a thinning of the shear layer over one side of the sphere. For 
$\Omega^{*} \geq 0.80$, the wake undergoes a further transition to vortex shedding, through what appcars to be a shear laycr instability of the Kelvin- Helmholtz type.

The effect of oscillatory transverse rotation was considered for a Reynolds number of 300, a peak non-dimensional angular velocity of $\Omega_{\circ}^{*}=0.50$ and a range of forcing frequencics that encompass the natural shedding frequency. Oscillatory forcing leards 1,0 at two-sided shedding of alternatcly signed hairpin structures. Over the forcing freculency range considered, the wake shedding becomes synchronized with the forcing frequency. For forcing frequencies below that of natural vortex shedding, there is a wiclening of the wake, while for higher frequencies the width of the wake is progressively reduced. As the forcing frequency is increased, wake synchronization dictates that vortex loops are formed and released at a faster rate. This results in a greater strcamwise and latcral packing of consecutively shed structures. 


\section{Declarations}

This is to cortify that

1. the thesis comprises only my original work towards the PhD,

2. due acknowledgment has been made in the text to all other material used,

3. The thesis is less than 100000) words in length, exclusive of tables, maps, bibliographies and appendices.

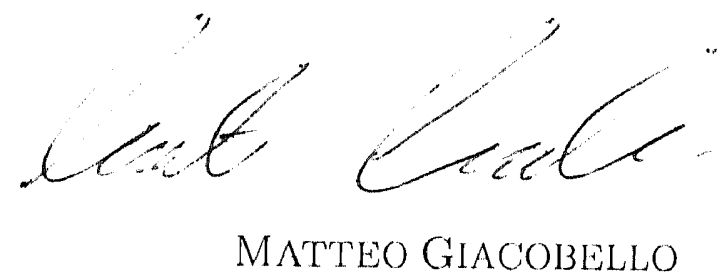




\section{Acknowledgements}

I would like to thank my supervisor Dr. $\Lambda$. Ooi for his guidance. His enthusiasm for fluid dynamics and applied mathematies is infections and I am very gratoful to have had the opportunity to loarn from him.

Prof. M. S. Chong was a constant source of inspiration and helpful criticism. I thank him for his interest and readiness to discuss my work. Thank-you also for the lunch time discussions on munerous fluid dynemics problems.

I woukd like to thank Prof. S. Balachandar for his export advice regarding blufl body fluid dynamics and also for providing me with the computer code that formed the basis of this project.

I thank my past and present colleagues for valuable discussions, heir friendship and for making the 'fluids lab)' a fantastic place to study: Mr. P. Gregory, Dr. K. Higgins, Dr. M. B. Jonces, Dr. J. Monly, Mr. N. Nishizawa, Dr. S. Haffez, Dr. A. K. Hollstedt, Mr. R. Widjaja, Ms. K. Lien, Mr. R. Cohen and Mr. K. Szczopanik.

Special thanks goes to Dr. K. Higgins for bringing me up to speed with numerical lechnicgnes. I thank him for many invaluable discussions and suggestions not only on the topice of fluid mechanics. Special thanks also to Mr. R. Widjaja for many discussions on the subject of numerical methods.

Thank-you to Dr. J. Monty, for his friendship, for always been willing to help me solve 
my many software and hardware problems, and for vetting this manuscript. Thankyou also to Ms. K. Lien for vetting this manuscript and for many thought provoking discussions.

Thank-you to Mr. D. Jaquest, for his friendship and great advice on D.I.Y. home improvement projects.

I express my gratitude to the Air Vehicles Division (AVD) of the Defence Science and Tochnology organization (DSTO) for financially supporting my candidature. In particular I thank Dr. B. Fairlic, Dr. D. Thompson, Dr. N. Matheson, Dr. B. Baskaran, Mr. J. Drobik, Dr. C. Guy, Mr. N. Pollock and Mr. B. Woodyatt for approving my application to go back to school. Spccial thanks go to Mr. J. Drobik for his continual support over the course of my candidature.

Thank-you to Prof. K. Dongjoo and Prof. H. Choi for providing figures for the validation of the code used in this study. Thank-you also to Prof. T. Lewcke and Prof. M. Thompson for providing their high quality flow visualization images and details of the experiment.

Many thanks go to the Victorian Partnership for Advaneed Computing (VP $\Lambda($ ) and the Australian Partnership for Advanced Computing ( $\mathrm{P} \wedge \mathrm{C})$ for providing the computing resources that, were essential to this project.

To my family, I swe the greatest debt. The Ph.D. process is a very self indulgent one and family and friends were often neglected. I thank them for their paticnec, support and encouragemont.

Mattco Giacobello 
DEDICATO A MIA MADRE 


\section{Contents}

Abstract iii

Declarations $\quad \mathrm{v}$

Acknowledgements vii

Contents $x$

List of Figures $\quad x v$

List of Tables $\quad$ xxi

$\begin{array}{ll}\text { Nomenclature } & \text { xxiii }\end{array}$

1 Literature review 1

1.1 The uniform flow past a stationary sphere . . . . . . . . . . . 3

1.2 The flow past a spinning sphere . . . . . . . . . . 17

1.3 The flow past a cylinder in rotary oscillation . . . . . . . . . 29

1.4 Ains and outline . . . . . . . . . . . . . . . . . 39

2 Numerical formulation 41

2.1 Governing equations and coordinate system . . . . . . . . . 41

2.2 Spatial discretization . . . . . . . . . . . . . . . . . 45

2.2 .1 The pole problem . . . . . . . . . . . . . . . 50

2.3 Spatial differentiation . . . . . . . . . . . . . . 52 
2.3.1 Radial derivatives . . . . . . . . . . . . . . 53

2.3.2 Tangential derivatives $\ldots \ldots \ldots \ldots \ldots 5$

2.4 Temporal discretization . . . . . . . . . . . . . 56

2.4.1 Fractional-stcp method I (Fsm I) . . . . . . . . . . . 60

2.4.2 Fractional-stcp method II (Fsm II) . . . . . . . . . . 60

2.4.3 Fractional-step method III (Fsm III) . . . . . . . . . 61

2.5 Velocity boundary conditions . . . . . . . . . . . 62

2.5.1 Far-ficld boundary conditions ............. 62

2.5 .2 Surface boundary conditions . . . . . . . . . . 63

2.6 Initial conditions . . . . . . . . . . . . . . . 64

2.7 Solution algorithm $\ldots \ldots \ldots \ldots \ldots \ldots$

3 Code validation $\quad 71$

3.1 Evaluation of the fractional-step methods. . . . . . . . . . . . 72

3.1.1 A Neumann boundary condition for the wall-normal velocity . . 76

3.2 Scnsitivity to spatial and tomporal resolution . . . . . . . . . . 80 80

3.3 Performance of the outflow boundary condition . . . . . . . . 85

3.4 Comparison with carlicr studics . . . . . . . . . . . . . 89

3.1 Axisymmetric reginc, $20<R c<212 \ldots \ldots \ldots$

3.1 .2 Asymmotric stcady regime, $212<$ Re $<270 \ldots \ldots$. . . . . 95

3.4 .3 Vortex shedding regime, $R c>270 \ldots \ldots \ldots 9 . \ldots \ldots$

4 Vortical structure identification $\quad 101$

4.1 The discriminant elefinition of a vortex . . . . . . . . . . . 10):3

4.2 The $Q_{A}$ definition of a vortex . . . . . . . . . . . . 106

4.3 The $\lambda_{2}$ chefinition of a vortex . . . . . . . . . . . . . . . 107

4.4 The swirling strength definition of a vortex . . . . . . . . . . . 108

4.5 A critical cvaluation . . . . . . . . . . . . . . . 109

4.5.1 Comparative application to the flow past a sphere . . . . . . . 112 
5 Flow past a sphere undergoing steady transverse rotation $\quad 117$

5.1 Vortical wake structure . . . . . . . . . . . . . . . . 119

5.2 Near-wake strcamline pattern . . . . . . . . . . . . . . 141

5.3 Vorticity distribution . . . . . . . . . . . . . 166

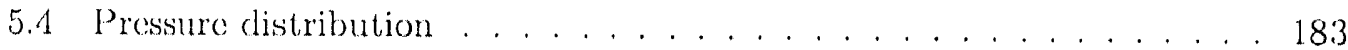

5.5 Forece coefficient histories . . . . . . . . . . . . . . . 197

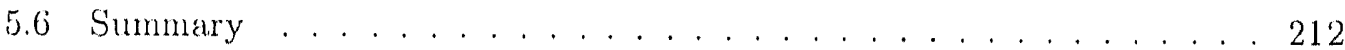

6 Flow past a sphere undergoing oscillatory transverse rotation 215

6.1 Forec coeflicient historiess . . . . . . . . . . . . . . . . 217

6.2 Vortical wake structure . . . . . . . . . . . . . . . 225

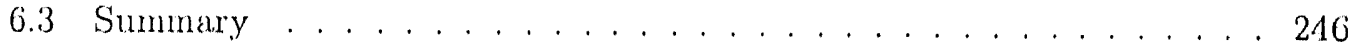

$\begin{array}{lll}7 & \text { Conclusions } & 247\end{array}$

7.1 Suggestions for future work . . . . . . . . . . . . . . 250

$\begin{array}{lr}\text { References } & 253\end{array}$

$\begin{array}{ll}\text { A Fourier series on spheres } & 265\end{array}$

A.1 Basis functions for a scalar variable . . . . . . . . . . . 266

A.2 Basis functions for a vector variable . . . . . . . . . . . 269

B Instantancous streamline patterns on the $(x, z)$-plane 275

C The velocity boundary condition on a spinning sphere 279 


\section{List of Figures}

1.1 Drag coefficient versus Reynolds number for a smooth sphere . . . . . . 3

1.2 Flow visualization of the sphere wake using aluminium dust in water . 5

1.3 Flow visualization of the sphere wake structure at $R e=270 \ldots \ldots$

1.4 Flow visualization of the sphere wake at $R e=320 \ldots \ldots$. . . . . .

1.5 Vortex configuration in the wake of a sphere at $R e=1000 \ldots \ldots 10$

1.6 Instantaneous vortical structure computed for Re $=300 \ldots \ldots \ldots 11$

1.7 Strouhal number versus Rcynolds number . . . . . . . . . . . 14

1.8 Definition of the advancing and retreating sides of a rotating sphere . . 18

1.9 Time-averaged lift coefficient, $\overline{C_{L}}$, versus $\Omega^{*} \ldots \ldots \ldots 21$

1.10) Timo-averaged drag coefficiont, $\overline{C_{D}}$, versus $\Omega^{*} \ldots \ldots \ldots 22$

1.11 Flow visualization of a rotating sphere at $R e \approx 140 \ldots \ldots 26$

1.12 Schomatic of the lock-on band . . . . . . . . . . . . . 31

1.13 The wake of a cylinder in rotary oscillation at $R e=1.5 \times 10^{4} \ldots 37$

2.1 Prollem geometry and coordinate system . . . . . . . . . . . 4 44

2.2 Stretching functions used in the radial and tangential clirections . . . 49

$2.3 \wedge$ representative computational grid . . . . . . . . . . . 50

2.4 Collocation point spacing in the vicinity of the sphere poles . . . . . . 51

2.5 Pole filter, $g_{\phi}$, versus collocation point spacing, $Y \ldots \ldots . \ldots . \ldots 5$

2.6 Non-reflective filter function, $g(r, \theta)$, and its first derivatives $\ldots . .64$

3.1 Pressurc drag versus $\Delta t^{*}$ for $R e=100 \ldots \ldots \ldots 74$ 
3.2 Pressure cocfficient along a ray near the equator for $R e=100 \ldots$. . . 74

3.3 Radial velocity along a ray ncar the equator for $R e=100 \ldots \ldots . .75$

3.4 Pressure coefficient along a ray near the equator for $R e=100 \ldots$. . . 78

3.5 Radial velocity along a ray near the equator for $R e=100 \ldots$. . . . 79

3.6 Effect of $N_{\phi}$ on the vortical wake structure for $R e=300 \ldots \ldots$

3.7 Instantancous spectral modes of velocity for $R e=300 \ldots \ldots 4$

$3.8 x$-vorticity for a discrete structure convecting downstroam . . . . . . 87

$3.9 x$-vorticity along $\theta=0.011 \pi$ for a discrete structure convecting down-

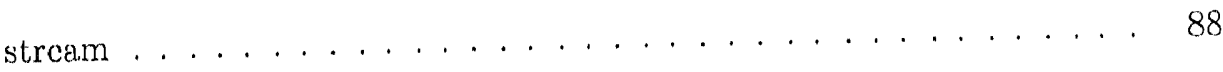

3.10 Separation angle and wake length with respect to Re . . . . . . . 91

3.11 Drag cocficient with respect to Re . . . . . . . . . . . . 92

3.12 Base prossure coefficient with respect to Re . . . . . . . . . . 93

3.13 Drag coclficiont al $R e=100$ with respect to $\Omega^{*} \ldots \ldots . \ldots 3$

3.14 Separation angle and wake length with respect to $\Omega^{*}$ for $R e=100 \ldots$. 94

3.15 Vorticity contours for $R e=250 \ldots \ldots \ldots$

3.16 Vortical structures for $R e=250 \ldots \ldots \ldots 7$

3.17 Forec historics for streamwise rolation at $R e=250$ and $\Omega^{*}=0.10 .98$

3.18 Vortical structures at $R e=300 \ldots \ldots \ldots$. . . . . . . . . . . .

4.1 Local non-degenerate topologies for incompressible flows . . . . . . 105

4.2 Stroanline pattern in the vicinity of a canonical critical point . . . . 111

4.3 lsot)-surfaces of $\omega_{z},|\omega|$ and $Q_{A}$ for $R e=300 \ldots \ldots \ldots \ldots . \ldots \ldots$

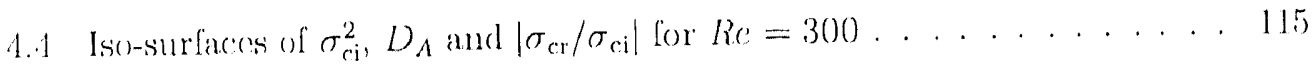

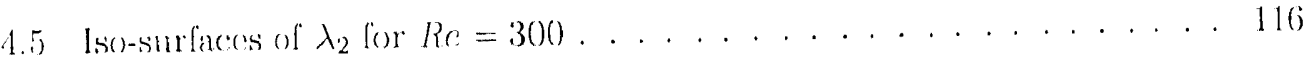

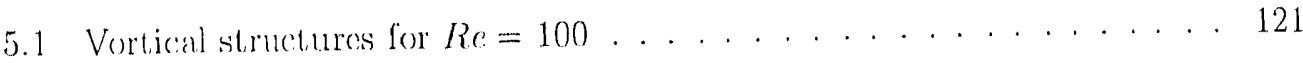

5.2 Instantaneous vortical structures for $R e=250 \ldots \ldots \ldots \ldots$

5.3 Evolution of the vortical structure for $R e=250$ and $\Omega^{*}=0.10 \ldots \ldots$

5.4 Evolution of the vortical structure for $R e=250$ and $\Omega^{*}=0.20 \ldots \ldots$ 
5.5 Instantancous vortical structures for $R e=300 \ldots \ldots$. . . . . . 129

5.6 Evolution of the vortical structure for $R e=300$ and $\Omega^{*}=0.00 \ldots 135$

5.7 Evolution of the vortical structure for $R e=300$ and $\Omega^{*}=0.30 \ldots 136$

5.8 Evolution of the vortical structure for $R e=300$ and $\Omega^{*}=0.80 \ldots 137$

5.9 Effect of symmetry breaking perturbation for $R e=300$ and $\Omega^{*}=1.00 .138$

5.10 Time-history of azimuthal mode $k=0$ of $u_{\phi} \ldots \ldots$. . . . . . . . . 139

5.11 Vortical structure derived from the timc-averaged velocity field . . . . . 140

5.12 Streamline patterns on the $(x, z)$-plane for $R e=100 \ldots \ldots \ldots \ldots$

5.13 Streamline patterns on the $(y, z)$-plane for $R e=100 \ldots \ldots$. . . . . 114

5.14 Threc-dimonsional streamline pattorns for $R e=100 \ldots \ldots \ldots$

5.15 Instantaneous stroamline pattorns on the $(y, z)$-plane for $R e=250 \ldots 149$

5.16 Threc-dimensional particle paths for a stationary sphere at $R c=250 \quad .150$

5.17 Streamline evolution on the $(y, z)$-plane for $R e=250$ and $\Omega^{*}=0.10, \ldots 151$

5.18 Streamline cvolution on the $(y, z)$-plane for $R e=250$ and $\Omega^{*}=0.20 \ldots 152$

5.19 Instantancous streamline patterns on the $(y, z)$-plane for Re $=300 \ldots 155$

5.20 Reverse flow region for $R e=100$ and $\Omega^{*}=0.30 \ldots \ldots 150$

5.21 Streamline evolution on the $(y, z)$-plane for $R e=300$ and $\Omega^{*}=0.00 \ldots 160$

5.22 Streamline evolution on the $(y, z)$-plane for $R e=300$ and $\Omega^{*}=0.30 \ldots 161$

5.23 Streanline evolution on the $(y, z)$-plane for $R e=300$ and $\Omega^{*}=0.80 ., 162$

5.24 Instantancons cross-stream streamline pattern at, $z=11.5 \ldots \ldots 3$

5.25 Profiles of $\overline{u_{z}}$ and $\overline{u_{y}}$ at $z=2.5$ for $R e=100,250$ and $300 \ldots \ldots 4$

5.26 Profiles of $\overline{u_{z}}$ and $\overline{u_{y}}$ at $z=2.5$ for $\Omega^{*}=0.00,0.30$ and $1.00 \ldots \ldots . .165$

5.27 Contours of $x$-vorticity on the $(y, z)$-plane for $R c=100 \ldots \ldots$

5.28 Vortex lines for $R e=100$ and $\Omega^{*}=1.00 \ldots \ldots \ldots$. . . . . . . .

5.29 Iso-surfaces of $z$-vorticity for $R c=100 \ldots \ldots \ldots 171$

5.30 Contours of $x$-vorticity on the $(y, z)$-plane for $R e=250 \ldots \ldots$

5.31 Iso-surfaces of instantencous $z$-vorticity for $R e=250 \ldots \ldots \ldots$

5.32 Contours of $x$-vorticity on the $(y, z)$-plane for $R e=300 \ldots \ldots \ldots$ 
5.33 Iso-surfaces of instantaneous $z$-vorticity for $R e=300 \ldots \ldots$. . . . . 180

5.34 Time-averaged surface $x$-vorticity on the $(y, z)$-plane $\ldots \ldots \ldots$

5.35 Pressure cocfficient contours on the $(x, z)$-plane for $R e=100 \ldots \ldots 185$

5.36 Pressure cocfficient contours on the $(y, z)$-plane for $R e=100 \ldots \ldots . .186$

5.37 Pressure coefficient along the sphere surface for $R e=100 \ldots \ldots$. . . 187

5.38 Pressure coefficient contours on the $(x, z)$-plane for $R e=250 \ldots \ldots . .192$

5.39 Pressure coefficient contours on the $(y, z)$-plane for Re $=250 \ldots \ldots . .193$

5.40 Pressure coefficient contours on the $(x, z)$-plane for Re $=300 \ldots \ldots . .194$

5.41 Pressure coefficient contours on the $(y, z)$-planc for $R e=300 \ldots \ldots . .195$

5.42 Time-averaged pressure coefficient along the sphere surface . . . . . . 196

5.43 Time-historics of the drag and lift coefficients for $R e=100 \ldots \ldots$. . . 199

5.44 Time-histories of the drag and lift cocfficients for $R e=250 \ldots \ldots$. . 200)

5.45 Time-histories of the drag and lift coefficients for $R e=300 \ldots \ldots . .201$

5.46 Vort,cx shedding Strouhal number, $S t_{v}$, versus $\Omega^{*} \ldots \ldots \ldots . . \ldots 202$

5.47 Drag and lift cocflicients versus $\Omega^{*}$ for $R c=100 \ldots \ldots$ 205

5.48 Timc-averaged clrag and lift coefficients vorsus $\Omega^{*}$ for $R e=250 \ldots .206$

5.19 Time-averaged drag and lift coefficients vorsus $\Omega^{*}$ for Re $=300 \ldots . .207$

5.50 Volocity vectors on the surface of the sphere . . . . . . . . . . . 208

5.51 Time-averaged total drag and total lift cocflicionts versus $\Omega^{*} \ldots \ldots$. . 210

5.52 Time-averaged total lift wo total drag ratio versus $\Omega^{*} \ldots \ldots \ldots . . . .211$

5.53 Time-averaged torque coeflicient versus $\Omega^{*} \ldots \ldots \ldots . \ldots . \ldots . \ldots 211$

6.1 Time-histories of the lift and drag coefficients . . . . . . . . 221

6.2 Phase diagram of $C_{D}$ versus $C_{L, y}$ for various $S t_{f} / S t_{n} \ldots \ldots \ldots . \ldots . . \ldots 22$

6.3 Vortex shexdling frecuency ratio versus the forcing frequency ratio . . . 223

6.4 Phase angle, $\Delta \psi$, versus the forcing frequency ratio . . . . . . . . 223

6.5 Time-averaged and fluctuating force coefficients . . . . . . . . . 224

0.6 Isc-surfaces of instantancous $z$-vorticity for various $S t_{f} / S t_{n} \ldots \ldots .226$ 
6.7 Contours of time-averaged $x$-vorticity on the $(y, z)$-plane $\ldots \ldots \ldots 227$

6.8 Evolution of the vortical structure for $S t_{f} / S t_{n}=0.25 \ldots \ldots 235$

6.9 Evolution of the vortical structure for $S t_{f} / S t_{n}=0.50 \ldots 237$

6.10) Evolution of the vortical structure for $S t_{f} / S t_{n}=1.00 \ldots \ldots 238$

6.11 Evolution of the vortical structure for $S t_{f} / S t_{n}=1.50 \ldots \ldots 239$

6.12 Evolution of the vortical structure for $S t_{f} / S t_{n}=2.00 \ldots \ldots$. . . . 240

6.13 Evolution of the vortical structure for $S t_{s} / S t_{n}=2.50 \ldots 241$

6.14 Evolution of the vortical structure for $S t_{s} / S t_{n}=3.00 \ldots 242$

6.15 Closc-up of vortical near-wake structure for $S t_{f} / S t_{n}=3.00 \ldots 243$

6.16 Profiles of $\overline{u_{z}}$ at $z=2.5$ and $z=5.0$ for various $S t_{f} / S t_{n} \ldots \ldots 244$

6.17 Profiles of $\overline{u_{z}}$ along the downstream pole for various $S t_{f} / S t_{n} \ldots \ldots 245$

6.18 Near-wake strcamwise velocity time-histories for $S \iota_{f} / S t_{n}=0.25 \ldots 245$

A.1 Typical even and odd azimuthal modes in the vicinity of a pole . . . 268

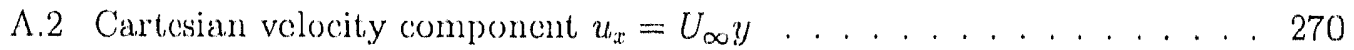

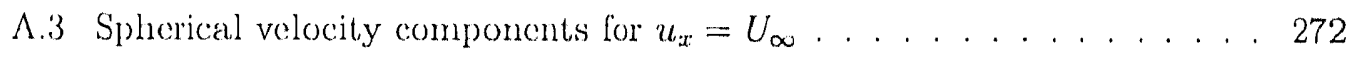

A.4 Spherical velocity components for $u_{x}=U_{\infty} y \ldots \ldots . \ldots . \ldots 273$

B.1 Instantancous streamline pattems on the $(x, z)$-plane for $R e=250 \ldots 276$

B.2 Instantaneous streanline paticens on the $(x, z)$-plane for $R e=300 \ldots .277$

C.1 Coordinate system omployed by Kurose \& Komori (1999) and Niazmand \& Ronksizbulut (2003a) . . . . . . . . . . . . . 279 


\section{List of Tables}

3.1 Dirichlet versus Neumann boundary condition for $u_{r}^{\star} \ldots \ldots 78$

3.2 Sonsitivity to $\Delta t^{*}$ for $R e=100 \ldots \ldots \ldots$. . . . . . . . 81

3.3 Sensitivity to $N_{r}$ for $R e=100 \ldots \ldots \ldots 1$

3.1 Sonsitivity to $N_{0}$ for $R e=100 \ldots \ldots \ldots 2$

3.5 Sensitivity to $D_{0}$ for $R e=100 \ldots \ldots \ldots 2$

3.6 Sonsitivity to $D_{0}$ for $R e=300 \ldots \ldots \ldots 2 \ldots \ldots \ldots$

3.7 Scnsilivity to $\Delta t^{*}$ for $R . c=300 \ldots \ldots \ldots \ldots 2 \ldots \ldots \ldots$

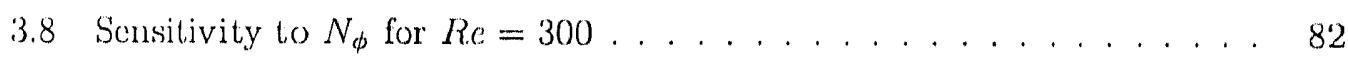

3.9 Comparison of $\overline{C_{D}}, \overline{C_{L}}$ and $S t_{n}$ with carlior numerical results . . . . . 99

5.1 Discrete values of non-dimensional rotation rate simulated . . . . . 118

5.2 Spatial and temporal simulation parameters . . . . . . . . . . 118

6.1 Spatial and temporal simulation parameters . . . . . . . . . . 217 


\section{Nomenclature}

\section{Roman symbols}

\begin{tabular}{|c|c|}
\hline$a$ & sphore radius \\
\hline$c_{0}, c_{1}, c_{2}$ & radial coordinate stretching function parameters \\
\hline$d$ & sphere diameter \\
\hline $\mathrm{cl} S$ & clement surface area \\
\hline$e_{i}$ & cigenvectors of the velocity gradient tonsor \\
\hline$\left(\widehat{e}_{r}, \widehat{e}_{\theta}, \widehat{e}_{\phi}\right)$ & unit vectors parallel to the spherical polar coorclinate lines \\
\hline$\left(\widehat{e}_{x}, \widehat{e}_{y}, \widehat{e}_{z}\right)$ & unit vectors parallel to the cartesian coordinate lines \\
\hline $\int_{f}$ & forcing frequency \\
\hline $\int_{m o d}$ & modulation frequency \\
\hline $\int_{n}$ & unforced vortex shedding frecquency \\
\hline $\int_{11}$ & vortex shedding frecuency \\
\hline$f\left(x_{j}\right)$ & value of a general function at the radial grid node $j$ \\
\hline$y_{\phi}(r, 0, k)$ & Fourier mode filter \\
\hline$g(r, \theta)$ & outflow boundary non-reflective filter function \\
\hline$i, j, l$ & index variables \\
\hline$n$ & denoting solution at the $n^{\text {th }}$ time-step \\
\hline$\widehat{n}$ & wall-normal unit vector \\
\hline$p, m, k$ & radial, tangential and azimuthal expansion wavenumbers, respectively \\
\hline$q$ & approximation to pressure in advection-diffusion equation \\
\hline$(r, \theta, z)$ & non-dimensional cylindrical polar coordinates \\
\hline
\end{tabular}




\begin{tabular}{|c|c|}
\hline$(r, \theta, \phi)$ & non-dimensional spherical polar coordinates \\
\hline$r_{i}$ & radial collocation point $i$ in the physical domain \\
\hline$s$ & scalar function \\
\hline$t$ & dimensional time \\
\hline$t^{*}$ & non-dimensional time, $t^{*}=t U_{\infty} / d$ \\
\hline$l_{c:}^{*}$ & $=t^{*}-t_{0}^{*}$ \\
\hline$t_{f}^{*}$ & non-dimensional final time \\
\hline$t_{o}^{*}$ & non-dimensional vortex shedding cycle start time \\
\hline$t_{s}^{*}$ & non-dimensional start time \\
\hline$u$ & non-dimensional velocity vector \\
\hline$u_{b}$ & non-dimonsional velocity boundary conditions \\
\hline$\left(u_{r}, u_{0}, u_{\phi}\right)$ & non-dimensional velocity components in spherical coordinates \\
\hline$\left(u_{x}, u_{y}, u_{z}\right)$ & non-dimensional velocity components in cartesian coordinates \\
\hline$(x, y, z)$ & non-dimensional cartesian coordinates \\
\hline$\left(x_{1}, x_{2}, x_{3}\right)$ & genoralized orthogonal curvilinear coordinates \\
\hline$x_{i}$ & Gauss-Lobatto collocation points on the natural domain \\
\hline$\tilde{x}_{i}$ & radial strctching function \\
\hline$x_{\text {min }}$ & radial coordinate stretching function parametcr \\
\hline$A_{i j}, \mathbf{A}$ & velocity gradient tonsor \\
\hline $\mathrm{B}$ & l. wansformation matrix \\
\hline$C_{1}, C_{2}, C_{3}$ & constants \\
\hline$C_{1}$ & $\operatorname{lrag}$ coeflicient, $C_{D}=D / \frac{1}{2} \rho U_{\infty}^{2} S$ \\
\hline$C_{L}$ & lilt coclicient, $C_{L}=L / \frac{1}{2} \rho U_{\infty}^{2} S$ \\
\hline$C_{M}$ & torgue coefliciont, $C_{M}^{4}=M / \frac{1}{2} \rho U_{\infty}^{2} S d$ \\
\hline$C_{\Gamma}$ & pressinte cocflicient, $C_{P}=\left(P-P_{\infty}\right) / \frac{1}{2} \rho U_{\infty}^{2}$ \\
\hline$C_{P b}$ & pressure coefficient at the sphere downstream pole \\
\hline $\mathcal{C}_{l j}^{1}$ & Fourier-cosine first-derivative operator matrix \\
\hline$D$ & dimensional drag \\
\hline
\end{tabular}




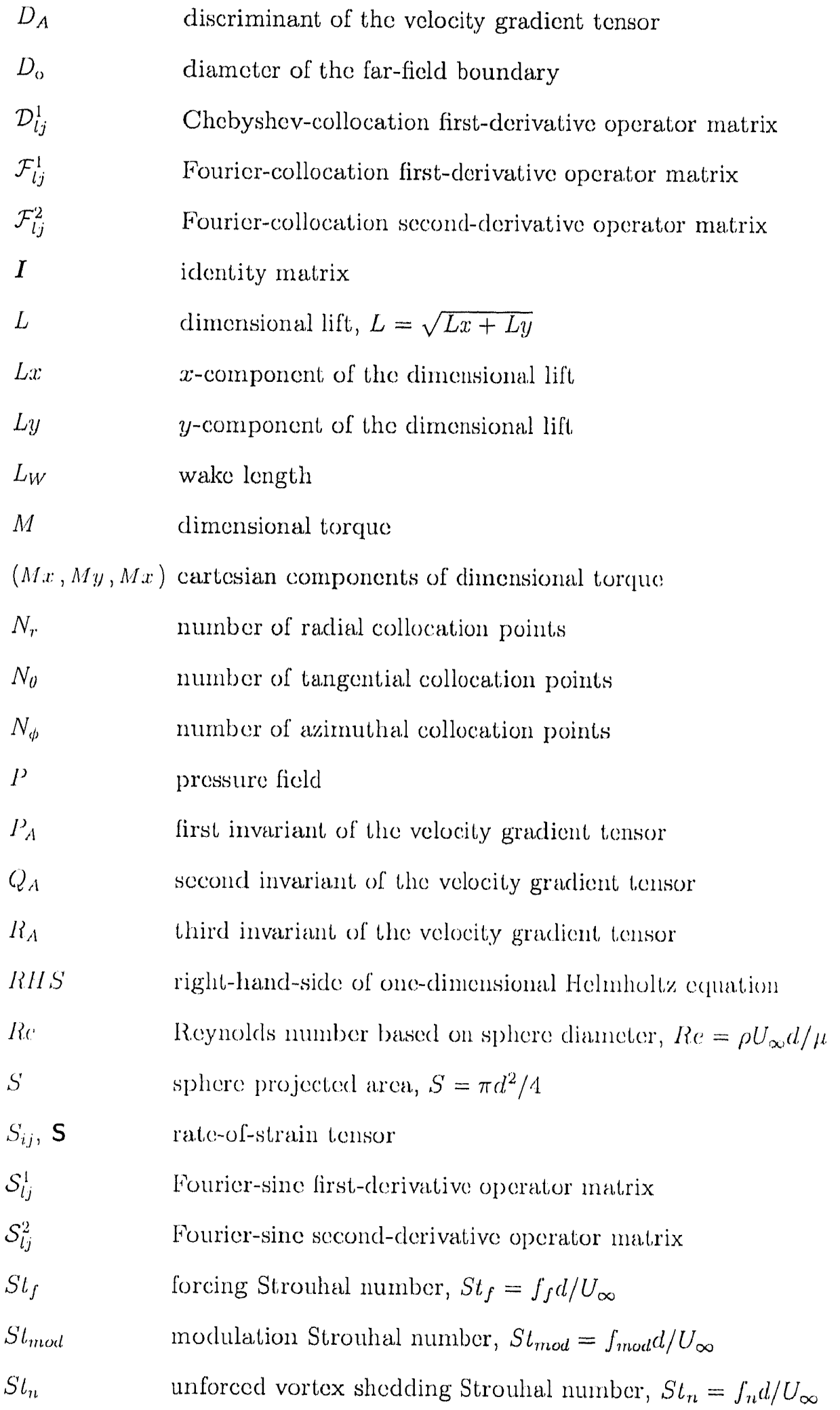




$\begin{array}{ll}S t_{v} & \text { vortex shedding Strouhal number, } S t_{v}=f_{v} d / U_{\infty} \\ T & \text { period of vortex shedding cycle } \\ T_{p}(r) & p^{\text {th }} \text { Chebyshev polynomial } \\ U_{\infty} & \text { freestream velocity magnitude } \\ V & \text { freestream velocity vector } \\ Y & \text { distance normal to the } z \text {-axis, } Y=r \sin \theta\end{array}$

\section{Greek symbols}

\begin{tabular}{|c|c|}
\hline$\alpha_{\circ}$ & peak angular accolcration of rotary oscillation \\
\hline$\alpha_{p m k}$ & expansion cocfficients for a scalar ficld \\
\hline$\beta_{p m k}$ & expansion coefficients for the radial velocity field \\
\hline$\gamma_{p m k}$ & expansion cocficients for the tangential velocity ficld \\
\hline$\delta_{p m k}$ & expansion coefficients for the azimuthal velocity ficld \\
\hline$\epsilon$ & machine procision \\
\hline$\zeta$ & constant in the non-reflective filter function $g(r, \theta)$ \\
\hline$\eta_{1}, \eta_{2}$ & constants in the non-reflective filter function $g(r, \theta)$ \\
\hline$\theta_{j}$ & tangential collocation point $j$ in the physical domain \\
\hline$\tilde{\theta}_{j}$ & tangential collocation points on the natural domain \\
\hline$\theta_{S}$ & flow separation anglo \\
\hline$\lambda_{1}, \lambda_{2}, \lambda_{3}$ & eigenvalues of the tensor $S_{k j} S_{i k}+S_{k j} \Omega_{i k}, \lambda_{1} \geq \lambda_{2} \geq \lambda_{3}$ \\
\hline$\mu$ & rlynamic viscessily \\
\hline$\nu$ & kinematice viscosity \\
\hline$\xi(k)$ & function in the Fourier mode filter $g_{\phi}(r, \theta, k)$ \\
\hline$\rho$ & dluid density \\
\hline$\sigma$ & stress tomsor \\
\hline$\sigma_{i}$ & cigenvalues of the velocity gradient tonsor \\
\hline$\sigma_{\mathrm{cr}}, \sigma_{\mathrm{ci}}$ & real and imaginary components of complex $\sigma_{i}$ \\
\hline$\widehat{\tau}$ & wall-parallel unit vector \\
\hline
\end{tabular}


$\phi_{k} \quad$ Fouricr-collocation point $k$ in the azimuthal direction

$\varphi(k) \quad$ function in the Fourier mode filter $g_{\phi}(r, \theta, k)$

$\psi_{f} \quad$ angular velocity phase angle

$\psi_{v} \quad$ vortex shedding phase angle

$\omega \quad$ vorticity vector

$\left(\omega_{x}, \omega_{y}, \omega_{z}\right) \quad$ cartesian components of the vorticity vector

(1) scalar variable lincarly related to the pressure field

$\Delta l \quad$ time-step

$\Delta \iota^{*} \quad$ non-dimensional time-step

$\Delta r \quad$ radial collocation point spacing

$\Delta \theta \quad$ tangential collocation point spacing

$\Delta \phi \quad$ azimuthal collocation point spacing

$\Delta \psi \quad$ phase difference, $\Delta \psi=\psi_{f}-\psi_{v}$

$\Theta_{0} \quad$ peak angular displacoment of rotary oscillation

$\Omega \quad$ dimensional rotation rate vector

$\Omega \quad$ dimensional rotation specd

$\Omega_{c} \quad \quad \quad$ critical value of $\Omega$ for vortex shedeling suppression

$\Omega_{0} \quad$ peak dimensional rotation rate for rotary oscillation

$\Omega_{i j}, \Omega \quad$ ratc-of-rotation tensor

\section{Other symbols}

$\mathbb{D}_{l j}^{1} \quad$ physical-space radial first-derivative operator matrix

$\mathbb{L} \quad$ linear operator in the fractional-step formulation

JI denoting the sphere surface

$\operatorname{tr}() \quad$ trace of a matrix

$\operatorname{det}($ ) determinant of a matrix

()$_{\infty} \quad$ denoting the value of a variable in the frecstream

()$_{P} \quad$ pressure component 


$\begin{array}{ll}()_{p p} & \text { peak-to-peak fluctuating amplitude } \\ ()_{v} & \text { viscous component } \\ ()^{T} & \text { denoting matrix transpose } \\ ()_{r} & r \text {-component of a vector expression } \\ ()_{\theta} & \theta \text {-component of a vector cxpression } \\ ()_{\phi} & \phi \text {-component of a vector expression } \\ ()^{\prime} & \text { denoting canonical form of a tensor } \\ ()^{*} & \text { denoting non-dimensional rotation rate } \\ ()^{*} & \text { denoting variable at the intermediate timc-step } \\ \widehat{()} & \text { Fouricr cocfficient } \\ (\bar{T} & \text { denoting a lime-averaged quantity } \\ {[]} & \text { denoting discretc matrix representation }\end{array}$

\section{Abbreviations}

\begin{tabular}{|c|c|}
\hline $\max$ & maximum \\
\hline $\min$ & minimum \\
\hline $\mathrm{rms}$ & root-mean-square \\
\hline$\Lambda \mathrm{DI}$ & Altcrnating Direction Implicit \\
\hline $\mathrm{CFL}_{4}$ & Courant, Fricdrichs and Lewy \\
\hline DNS & direct mumerical simulation \\
\hline LES & large oddy simulation \\
\hline LDA & laser doppler ancmometry \\
\hline FIT & Cast fourien transform \\
\hline$R \wedge N S$ & Reynolds averaged Navier Stokes \\
\hline $\mathrm{SF} / \mathrm{S}$ & stable focts/stretching \\
\hline $\mathrm{SN} / \mathrm{S} / \mathrm{S}$ & stable node/saddle/saddle \\
\hline $\mathrm{UF} / \mathrm{C}$ & unstable locus/contracting \\
\hline $\mathrm{UN} / \mathrm{S} / \mathrm{S}$ & unstable node/saddle/saddle \\
\hline
\end{tabular}




\section{Chapter 1}

\section{Literature review}

The sphere is arguably the most basic of three-dimensional geometries and serves as an excellent prototype for understanding the wake flow of more general bluff bodies, where separation is not fixed by the geometry. The study of sphere flows dates back to the days of Sir Isaac. Newton, who is attributed with the first measurements of sphere drag. Since then the problem has received ongoing attention due to its many enginecring applications. These include combustion processes, particulate transport processess and atmospheric flows, where the prediction of particle velocitics and trajectories is of importance. In gencral, particles in these environments are subject to both translational and rotational velocities as a result of particle particle and particle wall collisions and the ambient shear. For example, in many atmospherie flows, a sufficiontly strong wind blowing over a bed of particles will tend to pick particles up and (ause them to spin and skip along the bed as a result of surface roughness and the boundary layer shear. These particles will in turn collide with other particles and cause these to be ejected into the flow stream. This process is known as saltation and is responsible for the unique topology of desert, regions.

In order to simulate particulate flows, Lagrangian simulation mothods, which track the trajectories of a large number of particles, are often used. Here, it is necessary to be able to predict the forec and torque to which the particles are expesed. Data is still lacking for this purpose. This point is cmphasized by Pan et al. (2001) who are forced to assume a crude model for the lift force acting on particles in a rotating 
channel flow simulation. They state that "the lift force due to rotation of the particle for Reynolds numbers greater than 1.0 is still an open question." Understanding the flow past a spinning sphere, which is the focus of this study, is just onc important step towards understanding the flow past particles in more complex cnvironments. $\dagger$

In particle-laden flows, the particles may not bchave simply as passive scalars, but may work to actively modify the surrounding fluid flow. In some cases, vortex shedling from particles may have a significant effect on the cnhancement of turbulence, so that. an understanding of the sphere wake structure is of interest. Although several studies have considered the problem of a spherical particle in frec translation and rotation (sce, for example, Bagchi \& Balachandar (2002a)), the complexity of this problem makes it difficult to establish the physical process that shapes the wake, particularly when the more controlled problem is not yet understood. In an effort to acquire a fundamental understanding of the wake structure of a rotating sphere, this study considered two related problems. First, the wake structure of a spherical particlo in steady rotation about an axis transverse to the frecstream flow was investigated in the Reynolds number range 100 to 300. Although a number of studies have investigater this problem, their cmphasis hats been on the forces experieneed by the sphere. Second, the effect of unsteady particle rotation was investigated by simulating the flow past a sphere mondergoing oscillatory rotalion at a frecstream Reynolds number of 300 .

In this chapter a critical revicw of the relevant, literature is presented. Despite the munltitude of studies dealing with the flow past a sphere, many fundamental cunestions remain mnanswered. Section 1.1 begins by describing the uniform flow past a stationary sphere as a necessary reference for understanding the case when sphere rotation is introduced. Section 1.2 reviews the significant studies that have dealt with the uniform flow past a sphere in stcady rotation. The findings are introduced in increasing Reynolds number order; and the scope of the review is greater than that of the sub-

$\dagger$ In addition to providling the understanding necessary to solve practical problems, the flow past a sphere is inherently interesting. This appears to have motivated a number of studies, where the introductory justification for the work reads as an afterthought. 
sequent investigation so as to place the present study into the context of the broader problem. Section 1.3 discusses rotary oscillation. To the authors knowledge, the flow past a sphere in sinusoidal rotary oscillation has not becn studied previously, so studies that have considered a rotationally oscillating circular cylinder are discussed. Finally, the aims and outline of the thesis are presented.

\subsection{The uniform flow past a stationary sphere}

Our first exposure to the problem of the uniform flow past a sphere is generally the drag coefficient versus Reynolds number curve illustrated in figure 1.1. At moderate Reynolds numbers, this relatively smooth curve masks a series of fascinating flow bifurcations that lead to increasing levels of flow complexily. These various flow regimes are described in this section.

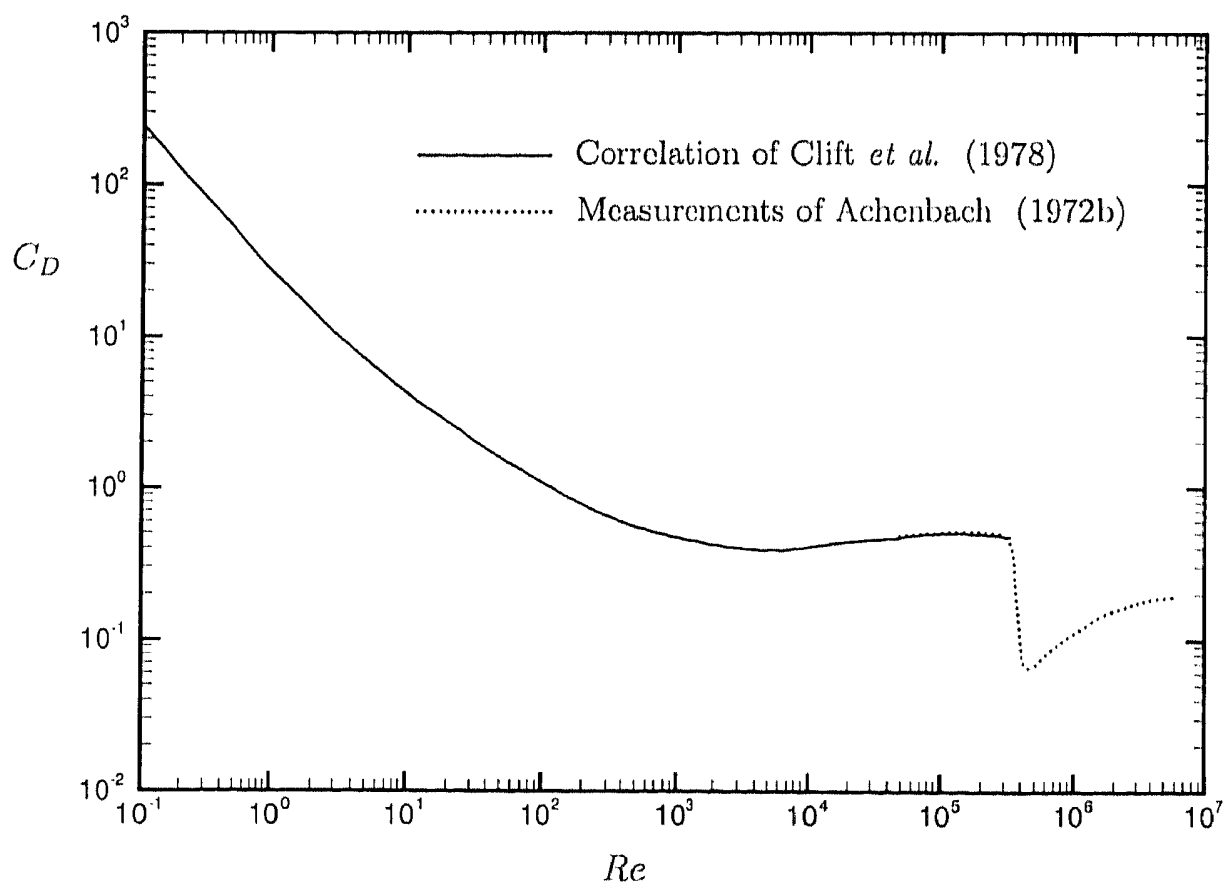

Figure 1.1: Drag cocfficient versus Reynolds number for a smooth sphere. The piecewise correlation derived by Clift is presented for $0.1 \leq R e \leq 3.38 \times 10^{5}$. The experimental measurements of Achenbach are around the critical regime and cover the range $5 \times 10^{4} \leq R e \leq 6 \times 10^{6}$. 
Using flow visualization, Taneda (1956) captured the wake pattern immediatcly downstrcam of a sphere for Reynolds numbers in the range $R e=9.15$ to 133 . Using a water tunnel towing tank facility and aluminium dust as the flow tracer, images of the near-wake streamline pattern were created by using a long time exposure. A subset of these photographic images are reproduced in figure 1.2. The patterns indicate that the flow first scparates from the rear stagnation point at a Reynolds number of about 25. In contrast, the flow visualization study of Nakamura (1976) suggested the existence of a recirculation eddy - cven at $R e=10$ or lower. Extrapolation of the numcrical results of Tomboulides \& Orszag (2000) and Shirayama (1992) indicates scparation to occur above a Reynolds number of about 20 , in support of the work of Taneda.

Above this transition Reynolds number, figure 1.2 illustrates the formation of a closed recirculating wakc, which is axisymmetric, stcady and toroidal in structure. $\Lambda$ s the Reynolds number is increased in this axisymmotric regime, the wake length and wake separation angle grow. Despite the growth of the toroidal wake, the numerical simulations of Johnson \& Patel (1999) show that a pressure minimum is not secn in the core of the toroidal wake until $R e \approx 200$. At lower Reynolds numbers it appears that the centrifugal force ereated by the vortex rotation is balanced by viscous foreces rather than a radial pressure gradient.

Topologically, the flow remains self-similar until a regular bifurcation at $R e \approx 212$. The stability analysis of Natarajan \& Acrivos (1993) found this instability to occur at $R e=210$ and reported the most unstiable mode to be non-axisymmotric with awimuthal mode 1. Tomboulides \& Orszag (2000) monitored the growth rates of all the azimulhal morles and found mode 1 to become unstable above $R e \approx 212$. In gencral, experimentally derived values for the regular transition tend to be lower than those predicted numerically, presumably due to pertirbations introduced by model supportis and fresstream anomalics. Taneda (1956) observed a faint periodic pulsative motion with a very long period at the rear of the wake above $R e=130$. Nakamura (1976) observed a loss of axisymmetry above a Reynolds number of 190, while Ormiéres \& 

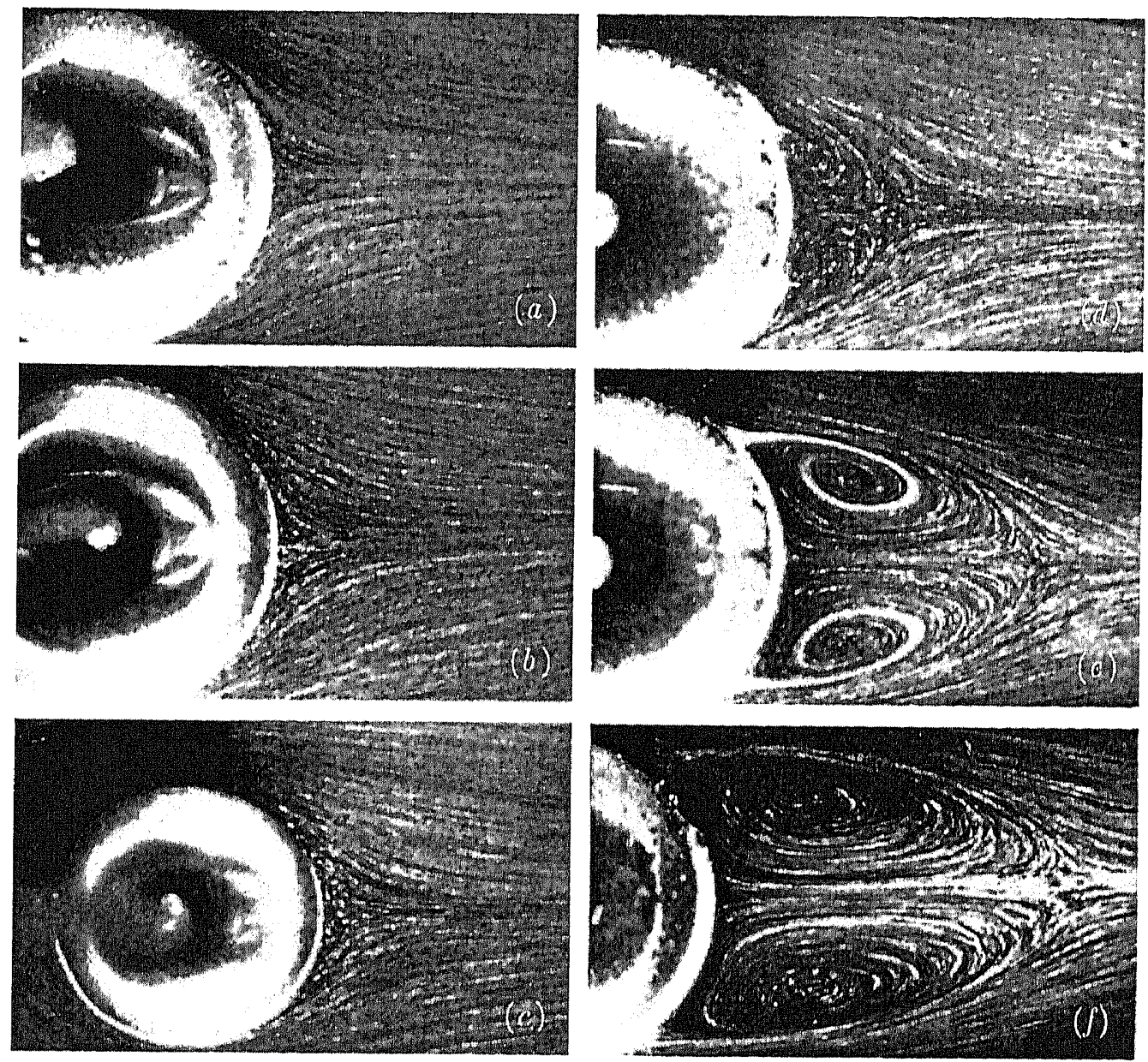

Figure 1.2: Flow visualization of the sphore wake using aluminium dust in water. Streamline patterns are shown for a range of Reynolds numbers in the steady axisymmotric: regime: $(a) R e=17.9 ;(b) R e=25.5 ;(c) R e=26.8 ;(d) R e=37.7$; (c) $R e=73.6 ;(f) R e=118$. Images taken from Tancda (1956).

Provansal (1999) observed two steady wake trails above $R e=180$. Magarvey \& Bishop) (1961b) and Magarvey \& Bishop (1961a) conducted flow visualization experiments on falling drops in an immiscible liquid and observed a transition from a 'single-thread" to a 'double-thread' wake structure at approximately $R e=210$, which is in excellent. agreement with the numerical studies mentioned earlier. Tomboulides (1993) showed that below $R e=270$ the flow reaches a steady-state solution, which, when perturbed, returns to that state after undergoing wave-like oscillations with a slow rate of decay. 
Tomboulides reasoned that the low amplitude wake oscillations, without distinct vortex shedding (which has been reported as the transition to unsteadiness in a number of experimental studies), may be due to ambient noisc. This noise triggers oscillations that appear to be self-sustained due to their low decay rate.

Above the regular transition Reynolds number, the wake remains stcady but the vortex ring becomes tilted off the streamwise axis. $\dagger$ Johnson \& Patel (1999) asssociated this transition with an azimuthal instability of the low-pressure core of the wake vortex. The shift in the vortex structure results in the azimuthal vorticity generated at the sphere surface having streamwise components that lead to the formation of two streamwise vortical tails of equal strength and opposite sign. Although axisymmetry is lost, the flowficld exhibits a symmetry plane passing between these vortical thread structures. The threads mutually interact as they travel downstream and are convected away from the streamwise centerline axis. The wake no longer forms a closed structure, and flud becomes entrained from upstream, spirals into the wake, and is released into the two streamwise tails. Magarvey \& Bishop (1961a) and Thompson el al. (20)(1) presented examples of the wake structure in the non-axisy mmetric steady regime using the flow vistalization of dye in water. The image presented by Thompson el al. is for $R e=270$ and is reproduced in figure $1.3(a)$. This depicts the recirculating region dividing into lwo filamonts. Although these streamwise vorticity threads are relatively woak in comparison to the azimnthal vorticity, they act as stable foci, thus contraining the dye to produce this striking inage of the double-thend structure. Figure $1.3(b)$ shews the wake strueture from a secend onthogronal view and highlights the wake asymmotry. Both inages ane from work by leweles and are also published in Sunts \& L Lim $(20)(0))$.

The wake asymmetry results in a lateral force directed in the plane of flow symmetry. This was observed in the experiments of Magarvey \& Bishop (1961a), where the path

$\dagger$ This is in contrast to the flow past a cylinder where the first transition is a Hopf bifurcation to a vortex sherding regine. 

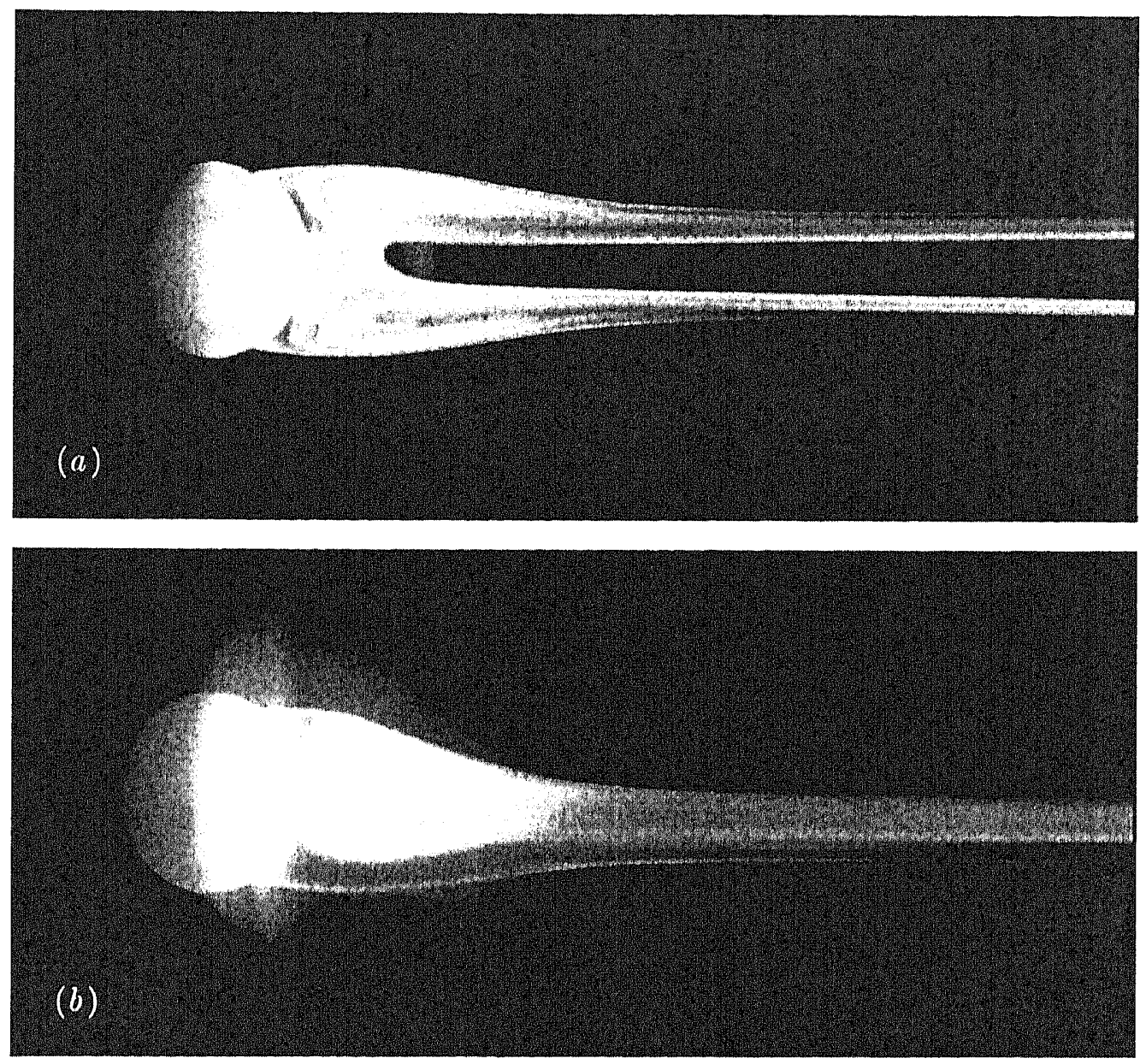

lighure 1.3: Flow visualization of the sphere wake structure at $R e=270$. The flow is makte visible using fluorescent dye in water. The pair of photographs show the flow from two perpendicular views. Images provided by Leweke.

of the licpuid sphere was secu to deviate from a vertical line of fall. Johnson \& Paldel (1999) (quantified these observations by presenting the lateral lift coeflicient against the Reynotds number. In laboratory experinents, the orientation of the symmetry p) lane is random, presumably determined by such things as noise in the annbient flow, model supports and other anomalies. Numerically, truncation error and grid topology may dietate the orientation of the symmetry plane. For computations using spectral based spatial discretization, the absence of significant truncation errors may require an 
artificial perturbation to trigger the flow asymmetry.

As the Reynolds number is increased in the axisymmetric regime, the streamwise vorticity tails strengthen and become elongated until the wake undergoes a Hopl biCurcation at a Reynolds number of about 280. The numerical simulations of Johnson $\&$ Patel (1999) predicted this transition to occur at $R e \approx 270$, in agrecment with Tombonlides \& Orszag (2000) who found the transition to occur between Re: =270) and $R e=285$. The stability analysis of Natarajan \& $\Lambda$ crivos (1993) found the transition to occur at Re $=277.5$ using an axisymmetric base flow for their antalysis. Expertimcentally Ormiéres \& Provansal (1999) found the transition to occur at $R e=280$ by monitoring the velocity fluctuations in the sphere wake. Above the Hop h hifurcalion the planar symmetry seen at, lower Reynolds numbers is maintained, while the flow is now characterized by the one-sided shedding of hairpin vortices at a single shedeling frequency. This flow structure has been captured using experimental flow visualization by Leweke et al. (1999) and Johnson \& Pated (1999), among others. In all casces, mly the one-sirded hairpin structures that originate at the surface were revealed. The result of Leweke al al. (1999) using dye in water for $R e=320$ is presented in ligure 1.4 .

Based on his own observations, Achenbach (1974) presented a schematic meresentation of the threcelimensional wake formation at $R C=1000$. The sketch is presented

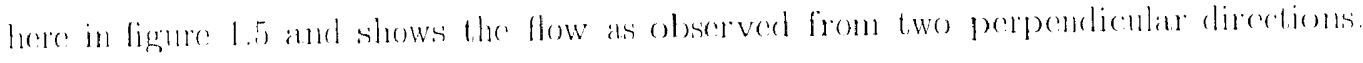

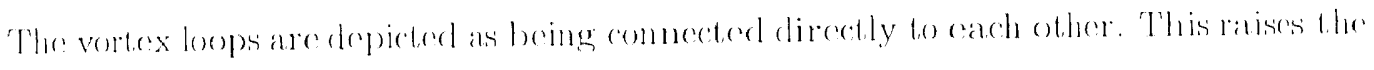

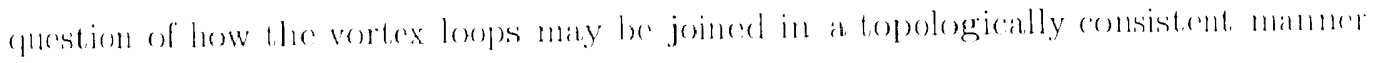
and whether the circulation in the wake conld be bounded if all the struetures have the same sign eirenlation. These concerns were clarified by Johnson \& Patel (1999)) nsing at mumerical simulation at $h e=300$. By applying the vortex identification method of Jeng \& Hustain (1995) they identilied, for the first time, previously mmeventorl haippin vortices of opposite sign and orientation to those shed from the sphere. The appoarance of these structures was described as being induced by the interaction of the near-wake flow and the outcre flow. A typical oblique view of the instantancous 

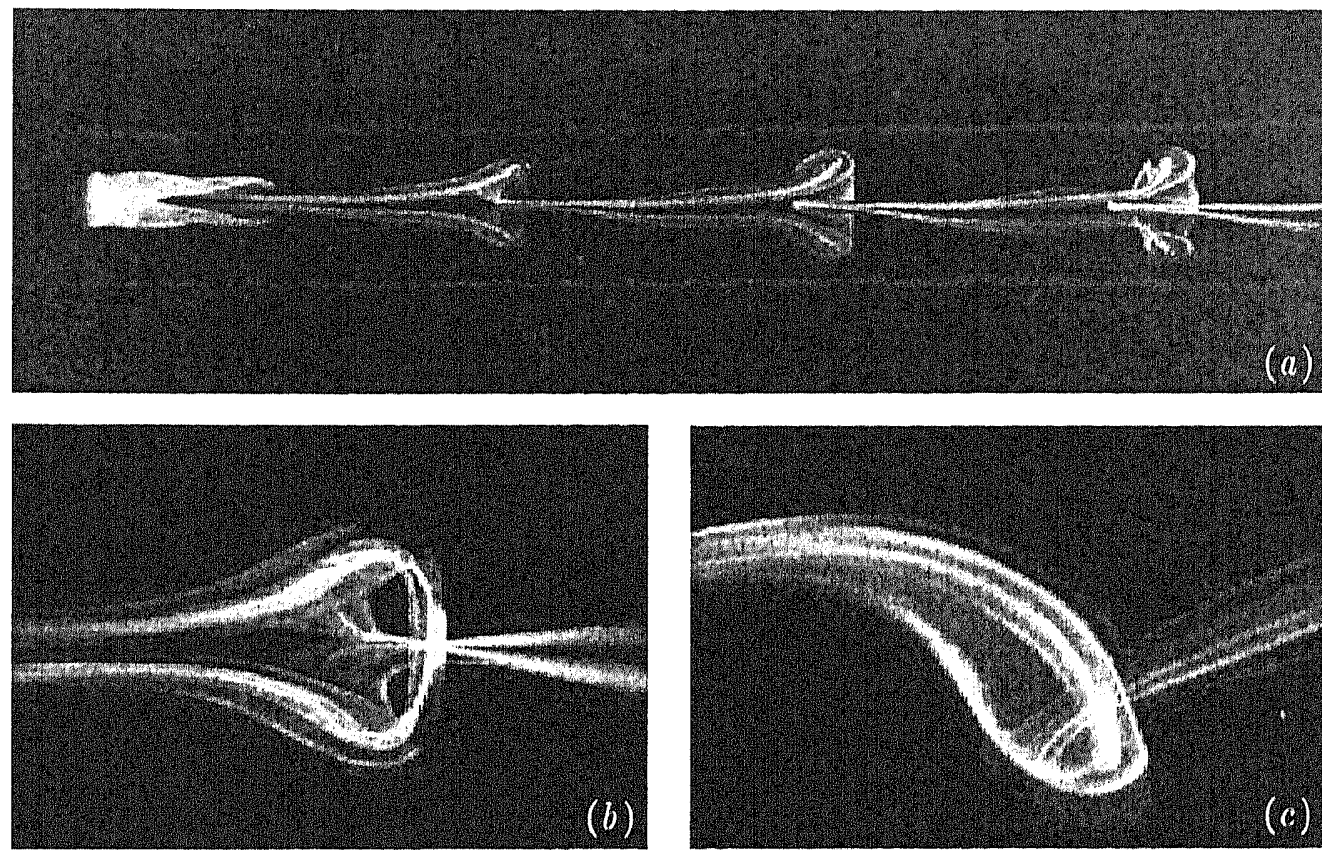

Fignte 1.4: Flow visualization of the sphere wake at he $=320$ using fluoresecnt dye in water. (a) shows a plane of symmetry through the wake and the periodic shedding of vortex loops. (b) and $(c)$ show close ups of a vortex loop from two perpendicular. views. Images taken from Leweke el al. (1999).

vortical structure for $R e=300$ is shown in figure 1.6 , where the hairpins shed from 1.he sphere surface are labelled $S_{1}$ and the induced hairpins are labelled $S_{2}$. With the primary hairpins connected to induced hairpins, rather than directly to each other, the t.opological inconsistencies cvident in the sketch of Achenbateh (1974) are avoided.

As the Hopl bifurcation Reynolds mumber is approached, Thompson et al. (2001) showed that the trailing streanwise structures exhibit an increased kinking at al)ont there radii downstream from the sphere center, which they suggested is the precursor 1.) the onset of vortex shedding. Schouveiler \& Provansal (2002) showed that the l.wo streamwise tails oscillate symmetrically close to and above the transition Reynolds mumber. The amplitude of the oscillations inereased until the vortical threads meet and reconnect to form loops, which are subsequently advected downstream. This instability is similar to that described by Crow (1970) for a pair of stationary comnter-rotating 

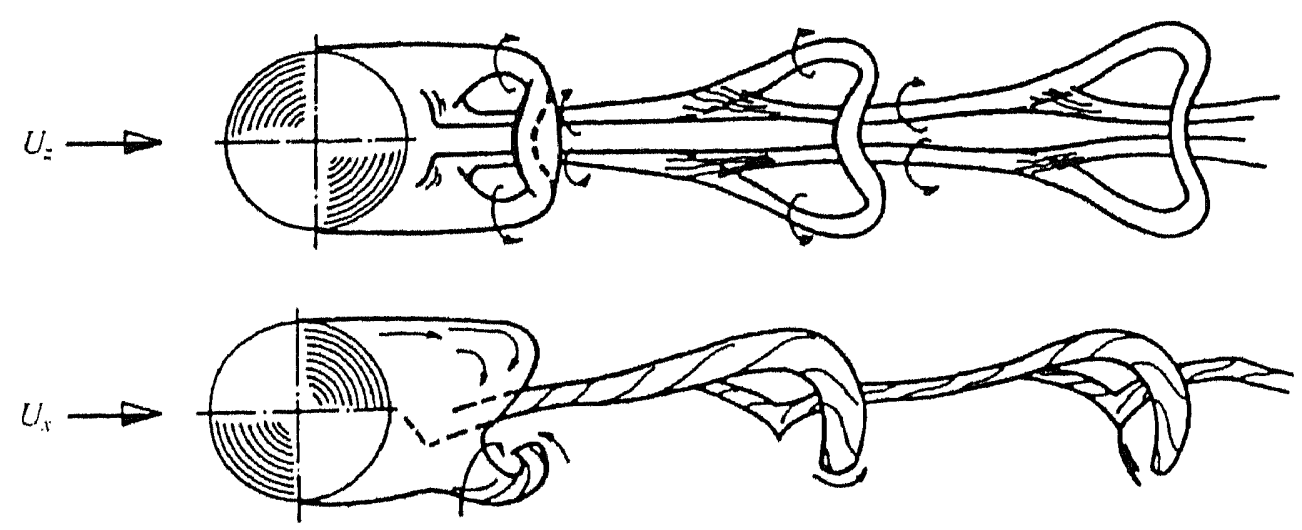

Figure 1.5: Vortex configuration in the wake of a sphere at $R e=1000$ as depicted by Achenbach (1974).

vortices, which undergo symmetric and sinusoidal oscillations until they eventually join at intervals to form a scrics of vortex rings. $\dagger$

Magarvey \& Bishop (1961b) described the shedding process as a simple build-up and release of a portion of the vortex ring in which the vorticity is greatest. Johnson \& Patel (1999) proposed a congruent physical mechanism driving the unsteady vortex shedding process. In the asymmetric steady regime, an azimuthal pressure gradient is established along the core of the toroidal wake vortex. This pressure gradient drives flow azimuthally around the wake such that the rate at which fluid is entrained into the wake from the upstroam flow is balaneed by the rate at, which fluid is cjected into the streamwise tails. Above the transition Reynolds number this equilibrium is lost and Iluicl entrainment is higher than the rate at which it can be ejected. Subsequently, the side of the toroidal wake which entrains fluid, begins to grow until the pressure field is such that this side of the wake vortex is expelled into the near-wake and is convected downstream. This process of growth, release and subsequent regeneration occurs periodically. The rate at which the process occurs dictates the shedding frequency of the wake, which is commonly non-dimensionalized by the frecstream vclocity and the

$\dagger$ The Crow instability is often seen in the wake of full-scale aircraft. Here, the low pressure in the cores of the wing-tip vortices lead to condensation and render them visible. 
sphere diameter to form the Strouhal number, $S t_{n}$. The vortex shedding frequency computed from various numorical simulations at $R e=300$ are in excellent agrecment, with values of $S t_{n}=0.137,0.136$ and 0.136 having been reported by Johnson \& Patel (1999), Tomboulides (1993) and Constantinescu \& Squires (2000), respectivcly. Experimentally derived values are gencrally higher, with a value of $S t_{n} \approx 0.15$ reported by Sakamoto \& Haniu (1990). As a result of the one-sided vortex shedding, the drag and lift forees both oscillate at the shedding frequency, in contrast to the eylinder flow case where the drag oscillates at twice the shedding frequency. The one-sided shedding also leads to a net lift force that acts along the plane of symmetry. This is also in contrast to the cylinder where the average lift is zero.

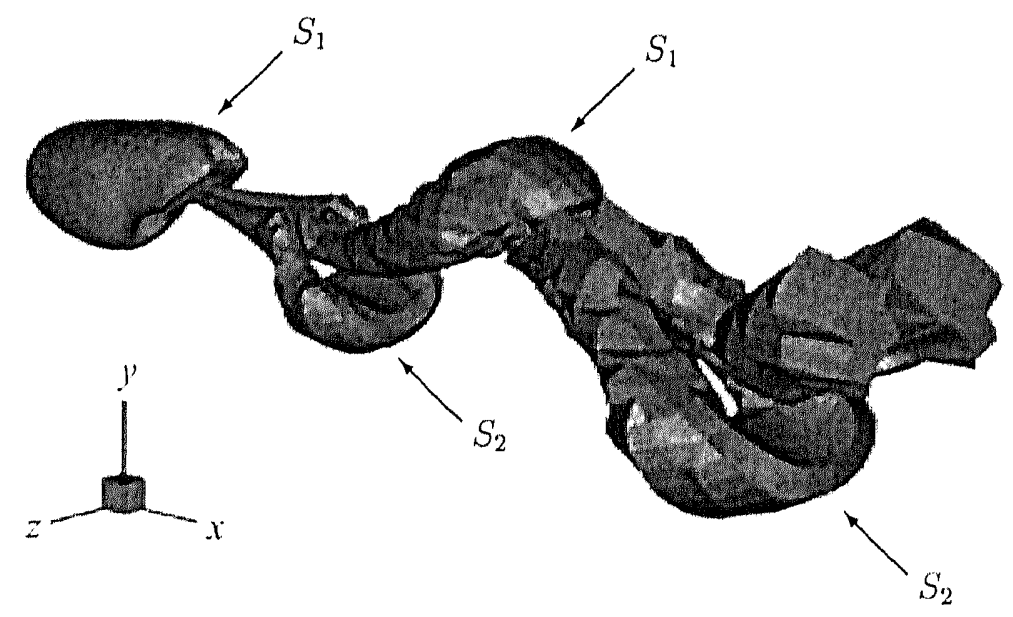

Figure 1.6: Instantancous vortical structure computed by Johnson \& Palcl (1999) for $R e=300$.

As the Reynolds number is increased in the vortex shedding regime, planar symmetry is maintained until a further bifurcation occurs beyond which cycle-to-cycle variations in the azimuthal orientation of the vortex loops formed in the near-wake are observed. The experimental flow visualization of Sakamoto \& Haniu (1990) observed the onset of this loss of planar symmetry at $R e \approx 420$, whereas the numerical simulations of Mittal (1999a) indicated planar symmetry to be lost in the Reynolds number range 350 to 375 . Mittal attributed this discrepancy to the inability of the experi- 
mental flow visualization to detect small-scale variations in the azimuthal angle of the vortex formation. Spectral analysis of the wake velocity measurements of Ormićres \& Provansal (1999) indicated a low frequency component above a Reynolds number of 360. This frequency appears to be associated with the variation in the azimuthal angle of the shed vortex loops, confirming the finding of Mittal (1999a). Mittal et al. (2002) showed that the loss of planar symmetry docs not immediately lead to a statistically symmetric wake, with the cycle-to-cycle variations in the azimuthal direction having a preferred orientation. For Re $=500$, Tomboulides (1993) and Mittal \& Najjar (1999) found a low frequency component with $S t_{n} \approx 0.040$ and $S t_{n}=0.045$, respectively, in addition to the dominant vortex shedding frequency. Tomboulides (1993) associaterl this low frequency component with the irregular rotation of the separation point azimuthally around the rear of the sphere, which comes with the loss of planar symmetry. From the onset of vortex shedding at $R e \approx 280$ up to Re $\approx 800$, the vortex shedding Strouhal number increases steadily to $S t_{n} \approx 0.2$.

Above Re $\approx 800$ the vortex sheet separating from the sphere undergoes a Kelvin Helmholtz instability, resulting in the appearance of shorter length scales in the wake. This causes the large-scale vortex loops to diffuse rapidly as they are convected downstream. The wind-tumnel experiments of Kim \& Durbin (1988) found two modes of unstcadiness when the Reynolds number execeded 800. Using a single hot-wire probe, al low frecuency could be deteded over a large region in the wake, while the higher frecuency was detected only in the regiom immediately downstream of the sphere. This lex Kin \& Durbin to rationalize that the high-mode was due to a Kelvin Holmholtz instability. Sakamoto \& Haniu (1990) and Bomneton \& Chomax (1992) also report,ed l.wo frequencies that bifurcate from a single frecquency state at a Reynolds number of about, 800). Sakamoto \& Haniu measured the vortex shedding Strouhal number in a wind-tumel using a hot-wire probe and visually estimated the shedding frequency using a separate water-tunnel flow visualization facility. Their measurements of the vortex shedding Strouhal number versus the Reynolds number are presented together 
with those of Kim \& Durbin (1988) in figure 1.7.

The water towing tank experiments of Bonneton \& Chomax (1992) used flow visualization to link hot-film velocity measurements of a high frequency mode with the appearance of smaller scales in the shear layer, thus confirming the hypothesis of Kim \& Durbin (1988). Kim \& Durbin found the Strouhal number of the high mode to vary as $R e^{0.75}$, whercas Bonneton \& Chomax (1992) showed their clata to collapse on a $R e^{0.5}$ law. The latter result indicates that the instability scales with the momentum thickness, which is consistent with a Kelvin Helmholtz instability. Bcyond the transition at $R e \approx 800$, the low frequency maintains a fairly constant value of $S^{\prime} t_{n} \approx 0.2$. At $R e=1000$, for example, Tomboulides (1993) reported a vortex shodeling frecpuency of $S t_{n}=0.195$, in agrecment with the experiments of Kim \& Durbin (1988) who report, $S t_{n} \approx 0.19$ and Sakamoto \& Haniu (1990) who report values in the range $S t_{n}=0.195$ to 0.205 .

There is less agreemont between datia collected at higher Roynolds numbers. The following elescription is based on the current state of knowledge and is by no means definitive. Above Re $\approx 3000$ the boundary layer is laminar on separation and transition to turbulence occurs in the free shear layer through a Kelvin Helmholtz instability. As the Reynolds number is increased further, the transition point moves upstream along the shear layer, such that by Re $\approx 6000$, the shear layer becomes fully turbulent on separating from the sphere. In the range $R e=3000$ to 6000 , Sakanoto \& Haniu $(1990)$ found a gradual drop in the Strouhal number for vortex shedding (see figure 1.7). They conjectured that the appearance of turbulence in the separated shear layer increases the dissipation in the near-wake, and as a result it lakes longer to store the cnergy necessary to drive the build-up and release cycle required for vortex shedeling. $\Lambda$ bove $R e \approx 6000$, the Strouhal number for vortex shedding gradually recovers to a fairly constant value of $S t_{n} \approx 0.2$.

Using smoke flow visualization, Taneda (1978b) showed that for Reynolds numbers between $1.0 \times 10^{4}$ and $3.8 \times 10^{5}$ the wake structure remains almost unchanged. The 


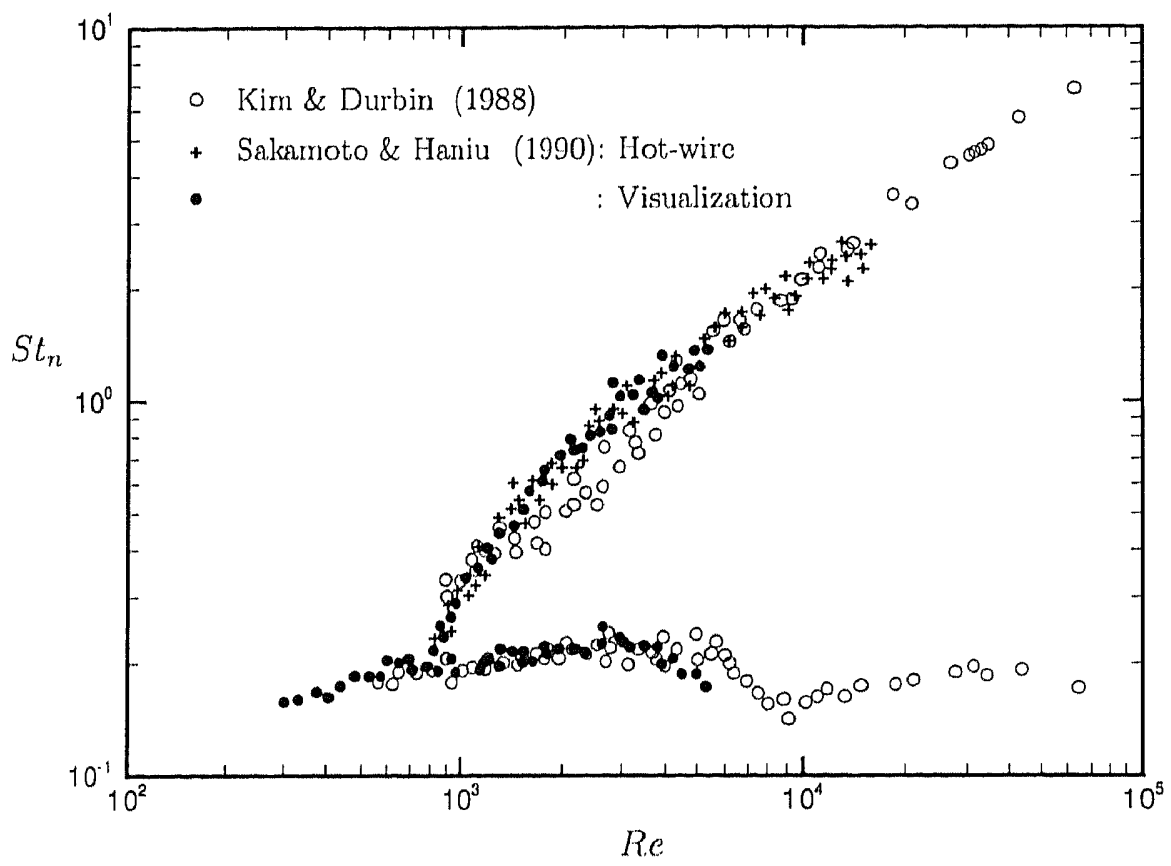

Figure 1.7: Experimental measurements of Strouhal number, $S t_{n}$, versus Reynolds number, Re.

wake undergoes a wave motion in a plane that inchedes the streamwise axis with a wavelength corresponding to $S t_{n} \approx 0.2$. The plane in which the oscillations occur rotates slowly and irregularly aboul, a streamwise axis through the center of the sphere. These findings correlate with the observation of Achenbach (1974) which suggest that, the vortex separation point periodically rotates around the sphere.

Constantinescu \& Squires (2000) applied Large Eddy Simulation (LES) for Re = $1 \times 10^{4}$. They use the dynamic procedure of German et al. (1991) and a Smagorinsky base modol to predict an moan drag cocficient of 0.393 , which is in reasonable agreoment with the value of 0.410 oblained from the experimentally derived correlation of Clift 
et al. (1978). $\dagger$ They found a large-scale shedding frequency of $S t_{n}=0.195$, while a shedding frequency associated with the Kelvin Helmholtz instability was not reported. Tomboulides (1993) performed LES at Re $=2 \times 10^{4}$ using a subgrid scale model based on renormalization group theory. He reported a mean drag coefficient of approximately 0.43 (estimated from figure 19 of his dissertation), while the correlation of Clift el al. (1978) predicts a value of 0.44 . The large-scale shedding frequency was found to be in the range $S t_{n}=0.18$ to 0.19 . Close to the separation point, the shear layer instability was detected with a shedding frequency of $S t_{n} \approx 3.5$ which is in close agreement with the hot-wire measurements of Kim \& Durbin (1988), who found a value somewhere in the range of 3.2 to 3.8 (estimated from figure 2 (b) of their paper). The instantaneous wake structure is in qualitative agrecment with the flow visualization of Taneda (1978b) for $R e=2.3 \times 10^{4}$.

For Re $=1 \times 10^{4}$, Constantinescu \& Squires (2000) found a laminar separation oceurring between 84 and 86 degrees from the upstream pole of the sphere. At Re $=2 \times 10^{4}$ Tomboulides (1993) found separation to occur at. 88 degrees from the upstream pole of the sphere. Taneda. (1978b) also measured the angle of flow separation using surface-oil flow visualizations in the range $10^{4}<R e<10^{6}$. In the range $10^{4}<R e<3.5 \times 10^{5}$ the separation angle was found to remain almost unaltered at aboul 80 degrees from the upstream pole. Achenbach (1972b) inferred the separation from the surface local skin friction and the surfece pressure. For $R e=1.62 \times 10^{5}$ the boundary layer was found to undergo a laminar separation at approximately 82 degrecs. To late, Direct Numerical Simulation (DNS) or LES above Re $=2 \times 10^{4}$ have not, been reported in the open literature. The Reynolds Averaged Navier Stokes equations have been solved at higher Reynolds numbers, but in general these do not adequately model the wake physics and therefore do not warrant discussion in the present context.

$\dagger$ Brown \& Lawler (2003) combined the data of a number of studies for Reynolds mumbers up to $2 \times 10^{5}$ and reject suspect data points. They then evaluated a number of existing correlations aganst, this new data set. By evaluating the sum of the squares of the relative errors, they conchded that the correlation of Clift et al. (1978) modelled the sphere drag versus Reynolds number relationship best. 
Tancda (1978b) showed that above $R e \approx 3.8 \times 10^{5}$ the periodic large-scale wakc fluctuations cease suddenly and the near-wake region shrinks. This reduction in the wake size results in a sudden reduction in the drag cocfficient (as illustrated in figure 1.1) and the corresponding Reynolds number at which the minimum occurs is often referred to as the 'critical Reynolds number'. The vortex shect separating from the sphere becomes offset from the streamwise axis and rolls up into a vortex pair. These strcamwisc vortex tails mutually interact as they move downstream and are convected away from the streamwise axis. The surface-oil flow patterns at the same Reynolds number indicated laminar separation at about 100 degrees from the upstream pole, followed by a reattachment at about 117 degrees and a subsequent turbulent separation at about 135 degrees. These results are in reasonable agrement with the findings of Achonbach (1972b).

Using the hot-wire measuring technique, Achenbach (1972b) measured a minimum drag coefficiont at $R e \approx 3.7 \times 10^{5}$ and could not detected vortex shedding beyonel this Reynolds number. Local skin friction distributions showed a laminar separation followed by turbulent reattachment, between $R e=3.18 \times 10^{5}$ and $R e=1.14 \times 10^{6}$. As the Reynolds number was increased in this range, the laminar separation bubble becano progressively smaller, and at higher Reynolds numbers the transition from laminar to turbulent flow occurred without an intermediate laminar separation. In this critical regime, the boundary layer is particularly sensitive to frecstream turbulence and surface roughness, which may aceount for differenees in the critical Reynolds number reported in various studics. For example, with increased surface roughness Achenbach (1972a) fonnd that transition to turbulence is promoted, leading to a decrease in the critical Reynolels number, while beyond the critical value a higher drag coefficient results. As the Reynolds number is increased beyond $R e \approx 1.5 \times 10^{6}$, the transition from laminar to turbulent, boundary layer is shifted upstream. 


\subsection{The flow past a spinning sphere}

The uniform flow past a sphere rotating at constant angular velocity, has been studied for two principle directions of rotation: about axes orthogonal to and parallel to the frecstream flow vector. We refer to these two scenarios as transverse and streamwise rotation, respectively. In relation to particle transport processes Torobin \& Ganvin (1960), among others, have noted that streamwise rotation is a far less common occurrence than transverse rotation. In the presence of a convection volocity, particle collisions with solid boundaries, collisions between particles moving at different convection velocitics and streamwise velocity gradients (which are dominant near boundaries), are most likely to induce rotation about an axis perpendicular to the flow direction. This review is fecussed on the case of transverse rotalion. To aicl discussion, the sphere is divided into two hemispheres as illustrated in figure 1.8. The hemisphere over which the surface velocity has a component in the same direction as the frecstream flow is referred to as the rotreating side (colored light grey); the hemisplere over which the surface velocity has a component, in the opposite direction to the froestrean flow is reforred to as the advancing side (colored dark grey).

The flow past a rotating cylinder is also discussed. This problem has been the sulfject of more intense research than the spinning sphere. This may, in part, be due to the two-dimensional nature of the flowfield at low and moderate Reynolds numbers, and the relative simplicity with which experiments can be conducted withont, significant. stupport, interference.

The uniform flow past a transversely rotating sphere is best, known for a transverse acrodynamic: forec, which leads to the deffection of its flight path. As carly as the scventecnth century, Newton (1672) noted that the flight path of a tennis ball was affected by spin. Later, Benjamin Robins, the British mathematician and military engineer, observed that a projectile spinning about its axis experiences a transverse force that causes it to deviate from a straight line of travel. Robins fired musket-balls 


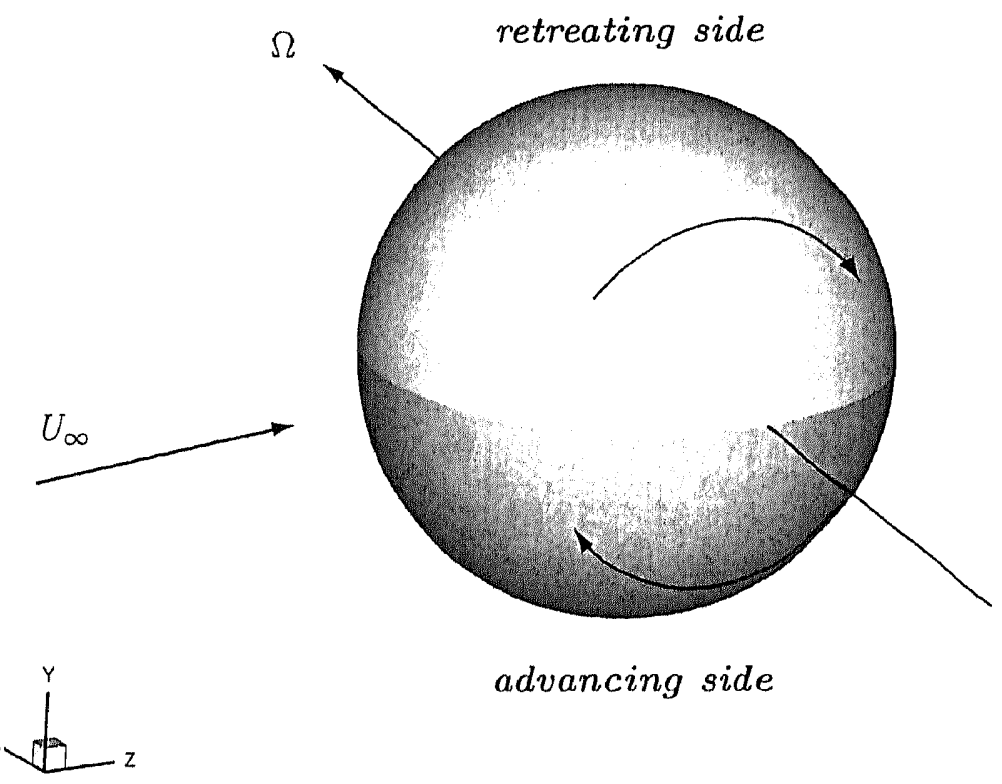

Figure 1.8: Definition of the edvancing and retreating sides of a rotating sphere.

from bent barrels, which forced the balls into conteact with the bore, causing them to have both translational and rotational velocity. By tracing the trajectories of the musket-balls on a series of thin paper screens, he established strong evidence of flight. path curvature with spin. Leonhard Euler, the dominant, hydrodynamicist of the time, rejected the possibility of a lateral force resulting from spin and attributed the result lo manufacturing imperfections of the projectiles. The finding was not aceepted as a real acrodynamic phenomena until much later when Gustav Magnus demonstrated that a spinning cylinder exposed to an airlow moves laterally. His exporiments were unsuceessful with spheres, but he infered that the effect was similar for the splere. Latcer Rayleigh (1877) attributed the first true explanation of the lateral deflection of a spinning ball to Magnus, from which the effect draws its name. Over a century later it was argued by some that. Robins' work did not receive the recognition that, it deserved. Barkla \& Auchterlonie (1971) suggested that "it would only be justice if 
the case of a sphere were to be renamed the Robins effect." Hore we follow the lead of Sengupta \& Talla (2004) and refer to it as the 'Robins Magnus effect'.

Rubinow \& Keller (1961), by means of matched asymptotic expansions, derived an expression for the forces acting on the sphere for Reynolds numbers less than unity. They found the drag foree to be independent of rotation and equal to the Stokes drag. $\Lambda$ lift force, $L$, orthogonal to the direction of motion was derived with the form $L=\pi \rho \Omega \times V$, where $\boldsymbol{V}$ is the freestroam velocity vector, $\Omega$ is the angular velocity vector and $\rho$ is the fluid density. When the angular velocity vector is orthogonal to the fresstream vector, the resultant lift force is maximum and can be writton in coefficient form as

$$
C_{L}=2 \Omega^{*}
$$

where $\Omega \Omega^{*}=\Omega\left(d / 2 U_{\infty}\right.$ is the non-dimensional rotation rate.

At moderate Reynolds numbers, the problem is only tractable via exporiment, or numerical simulation. Early experimental investigations focused on relating the Reynolds mumber and rotation rate to the forecs experienced by the sphere. At high Reynoleds mumbers, the splhere may be monnted in a wind-tumel via a spindle driven by an clectrice motor momuted on a force balanee. Tsuji el al. (1985) cmphasized that this method becomes inaceurate for Reynolds numbers lower than $10^{4}$ because the latceral fores on the sphere is toos small to be moasured accurately and the sphere becomes small in relation to the support mochanism. Instcad, they adopted an indirect method of measurement for lie $=550$ to 1600 and $\Omega^{*}<0.7$. Spheres were made to bounce on an inclined flat, plate and the subsecuent range of flight, and the rotation rate of the sphere was measured from photographic images. The lift force acting on the sphere was recovered using the equation of momentum conservation. Here, the drag coefficicnt wats assumed to be independent of rotation and was estimated using the empirical equation given by Morsi \& Alexander (1972). By assuming proportionality between the lift coeflicient and rotation rate (as was found theoretically by Rubinow \& Keller 
for Reynolds numbers less than unity) the following expression was derived

$$
C_{L}=(0.4 \pm 0.1) \Omega^{*}
$$

In light of more recent studies at moderate Reynolds numbers, the assumptions of drag being independent of $\Omega^{*}$ and lift being linearly dependent on $\Omega^{*}$ are questionable. Despite this, the study does show that the lift force experienced by a transversely rotating sphere at moderate Reynolds numbers is less than that predicted at Reynolds numbers below unity.

Ocsterlé \& Dinh (1998) also used a trajectory technique to obtain the lift, force acting on a transversely spinning sphere for $R e=10$ to 140 and $\Omega^{*}=1$ to 6 . In this case, the sphere was fitted with a cylindrical axle that supported the sphere against gravity by two suspension throads. The threads, which were initially coiled about the axle, were traversed vertically leading to the transverse and rotational motion of the sphere. It was found that increasing $\Omega^{*}$ led to an increase in the lift coefficient, while beyond a Reynolds number of about 100 the effect of $\Omega^{*}$ vanished. Although the trajectory technique avoirls the inaccuracies of direct moasurement techniques, it, introduces a host of other crror sourecs and the measured values for the lift coefliciont were estimated to be aceurate to only 20 perent. A best fit to the data gave the correlation

$$
C_{L} \approx 0.45+\left(2 \Omega 2^{*}-0.45\right) \operatorname{cxp}\left(-0.075\left(\Omega^{*}\right)^{0.4} R e^{0.7}\right)
$$

The relationship between $C_{L}$, Re and $\Omega^{*}$ conld not be explained and it was noted that information concerning the flow structure around the sphere is required for this purpose. In figure 1.9 this correlation is presented for a range of representative Reynolds numbers. For comparison, the results of Rubinow \& Keller (1961), Tsuji el al. (1985), Ocsterlé \& Dinh (1998) and a number of other studies (yct to be introduced) are also shown.

The sources of error that, hinder these experimental studies are absent in numerical simulation, although a number of numerical issues arise. You et al. (2003) computed 

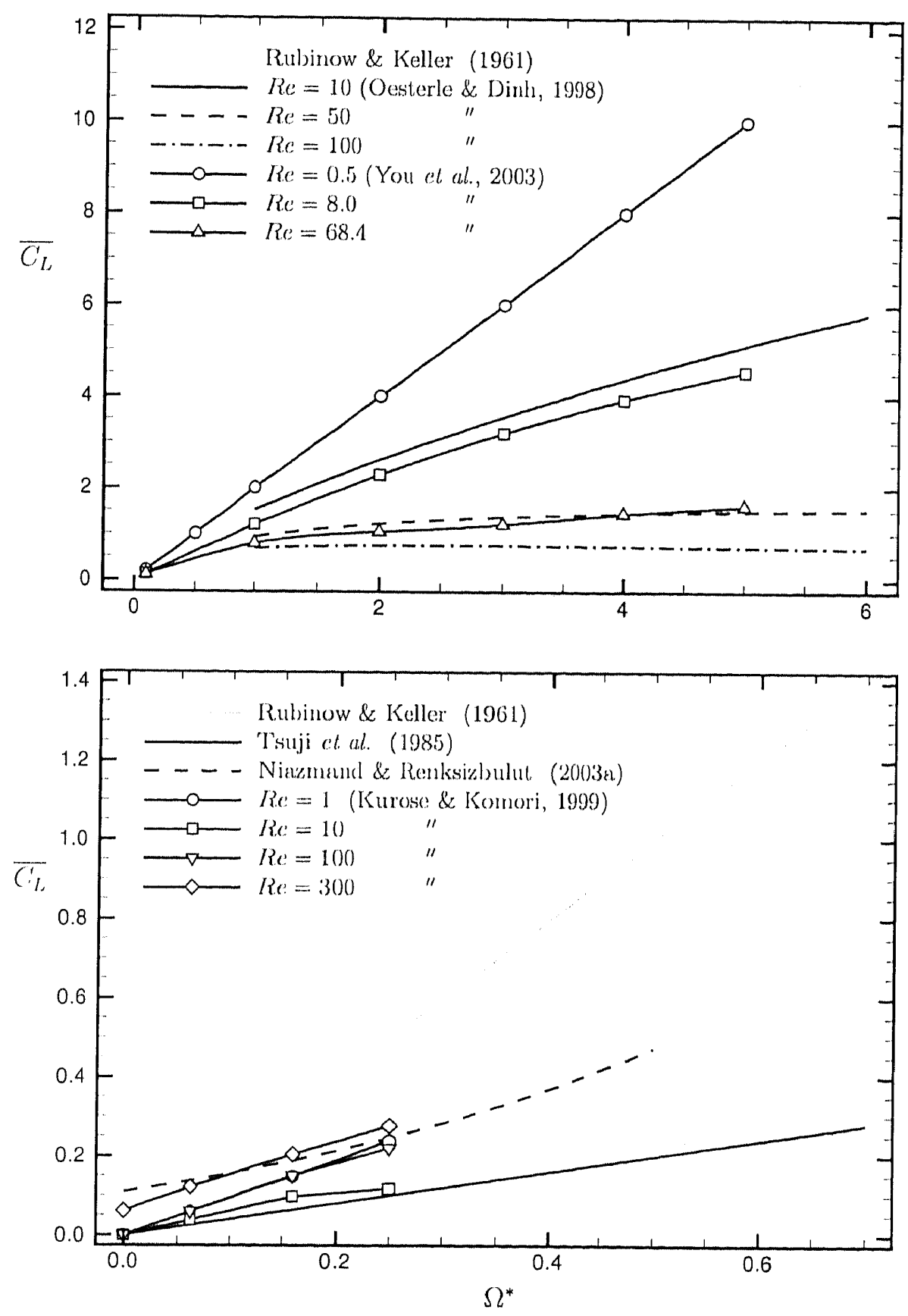

Figure 1.9: Timc-averaged lift coefficient, $\overline{C_{L}}$, versus $\Omega^{*}$. 


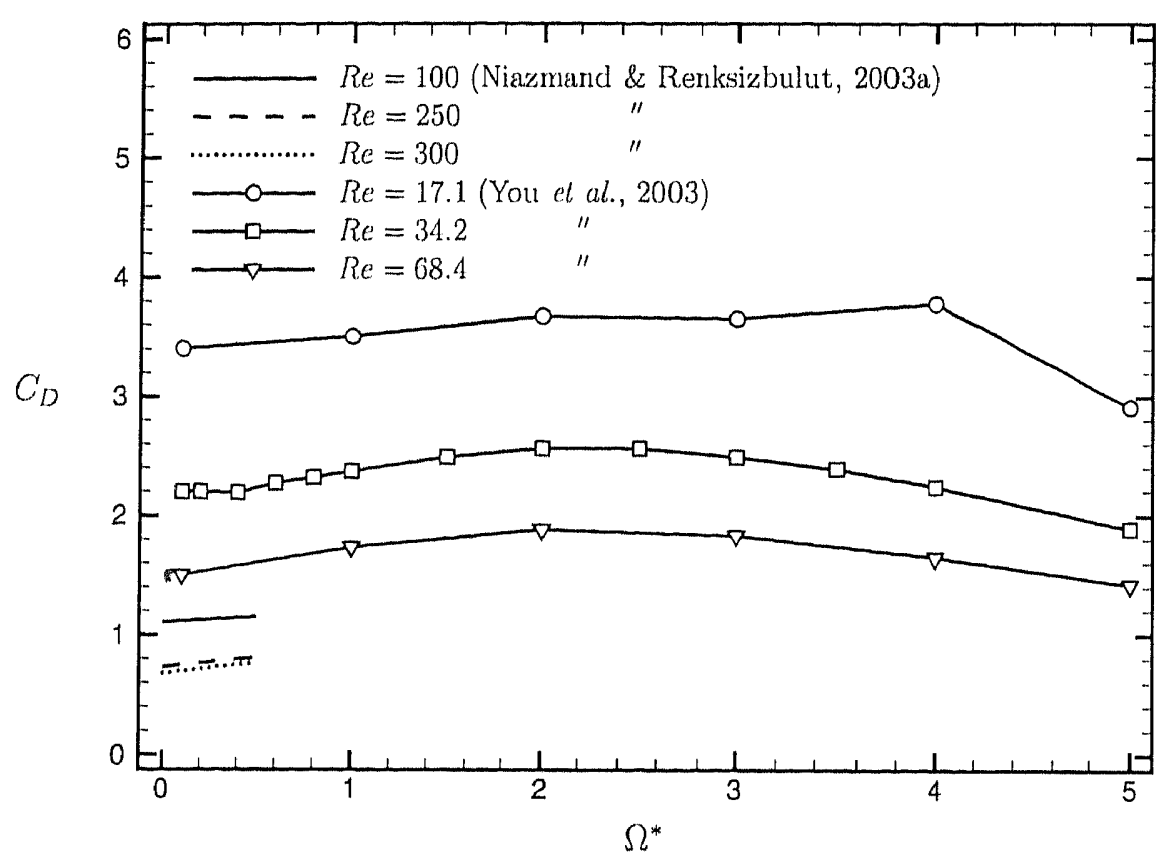

Figure 1.10: Timc-avcraged drag coefficient, $\overline{C_{D}}$, versus $\Omega^{*}$.

the flowfield for Re $=0.5$ to 68.4 and $\Omega^{*}=0$ to 5 using DNS based on a finite volume: spatial discretization. $\Lambda$ subsed of their lift and drag coefficient data is plotted versus $\Omega^{*}$ in figure 1.9 and figure 1.10, respectivoly. At Reynolds numbers less than unity $(R e=0.5)$, the lift coefficient was found to be lincarly proportional to $\Omega^{*}$ and equal to the theoretical value reported by Rubinow \& Koller (1961). For a given rotation rate, increasing the Reynolels number resulted in a monotonic decroase in the lift coefficient, strch that it approached a constant value for $R e>100$. These findings are in qualitative agrecment, with the experimental study of Oesterle \& Dinh (1998). For $\Omega^{*}<2$ the drag coeflicient increased slightly, while at higher rotation rates it decreased. The: authors acknowledged some doubt, in the results for high rotational specds and it is not clear if the trend at high $\Omega^{*}$ is physical. Wake patterns computed at $R e=34.2$ showed that increasing the rotation rate gradually reduces the wake size until it is completely 
destroyed. The wake suppression occurs in the range $0.1<\Omega^{*}<1.0$; the sparse test matrix did not allow a more precise estimate for the transitional value of $\Omega^{*}$.

Salem \& Oesterle (1998) applied DNS to the problem of a spinning sphere in the presence of an ambient shear flow. They considered Re $<40$ and rotational rates in the range $-2 \leq \Omega 2^{*} \leq 2$. The rotating sphere was shown to entrain a laycr of rotating fluid adjacent to the body, leading to a single stagnation point on the advancing side of the sphere, which is displaced some distane from the surface. The presence of both shear and rotation does not allow other conclusions to be drawn regarding the sole effect of rotation $\dagger$

Kurose \& Komori (1999) conducted a similar numorical study of a rotaling sphere in the presence of a shear flow, but did consider the case of rotation only. For $1 \leq R e \leq 500$ and $0 \leq \Omega^{*} \leq 0.25$, they found that rotation acts to shift the point of separation downstream on the retreating side and upstroam on the advancing side. The surface pressure and viscous stress distributions wore such that both the pressirure and viscous fores contributed positively to the lift force, with the pressure component making the greater contribution. The lift coefficiont increased with rotation rate. However, for a given rotation rate, the lift, initially decreased with Reynolds number and then increased until it approached a constant value for $R e \geq 200$. This bohavior is inconsistent, with other studies which showed the lift coefficient to tend smoothly towarels an asymptotic value as the Reynolds number is increased. Their lift coefficient versus $\Omega^{*}$ data for a range of Reynolds numbers is included in figure 1.9. The drag coefficient, also increased with rotation rate. For a given rotation rale, however, the drag coefficient showed a similar trend to that for a stationary sphere, that is, there is a rapiel decrease in drag with Reynolds number. For situations where unsteady vortex shedeling prevails, the Strouhal number for vortex shedding was found to increase with increasing rotation ratc. The reason for this was not clarified. The numerical study

$\dagger$ Kurose \& Komori (1999) have shown that at moderate Reynolds numbers $(R e=200)$ the effect of rotation and shear cannot be independently treated, unlike the situation at Reynolds number less than unity where linear superposition of the contributions is possible. 
of Pregnalato et al. (2001) also found the lift to level out in the manner reported by Kurose \& Komori. By analyzing the vortical structures in the wake, they found that this coincides with the onset of periodic vortex shedding.

Niazmand \& Renksizbulut (2003a) applied a finite volume based numerical simulation to the problem of a transwersely rotating sphere for $10 \leq R e \leq 300$ and $\Omega^{*}=0$ to 1. For a Reynolds number well below the regular transition for a stationary sphere (Re $\approx 212$ ), rotation leads to an asymmetric wake, while symmetry is prescrved about, a plane normal to the rotation axis. With increasing rotation rate, the recirculation zone becomes progressively smaller and is displaced to the advancing side of the sphere. For sufficiently large rotation rates the recirculation region is completely climinated. For $R e=100$ the wakc was found to be suppressed for $\Omega^{*} \geq 0.5$.

At Reynolds numbers approaching the regular transition for a stationary sphere, rotation can trigger a transition to vortex shedding. For $R e=200$, time-periodic: vortex shedding was attained for $\Omega^{*} \geq 0.5$. For lower values of $\Omega^{*}$, vortex shedeling was secn only in the transient solution. At Reynolds numbers approaching the Hope bifurcation for a stationary sphere, lower values of rotation are sufficient to trigger the (ransition to vortex shedding. For example, at $R e=250$ a rotation rate of $\Omega^{*}=0.05$ resulted in sustained vortex shedding. Increasing $\Omega^{*}$ further resulted in an increase in the vortex shectling Strouhal number an effect alse found for $R e=300$, where the flow past a stationary sphere is characterized by vortex shedding.

At $R e=300$, the drag increase due to rotation is predominantly due to the pressure component, with little change evident in the viscous component of drag. Furthermore, the effect of $\Omega^{*}$ on the drag is less pronouned at the lower Reynolds numbers eonsielcred. This trend is consistent with the limiting case of $R e \rightarrow 0$ considered by Rubinow \& Koller (1961), where the drag is completely unaffected by rotation.

Over the range of Reynolds number and rotation rate tested the average lift and drag force coefficients increase with increasing rotation rate. Good agrement is found with the data presented by Kurose \& Komori (1999). For $0.16 \leq \Omega^{*} \leq 0.5$ and 
$100 \leq R e \leq 500$ the lift and drag coefficients agrec within 20 and 10 percent, respectively, with the expressions

$$
\begin{aligned}
& C_{L}=0.11\left(1+\Omega^{*}\right)^{3.6} \\
& C_{D}=\frac{24\left(1+R e^{2 / 3} / 6\right)}{R e}\left(1+\Omega^{*}\right)^{R c / 1000} .
\end{aligned}
$$

Here the corrclation was extended to Re $=500$ by using the data of Kurose \& Komori (1999) in the range $300<$ Re $\leq 500$. The corrolation for the lift cocfficient is shown in figure 1.9. The corrolation for the drag coefficient is presented for a range of Reynolels numbers in figure 1.10. Tsuji et al. (1985) assumed the drag to be unaffected by rotation for $R e=550$ to 1600 . In light of equation (1.5), this assumption appcars not, lo be strictly correct.

A much needed exporimental study of the wake flow came from Best (1998), who considered moderate Reynolds numbers in the range $R e=29$ to 290 and rotation rates in the range $\Omega^{*}=0.0$ to 1.5 . Using synthetic oil as the working medium, a suspension of finc particles was used for flow visualization and as seeding for velocity measurements using Laser Doppler Anemometry (LDA). The study considered the flowlich (nn the two-dinensional streanwise plane normal to the rotation axis. Unfortunately, this does not reveal the full threc-dimensional nature of the wake. For all Reynolds mumbers considered, the mean wake length became progressively smaller with rotation and coased to exist for $\Omega^{*}>0.5$. The streak-lines at $R e \approx 140$ and for a number of representative rotation rates are shown in figure 1.11. The transition is congruent, with the wake suppression reported in the numerical studies of You el al. (2003) for $R^{\prime}=34.2$ and by Niazmand \& Ronksizbulut (2003a) for $R e=100$. For $\Omega^{*}<0.5$, rotation promotes the transition to unsteady vortex shedding, with a shedding frecuency that is modulated by the rotation rate.

For $\Omega^{*}>0.5$, fluid passing over the retreating side is dragged over the sphere lee side until it meets fluid moving downstream over the advancing side of the sphere and is 'peeled off' the surface to form a shear layer. It is expected that wake generation 
and eddy shedding is absent for these highor rotation rates.
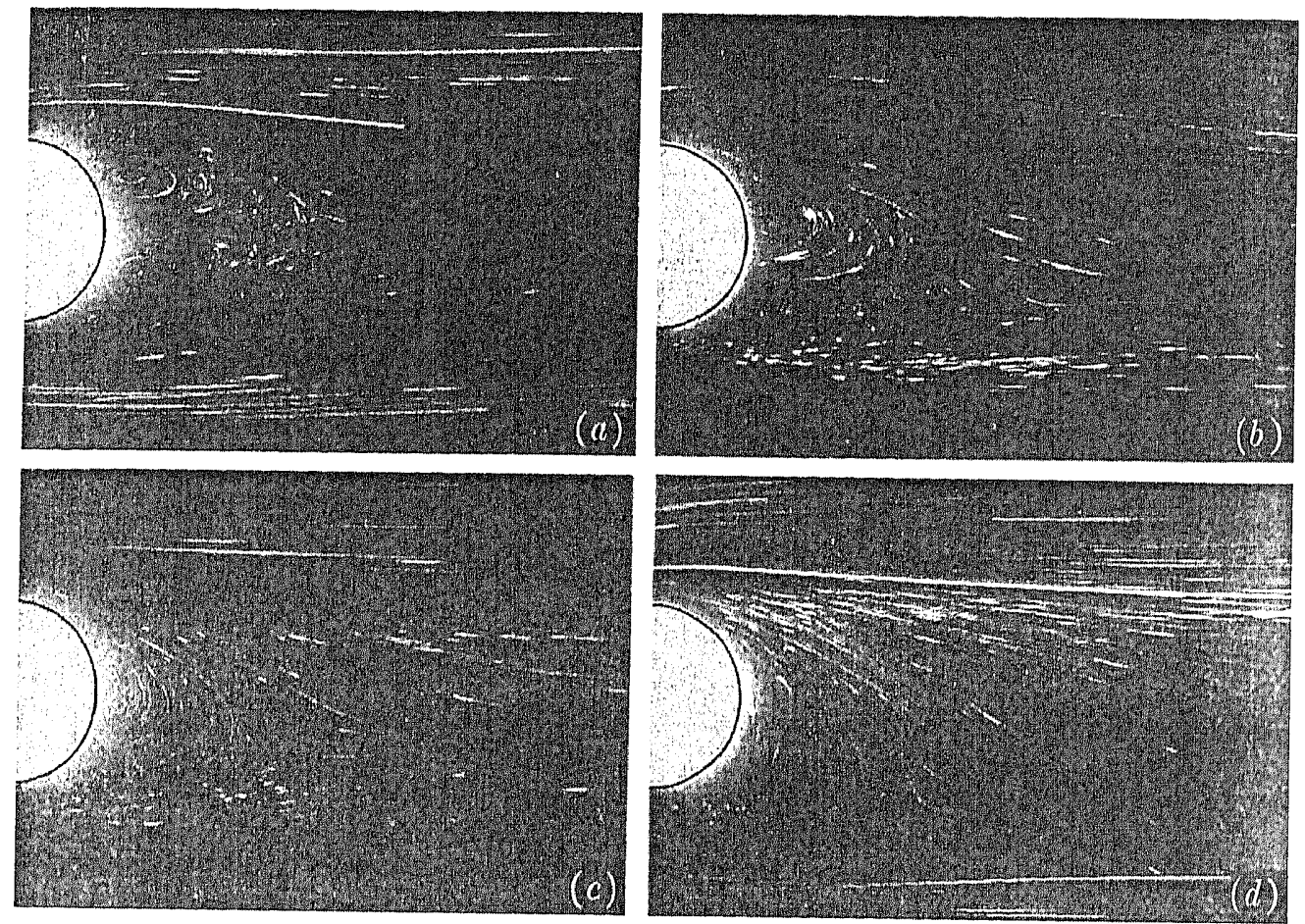

Figure 1.11: Sphere walke flow visualization at, $R e \approx 110$ for $(a) \Omega^{*}=0 ;(b) \Omega^{*}=0.15$; (c) $\Omega^{*}=0.30 ;(d) \Omega \Omega^{*}=1.50$. The working medium is oil and fine particles are used as the tracer. The particles appoar as streaks by using a long time exposure. Inage? tialken from Best. (1998).

Suppression of vortex shedeling has also been reported for the flow past a retating cylinder, althongh at significantly higher rotation mates. Using two-dimensional nutmorical simnlation Kang \& Choi (1999) foumd the critical value of rotation for vortex

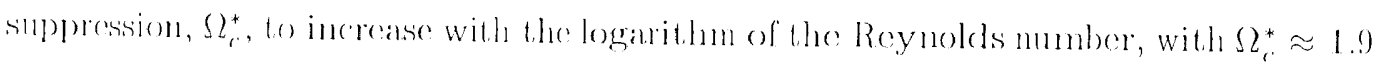
for Re - 160. Similar mumerical studies by Mithal \& Kumar (2003) at Re $=200$ and Stojkevic al al. $(2002)$ at $R e=100$ ane in agrecment, with estimates of $\Omega_{c}^{*} \approx 1.8$ and $\Omega_{*}^{*} \approx 1.91$, respertively. Vortex suppression has also becn observed at higher Reynolds mumbers, where the flow may he turbulent. Experiments by Diaz et al. (1983) at $R^{\prime}=9000$ found uo (e)herent structures associated with Kármán vortex shedeling for $\Omega_{r}^{*} \geq 2$. 
Why the rotation rate for vortex suppression on a cylinder is a factor of four greater than for a sphere is not clear and is somewhat counter-intulitive. Becantse the threedimensionality of the sphere makes it, less efficient, in generating circulation, one might expect that a comparatively fower rotation rate would be needed to suppress the wake of a cylinder.

Marked differences are also fonnd in the effece, that the rotation has on the average drage fores and the Strombal number of vortex shedding. For a spliere at moderate Reynoleds mumbers, an increase in the rotation rate leads to a greater average drag and the vortex shedeling Stroulat number also increases. Both these trends are reversed for the l.wo-dimensional flow past a rotating cylinder. Mittal \& Kunnar (2003), Kang

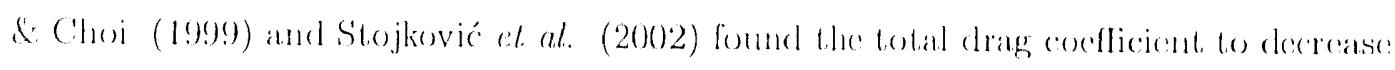
with increasing rotation mate, and for sulliciently high rotation rates the drag force

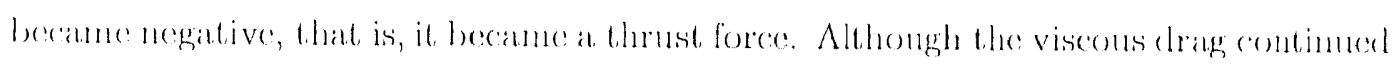
l. increase with rolation, the pressure drag which is dominame elecreased. At

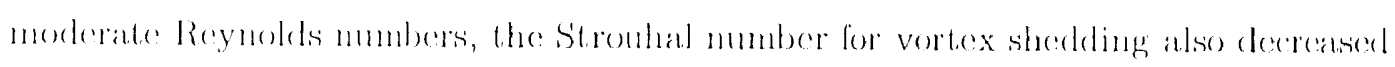
as the rotalion rate lends tewards $\Omega_{*}^{*}$. The differene between the two seenarios hats not bern noted in the literature, but it must somedow be asseciated with the theredimonsional geometry of the sphere. Mithal \& Kumar (20)(3) have pointed ont that. experimental and muncrical studles dealing with a rotaling cylinder show a converse:

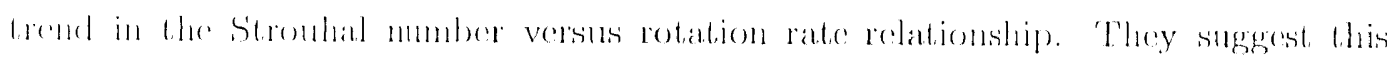
diflerence may be a result of the interaction between the vortex shedeling and the

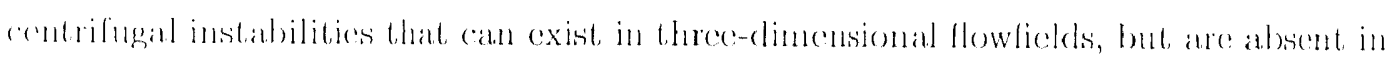

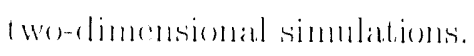

At, munch higher Reymolds mumbers $\left.(R e>10)^{4}\right)$, the lift, and drag forec ate ting on a rotating sphere are almost independent of Reynolds munber. One of the first instruInented experinents at, high Reynolds numbers was conducted by Maceoll (1928) using a b-inch diander sphere momted in the cross-stream of a wind-huned. The sphere wats fixed to a spindle that rotated the sphere via an electric motor. 'To minintize the 
cffects of the rotating spindle, it was shielded by a sleeve that spanned the width of the tunnel. Using a mechanical balance, the lift and drag forces were measured for rotation rates up to $\Omega^{*} \approx 6$ and Reynolds numbers in the range $R e \approx 3.2 \times 10^{4}$ to $10.8 \times 10^{4}$. $\dagger$ For low values of the rotation rate $\left(\Omega^{*}<0.5\right)$ the lift force was found to act in the opposite direction to that expected (the so-called negative Robins Magnus effect), while at higher values of rotation the lift force assumed the normal direction and roached a maximum at $\Omega^{*} \approx 2$ and remained relatively constant for higher valucs. The drag coefficient dropped in the range $\Omega^{*}=0$ to $\Omega^{*} \approx 1$, then increased to a maximum at $\Omega^{*} \approx 2$ and remained relatively constant thereafter.

The presence of a negative Robins - Magnus force at high Reynolds number and low rotation rates was confirmed by Davies (1949) and, later, Tanaka et al. (1990) and Aoki et al. (2003). Davies released spinning spheres into the cross-strcam of a wind-tunnel and used a trajectory technique to recover estimates of the drag and lift forces. For $R e \approx 9 \times 10^{4}$, a negative Robins Magnus effect was found for rotation ratcs loss than $\Omega^{*} \approx 0.5$. He attributed this behavior to the laminar-turbulent transition of the boundary layer. By rotating the sphere, the boundary layer on the retreating side experieneed a lower relative velocity and remained laminar. On the advancing side the higher relative velocity led to a higher local Reynolels number, which was sufficiont to trigger transition to turbulenee and cause the separation point to move downstream.

Both Tanaka el al. (1990) and Aoki el al. (2003) conducted wind-tumnel experiments similar to these of Maccoll, albeit, with more sophisticated measuring devices. Tanaka et al. (1990) moasured the lift and (lagg forces for Reynolds numbers in the range of $R e=6.08 \times 10^{4}$ to $1.40 \times 10^{5}$ and rotation rates less than $\Omega^{*} \approx 1.4$. $\Lambda$ t low $\Omega^{*}$, it, was found that the Robins Magnus force was positive and increased lincarly. $\Lambda$ s $\Omega 2^{*}$ increased, the lift coefficient decreased abruptly, before subsequently increasing. With

$\dagger$ Maccoll (1928) conducted experiments for cross-stream velocities in the range of 10 to 34 feet per second. The corresponding Reynolds numbers were not quoted, so they have been estimaled here by assuming the kinemalic viscosity for international standard conditions at sea level, namely $\nu=1.455 \times 10^{-5} \mathrm{~m}^{2} \mathrm{~s}^{-1}$. 
increasing Reynolds number, this abrupt drop in lift became more pronounced, such that for $R e>1.03 \times 10^{4}$ a negative Magnus force was observed.

For Ree $=16 \times 10^{4}$ and $\Omega^{*}=0.0$ to 0.38 , Aoki et al. (2003) found the lift and drag coefficients to follow comparable trends to those of Maccoll. Using a spark-tracing flow visualization method, the wake was seen to vector in the opposite direction to that expected from the classical Robins Magnus effect. When surface roughness was introduced, however, the wake deflection and lift force assumed the normal sign (a positive Robins Magnus effect) perhaps because the roughness causes the boundary layer over the entire surface to undergo a transition to turbulence. This supports the argument that the negative Robins Magnus effect is caused by a boundary layer transition on the advancing side only.

For the single case of $R e \approx 8.6 \times 10^{4}$ and $\Omega^{*}=4$, Maccoll took pressure surveys at several plancs downstream of a sphere using a multi-component pressure probe calibrated to measure both flow speed and direction. These moasurements revealed the formation of a pair of trailing vortices that were displaced towards the advancing side: of the sphere. It appears that the pressure difference leads to fluid following a curved path from high pressure to low pressure resulting in aswirling motion that gives rise to the trailing vortices.

\subsection{The flow past a cylinder in rotary oscillation}

The miform flow past, a rotationally oscillating sphere has, to the author's knowledge, never been studied. In contrast, the case of a rotationally oscillating cylinder has received considerable attention in recent times. The flow past a stationary circular cylinder shows a number of similarities to the flow past a stalionary sphere; however, there are also significant differences. A comprehensive review of the wake flow behind a stationary cylinder is provided by Williamson (1996). A brief review of the literature clealing with the rotationally oscillating cylinder is provided here. It is hoped that 
some understanding of the cylinder problem will provide insight into the mechanisms that shape the flowfield around a rotationally oscillating sphere.

Most studics into this problem have focused on controlling vortex shedding in a bluff body wake and have considered forcing frequencies in the vicinity of the natural Kármán vortex shedding frequency. It is well-known that rotational oscillation of a cylinder near the natural shedding frequency can cause resonance or so-called lockon. During lock-on, the vortex shedding frequency becomes synchronized with the frequency of forcing. This lock-on phenomena is also found for a cylinder undergoing linear transverse oscillation, a cylinder cxposed to excitation with an appropriate sound field, and a cylinder subject to periodic disturbance introduced in the incidence flow. A review of bluff body vortex shedding lock-on is given by Griffin \& Hall (1991).

To characterize the problem of forcing by rotary oscillation, two important forcing parameters are introduced: the maximum surface rotation speed normalized by the frecstream velocity, $\Omega_{o}^{*}$; and the cylinder oscillation frequency, $f_{f}$, or forcing Strouhal number, $S t_{f}=\int_{f} d / U_{\infty}$. Alternatively, the forcing amplitude may be specified in terms of a maximum angular displacement, $\Theta_{0}$. Most studies have considered sinusoidal forcing, in which case

$$
\Omega^{*}(t)=\Omega_{0}^{*} \sin \left(2 \pi \int_{s} l^{*}\right)
$$

It is more meaningful to express the forcing Stroulat number relative to the natural Kármán shedding Strouhal number, $S t_{n}$, in the form of the forcing frecuency ratio, $S t_{f} / S t_{n}$. It is also useful to define the vortex shedeling Strouhal mumber, $S t_{v}$, as this may be different to $S t_{n}$ when the cylinder undergoes rotary oscillation.

Choi at al. (2002) performed two-dimensional numerical simulations for $R e=100$, over the forcing parameter ranges $0.2 \leq \Omega_{\mathrm{o}}^{*} \leq 2.5$ and $0.02 \leq S t_{f} \leq 0.8\left(S t_{f} / S t_{n}=0.12\right.$ to 4.8). They found that the forcing frequency range over which lock-on occurred became wider as $\Omega_{0}^{*}$ was increased. Similar trends were observed in the numerical studies of Cheng el al. (2001a), Cheng et al. (2001b), Lu \& Sato (1996) and Mahfouz 
\& Badr (2000). At very low and very high $S t_{f} / S t_{n}$ and small forcing amplitudes, these studies found that the wake vortices were shed at a frequency close to the natural Kármán shedding frequency. However, as the forcing amplitude was increased beyond a threshold value, the vortices wore shed at the forcing frequency. This threshold value decreased as $S t_{f} / S t_{n}$ approached unity. A schematic representation of the lock-on boundary in the plane of $S t_{f} / S t_{n}$ versus $\Omega \Omega_{0}^{*}$ is shown in figure 1.12. Mahfouz \& Badr $(2000)$ also defined transition regions on the outskirts of the lock-on region, where the flow was characterized by an intermittent flow-switching between the lock-on and non-lock-on regimes.

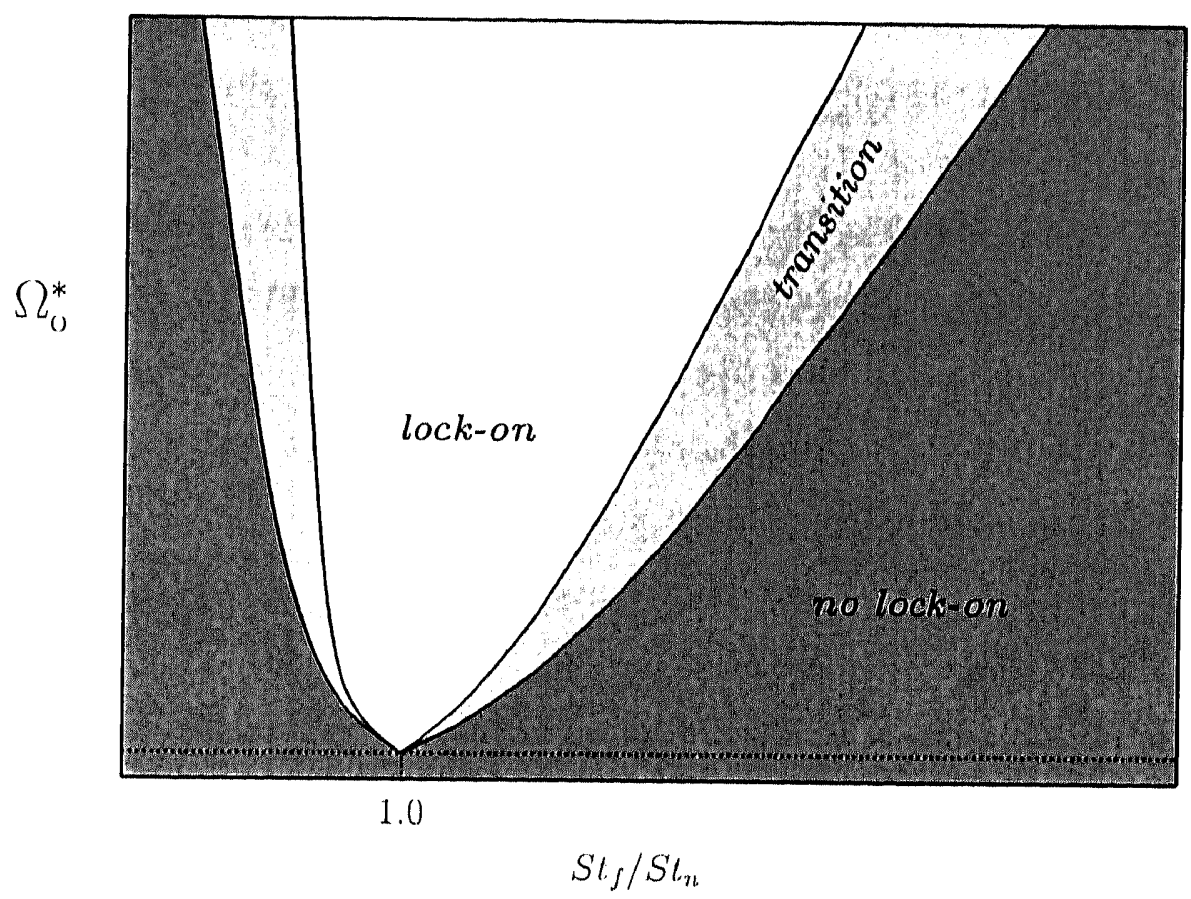

Figure 1.12: Schematic of the lock-on band for the forcing parameters $\Omega_{0}^{*}$ versus $S l_{s} / S l_{n}$.

In the non-lock-on regime, the vortices shed from the cylinder are of unequal strength, unlike those behind a stationary cylinder. This phenomenon is a result of the interaction between the unsteady flowficld and the oscillating cylinder. Cheng et al. 
(2001a) noted that for moderate forcing frequency ratios below and above lock-on, a weak vortex is shed from the cylinder every two to threc cycles. Because the flowficld is not synchronized with the cylinder motion, the relative velocity between the fluid and the cylinder surface secs a significant decrease every two to three shedding cycles. The decrease in relative velocity leads to a decrease in the vorticity generated at the boundary and thus the strength of the vortex that is formed and shed into the wake. The uncqual strength of the shed vortices is reflected in a modulated wave form for the lateral lift force. Choi et al. (2002) reported modulation to occur over a large range of $S t_{f} / S t_{n}$ outside the lock-on range and derived the following approximate relation for the modulated Strouhal number, $S t_{\text {mod }}$, in terms of a linear combination of $S l_{\text {, }}$ and $S t_{v}$ :

$$
S t_{m o l} \approx n \times S t_{f}+m \times S t_{v}
$$

where $n$ and $m$ are integers. As an example, they illustrate that for $R e=100$ and $S t_{f}=0.5$, the vortex shodding and modulation frequencies are $S t_{v}=0.1537$ and $S l_{\text {mod }}=0.0394$, respectively. Since $S t_{f} \approx 3 S t_{\eta}$, this corresponds to $n=1$ and $m=-3$, which, from equation $(1.7)$, gives $S t_{\text {moll }} \approx 0.0389$ which is in agreement, with the value of 0.0394 obtained from simulation.

As the forcing frecuency approaches the natural shedding frequency, the frecpuency of vortex shedeling shifts and becomes synchronized with the forcing frecuency. During lock-on the force histories clisplay a single frequency. The fluctuating amplitude: of the lift coofficient and average drag coefficiont increase towards a maximmm noar $S l_{s} / S l_{n} \approx 1 .(0$. Cheng et al. $(2001 \mathrm{a})$ argued that during lock-on, synchronization of the fluid and cylinder learls to the greatest relative velocity at the surface. The fluid gains additional vorticity in this regime and leads to the formation of stronger vortices, which is reflected by anarked increase in the fluctuating lift and drag coefficient. Back \& Sung (1998) applicd a two-dimensional DNS for $R e=110,0.11 \leq S t_{f} \leq 0.22$ and $\Theta_{0}=15,30$ and 60 degrecs. They also found that the peak valucs are shifted to slightly 


\subsection{THE FLOW PAST A CYLINDER IN ROTARY OSCILLATION}

lower values of the forcing frequency with increased forcing amplitude -.... in agreement with the numerical results of Choi et al. (2002) for $R e=100$ and Cheng et al. (2001a) for Re $=1000$. Choi et al. (2002) found that the peak drag and lift cocfficionts shift towards the lower frequency boundary of the lock-on regime as the forcing amplitude is increased. Both the drag and lift decrease with increasing forcing frequency, reaching a local minimum near the high frequency lock-on boundary before increasing again. This local minimum in the drag cocfficiont decreases with increasing forcing amplitude ancl, for a sufficiontly large forcing amplitude, may reach a value less than that for a stationary cylindor.

The phase relationship between the wake formation cycle and the cylinder rotation also seces a shift as the forcing frequency is increased towards the natural Kármán sheckling frecpuency. For the forcing amplitude $\Theta_{0}=30$ degrees, Back \& Sung (1998) found that, when $S t_{f}$ is sufficiontly lower than $S t_{n}$, cylinder rotation leads to a vortex being formed on the retreating side of the cylinder. This structure rolls up in the same direction as the cylincler rotation and leads to a suction pressure on the retreating side of the cylinder. In the neighborhood of $S t_{f} \approx S t_{n}$, a switch occurs in the vortex formation process and a wake vortex is formed on the advancing side of the cylinder with a circulation that, is in the opposite direction to the cylinder rotation. This phase shift has been reported in other numerical studies and also in the experimental study of Filler a al. (1991). Filler et al. considered $250 \leq R e \leq 1200,0.5 \leq S t_{f} / S t_{n} \leq 8$ and the relatively low forcing amplitudes of $0.005 \leq \Omega_{0}^{*} \leq 0.03$. A rapid change in the vortcx formation was observed near the natural Karmán shedding frecuency. As the Kármán frefuency was crossed, the vortex formation cycle essentially switched sides with respece, to the cylinder motion. As the forcing frequency was increased further, the phase relationship) varied in an essentially lincar fashion. Chou (1997), using DNS for $R e=1000$, found that increasing the forcing amplitude increased the phase angle; while, for a given forcing amplitude, the phase angle decreased approximatcly lincarly as $S t_{f} / S t_{n}$ was increased towards lock-on. As the frequency of forcing increased, the 
longitudinal spacing between the large-scale vortices shed into the wake varicd inversely with $S t_{f} / S t_{n}$, as also noted by Mahfouz \& Badr (2000). Chou (1997) quantificd these observations by measuring the longitudinal spacing between like-signed structures and found a lincar correlation with frequency. An increase in the forcing amplitude incrcased the spacing betwecn consecutive like-signed structures.

Intcrestingly, the switch in vortex formation phase for forcing near the Kármán shedding frequency is also observed for a cylinder in transverse oscillation. Experimentally, Ongoren \& Rockwell (1988) observed that the phase of the initial vortex formation, relative to the cylinder displacement, switched by approximatcly 180 degrecs ncar $S t_{f} / S t_{n} \approx 1$. In light of such findings, Tokumaru \& Dimotakis (1991) argued that the mechanisms that control the wake dynamics are largely generic, independent of the details of forcing. For the unforced case, vorticity is introduced into the wake from separation points that are essentially non-varying. Forcing the flow loads to unsteady scparation. Under these conditions, the natural shedding mochanism is replaced by a cyclic process of gathering vorticity close to the surface and releasing it. into the flow as a vortical structure.

The decrease in the mean drag coeflicient and lift fluctuations for $S l_{f} / S t_{n}$ above the lock-on range coincide with a narrowing of the wake. Taneda (1978a), by means of an (xperimental flow visualization for $R e=30$ t.0 300 and $S t_{f}=0$ 1.0 55, observed that increasing the foreing frecuency reduced the size of the wake region. Nbove at critical forcing frecuency, the wake was completely suppressed and the flow resembled the potential flow around a non-oscillating cylinder. This critical Strouhal number was found to be insensitive to Reynolds number over the range tested. However, as noted by Shicls \& Leonard (2001), these results have never been validated. Tokumaru \& Dimotakis (1991) experimontally investigated the wake of a rotationally oscillating circular cylinder for $R c=1.5 \times 10^{4}$ and $0.17 \leq S t_{\delta} \leq 3.3$. Using dye flow visualization and wake measurements using Laser Dopplor Anemometry, an ovcrall narrowing and widening of the wake was observed by varying the frequency and amplitude of cylinder 
oscillation. A minimum wake displacement area was found in the neighborhood $\Omega_{\mathrm{o}}^{*} \approx 3$ and $S t_{f} \approx 1$ (or $\left.S t_{f} / S t_{n} \approx 4.7\right) . \dagger$ For $R e=1.5 \times 10^{4}$, Tokumaru \& Dimotakis (1991) estimated the drag coefficient from wake deficit measurements. For $\Omega_{0}^{*}=2.0$ the estimated drag coofficiont showed a minimum at $S t_{f} \approx 1.0$, which was a factor of six lower than for the unforced case.

In an attempt to verify the drag reduction reported in the experimental study of Tokumaru \& Dimotakis (1991), Shicls \& Leonard (2001) applied a two-dimonsional, high-resolution viscous vortex method over the Reynolds number range $R e=150$ to 15000. For $R e=15000, \Omega_{0}^{*}=2.0$ and $S t_{f}=1.0$, Tokumaru \& Dimotakis found the drag coefficient to fall to $C_{D} \approx 0.2$, compared with an unforced value of $C_{D} \approx 1.4$. Using the same parameters, Sheils \& Leonard predicted $C_{D} \approx 0.43$. double the value reported by Tokumaru \& Dimotakis. In light of the assumption of two-dimensionality and what appears to be marginal resolution, the simulations serve to verify the large (Irop) in elrag coefficient observed by Tokumaru \& Dimotakis. The results also indicate that, the narrowing of the wake is associated with a boundary layer bursting from alternate sides of the cylinder for cach oscillation half-cycle. This boundary layer bursting leads to the formation of multipole mushroom-like vorticity structures, which are then convected downstream and lead to a delay in the time-averaged separation and an ansociated drag reduction.

Tokumaru \& Dimotakis (1991) classified the flow into four different regimes (modes I IV) in order of increasing forcing frequency. Examples of their water tunnel flow vistalization results are presented in figure 1.13 for cach regime. Here dye is used as the flow tracer and is injected at the surface of the cylinder. In mode I, $S t_{f} \approx S t_{n}$ and the cylinder releases two like-signed vortices into the wake per half-cycle, the second vortex being induced by the large shear caused by the first. Choi et al. (2002) reported

$\dagger$ Tokumaru \&. Dimotakis (1991) did not quote the natural Kármán shedding frequency, $S t_{n}$. Here the non-dimensional forcing frequency, $S t_{f} / S t_{n}$, is estimated using the experimentally derived curve-fit of Roshko (1954). Using the hot-wire measurement technique, Roshko measured $S t_{n}$ from $R e=40$ to 10000 . The best-fit line to this data, $S t_{n}=0.212(1.0-12.7 / R e)$, gives an extrapolated value of $S t_{n}=0.212$ for $R e=1.5 \times 10^{4}$. 
the wake structure in this regime to be Reynolds number dependent. Their numerical studies at $R e=100$ did not show a second like-signed vortcx, while an cxploratory simulation at $R e=1000$ showed excellent agreement with the finding of Tokumaru \& Dimotakis.

In mode II, the wake becomes synchronized with the cylinder forcing and persists far downstream of the cylinder. In mode III, the near-wake structure is synchronized with the cylinder forcing, but becomes unstable further downstream and evolves into a structure with lower frequency. In this regime, Choi et al. (2002) showed that small scalcs appear in the shear layer as a result of the high forcing frequency. These small scales then undergo a series of vortex merging processes, until the wake recovers to a large-scale antisymmetric structure resembling the Kármán vortex strect, with a characteristic frequency close to $S t_{n}$. The forcing frequency was found to govern the number of vortices involved in the vortex merging sequenee, while the forcing amplitude determined the order of vortex merging during one period. This sequence of vortex merging is also described by Ongoren \& Rockwell (1988) for a cylinder in transverse oscillation.

Finally, in mode IV, the small scales generated at the surface remain confined to the: shenr layers. The structures coalesee as they are convected downstream and form larger scales before being shed into the wake. These large scales resemble the Kármán vortex street shed from a stationary cylinder. Back \& Sung (1998) noted that in this regime, the small shear layer vortices retard the onset of large-seale vortex formation in the downstream wake. In effect, this leads to a delay in gross separation and a narrowing of the wake, which explains the drag drop at forcing frequencies above the lock-on range. Tokumaru \& Dimotakis (1991) also conducted exploratory experiments using a cylinder whose surface was roughened and a smooth cylinder at a lower Reynolds number of $3.3 \times 10^{3}$. In all cases the flow visualization results showed very similar trends, which led the authors to conclude that the various wake regimes are relatively independent of the Reynolds number. Lu \& Sato (1996) perform two-dimensional 


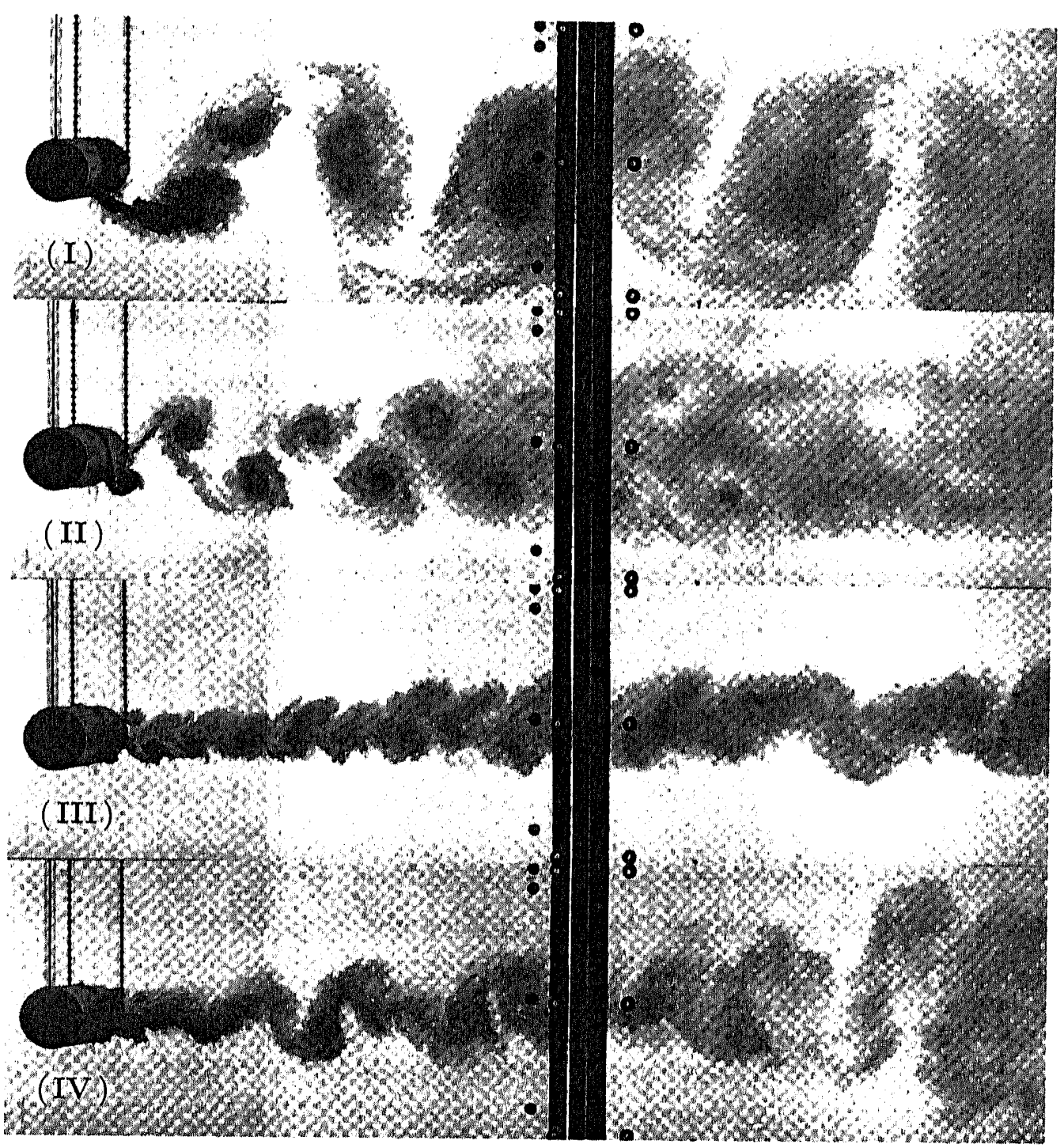

Figure 1.13: Flow visualization data of Tokumaru \& Dimotakis (1991) for tho wake of a circular cylinder in rotary oscillation at $R e=1.5 \times 10^{4}$. The cases shown illustrate wake modes I to IV defined by the authors: (I) $S t_{f}=0.2, \Omega_{0}^{*}=2$; (II) $S t_{\rho}=0.5$, $S \Sigma_{0}^{*}=8 ;$ (III) $S t_{f}=1.1, \Omega_{\circ}^{*}=8 ;($ IV $) S t_{f}=2.0, \Omega_{0}^{*}=8$. 
numerical simulations at $R e=200,1000$ and 3000; they also found the effect of forcing on the large-scalc vortex structures to be insensitive to the Reynolds number.

For $R e=100$, Choi et al. (2002) showed that the various wake regimes could also be classified in accordance with the modes defined by Tokumaru \& Dimotakis (1991) for a much higher Reynolds number. They noted that in this classification, mode I and II belong to a lock-on regime, while in mode IV, the wake is not locked onto the forcing frequency. Mode III can be classified into either a lock-on or nonlock-on regime, depending on the wake probing position. Sensing in the near-wake would suggest the wake to be in a lock-on regime, while sensing further down stream would indicate a non-lock-on regime. In a number of studies, the time-history of the lift cocfficient is used to classify the flow, in which case mode III is classified as lock-on. The importance of the sensing location is highlighted by the expcrimental findings of Filler et al. (1991), where a hot-film is placed in the shear layer downstream of the cylinder. Bolow Re $=500$, only the Kármán vortex shedding frequency was detected, while at higher Reynolds numbers the forcing could also affect the shear layer vortices. For example, at, $R e=925$, the shear layers were initially laminar and wrapped into the Kármán vortices. However, forcing at $S t_{f} \approx 3.0 S t_{n}$ and $\Omega_{0}^{*}=0.025$ led to the formation of shear layer vorlices that are similar to those observed for a stalionary cylinder at higher Reynolds numbers. 


\subsection{Aims and outline}

A number of studies have considered the problem of a sphere in steady transverse rotation, immersed in a uniform moderate Reynolds number flow. Despite these efforts, lit,te is known about the three-dimensional nature of the flowfield and the mechanisms that drive the varions wake transitions. It is the author's understanding that the problem of a sphere undergoing oscillatory rotation, about an axis transverse to the frecstream flow, has never been considered. In an effort to accquire this understanding, the following aims were formulated:

- Modify and validate existing numerical algorithms to simulate the problem of a smooth sphere rotating in a uniform cross-flow.

- Critically cvaluate the commonly used vortex extraction methods to facilitate the identification and tracking of vortical structures.

- For a sphere in stcady rotation, compute the field at moderate Reynolds numbers and rotation rates, namely, Reynolds numbers in the range $R e=100$ to 300 and non-climensional rotation rates in the range $\Omega^{*}=0.00$ to 1.00 .

- Ior a sphere undergoing rotary oscillation, compute the flowfield for $R e=300$, the rotation rate amplitude $\Omega_{0}^{*}=0.50$ and forcing frequency ratios in the range $S l_{f} / S l_{n}=0.00$ lo 3.00

- Analyze the data set, with a focus towards understanding the nature of the various wake transitions.

A broal outline of the ensuing chapters is as follows:

- Chapter 2 describes the numerical algorithms used in the Navier Stokes solver. The discussion focuses on aspects of the solver which are peculiar to the problem.

- In chapter 3 the solver is validated against available numerical and experimental data for both a stationary and rotating sphere in a uniform cross-flow. 
- The study makes extensive use of frame-invariant vortex identification methods.

There has been much debate as to the most suitable candidate for consistent, and unambiguous identification of coherent structures. In chapter 4 the various methods and their merits are described.

- Chapter 5 presents numerical results for $R e=100$ to 300 and stcady rotation at $\Omega^{*}=0.00$ to 1.00. Emphasis is placed on the topological transitions of the wake structure with increasing Reynolds number and sphere rotation rate. The mechanisms which drive the transitions are described.

- In chapter 6 the flowficld around a sphere undergoing sinusoidal oscillation about an axis transverse to the frestream flow is investigated for $R e=300$. The forcing amplitude is restricted to $\Omega_{0}^{*}=0.50$ and the forcing frequencies cover the range $0.0 \leq S t_{\rho} / S t_{n} \leq 3.0$.

- Conclusions and suggestions for further work are presented in chapter 7. 


\section{Chapter 2}

\section{Numerical formulation}

The incompressible Navier Stokes equations are solved dircetly for the primitive variables in a spherical polar coordinate system. The equations are spatially discretized using a Fourier Chebyshev collocated method that employs a restricted double Fourier series in the wall-tangential directions and a Chebyshev discretization in the wallnormal direction. Time advancoment is via a two-step fractional-step method that is second-order accurate for the velocity field and may be formulated to be first or secondorder accurate for the pressure ficld. The numerical scheme is based on that cmployed by Bagchi \& Balachandar (2002b) and Mittal (1999b) for the direct simulation of the flow past spheres and prolate spheroids, respectively. It has also boen used in a modilied form by Mittal (1995) and Mittal \& Balachandar (1996) to study the flow past clliptic and circular cylinders. This section describes the details of the numerical formulation. In particular, issues related to the accuracy of the fractional-step method, the treatment of the 'pole problem' and 'pole-parity problem' that arise on spherical griels and the efficient, solution of Helmholtz and Poisson equations are discussed.

\subsection{Governing equations and coordinate system}

We consider the motion of an incompressible, Newtonian fluid past a spherical particle. The particle is constrained to rotate about an axis normal to the frec-stream flow vector, both with a constant angular velocity and with a sinusoidally oscillating angular 
velocity. The fluid motion is fully described by the unsteady incompressible Navier Stokes equations, which take the form

$$
\frac{\partial u}{\partial t}+u \cdot \nabla u=-\nabla P+\frac{1}{R e} \nabla^{2} u
$$

and

$$
\nabla \cdot u=0
$$

Here the Reynolds number, $R e$, is defined based on the freestream velocity, $U_{\infty}$, the diameter of the sphere, $d$, and the kincmatic viscosity, $\nu$. All length scales are therefore non-dimensionalized by the sphere diameter, $d$, all velocity scales by the frec-stream velocity and all time scales by $d / U_{\infty}$. The static pressure, $P$, is non-dimensionalized by the freestream dynamic pressure, $\rho U_{\infty}^{2}$, where $\rho$ is the fluid density. The other parameters important in this problem are the non-dimensional angular velocity of the rotating sphere, $\Omega^{*}=\Omega d / 2 U_{\infty}$ and the non-dimensional forcing frequency, or forcing Strouhal number, $S t_{\rho}=\int_{\rho} d / U_{\infty}$. Here $\Omega$ is the angular velocity vector of the rotating sphere. and $\int_{f}$ is the forcing frequency when rotationally oscillating motion is considered.

To compute the flow around a spherical particle, it is natural to solve the governing cquations in a spherical polar coordinate system as presented in figure 2.1. A point in space is described by the coordinates $(r, \theta, \phi)$, which are referred to as the radial, tangential and azimulhal directions, respectively. The unit vectors in these respective directions are $\hat{e}_{r}, \hat{e}_{\theta}$ and $\widehat{e}_{\phi}$. A cartesian coordinate system, $(x, y, z)$, is also introduced to resolve forces and moments along and normal to the frestream flow direction. The frecstrean flow vector is aligned with the positive $z$-axis and the sphere angular velocity vector is directed along the positive $x$-axis. The transformation from a velocity vector in spherical coordinates, $\left(u_{r}, u_{0}, u_{\phi}\right)$, to cartesian coordinates, $\left(u_{x}, u_{y}, u_{z}\right)$, is given by

$$
\left\{\begin{array}{l}
u_{x} \\
u_{y} \\
u_{z}
\end{array}\right\}=\left[\begin{array}{ccc}
\sin \theta \cos \phi & \cos \theta \cos \phi & -\sin \phi \\
\sin \theta \sin \phi & \cos \theta \sin \phi & \cos \phi \\
\cos \theta & -\sin \theta & 0
\end{array}\right]\left\{\begin{array}{l}
u_{r} \\
u_{\theta} \\
u_{\phi}
\end{array}\right\} .
$$


In a spherical polar coordinate system, the non-linear convective terms in the $r, \theta$ and $\phi$ directions are

$$
\begin{aligned}
& (\boldsymbol{u} \cdot \nabla \boldsymbol{u})_{r}=u_{r} \frac{\partial u_{r}}{\partial r}+\frac{u_{\theta}}{r} \frac{\partial u_{r}}{\partial \theta}+\frac{u_{\phi}}{r \sin \theta} \frac{\partial u_{r}}{\partial \phi}-\frac{u_{\theta}^{2}+u_{\phi}^{2}}{r} \\
& (\boldsymbol{u} \cdot \nabla \boldsymbol{u})_{\theta}=u_{r} \frac{\partial u_{\theta}}{\partial r}+\frac{u_{\theta}}{r} \frac{\partial u_{\theta}}{\partial \theta}+\frac{u_{\phi}}{r \sin \theta} \frac{\partial u_{\theta}}{\partial \phi}+\frac{u_{0} u_{r}}{r}-\frac{u_{\phi}^{2} \cot \theta}{r}
\end{aligned}
$$

and

$$
(u \cdot \nabla u)_{\phi}=u_{r} \frac{\partial u_{\phi}}{\partial r}+\frac{u_{\theta}}{r} \frac{\partial u_{\phi}}{\partial \theta}+\frac{u_{\phi}}{r \sin \theta} \frac{\partial u_{\phi}}{\partial \phi} \frac{u_{r} \cdot u_{\phi}}{r}+\frac{u_{0} u_{\phi} \cot \theta}{r} .
$$

The diffusion terms are given by

$$
\begin{aligned}
& \left(\nabla^{2} u\right)_{r}=\nabla^{2} u_{r}-\frac{2 u_{r}}{r^{2}}-\frac{2}{r^{2}} \frac{\partial u_{\theta}}{\partial \theta}-\frac{2 u_{\theta} \cot \theta}{r^{2}}-\frac{2}{r^{2} \sin \theta} \frac{\partial u_{\phi}}{\partial \phi}, \\
& \left(\nabla^{2} u\right)_{\theta}=\nabla^{2} u_{\theta}+\frac{2}{r^{2}} \frac{\partial u_{r}}{\partial \theta}-\frac{u_{\theta}}{r^{2} \sin ^{2} \theta}-\frac{2 \cos \theta}{r^{2} \sin ^{2} \theta} \frac{\partial u_{\phi}}{\partial \phi}
\end{aligned}
$$

and

$$
\left(\nabla^{2} u\right)_{\phi}=\nabla^{2} u_{\phi}-\frac{u_{\phi}}{r^{2} \sin ^{2} \theta}+\frac{2}{r^{2} \sin \theta} \frac{\partial u_{r}}{\partial \phi}+\frac{2 \cos \theta}{r^{2} \sin ^{2} \theta} \frac{\partial u_{\phi}}{\partial \phi},
$$

where the scalar Laplacian takes the form

$$
\nabla^{2}=\frac{2}{r} \frac{\partial}{\partial r}+\frac{\partial^{2}}{\partial r^{2}}+\frac{\cot \theta}{r^{2}} \frac{\partial}{\partial \theta}+\frac{1}{r^{2}} \frac{\partial^{2}}{\partial \theta^{2}}+\frac{1}{r^{2} \sin ^{2} \theta} \frac{\partial^{2}}{\partial \phi^{2}}
$$

The continuty ecpuation may be written as

$$
\nabla \cdot \boldsymbol{u}=\frac{1}{r^{2}} \frac{\partial\left(r^{2} u_{r}\right)}{\partial r}+\frac{1}{r \sin \theta} \frac{\partial\left(\sin \theta u_{\theta}\right)}{\partial \theta}+\frac{1}{r \sin \theta} \frac{\partial u_{\phi}}{\partial \phi}=0 .
$$

Dinensionless force and torque coefficients are obtained by numerically integrating the viscous and pressure forecs over the sphere surface using the third-order Simpson's rule. For example, the foree coefficient resolved along the $z$-direction, also known as the drag coeflicient, is given by

$$
C_{D}=\frac{1}{\frac{1}{2} \rho U_{\infty}^{2} \pi\left(\frac{d}{2}\right)^{2}} \oint_{\partial \Gamma}(\widehat{n} \cdot \sigma) \cdot \widehat{e}_{\boldsymbol{x}} \mathrm{d} S
$$




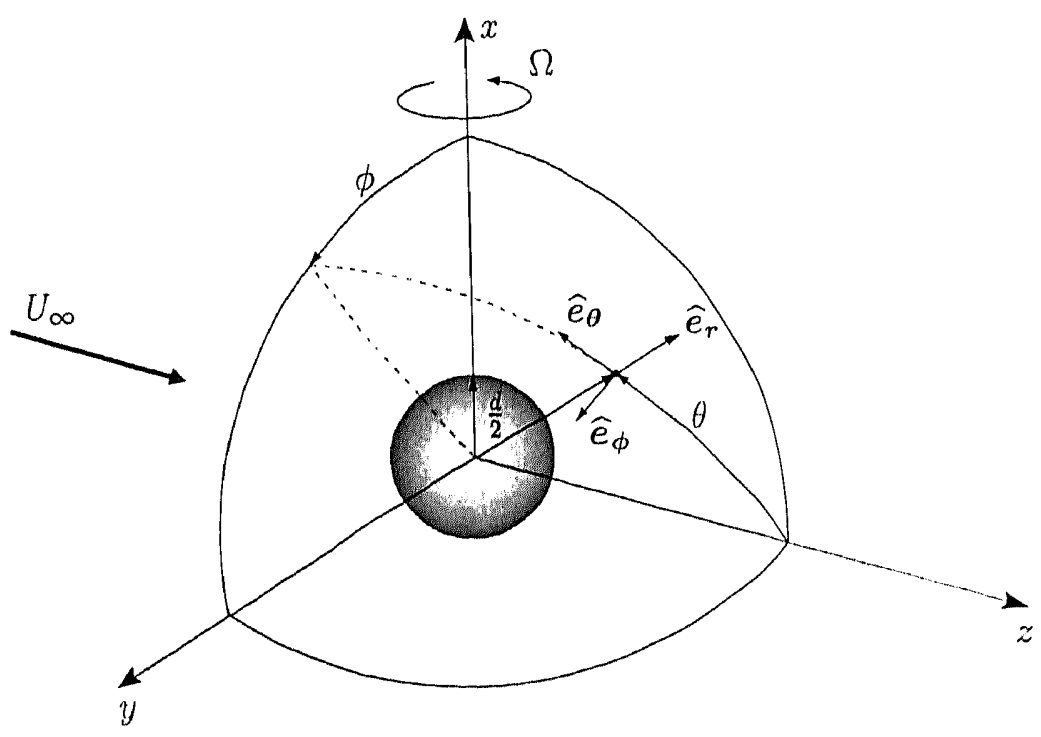

Figure 2.1: Problem goometry and coordinale system. The frocstream flow is along the $z$-axis and the sphere is constrained to rotate about the $x$-axis.

while the torque coefficient, about the $z$-axis is given by

$$
C_{M z}=\frac{1}{\frac{1}{2} \rho U_{\infty}^{2} \pi\left(\frac{d}{2}\right)^{2}} \oint_{\partial \Gamma}\left((\hat{n} \cdot \sigma) \times\left(\frac{d \hat{e}_{r}}{2}\right)\right) \cdot \hat{e}_{z} \text { d. }
$$

Expressions for the force coefficients in the x-direction, $C_{t, r}$, and $y$-direction, $C_{t y,}$, and momont coefficients in the x-direction, $C_{M x}$, and $y$-direction, $C_{M ! y}$, are ohtatined by replacing $\hat{e}_{z}$ in the above expressions with $\hat{e}_{x}$ and $\hat{e}_{y}$, respectively. Here $\hat{n}$ is a mil vector that is normal to the sphere surface, or indicates the sphere surface and dis is an clemental surface areat. $\sigma$ is the stress tensor, which may be writtent ats

$$
\sigma=-p I+\mu\left(\nabla u+\nabla u^{l}\right)
$$

where $I$ is the identity matrix, $\mu$ is the clynamice viscosity of the fluid and $\nabla u$ is the velocity gradient tensor, which in spherical coordinates is given as 


$$
\nabla u=\left[\begin{array}{ccc}
\frac{\partial u_{r}}{\partial r} & \frac{1}{r} \frac{\partial u_{r}}{\partial \theta}-\frac{u_{\theta}}{r} & \frac{1}{r \sin \theta} \frac{\partial u_{r}}{\partial \phi}-\frac{u_{\phi}}{r} \\
\frac{\partial u_{\theta}}{\partial r} & \frac{1}{r} \frac{\partial u_{\theta}}{\partial \theta}+\frac{u_{r}}{r} & \frac{1}{r \sin \theta} \frac{\partial u_{\theta}}{\partial \phi}-\frac{u_{\phi} \cot \theta}{r} \\
\frac{\partial u_{\phi}}{\partial r} & \frac{1}{r} \frac{\partial u_{\phi}}{\partial \theta} & \frac{1}{r \sin \theta} \frac{\partial u_{\phi}}{\partial \phi}+\frac{u_{r}}{r}+\frac{u_{\theta} \cot \theta}{r}
\end{array}\right]
$$

Equations (2.1) to (2.11) and equation (2.15) were derived using the expressions for common vector differential quantities, which are presented in Appendix 2 of Batchelor $(200))$.

\subsection{Spatial discretization}

The coordinate system comprises a radial, tangential and azimuthal direction $(r, \theta, \phi)$, where:

$$
d / 2 \leq r \leq D_{0} / 2, \quad 0 \leq \theta \leq \pi \text { and } 0 \leq \phi \leq 2 \pi \text {. }
$$

The discretization in cach of these directions is described in turn. In the raclial clirection, the infinite flow domain is truncated to a finite extent, where $D_{0}$ is the diameter of the outer boundary of the computational domain. A Chobyshev expansion is used in this diretion and the Gauss Lobatho collocation points, on the natural domain $[-1,1]$, are given by

$$
x_{i}=-\cos \left[\frac{\pi(i-1)}{N_{r}-1}\right] \text { for } i=1,2, \ldots, N_{r},
$$

where $N_{r}$ is the number of collocation points in the radial direction. To efliciently reselve the high gradients in the sphere shear layer and near-wake, this distribution of points is stretched to chuster collocation peints near the sphere surface. The amoment of stretching is tailored to consure at least twelve to fiftecn collocation points are placed within the boundary layer, while also ensuring that there is an adequate decay of the Chebyshev expansion coeflicients. The stretching function is given as

$$
\widetilde{x}_{i}=c_{0}+c_{1} x_{i}-c_{0} x_{i}^{2}+c_{2} x_{i}^{3}
$$


where

$$
c_{1}=\frac{1}{2}\left(-x_{\min }+2 c_{0}+3\right) \text { and } c_{2}=\frac{1}{2}\left(x_{\min }-2 c_{0}-1\right)
$$

The parameter $c_{0}$ controls the extent to which the grid is clustered towards the sphere surface, with $c_{0}=0.0$ resulting in no clustering and increasing negative values producing a progressively fincr grid near the sphere surface. The parameter $x_{\text {min }}$ controls the grid spacing at the wall, with increasing values producing a coarser grid. For all simulations presented in this study, $c_{0}=-0.6$ and $x_{m i n}$ is in the range (0.2 to 0.3 . Finally, this distribution of collocation points is mapped to the physical domain via

$$
r_{i}=\frac{1}{2}\left(\frac{D_{0}}{2}+\frac{d}{2}\right)+\frac{1}{2}\left(\frac{D_{0}}{2}-\frac{d}{2}\right) \widetilde{x}_{i} .
$$

The radial stretching function is illustrated in figure $2.2(a)$ and $2.2(b)$ for various values of $c_{0}$ and $x_{\text {tnin }}$.

In the directions parallel to the wall, surface harmonics are a natural choice of basis function. Their main advantages are the absence of a coordinate singularity at. the poles and their uniform resolution propertics over the whole surface of the splecre. However, their disadvantage lies in the absence of a last transform, which can make them more computationally expensive to use. Instead, a restricted double Fourier series is cmployed in the wall-parallel directions, for which a fast transform can be cmployed. Spectral methods based on surface harmonies refuire $O(N)$ operations per

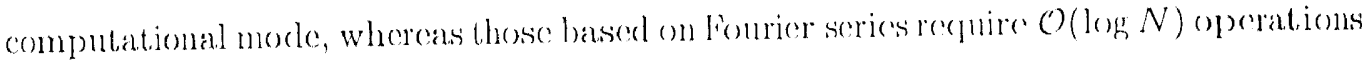
when a fast Fourior transform is used. This leats to a signilicant compulational saving as the number of collocation points, $N$, becomes large. The main disadvantage in using a double Fourior series lies in the presence of the 'pole problem', which results in a severe time stepping restriction. This is due to the high clustering of collocation points near the poles. A critical evaluation of spherical harmonic functions and Fourier scries for spatial discretization in spherical geometries is provided by (Orzag (1974).

In the azimuthal (or $\phi$-direction), the primitive variables are intrinsically periodic over $2 \pi$ and a Fouricr expansion is the natural choice for this direction. The collocation 
points are equally spaced and given as

$$
\phi_{k}=\frac{2 \pi(l-1)}{N_{\phi}} \text { for } l=1,2, \ldots, N_{\phi}
$$

where $N_{\phi}$ is the number of collocation points in this direction.

In the tangential direction, the primitive variables are also intrinsically periodic over $0 \leq \theta \leq 2 \pi$, however, the computational domain is fully defined over $0 \leq 0 \leq \pi$. As a result, the tangential expansion must be constructed to obey cortain behavioral boundary conditions in order for it to be compatible with the azimuthal expansion. The expansion must also cope with the so-called 'pole parity' problem that arises in spherical coordinates. This refors to the tangential and azimuthal components of a vector changing sign as the poles $(\theta=0$ and $\theta=\pi)$ are crossed. The tangential and azimuthal derivatives of a scalar also change sign, while the radial component of a vector and the raclial derivative of a vector are continuous across the poles. parity problem and the properties of an aceptable expansion are discussed by Orzag (1974) and Boyd (1989). The expansions must obey the pole parity conditions and be inlinitely differentiable to avoid slow convergence of the numerical scheme and any emergence of Cibbs phenomena. Below are the final form of the expansions used in the solver

$$
\begin{aligned}
& s=\left\{\begin{array}{l}
\sum_{p=0}^{N_{r}-1} \sum_{m=0}^{N_{\theta} \cdots 1} \sum_{k=-N_{\phi} / 2}^{N_{\phi} / 2}\left(k_{p m k} T_{p}(r) \cos (m, \theta) e^{\mathrm{i} k \phi} \quad \mathrm{even} k\right. \\
\sum_{p=1}^{N_{r}-1} \sum_{m=1}^{N_{p}} \sum_{k=N_{\phi} / 2}^{N_{\phi} / 2} \alpha_{p m k} T_{p}(r) \sin (m, \theta) e^{\mathrm{i} k \phi \phi} \text { odd } k,
\end{array}\right. \\
& u_{r}=\left\{\begin{array}{l}
\sum_{p=0}^{N_{r}-1} \sum_{m=0}^{N_{g}-1} \sum_{k=-N_{\phi} / 2}^{N_{\phi} / 2} \beta_{p m k} T_{p}(r) \cos (m \theta) e^{\mathrm{i} k \phi \phi} \text { cven } k \\
\sum_{p=0}^{N_{r}-1} \sum_{m=1}^{N_{\theta}} \sum_{k=-N_{\phi} / 2}^{N_{\phi} / 2} \beta_{p m k} T_{p}(r) \sin (m \theta) e^{\mathrm{i} k \phi} \text { odd } k,
\end{array}\right.
\end{aligned}
$$

$\dagger$ A scalau describes a field which has a single value at each point in space and is invariant under a coordinate transformation. 


$$
\begin{aligned}
& u_{\theta}=\left\{\begin{array}{lll}
\sum_{p=0}^{N r-1} \sum_{m=1}^{N_{\theta}} \sum_{k=-N_{\phi} / 2}^{N_{\phi} / 2} \gamma_{p m k} T_{p}(r) \sin (m \theta) c^{\mathrm{i} k \phi} & \text { even } & k \\
\sum_{p=0}^{N r-1} \sum_{m=0}^{N_{\theta}-1} \sum_{k=-N_{\phi} / 2}^{N_{\phi} / 2} \gamma_{p m k} T_{p}(r) \cos (m \theta) e^{\mathrm{i} k \phi} & \text { oddl } \quad k
\end{array}\right. \\
& u_{\phi}=\left\{\begin{array}{l}
\sum_{p=0}^{N_{r}-1} \sum_{m=1}^{N_{\theta}} \sum_{k=-N_{\phi} / 2}^{N_{\phi} / 2} \delta_{p m k} T_{p}(r) \sin (m \theta) e^{\mathrm{i} k \phi} \quad \text { even } k \\
\sum_{p=0}^{N_{r}-1} \sum_{m=0}^{N_{\theta}-1} \sum_{k=-N_{\phi} / 2}^{N_{\phi} / 2} \delta_{p m k} T_{p}(r) \cos (m \theta) c^{\mathrm{i} k \phi} \quad \text { oclel } k,
\end{array}\right.
\end{aligned}
$$

where $s$ represents a scalar quantity such as the pressure fielel. $u_{r}, u_{0}$ and $u_{\phi}$ are the velocity components in the $r, \theta$ and $\phi$ directions, respectively. $T_{p}$ represents the $p^{\text {th }}$ Chebyshev polynomial. $m$ and $k$ are the wavenumbers in the $\theta$ and $\phi$ directions, respectively, $\alpha, \beta, \gamma$ and $\delta$ are the expansion coefficients and are a function of $p$, mand $k$. These expansion functions are shown to be acceptable, by cxample, in $\Lambda_{\text {ppendix }} A$.

The collocation points in the tangential direction are defined as

$$
\tilde{\theta}_{j}=\frac{\pi(j-1 / 2)}{N_{\theta}} \text { for } j=1,2, \ldots, N_{\theta}
$$

where $N_{0}$ is the number of grid points in the tangential direction. The collocation points are shifted to avoirl the ' $1 /$ sing' singularity that orecurs when the geverning ecplations are evaluated directly on the polar axis $(\theta=0$ and $\pi)$. In order to ceffeciently resolve the structures in the sphere wake, this miform clistribution of collocation points is clustered towards the downstream pole $(\theta=0)$ using the mapping

$$
\theta_{j}=\tan ^{\cdots 1}\left(\frac{\sin \tilde{\theta}_{j}\left(1-\Theta^{2}\right)}{\cos \tilde{\theta}_{j}\left(1+\Theta^{2}\right)-2 \Theta}\right) .
$$

The langential stretching function is illustrated in figure $2.2(c)$ for various values of $\theta$. The parameter $\Theta$ controls the extent of the grid clustering, with increisingly negative values clustering points in the wake and increasingly positive values chuster points towards the upstream polc. For all simulations presented in this study, the value $\Theta=-0.35$ was used. 

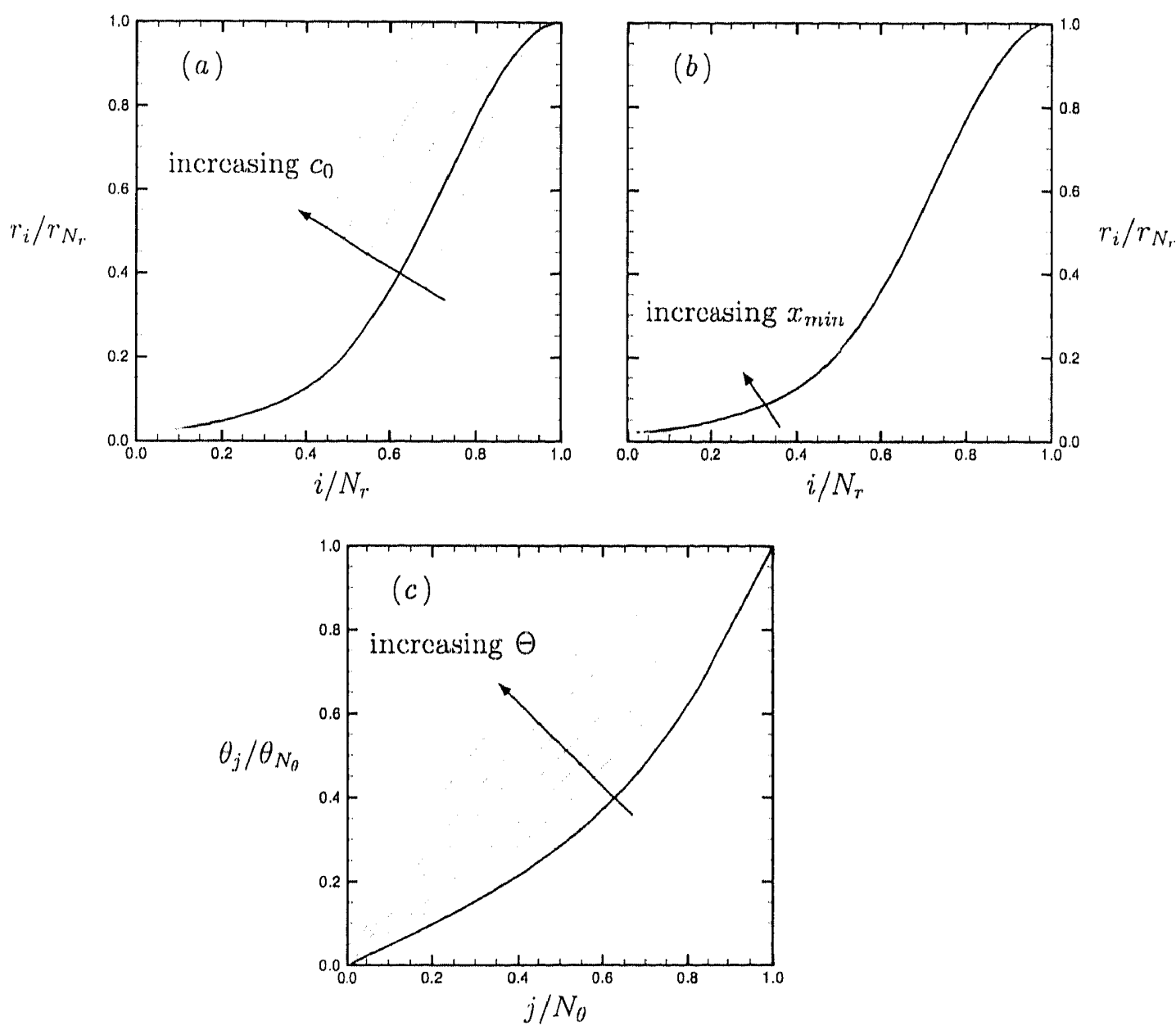

Fignre 2.2: Stretching functions used in the radial and tangential directions: (a) The effect of en on the radial grid stretching. $c_{0}=-0.9,-0.6,-0.3,0.0$ and 0.3 are shown, with $x_{\min }=0.3$. (b) The effect of $x_{\min }$ on the radial grid stretehing. $x_{\min }=-(0.3$, $-\left(0.0,0.3\right.$ and 0.6 are shown, with $c_{0}=-0.6$. (c) The effect of $\Theta$ on the tangential grid stretching. $\Theta=0, \pm 0.1, \pm 0.2$ and \pm 0.35 are shown. The solid black lines correspond to stretching parancters used in this study, namcly $c_{0}=-0.6, x_{\text {min }}=0.3$ and $\Theta=-0.35$.

A typical computational grid is shown in figure 2.3. A sector of the domain has ben removed to show the griel point distribution through the clomain. The magnified view shows the chustering of collocation points near the sphere surface and wake region. The collocation points are defined at the vertices of the hexahedral cells. The high grid clustering near the sphere poles $(\theta=0$ and $\theta=\pi)$ requires a prohibitively small limestep in order to remain within the convective and diffusive stability limits of the solver. 
This time-step restriction is alleviated somewhat by applying a filter that truncates the high azimuthal modes near the poles.

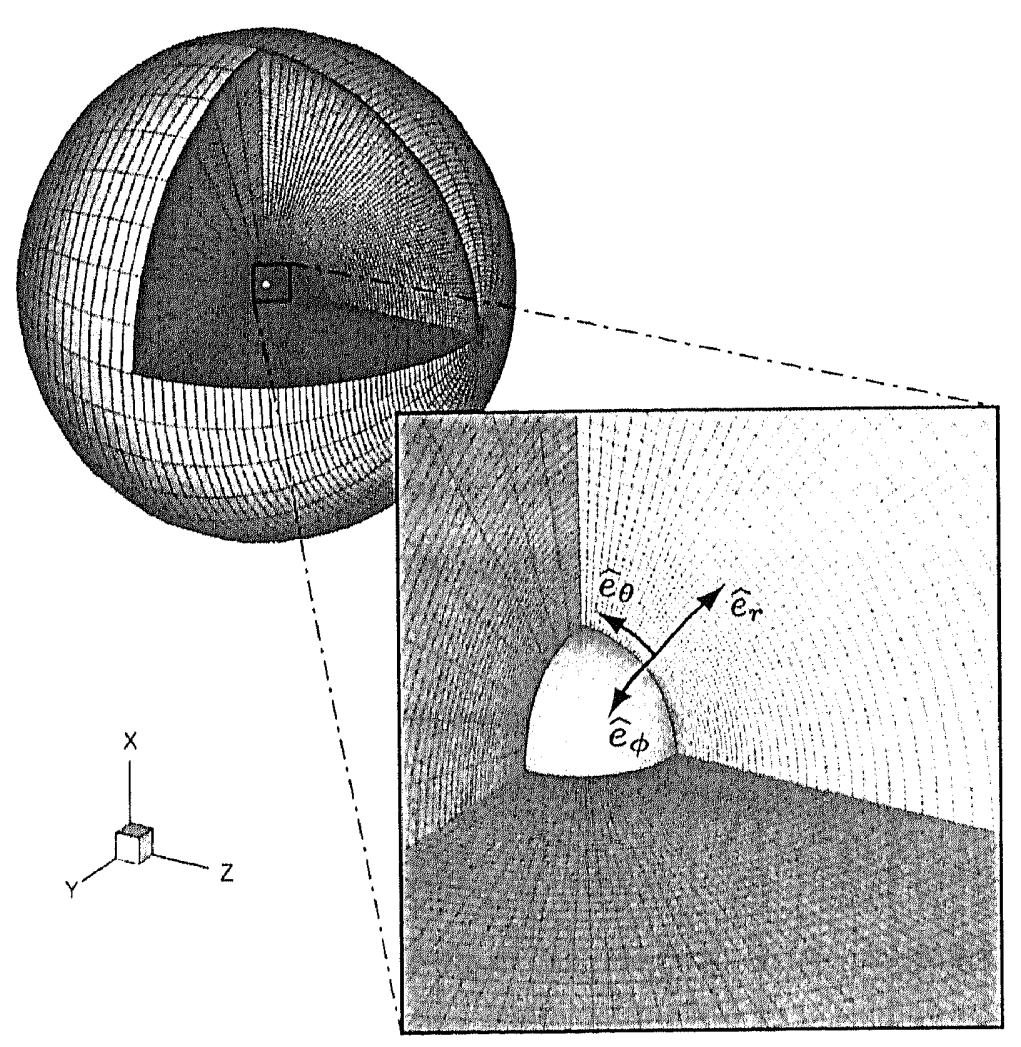

Figure 2.3: ^ representative compulational grid. The collocation peints are defined at the vertices of the cells.

\subsubsection{The pole problem}

Fignre 2.4 shows a schematic representation of the collocation points on the sphere surface and along a werlge emanating from the downstrean pole. Althomgh the azimuthat grid spacing $(\Delta \phi)$ is constant, the spatial resolution in the azimuthal clirection is non-1niform and is given by $r \sin \theta \Delta \phi$. Ss a result, the spatial azimuthal resolulion increases both as the poles are approached and as the surface of the splecre is approached. Numerically, this increase in spatial resolution can impose severe viscous and convective time-stepping stability limits. The viscous stability constraint due to the non-uniform resolution is alleviated by the implicit treatment of the radial and az- 


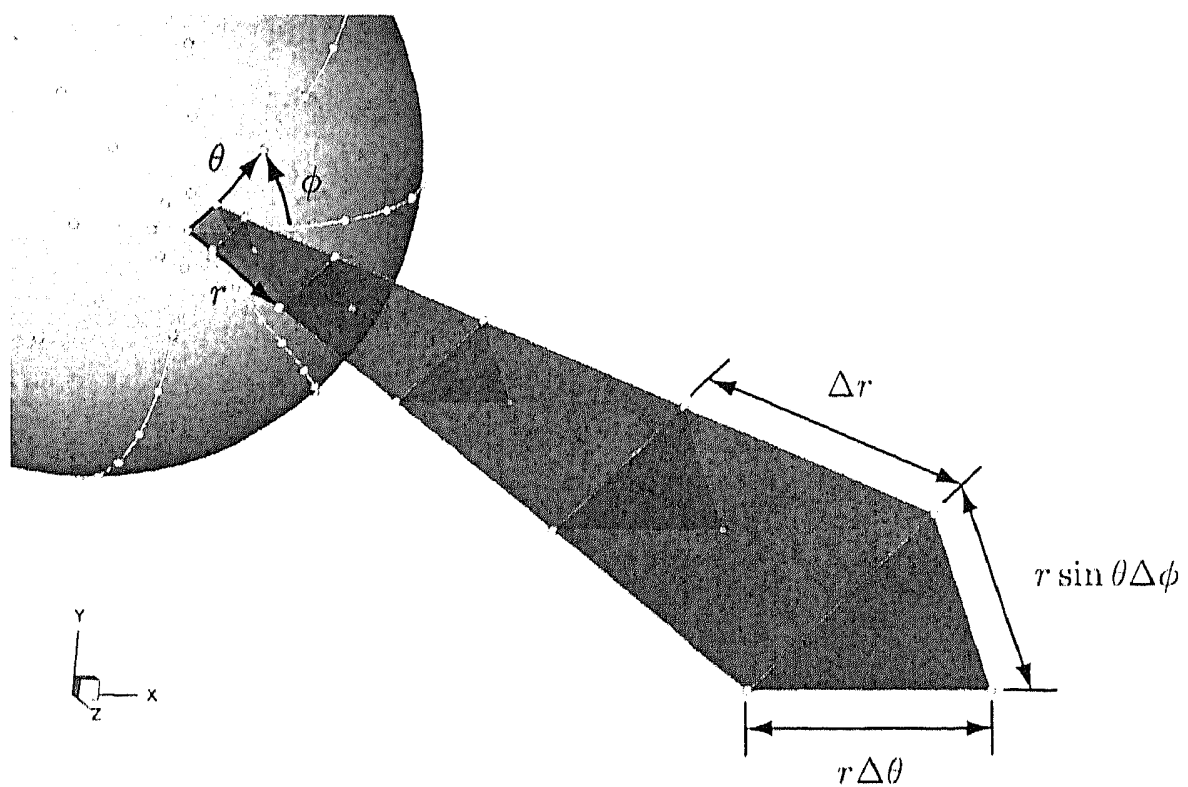

Figure 2.4: Schematic representation of the collocation point spacing in the vicinity of the sphere poles.

immthat diffusive terms in the momentum equations. Details regarding the treatment, of the diflusive terms are provided in $\$ 2.7$. The time-step size is then restricted by the Courant lricelrichs Levy (CFL) condition, which in spherical coordinates is given by

$$
C F L=\left(\frac{u_{r}}{\Delta r}+\frac{u_{\theta}}{r \Delta \theta}+\frac{u_{\phi}}{r \sin \theta \Delta \phi}\right) \Delta l^{*}
$$

where $\Delta r, r \Delta \theta$ and $r \sin \theta \Delta \phi$ are the spacing between collocation points in the radial, tamgential and azimuthal direction, respectively.

$\Lambda$ commen method to alleviate the 'pole problem' is to lower the resolution near the poles. In the case of a spectral expansion, this is easily achioved by filtering the highfrefunency liourier components (see, for example, Umscheid \& Sankar-Rao) (1970) and Fornberg (1997)). Since the grid spacing decreases both as we approach the poles and alse) as we approach the sphere surface, the filter should be designed to be a function of both $r$ and $\theta$. The filter must also be sufficiently smooth to preserve the spectral convergence of the Fourior expansions. At the poles, regularity constraints dictate that the azimuthal Fourier expansions for a scalar ficld should comprise only mode $k=0$, while those for a vector should contain only the $k=1(\cos \theta)$ and $k=-1(\sin \theta)$ 
modes. $\dagger$ Physically, the $k= \pm 1$ modes are the most unstable modes. They initiate the first bifurcation of the flow (Natarajan \& Acrivos, 1993) and must be retained over the entire computational domain. In light of these requirements, a filter that has a suitable form is

$$
g_{\phi}(r, \theta, k)=1-\exp \left(-\xi(k) Y^{\varphi(k)}\right)
$$

where $k$ is the azimuthal wavenumber and $Y=r \sin \theta$ is the distance measured normal to the polar axis ( $z$-axis). $\xi(k)$ and $\varphi(k)$ are functions only of $k$ and are solved subject to the conditions

$$
g_{\phi}=\frac{1}{k} \text { at } Y=Y_{\min } \text { and } g_{\phi}=0.9 \text { at } Y=k: Y_{\min }
$$

Here $Y_{\text {min }}$ is the collocation point at the sphere surface, closest, to the sphere pole. Recall that the collocation points in the tangential direction are staggered to avoid the singularitics at the poles. Figure 2.5 presents $g_{\phi}$ versus $Y$ for increasing azimuthal wavenumber, $k$. The filter approaches unity exponentially, such that, it, is active only in a very small region near the poles of the sphere. The filtered region increases gradually as the azimuthal wavenumber increases. This filter is applied to the intermediate velocity fiold, before the pressure correction step.

\subsection{Spatial differentiation}

Differentiation in the radial and tangential directions is mest conveniently performed in physical space, by multiplying the discrete vector variable of interest with an appropriate differentiation operator matrix. For the azimuthal direction, the periotic nature of the primitive variables allows azimuthal derivatives to be calculated in wavemumber space (or Fourier space). The operator matrices constructed for differentiation in the

$\dagger$ At the poles, a scalar must be single valued, which dictates that only the mon azimuthal mode can exist there. In spherical coordinates a vector may be multi-valued at the pole, while a cartesian vector is single valued. The transformation from a cartesian to spherical coordinale system introchees a mode 1 variation in the vector components. Hence, a vector in spherical coordinates comprises modes $k= \pm 1$ at the poles. 


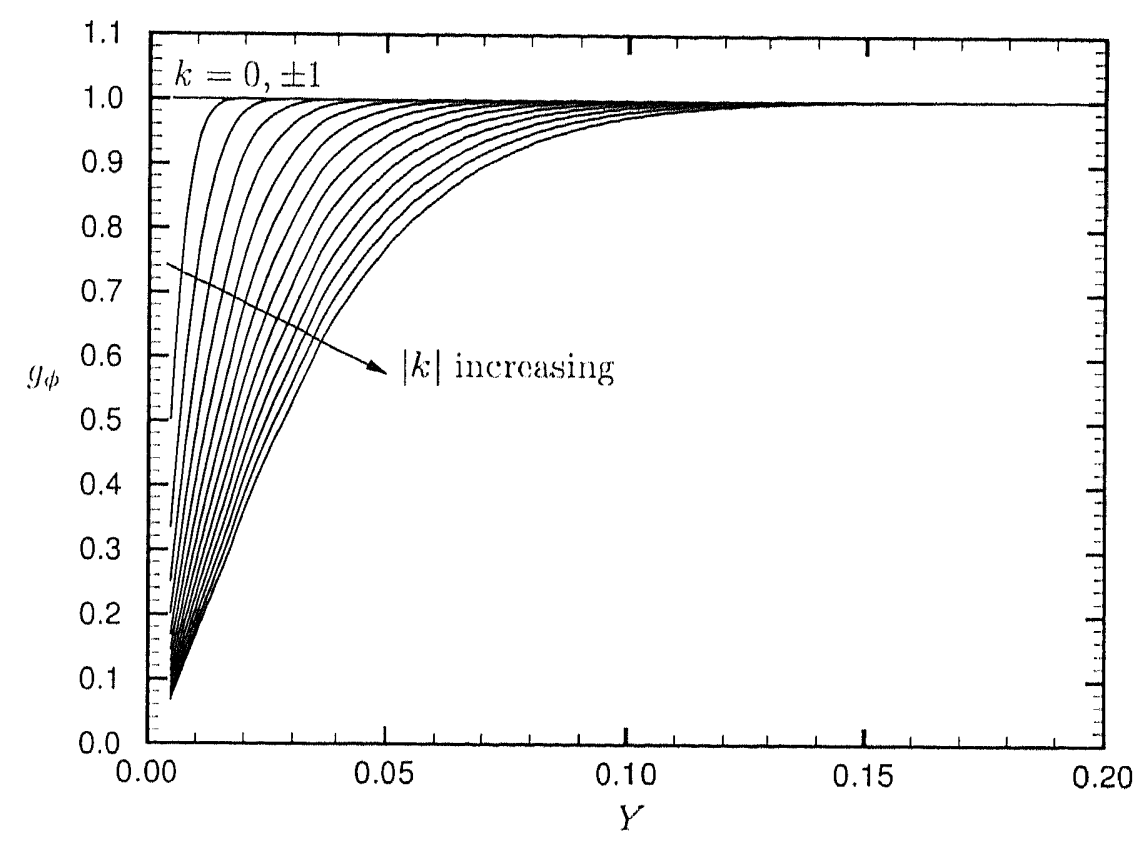

Figure 2.5: Pole filter, $y_{\phi}$, versus collocation point spacing, $Y$, with azimnthal wavenumber as a parameter. Filtering is confined to a very small region elose to the polcs (small $Y$ ). Filtoring increases with increasing wavemumber, while no filtering is applicel to $k:=0$, 土1.

radial and tangential directions are described below. Differentiation in the azimuthal direction is not discussed, as it is a standard method that is well described in the open litcrature (sece, for cxample, Moin (20)1), Canuto el al. (1988) and Got,licb \& Orszag $(1997))$.

\subsubsection{Radial derivatives}

In physical space, differentiation of the discrete variable, $f\left(x_{j}\right)$, can be conveniently represcrented in matrix form as

$$
\left(\frac{\partial f}{\partial x}\right)_{l}=\sum_{j=1}^{N_{r}} \mathcal{D}_{l j}^{1} f\left(x_{j}\right), \quad l=1,2, \ldots, N_{r} .
$$

For the Gauss Labalto distribution of collocation points given by equation (2.16), the 
elements of the Chebyshev collocation derivative matrix arc

$$
\mathcal{D}_{l j}^{1}= \begin{cases}\frac{1}{2} \frac{(-1)^{l+j}}{\sin \frac{\pi}{2 N_{r}-1(l+j-2)} \sin \frac{\pi}{2 N_{r}-1}(l-j)} & l \neq j \\ \frac{1}{2} \frac{\cos \left(\frac{\pi}{N_{r}-1} l\right)}{\sin ^{2}\left(\frac{\pi}{N_{r}-1} l\right)} & l=j, \quad i \neq 1, N_{r} \\ -\frac{2\left(N_{r}-1\right)^{2}+1}{6} & l=j=1 \\ \frac{2\left(N_{r}-1\right)^{2}+1}{6} & l=j=N_{r} .\end{cases}
$$

Don \& Solomonoff (1995) showed that for a machine precision of $\epsilon$, the entrics in the top half of $\mathcal{D}_{l j}^{1}$ are more accurately represented, than these in the lower half. To improve the ovcrall accuracy of $\mathcal{D}_{l j}^{1}$, only the more accurate top half of $\mathcal{D}_{l j}^{1}$ is computed, while the bottom half is obtained using the property

$$
\mathcal{D}_{l j}^{1}=-\mathcal{D}_{N_{r}-l, N_{r}-j}^{1} \quad i=\frac{N_{r}}{2}+1, \ldots, N_{r}
$$

This 'flipping' of the differentiation matrix reduces the overall round-off error of the matrix-vector multiplication given by equation $(2.30)$ from $\mathcal{O}\left(N_{r}^{3} \mathrm{e}\right)$ to $\mathcal{O}\left(N_{r}^{2} \mathrm{e}\right)$.

By introducing the transformation vector, $\partial x / \partial r$, the derivalives of $f$, in the physical domain, can be obtained via the chain rule. The first derivative, for example, is given as

$$
\begin{aligned}
\left(\frac{\partial f}{\partial r}\right)_{l} & =\left(\frac{\partial x}{\partial r} \frac{\partial f}{\partial x}\right)_{l} \\
& =\left(\frac{\partial x}{\partial r}\right)_{l} \sum_{j=1}^{N_{r}} \mathcal{D}_{l j}^{l} f\left(x_{j}\right) \\
& =\sum_{j=1}^{N_{r}}\left(\frac{\partial x}{\partial r}\right)_{l} \mathcal{D}_{l j}^{1} f\left(x_{j}\right) \\
& =\sum_{j=1}^{N_{r}} \mathbb{D}_{l j}^{1} f\left(x_{j}\right),
\end{aligned}
$$

where $\mathbb{D}_{l j}^{1}$ is defined as a physical space difforentiation matrix. To construct physicalspace derivative matrices of order $n, \mathbb{D}_{l j}^{1}$ is multiplied with itsclf, $n-1$ times. 


\subsubsection{Tangential derivatives}

The physical-space Fourier cosine and Fourier sine derivative operator matrices may be expressed in terms of the components of the general Fourier derivative operator matrix, as described in Appendix F of Boyd (1989). For $2 N_{0}$ degrees of freedorn, the first-order Fourier derivative matrix may be expressed as

$$
\mathcal{F}_{l j}^{1}= \begin{cases}\frac{1}{2}(-1)^{l-j} \cot \frac{\pi(l-j)}{2 N_{\theta}} & l \neq j \\ 0 & l=j,\end{cases}
$$

and the second-order Fourier derivative matrix as

$$
\mathcal{F}_{l j}^{2}= \begin{cases}\frac{1}{2}(-1)^{l-j+1} \sin ^{2} \frac{\pi(l-j)}{2 N_{\theta}} & l \neq j \\ -\frac{2+N_{0}^{2}}{12} & l=j .\end{cases}
$$

The first-order Fourier cosine derivative matrix, for $N_{0}$ degrees of freedom, may be expressed in terms of the elements of equation (2.34) as

$$
\mathcal{C}_{l j}^{1}=\mathcal{F}_{l j}^{1}+\mathcal{F}_{l, 2 N_{0}+1-j}^{1} \quad l, j=1,2, \ldots, N_{0}
$$

and the first-order fourier sine derivative as

$$
\mathcal{S}_{l j}^{1}=\mathcal{F}_{l j}^{1}-\mathcal{F}_{l, 2 N_{\theta}+1-j}^{1} \quad l, j=1,2, \ldots, N_{0}
$$

Higher order clerivative matrices can not, be constructed by multiplying the first-order derivative operator with itself, as is the case for the Chebyshev operator matrices. For example, the second-order Fourior cosine derivalive matrix is $\mathcal{C}^{2}=\mathcal{S}^{1} \mathcal{C}^{1}$, while the second-order fourier sine derivative matrix is $\mathcal{S}^{2}=\mathcal{C}^{1} \mathcal{S}^{1}$. Nlternatively, the secondorder derivative matrices may be constructed from the elements of equation (2.35), that is

$$
\mathcal{C}_{l j}^{2}=\mathcal{F}_{l j}^{2}+\mathcal{F}_{l, 2 N_{\theta}+1-j}^{2} \quad l, j=1,2, \ldots, N_{\theta}
$$

and

$$
S_{l j}^{2}=\mathcal{F}_{l j}^{2}-\mathcal{F}_{l, 2 N_{\theta}+1-j}^{2} \quad l, j=1,2, \ldots, N_{\theta}
$$


Mapping the above operator matrices to the physical domain is achieved in an analogous way to that described for the Chebyshev derivative matrices. In this case, the mapping is applied to the Fourier derivatives before the decomposition into the cosine and sine derivative matrices. As indicated by the spectral expansions given by cquations (2.21) to (2.24), whether a cosine or sine opcrator is appropriate depends on whether the function is a scalar or a vector. Differentiation of a function with definite parity reverses the parity if differentiation is repeated an odd number of times, and leaves the parity unchanged if the function is differentiated an even number of times.

\subsection{Temporal discretization}

The governing equations are temporally discretized using a seconcl-order variation of the fraction-step (or projection) method pioncered by Chorin (1968). In essence, this involves the solution of an approximation to the momentum equations for an intermediate velocity field $u^{*}$, without regard to the incompressibility constraint, followed by a projection of $u^{\star}$ onto a divergenec free space, $u^{n+1}$. Brown el al. (20)1) noted that nearly all numerical methods for solving the incompressible Navier Stokes equations, in terms of the primitive variables, use a fractional-step approach. This appears to be a result of their numerical efficiency (the deternining factor for its chesece in this study) and the case with which the methorl may be implemented, since existing solvers for advection-diffusion and Poissone ecpuations can be simply applied to the solution of the Navier Stokes ecplations.

This simplicity is deceptive and since their inception there has beren ongoing debate ancl confusion regarding the most appropriate choice of boundary conclitions for the intermediate velocity variables and for the pressure field. The issues involved in choosing boundary conditions that are consistent with the governing equations are cliseussed by Orszag et al. (1986), Gresho \& Sani (1987), Temam (1991), Brown et al. (2001) and Petcrsson (2001), among others. An inappropriatc choice of boundary conditions may result in numerical boundary layers in the primitive variables and this has lead to cr- 
roneous conclusions about the global accuracy of the fractional-step methods. Scveral commonly used variations of the fractional-step method are considered in this study. These are second-order accurate for velocity and first or second-order accurate for pressure. Although the accuracy of these schemes has been the subject of a large number of studies, the results are often conflicting. Their accuracy is therefore recvaluated by numerical experiment in chapter 3 for the case of the uniform flow past, a sphere.

To arrive at the second-order time-discrete form of equations (2.1) and (2.2), the non-linear convective terms are treated using an explicit second-order Adams Bashforth discretization, and the lincar diffusive terms using a second-order, semi-implicit Crank Nicolson discretization, to improve stability. This leads to

$$
\frac{u^{n+1}-u^{n}}{\Delta t}+\frac{3}{2}\{u \cdot \nabla u\}^{n}-\frac{1}{2}\{u \cdot \nabla u\}^{n-1}=-\nabla P^{n+\frac{1}{2}}+\frac{\nu}{2} \nabla^{2}\left\{u^{n+1}+u^{n}\right\}
$$

andel

$$
\nabla \cdot u^{n+1}=0
$$

Note that in ecquation (2.40) the pressure gradient term $\left(\nabla P^{n+\frac{1}{2}}\right)$ is time-centerex to be consistent with the Crank Nicolson treatment of the linear terms. These equations are solved subject, to Dirichlet velocity boundary conditions of the form

$$
\left.u^{n+1}\right|_{\partial \Gamma^{\prime}}=u_{b}
$$

Equations (2.40) to (2.42) are cumbersome to solve due to the coupling between the momentum ecputions, through the pressure term. Using the fractional-step method, the pressure term is chroped and approximations to the momentum equations are solved for the anxiliary velocity field, $u^{\star}$. This is the so-called advection-diffusion step) and has the form

$$
\frac{u^{\star}-u^{n}}{\Delta l}+\frac{3}{2}\{u \cdot \nabla u\}^{n}-\frac{1}{2}\{u \cdot \nabla u\}^{n-1}=-\nabla q+\frac{\nu}{2} \nabla^{2}\left\{u^{\star}+u^{n}\right\}
$$

Here $q$ is some sort of approximation to the pressure and its precise form depends on the variation of the fractional-step method used. The choice of $q$ affects the degree with 
which the intermediate and projected velocity ficlds differ and therefore influences the most appropriate choice of boundary conditions for the intermediatc velocity ficld.

The advection-diffusion step is followed by the pressure correction step

$$
\frac{u^{n+1}-u^{*}}{\Delta t}=-\nabla \Phi^{n+1}
$$

where $\Phi$ is a scalar variable that is related to the pressure ficld. By taking the divergence of equation (2.44) and applying the incompressibility constraint (2.41), a Poisson equation for $\Phi$ is obtained as

$$
\nabla^{2} \Phi^{n+1}=\frac{\nabla \cdot u^{\star}}{\Delta t}
$$

This equation is solved implicitly for $\Phi^{n+1}$, to ensure that the incompressibility constraint is satisfied to machine accuracy, at cvery time step. The solution of ecuation (2.45) requires a boundary condition for $\Phi^{n+1}$. It is common to use a homogeneous boundary condition that is consistent with the pressure correction given by equation (2.44), that is

$$
\left.\widehat{n} \cdot \nabla \Phi^{n+1}\right|_{\partial \Gamma^{\prime}}=0
$$

For a spherical coordinate system, this reduces to

$$
\left.\frac{\partial \Phi^{n+1}}{\partial r}\right|_{\partial r}=0
$$

The effect of this non-physical boundary condition is felt as a mumerical bounclary layer that extends several grid points away from the bonnlaries an example of this is illustrated in chapter 3. Once $\Phi^{n+1}$ is computed, equation $(2.44)$ can be rearranged (o) obtain the velocity field at the full time step

$$
u^{n+1}=\boldsymbol{u}^{\star}-\Delta t \nabla \Phi^{n+1} .
$$

Finally the pressure field is updated. The form of this update depends on the variation of the fractional-step used. In general it is given as

$$
P^{n+\frac{1}{2}}=q+\mathbb{L}\left(\Phi^{n+1}\right)
$$


where $\mathbb{L}$ is a lincar operator that relates $P^{n+\frac{1}{2}}, q$ and $\Phi$.

The boundary conditions for the intermediate velocity ficld are chosen to be consistent with equation (2.44) in order to preserve the accuracy of the scheme near the boundary, that is

$$
\left.\boldsymbol{u}^{\star}\right|_{\partial \Gamma}=\left.\left(\boldsymbol{u}^{n+1}+\Delta t \nabla \Phi^{n+1}\right)\right|_{\partial \Gamma}
$$

For the wall-normal velocity, cquation (2.44) together with equation (2.46) imply

$$
\widehat{n} \cdot u^{\star}=\widehat{n} \cdot u^{n+1}=0
$$

For the langential velocity components, $\widehat{\tau} \cdot \nabla \Phi^{n+1}$ is unknown prior to the calculation of $u^{\star}$ and must be approximated. If $u^{\star}$ differs significantly from $u^{n+1}$, the cstimate for $\nabla \Phi^{n+1}$ must be at least second-order accurate, in order to preserve the overall accuracy of the scheme. Strectt \& Hussaini (1991) expanded $\nabla \Phi^{n+1}$ in a Taylor series aboul $l=t^{n}$, as follows

$$
\nabla \Phi^{n+1}=\nabla \Phi^{n}+\Delta l \frac{\partial \nabla \Phi^{n}}{\partial t}+\left(\Delta l^{2}\right)
$$

then approximating $\partial \nabla \Phi^{n} / \partial t$ using a first-order backward difforence

$$
\frac{\partial \nabla \Phi^{n}}{\partial t}=\frac{\nabla \Phi^{n}-\nabla \Phi^{n-1}}{\Delta t}+\mathcal{O}(\Delta l)
$$

ccpution (2.52) becomes

$$
\nabla \Phi^{n+1}=2 \nabla \Phi^{n}-\nabla \Phi^{n \cdots 1}+\mathcal{O}\left(\Delta l^{2}\right)
$$

Finally, the wall-parallel boundary conditions for the intermediate variables become

$$
\hat{\tau} \cdot u^{*}=\hat{\tau} \cdot\left\{u^{n+1}+\Delta l\left(2 \nabla \Phi^{n}-\nabla \Phi^{n-1}\right)\right\}+\mathcal{O}\left(\Delta l^{3}\right)
$$

which results in a slip velocity at the full time step, ' $n+1$ ', of $\mathcal{O}\left(\Delta t^{3}\right)$.

Several fractional-step methods, which vary by their choice of $q$ and $\mathbb{L}$, are described in the following sections. 


\subsubsection{Fractional-step method I (Fsm I)}

A commonly used variation of the fractional-step method takes $q=0$ and $\mathbb{L}=I$.

The choice of $\Phi=P^{n+\frac{1}{2}}$ results in a numerical boundary layer in the pressure field. This is a well-known artifact and has been reported to degrade the overall temporal accuracy of the pressure field to first-order (sec, for example, Brown el al. (2001)). A sccond-order accurate velocity field may be recovered using equation (2.55) as the boundary condition for the wall-parallel velocities.

\subsubsection{Fractional-step method II (Fsm II)}

The variation introduced by Kim \& Moin (1985) takes $q=0$ and the linear operator $\mathbb{L}$ is chosen such that the a second-order accurate pressure field is recovered up to the boundaries. By adding together cquation (2.43) and equation (2.44), the second-order discretization of the momentum equations (2.40) are recovered only if

$$
P^{n+\frac{1}{2}}=\Phi^{n+1}-\frac{\nu \Delta l}{2} \nabla^{2} \Phi^{n+1}
$$

Therefore

$$
\mathbb{L}=I-\frac{\nu \Delta t}{2} \nabla^{2} \Phi^{n+1}
$$

As noted by Perot (1993), the above correction assimmes that, the granlient ( $\nabla$ ) and the Lelplacian $\left(\nabla^{2}\right)$ operators commute, that is, $\nabla \nabla^{2}=\nabla^{2} \nabla$. This is true for the continuous operators and it is also true for the spectral oporators employed in this study. The second term in equation (2.56) is known as the crror absorbing torm and is important for computing the correct pressure gradient near bomedaries. Brown et al. (2001) noted that from equation (2.45), equation (2.56) may also be written as

$$
P^{n+\frac{1}{2}}=\Phi^{n+1}-\frac{\nu}{2} \nabla \cdot u^{\star}
$$

This is true at all interior points, but not at the boundaries where the correction is most important. Equation (2.45) is solved only at interior points, subject to the boundary condition (2.46), without regard to the behavior of $\nabla \cdot u^{\star}$ at the boundaries. 
Although more computationally expensive, we opt to use $\nabla^{2} \Phi$ in preference to $\nabla \cdot u^{*}$, since the later is, in gencral, not smooth near boundaries (Brown et al., 2001). This is particularly important when the fractional-step method of Boll et al. (1989) is cmployed, because the gradient of pressure appears in the advection-diffusion step.

The choice of $q=0$ results in $u^{\star}$ and $u^{n+1}$ differing by $\mathcal{O}(\Delta t)$. In order to recover a second-order accurate velocity field, the boundary condition given by equation (2.55) must be employed. The choice of $q=0$ also means that the pressure correction does not feed back into the advection-diffusion equation. It is purely a post-processing step and need only be performed when the pressure field is required.

\subsubsection{Fractional-step method III (Fsm III)}

Bell et al. (1989) made the choice $q=P^{n-\frac{1}{2}}$ and $\mathbb{L}=\nabla$, such that

$$
\nabla P^{n+\frac{1}{2}}=\nabla P^{n-\frac{1}{2}}+\nabla \Phi^{n+1}
$$

This upedate procedure produces a numerical boundary layer in the pressure and eonsecuently produces a first-order rate of convergence for the pressire field. Using $q=P^{n} \cdot \frac{1}{2}$ leads to a greater coupling between the advection-diffusion and pressure correction st.cps. $\Lambda$ s a result, $u^{\star}$ and $u^{n+1}$ differ by at most $\mathcal{O}\left(\Delta t^{2}\right)$, which moans that it is sullicient to set $\left.\boldsymbol{u}^{\star}\right|_{\partial \Gamma}=\boldsymbol{u}_{b}$ for all velocity components and still recover a scconclorder accurate velocity field. Brown et al. (2001) suggested that, for a more acecurate pressure-gradient, at the bonndary, the error absorbing term in equation (2.56) should also be included as

$$
\nabla P^{n+\frac{1}{2}}=\nabla P^{n-\frac{1}{2}}+\nabla \Phi^{n+1}-\frac{\nu \Delta l}{2} \nabla \nabla^{2} \Phi^{n+1}
$$

To the authors knowledge, this update procedure has never been antlyzed. In subsecplent discussions, Fsm III will refer to the pressure correction defined by equation (2.60). 


\subsection{Velocity boundary conditions}

The boundary conditions for $\boldsymbol{u}^{n+1}$ at the outer boundary (the far-ficld boundary) and inner boundary (the sphere surface) of the computational domain are described, in turn, below.

\subsubsection{Far-field boundary conditions}

Spatial discretizations using spectral methods are notoriously sensitive to the imposed boundary conditions due to their global nature. The boundary conditions at the outer boundary of the computational domain have been derived with this in mind, whilst also bcing consistent with the fractional-step temporal discretization.

The far-ficld boundary is divided at the equatorial plane into an inflow region $(\theta \geq \pi)$ and outflow region $(\theta<\pi)$. Over the inflow region, a Dirichlet bounclary condition prescribes the inflow as the potential flow solution around a stationary sphere. This boundary condition does not allow for the displacement effects clue to the viscous flow around the sphere, however, for a sufficiently large computational domain, the displacoment effects become relatively small. Over the ontflow bounclary, the wake should be convected out of the computational domain withont, reflection and with minimal distortion. With spectral based methods, it is common to cmploy non-reflective boundary conditions, which render the governing couations parabolic near the outflow bomulary. In practice, this involves smoothly attemating the radial dillusive torms in the momentum equations to zero, through multiplication by a suitable filtor function. Here, the filter function devised by Mithal (1995) is used and has the form

$$
g(r, \theta)=1-\operatorname{cxp}\left[-\zeta\left\{\left(\frac{r-r_{1}}{r_{1}-r_{n r}}\right)^{\prime \prime \prime}+\left(\frac{\theta}{\pi}\right)\right\}^{\prime / 2}\right] .
$$

For the problem under investigation, the optimal parameters in this ecplation were found by numerical experiment to be $\zeta=40.0, \eta_{1}=3.23$ and $\eta_{2}=5.10$. Figure 2.6 presents $g(r, \theta)$ and its first derivatives with respect to both $r$ and $\theta$. Filtering is limited to the outflow region only, where $g(r, \theta)$ smoothly goes 1,0 zero in both the radial and tangential directions. The first derivatives of $g(r, \theta)$ are also smooth and are 


\subsection{VELOCITY BOUNDARY CONDITIONS}

non-zcro only near the outflow boundary. Mittal (1995) and Mittal (1999b) rigorously tested this filter function and have shown that it allows vortical disturbances to exit the computational domain in a smooth mannor without significant reflection. The performance of the filter is also demonstrated in chapter 3.

The boundary condition applied over the outflow boundary is of a convective lype. In $\$ 2.7$ the advection-diffusion equations for the intermediate velocity ficld, $u^{\star}$, are shown to reduce to a set of one-dimensional Helmholtz equations of the form

$$
\frac{1}{2} \sin ^{2} \theta g(r, \theta) \frac{\partial}{\partial r}\left(r^{2} \frac{\partial u^{\star}}{\partial r}\right)-\frac{r^{2} \sin ^{2} \theta R e}{\Delta t} u^{\star}-\frac{1}{2} \frac{\partial^{2} u^{\star}}{\partial \phi^{2}}=R \cdot H S^{\star}
$$

where the $R H S^{\star}$ comprises all terms in the advection-diffusion equation that are treated explicitly. At the outflow boundary, $g(r, \theta)=0$ and equation (2.62) may be rearranged to provide the following convective boundary condition for the intermediate velocity

$$
u^{\star}=\frac{R H S^{\star}}{\left(\frac{1}{2} \frac{\partial^{2}}{\partial \phi^{2}}-\frac{r^{2} \sin ^{2} \theta R e}{\Delta t}\right)}
$$

\subsubsection{Surface boundary conditions}

$\Lambda$ the sphere surface, the no-slip and no-penctration boundary conditions are prescribed. For a rotating sphere, the surface velocity distribution is computed by laking the cross product of the angular velocity vector, $\Omega$, and the surface position vector, $\mathrm{r}=(d / 2) \widehat{e}_{r}$. For a sphere rotating about the $x$-axis, with a constant angular speed, $\Omega$, the angular velocity vector is

$$
\Omega=\Omega\left(\sin \theta \cos \phi \widehat{\boldsymbol{e}}_{\boldsymbol{r}}-\cos \theta \cos \phi \widehat{\boldsymbol{e}}_{\theta}+\sin \phi \widehat{\boldsymbol{e}}_{\phi}\right)
$$

and the velocity at the sphere surface is

$$
\left.\boldsymbol{u}\right|_{\partial \Gamma}=\Omega \times r=-\frac{\Omega d}{2}\left(\sin \phi \widehat{\boldsymbol{e}}_{\boldsymbol{\theta}}-\cos \theta \cos \phi \widehat{\boldsymbol{e}}_{\phi}\right) .
$$

For a sphere undergoing rotary oscillation, the angular volocity varies sinusoidally and $\Omega$ in the above expression should be replaced by $\Omega_{0} \sin \left(2 \pi S t_{f} t^{*}\right)$, where $S t_{f}$ is the Strouhal number of forcing and $\Omega_{0}$ is rotation rate amplitude. 


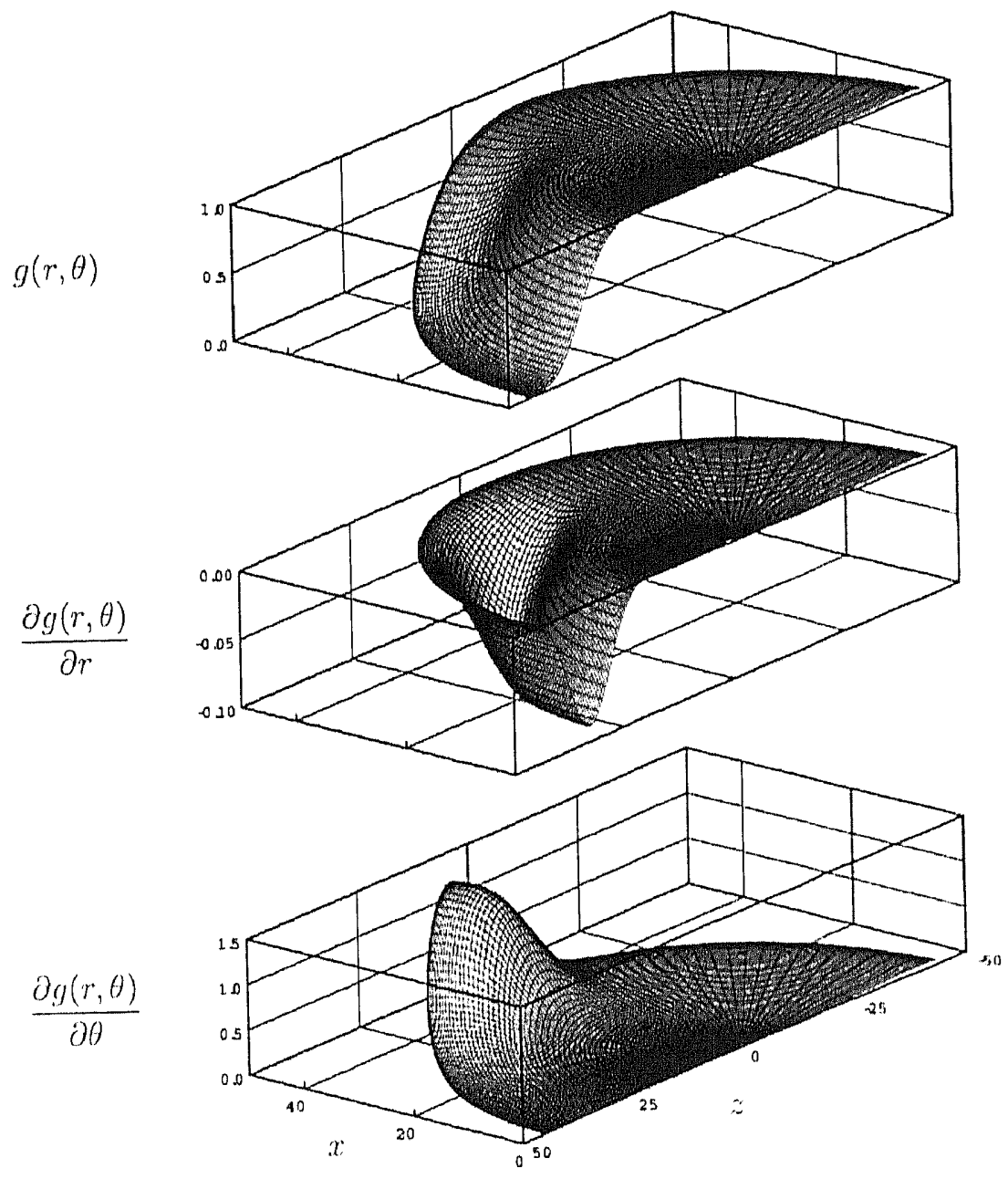

Figure 2.6: Non-reflective filter function, $g(r, \theta)$, and its first derivatives with resperet to

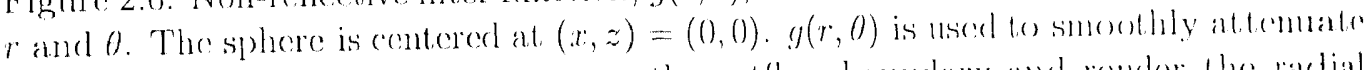
the raclial diffusive terms to zero near the ontellow bonudary and render the ractial

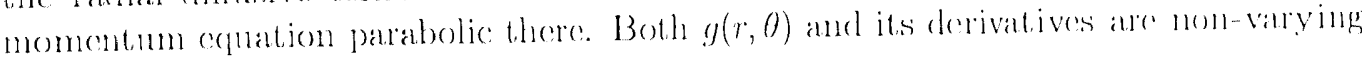
near the sphere.

\subsection{Initial conditions}

The primitive variables are initialized using a potential solution for the uniform flow past a stationary sphere. At $t^{*}=0$, the no-slip condition is applied impulsively lo the sphere surface. For a stationary sphere, these initial comlitions alone lead to a steady axisymmetric flowficld, even above the Reynolds number for which the wake: 
is known to become asymmetric. In physical experiments, the flow asymmetry is caused by instabilities that are amplified from small disturbances in the flow. These disturbances may be a result of frecstream turbulence or surface irregularities, for example. Disturbances of this nature are absent in numerical simulation. Hence, to break the flow axisymmetry it is necessary to apply an artificial perturbation in the carly stages of the simulation.

It is common to introduce random noise in the incoming flow, upstream of the: body. The drawback with such a perturbation, is that it is only folt in the vicinity of the sphere after a time of order $D_{0} / U_{\infty}$. Here, a perturbation is applied ath the sphere surface, comprising a mass conserving tangential slip velocity with azimuthal mode 1 variation, which is given by

$$
\left.u_{0}\right|_{i I^{2}}=-\Omega^{*} \sin (\phi) \sin \left[2 \pi\left(l^{*}-l_{s}^{*}\right) /\left(l_{f}^{*}-l_{s}^{*}\right)\right]
$$

where $\Omega^{*}=0.01$ and $l^{*}=l_{s}^{*}=0.05$ l.o $t^{*}=l_{f}^{*}=0.10$. A number of other perturbation profiles, amplitudes and durations were considered; no effect was observed in the solution over the paraneter range considered. For Reynolds mumbers below that, at which the regular bifurcation occurs (Re $<212$ ), the perturbation decalys and the flow returns te axisymmetry. For the rotating sphere simulations, the preseribed surface velocity is sullicient, to broak the flow axisymmetry.

\subsection{Solution algorithm}

'lhe numerical algorithm comprises two steps, an advection-diflusion stcp, followed by a pressure correction step. At the advection-diffusion step, the lincar terms are treated in a semi-implicit manner. In spherical coordinates the presence of non-constant scale factors results in a vector Laplacian that couples the monentum equations and makes implicit, treatment of the diffusion terms difficult. To alleviate this problem, only the first term in equations (2.7), (2.8) and (2.9) are treated implicitly, that, is, only the terms that comprise the scalar Laplacian. Furthermore, because the grid spacing in 
the tangential direction is significantly larger than that in the radial and azimuthal directions, it is also possible to treat the tangential component of the scalar Laplacian explicitly, without negatively impacting on the temporal stability of the solver. This leads to a set of Helmholtz equations of the form

$$
\frac{1}{2} g(r, \theta) \frac{1}{r^{2}} \frac{\partial}{\partial r}\left(r^{2} \frac{\partial \widehat{u}^{\star}}{\partial r}\right)-\frac{R e}{\Delta t} \widehat{u^{\star}}+\frac{1}{2} \frac{1}{r^{2} \sin ^{2} \theta} \frac{\partial^{2} \widehat{u^{\star}}}{\partial \phi^{2}}=(R H S)^{n},
$$

where $g(r, \theta)$ is the filter function used to render the momentum equations parabolic near the outflow boundary, and $(R H S)^{n}$ contains all the terms that are treated $\mathrm{ex}$ plicitly. By multiplying through by $r^{2} \sin ^{2} \theta$ and taking a Fourier transform in the $\phi$-direction, a set of variable coefficient onc-dimensional Helmholtz eculations are obtained, of the form

$$
\frac{1}{2} \sin ^{2} \theta g(r, \theta) \frac{\partial}{\partial r}\left(r^{2} \frac{\partial \widehat{u}^{\star}}{\partial r}\right)-\left(\frac{r^{2} \sin ^{2} \theta R e}{\Delta t}+\frac{1}{2} k^{2}\right){\widehat{u^{\star}}}^{2}=(\widehat{R H S})^{n},
$$

which can be solved independently for each azimuthal wavenumber, $k$ :

Once the intermediate velocity field is computed, it is prejected onter a divergenec frec fiold via a pressure correction step. This requires the solution of the Poisson cqualion

$$
\nabla^{2} \Phi=\frac{1}{r^{2}} \frac{\partial}{\partial r}\left(r^{2} \frac{\partial \Phi}{\partial r}\right)+\frac{1}{r^{2} \sin \theta} \frac{\partial}{\partial \theta}\left(\sin \theta \frac{\partial \Phi}{\partial \theta}\right)+\frac{1}{\left.r^{2} \sin ^{2} \theta\right)} \frac{\left.\partial^{2} \phi\right)}{\partial \phi r^{2}}=\frac{\nabla \cdot \boldsymbol{u}^{*}}{\Delta t} .
$$

By multiplying through by the common factor of $r^{2}$ and laking a fourier transform in the $\phi$-direction, the E'oisson requation takes the final form

$$
r^{2} \widehat{\nabla^{2} \Phi}=\frac{\partial}{\partial r}\left(r^{2} \frac{\partial \widehat{\Phi}}{\partial r}\right)+\frac{1}{\sin \theta} \frac{\partial}{\partial \theta}\left(\sin \theta \frac{\partial \widehat{\Phi}}{\partial \theta}\right)-\frac{k^{2} \widehat{\Phi}}{\sin ^{2} \theta}=r^{2} \frac{\widehat{\nabla \cdot u^{*}}}{\Delta t}
$$

Equation (2.70) is solved in a fully implicit manner to cusure that the incompressibility constraint is satisfied to machine precision. In the sturly of Mittal (1995) the source term in the above ecpuation was attenuated to zero at the outflow boundary to inhibit the reflection of pressure signals. This, however, introduces a non-zero divergence in the filtered region sinec the incompressibility condition is not enfored there. In this 
study a filter was not applicd to the source term, without a noticeable effect on the solution.

Both the Helmholtz equation (2.68) and the pressure Poisson equation (2.70), when represented in a fully discrete fashion, have the general form

$$
[\mathbb{A}][\widehat{u}]+[\widehat{u}][\mathbb{B}]^{T^{T}}-\beta[\widehat{u}]=[\widehat{\mathbb{R H I S}}] .
$$

In the above equation $[\mathbb{A}]$ is the diserete representation of the first term on the left hand side of the Helmholtz or Poisson equations. [ $\mathbb{B}$ ] is zero for the Helmholliz equation and it is the discrete representation of the second and third terms on the left hand side of the Poisson equation. $\beta$ represents the constant in the second term on the left hand side of the Helmholtz equation. [A] has dimensions of $N_{r} \times N_{r}$, while [ $[\mathbb{B}]$ has dimensions of $N_{\theta} \times N_{0}$. $[\widehat{u}]$ is the unknown velocity or pressure field matrix and has the dimensions of $N_{r} \times N_{0}$.

The global nature of the spectral representation is reflected in the discrete operators being dense matrices. In this casc, matrix inversion may he achiceved by cither an itcrative or direct method of solution. The author chose to employ the matrix rlaggonalization methos described by Canuto el al. (1988). Haidvodel \& Zang (1979) found this methes lo be an order of magnitude more efficient than the Alternating Direction Implicit $(\mathrm{ADI})$ itcrative scheme. However, it has the drawback of requiring a large amount of preprocessing to decompose the operator into eigenvalue and eigenvector matrices. As a result, the method is ideally suited to the solution of time-dependent problems, where the operators do not change at every time-step and the decomposition needs lo be done one when the problem is initialized. The solution prosedure, using the matrix diagonalization mothod, is illustrated bolow. First, the operator matrices are deromposed into their respective eigenvalue and eigenvector matrices, as follows

$$
[\mathbb{A}]=[\mathbb{M}]\left[\lambda_{r}\right][\mathbb{M}]^{-1} \text { and }[\mathbb{B}]=[\mathbb{N}]\left[\lambda_{\theta}\right][\mathbb{N}]^{-1}
$$

Here $[\mathbb{M}]$ and $[\mathbb{N}]$ are matrices formed by the eigenvectors of $\left[\nabla_{r}^{2}\right]$ and $\left[\nabla_{0}^{2}\right]$, respectively; while $\left[\lambda_{r}\right]$ and $\left[\lambda_{\theta}\right]$ are diagonal matrices of the eigenvalues of $\left[\nabla_{r}^{2}\right]$ and $\left[\nabla_{\theta}^{2}\right]$, respectively. 
Substituting equations (2.72) into equation (2.71) leads to

$$
[\mathbb{M}]\left[\lambda_{r}\right][\mathbb{M}]^{-1}[\widehat{u}]+[\widehat{u}][\mathbb{N}]\left[\lambda_{\theta}\right][\mathbb{N}]^{-1}-\beta[\widehat{u}]=[\widehat{\mathbb{R H} \mathbb{S}}] .
$$

Next, the matrices $[v]$ and $[s]$ are defined as

$$
[v]=[\mathbb{M}]^{-1}[\widehat{u}][\mathbb{N}] \text { and }[s]=[\mathbb{M}]^{-1}[\widehat{\mathbb{R H I S}}][\mathbb{N}]
$$

which, by pre-multiplying by $[\mathbb{M}]$ and post-multiplying by $[\mathbb{N}]^{-1}$, may also be written as

$$
[\widehat{u}]=[\mathbb{M}][\widehat{v}][\mathbb{N}]^{-1} \text { and }[\widehat{\mathbb{R} \mathbb{H} S}]=[\mathbb{M}][\widehat{s}][\mathbb{N}]^{-1}
$$

Substituting equations (2.75) into equation (2.73) and simplifying loads to

$$
\left[\lambda_{r}\right][v]+[v]\left[\lambda_{\theta}\right]-\beta[v]=[s] .
$$

Since $\left[\lambda_{r}\right]$ and $\left[\lambda_{\theta}\right]$ are diagonal matrices, the above expression simplifics to

$$
[v]\left[\lambda_{r}+\lambda_{\theta}-\beta\right]=[s]
$$

or

$$
[v]=\left[\lambda_{r}+\lambda_{0}-\beta\right]^{-1}[s]
$$

Here $\left[\lambda_{r}+\lambda_{0}-\beta\right]^{-1}$ is also a cliagonal matrix and therefore $[v]$ maty be recovered using only $N_{r} \times N_{\theta}$ operations, namely

$$
\begin{aligned}
{[v]_{i j}=\frac{[s]_{i j}}{\left[\lambda_{r}\right]_{i i}+\left[\lambda_{0}\right]_{j j}-\beta}, } & i=1,2, \ldots, N_{r} . \\
j & =1,2, \ldots, N_{0} .
\end{aligned}
$$

Once $[v]$ is obtained, $[u]$ may be recovered from equation (2.75)).

In the radial direction, boundary conditions of the Dirichlet type are recuired for the solution of the Helmholtz equations. These can be built into the formulation by modifying the first and last rows of the discretc operators and by modifying the corresponding terms in the right hand side of the equations to include the known boundary 
values. The application of Neumann pressure boundary conditions in the solution of the Poisson equation is achieved using the reduced matrix technique described by $\mathrm{Ku}$ et al. (1987). The application of a Neumann condition at both the inner and outer boundaries of the computational domain results in a pressure ficld that can only be evaluated up t,o an additive constant. This non-uniqueness is reflected in a zero cigenvalue in the decomposition of the discrete operator. The eigenvector associated with this eigenvalue is the constant mean pressure and is effectively set to zero, at cach time step, by assigning the inverse of the zero eigenvalue to zero. 


\section{Chapter 3}

\section{Code validation}

Before presenting the findings for a transverscly rotaling sphere, the accuracy of the solver is verified in a range of cases for which experimental and numerical data is available. The aim is to demonstrate the ability of the solver to accurately capture the flow physics over the parameter range of interest; namely, tRe $=2510300$ and non-dimensional rotation rates in the range $\Omega^{*}=0.00$ to 1.00 .

In $\$ 3.1$ the fractional-step methods introduced in chapter 2 are compared numerically and the method chosen for the remainder of the study is justified. An issue: of concern with the fractional-step method is that, in general, it fails to satisfy the incompressibility condition exactly at the boundarics. In $\$ 3.1 .1$ a method to reduce Whe surface divergenee is proposed and tested. For all simulations prosented in subse(fucut chapters, it has been verified that the results are independent of the spatial and temporal discretization. Examples to illustrate the measures taken to ascertain this are described in $§ 3.2$. To ensure disturbances are convected out of the computational domain without reflection, the outflow filter devised by Mittal (1995) is used. The performance of this outflow filter is demonstrated in $§ 3.3$.

Finally, in $\$ 3.4$ the solver is validated against available numerical and experimentaal results for a stationary and rotating sphere in the Reynolds number range of interest. 


\subsection{Evaluation of the fractional-step methods}

The three variations of the fractional-step method introduced in chapter 2 are compared for the case of a uniform flow past a stationary sphere at Re $=100$. This scenario is chosen because the flowfield remains steady and axisymmetric, thus simplifying data analysis. The analysis has been repeated for a stationary sphere at $R e=300$, but is not presented here becausc the trends are similar to that for $R e=100$.

Figure 3.1 presents the pressure component of drag, $C_{D_{r}}$, versus the non-dimensional time-step, $\Delta t^{*}$, for the various versions of fractional-step methods tested. The viscous component of drag is found to be insensitive to the choice of fractional-step method and therefore is not shown. As $\Delta t^{*}$ tends towards zero, the three mothods approach a single value, indicating that they are consistent. Both Fsm II and Fism III follow a similar profile and for all $\Delta t^{*}$ they show a smaller deviation from the limiting value of $C_{D_{p}}$ than for Fsm I. The cause of the difference in $C_{D_{P}}$, is apparent, from figure 3.2 , which presents the pressure, along a ray cmanating near the ecpuator of the sphere for $\Delta l^{*}=2.5 \times 10^{-4}$ and $5.0 \times 10^{-4}$. For Fsm 1 , the use of a homogeneous Nemmann boundary condition in the solution of the pressure field results in a mumerical houndary layer that affects the solution at several collocation points adjacent to the sphere: surface an observation which has also been made in a number of carlier studiess (sece, for cxample, Orszag el al. (1986)).

The crror absorbing term used in Fsm II and Fsm III works well to alleviate the mumerical boundary layer. Despite the inclusion of a lagging pressure gradiont term in the advection-diffusion step $\left(q=P^{n-1 / 2}\right)$, there is minimal differenee betwern the solutions produced by these two methods. Brown et al. (2001) noted that the error absorbing term plays a vital role in computing the correct pressure gradient at, the boundary, and allows the pressure to maintain the second order aceuracy up to the boundary. Without this correction, the pressure gradient may have first- or zerothorder accuracy at the boundary, and thus degrade the global accuracy of the scheme. 
Figure 3.3 presents the radial velocity profile, at the intermediate and full time-step, along a ray cmanating near the equator of the sphere. Fsm I and Fsm II differ only in a post-processing of the pressure ficld, while the velocity fields are identical. For this reason only Fsm I and III are shown. For Fsm III, the intermediate and corrected velocity profiles are indistinguishable. This arises because the lagging pressure gradient term is included in the advection-diffusion step $\left(q=P^{n-1 / 2}\right)$, causing $\Phi^{n+1}$ to remain small at all interior points. Although the corrected velocity profile is smooth, the wall-normal velocity gradient $(\partial u / \partial r)$ is non-zoro at the boundary. The divergenee in spherical coordinates may be written as

$$
\nabla \cdot u=\frac{\partial u_{r}}{\partial r}+\frac{2 u_{r}}{r}+\frac{1}{r \sin \theta} \frac{\partial\left(\sin \theta u_{0}\right)}{\partial \theta}+\frac{1}{r \sin \theta} \frac{\partial u_{\phi}}{\partial \phi} .
$$

$\Lambda$ the boundaries, the last three terms in this expression are idcentically zero and a non-zero value for $\partial u_{r} / \partial r$ leads to a non-zero divergenec. Because a single boundary condition is recuired in the solution of $u_{r}$, the zero-divergenee $\left(\partial u_{r} / \partial r=0\right)$ and zeropenctration $\left(u_{r}=0\right)$ conditions cannot be enforeed simultancously. To illustrate the importance of the boundary condition on $u^{\star}$, Brown et al. (2001) considered the "free bonndary condition,"

$$
\frac{\partial^{3} u_{r}^{*}}{\partial r^{3}}=0
$$

for a simple test problem, and showed that the divergence ean be significantly reduced. Based on the idea put forth by Brown et al., a modification to the fractional-step methed is propesed in $\$ 3.1 .1$, which significantly reduces the surface divergence while: alse satisfying the zero-penetration condition at the solid boundary.

It is apparent, from the limited analysis presented here that Fsm II and Fim III procluce sinilar results, while being more accurate than Fsm I in the recovery of pressure. F'sm II is less computationally demanding than Fsm III becausc only $\Phi^{n+1}$ needs to be computed at each time-step, while $P^{n+1}$ need only be computed when the corrected pressure is required. 


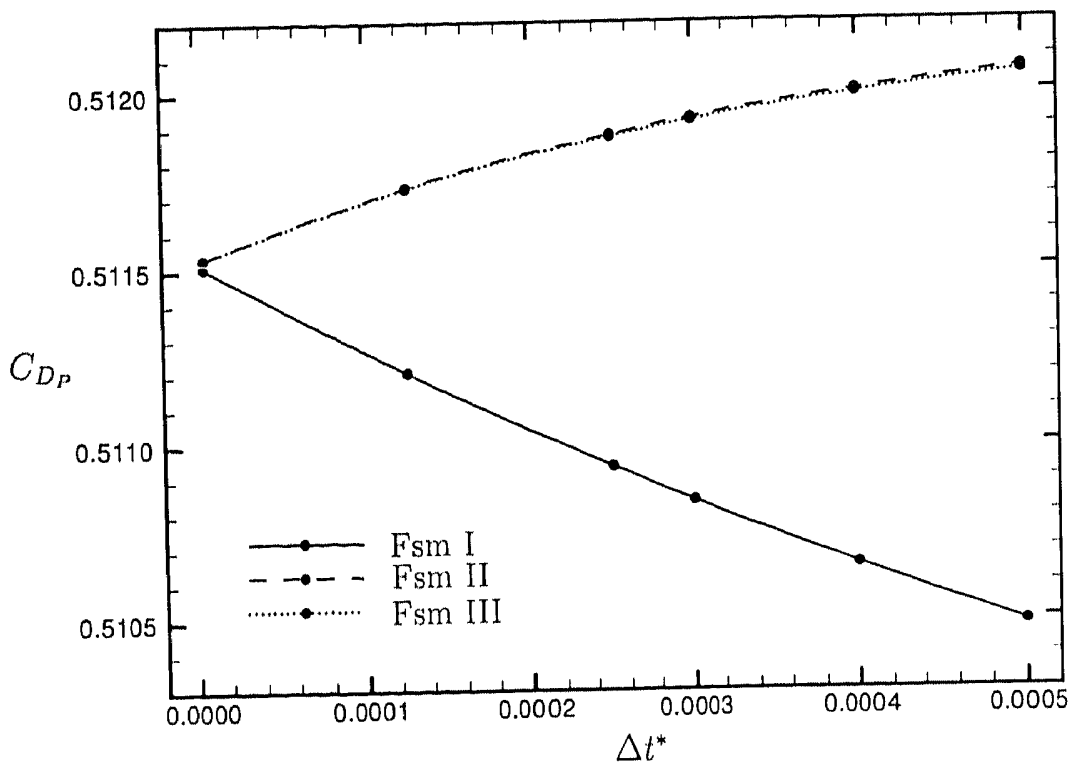

Figure 3.1: Pressure drag, $C_{D_{P}}$, versus $\Delta l^{*}$ for $R e=100$.

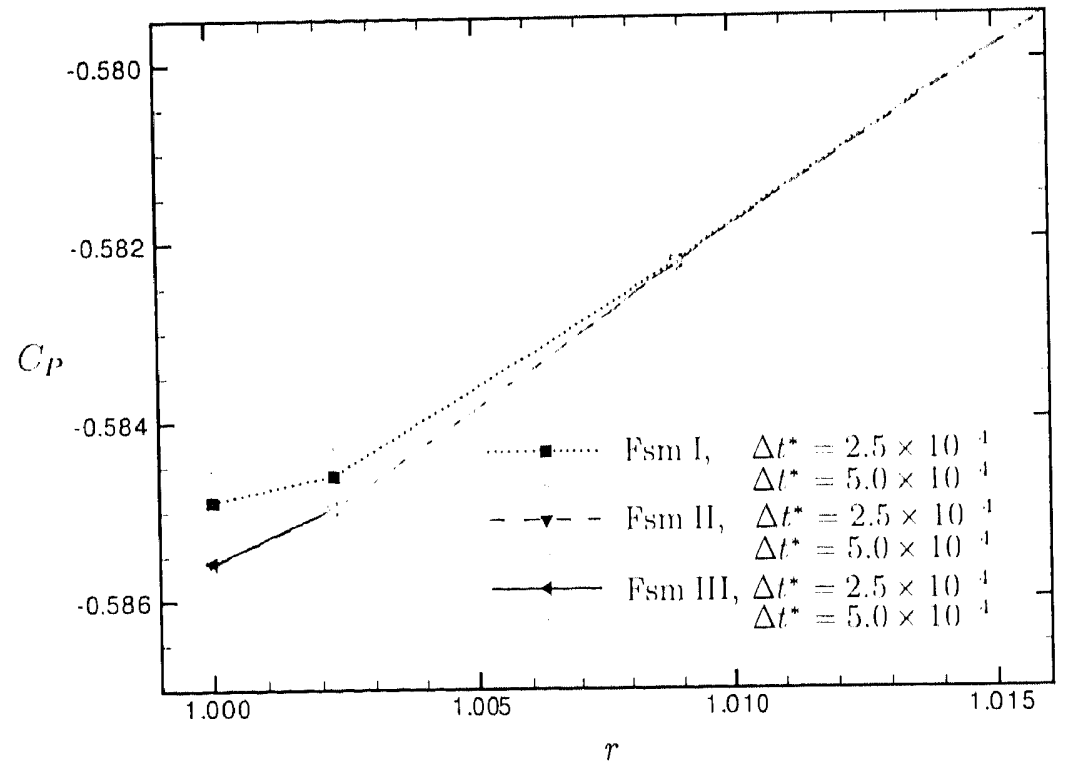

Figure 3.2: Pressure coefficient, $C_{P}$, along a ray near the equator of the sphere $(\theta=0.506 \pi)$ for $R e=100$. 


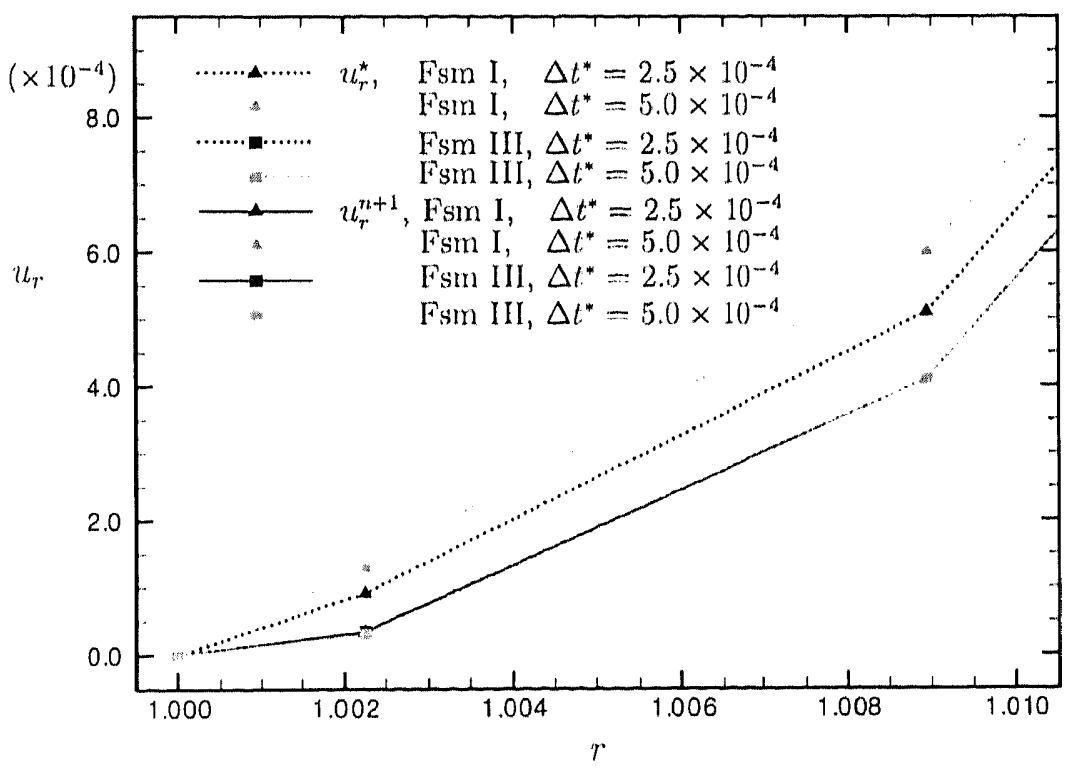

Fignre 3.3: Intermediate and projected radial velocity along a ray near the equator of the sphere $(\theta=0.506 \pi)$ for $R e=100$. 


\subsubsection{A Neumann boundary condition for the wall-normal velocity}

The most common implementation of the fractional-step method solves an advectiondiffusion equation subject to Dirichlet boundary conditions on $u^{\star}$. This is followed by the solution of a Poisson equation for pressure, subject to a homogencous Neumann boundary condition. The procedure ensures that the no-penetration condition is satisfied exactly at the full time-stcp, while incompressibility is enforeed only at interior points. At the boundaries, $\partial u_{r} / \partial r$ is generally non-zero and therefore the divergence is non-zero. Adjacent to no-slip boundaries, the two-step fractional-step mothod has been reported to produce a spurious numerical boundary layer in the wall-normal velocity and pressure field. This arises from the coupling of the pressure field to the velocity via the Poisson equation, with the divergence of the intermediate velocity field as the source term.

In an attempt to alleviate this problem, a modification to the numerical scheme is proposed here. To keep the wall-normal velocity smooth up to the boundary and reduce the surface divergenee, the wall-normal advection-diffusion equation is solved subject to a homogenous Neumann condition, while the boundary conditions on the wall-parallel velocity components remain unchanged. The modified scheme for the wall-nomal equations comprises the following steps:

1. Solve the advection-diffusion equation subject to the homogeneons Neumann boumelary condition:

$$
\frac{\partial u_{r}^{*}}{\partial r}=0
$$

2. Recover the intermediate wall-normal velocity at the no-slip boundary.

3. To ensure no-penetration at the full time step, solve the pressure Poisson equation subject to the Neumann boundary condition:

$$
\widehat{n} \cdot \nabla \Phi^{n+1}=\frac{u_{r}^{\star}}{\Delta t}
$$


Equation (3.4) like the commonly used homogencous condition on pressure - has no physical basis, but is used as a convenient means of ensuring that the no-penctration condition is satisficd.

The effect of employing a homogeneous Dirichlet versus Neumann boundary condition is illustrated for the uniform flow past a stationary sphere at $R e=100$. For each fractional-step method considered, table 3.1 presents the total drag coellicient, $C_{D}$, separation angle, $\theta_{S}$, wake length, $L_{W}$, maximum surface divergenee, $\left.\nabla \cdot u\right|_{\text {max }}$, and root-mean-square surface divergenec, $\left.\boldsymbol{\nabla} \cdot \boldsymbol{u}\right|_{\text {rns }}$. For all three cases, using the Neumann condition rather than the Dirichlet condition modifies $C_{D}$ by less than 0.15 percent, and the effect on $\theta_{S}$ and $L_{W}$ is smaller still. For Fsm I and Fsm II, a Noumann boundary condition reduces both $\left.\boldsymbol{\nabla} \cdot \boldsymbol{u}\right|_{r m s}$ and $\left.\boldsymbol{\nabla} \cdot \boldsymbol{u}\right|_{\text {rrax }}$ by over onc order of magnitude. The effect is more pronounced for Fsm III, where a reduction of five orders of magnitude is found. The marked improvement for this method is due to the presenee of a lagging pressure gradient term in the advection-diffusion equations, which results in the intermediate and projected velocity fields differing by, at most, $\mathcal{O}\left(\Delta l^{2}\right)$.

Figure 3.4 presents the pressure coefficiont along a radial cut near the equator of the sphere. For F'sm I, a Dirichlet boundary condition results in a 'kick-up' adjaccent, to the boundary. This numerical boundary layer is a well-known artifact of the mothod. For l'sm II and Fsm III, the inclusion of the Kim \& Moin (1985) crror-absorbing werm greatly reduces the extent of the numerical boundary layer. When the Neumann bonndary condition is used, all three fractional-step melhods show close agreenont, with litule evidence of a numerical boundary layer. Figure 3.5 presents the wall-normal velocity along a radial cut near the equator of the sphere. The effect of the different projection methods and boundary conditions is small and confined to a few grid points adjacent to the solid surface.

Overall, a Neumann condition for the wall-normal velocity favorably affects the surface divergenee. However, it was also shown that the choice of bounclary condition has only a very localized effect and produces insignificant changes in the cuantitics 
of interest such as the drag coefficient, separation angle and wake length. For this reason, in the remainder of this study, Fsm II is chosen in conjunction with a Dirichlet boundary condition for $u_{r}^{*}$. This generally accepted discretization is cmployed becausc of its computational efficiency and to avoid any doubts that may arise in the reader from the use of the new and relatively untested scheme proposed here.

\begin{tabular}{lcccccc}
\hline Fsm & b.c. & $C_{D}$ & $\theta_{S}$ & $L_{W}$ & $\left.\nabla \cdot u\right|_{\max }$ & $\left.\nabla \cdot u\right|_{r m s}$ \\
\hline I & Dirichlet & 1.0873 & 53.214 & 1.7371 & $3.0695 \times 10^{-2}$ & $5.0497 \times 10^{-4}$ \\
I & Neumann & 1.0886 & 53.214 & 1.7371 & $5.5283 \times 10^{-4}$ & $8.2879 \times 10^{-6}$ \\
II & Dirichlet & 1.0881 & 53.214 & 1.7371 & $3.0695 \times 10^{-2}$ & $5.0497 \times 10^{-4}$ \\
II & Neumann & 1.0886 & 53.214 & 1.7371 & $5.5283 \times 10^{-4}$ & $8.2879 \times 10^{-6}$ \\
III & Dirichlet & 1.0881 & 53.214 & 1.7371 & $3.0670 \times 10^{-2}$ & $5.0474 \times 10^{-4}$ \\
III & Neumann & 1.0886 & 53.214 & 1.7371 & $1.8892 \times 10^{-7}$ & $1.8956(i) \times 10^{-9}$ \\
\hline
\end{tabular}

Table 3.1: The effect of applying a homogencous Dirichlet versus a homogeneous Nenmann boundary condition (b.c.) for the intermediate wall-normal velocity.

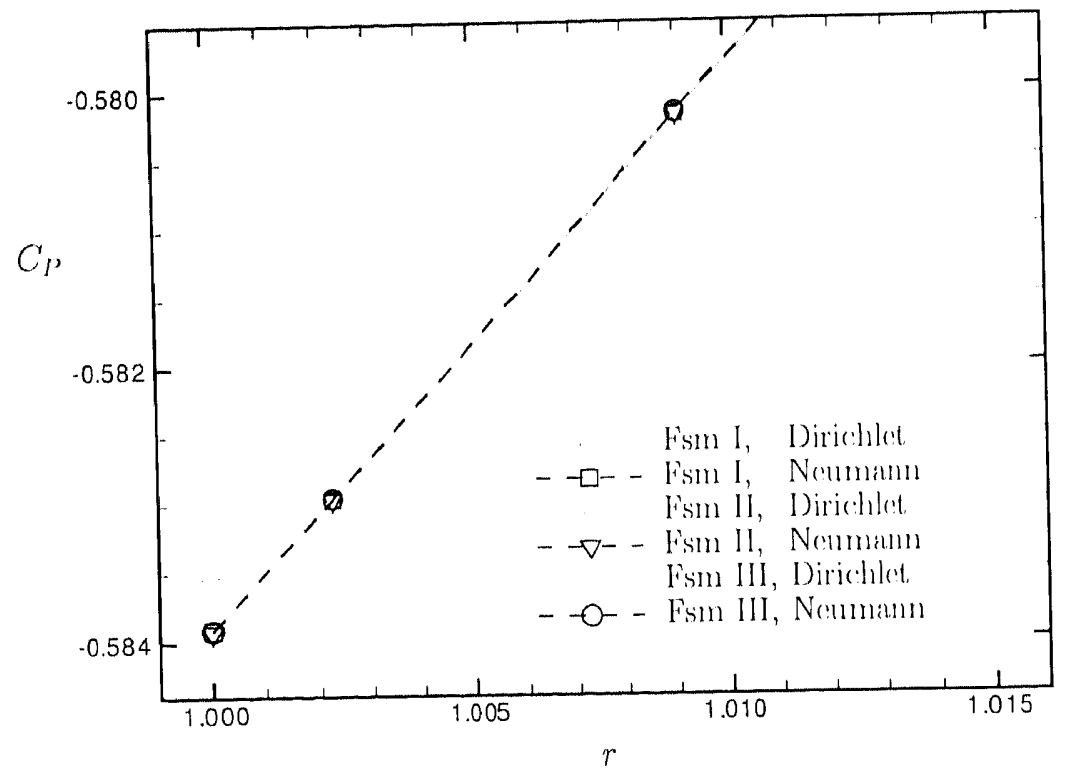

Figure 3.4: Pressure coefficient, $C_{P}$, along a ray near the equator of the sphlere cquator $(\theta=0.501 \pi)$ for $R e=100$ and $\Delta t^{*}=0.0002$. 


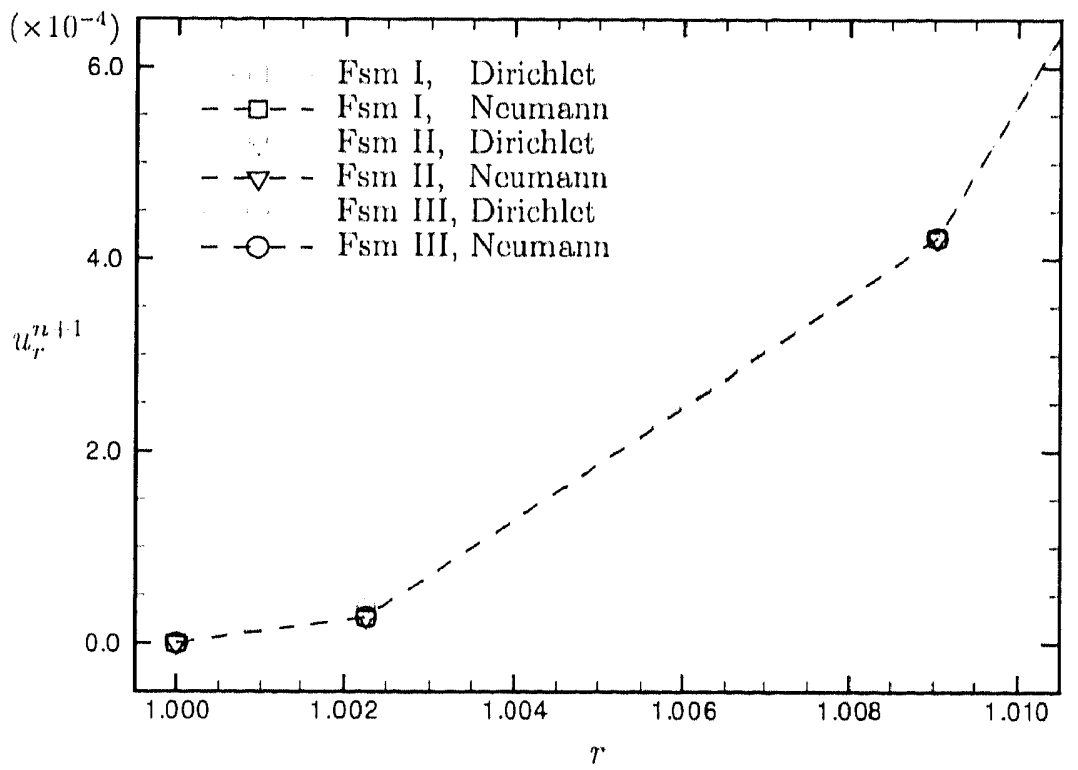

Figure 3.5: Raclial velocity along a ray near the equator of the sphere $(\theta=0.501 \pi)$ for $R\left(:=100\right.$ and $\Delta l^{*}=0.0002$. 


\subsection{Sensitivity to spatial and temporal resolution}

Insensitivity to spatial and temporal resolution was verificd for all Reynolds numbers and rotation rates considered. Numerous preliminary simulations were undertaken to establish the most suitable grid parametcrs. The grid clustering in the near-wall region was tailored to cnsure that at least twelve to fifteen collocation points resicle in the boundary laycr, while clustering in the $\theta$-direction was tailored to most efficiontly resolve the high gradients in the wake. The adequacy of spatial and temporal resolution was verified by increasing the grid resolution and reducing the time-step and confirming that important parameters, such as the force coefficients, scparation angle and shedling frequency, changed by less than 1 percent. Moreover, the primitive variables were transformed into wavenumber space and it was verificd that the expansion coefficionts decayed by at least 4 to 5 orders of magnitude. The spatial and temporal parameters relevant to the simulation results presented in chapters 5 and 6 are shown at the outset of these chapters. In this section, some of the measures taken to ensure that, the simulations were adequately resolved are illustrated using the stalionary sphere at, $R e=100$ and $R e=300$ as cxamples.

For $R e=100$, the effect of $\Delta t^{*}$ on the drag cocflicient, separation angle and wake: length are presented in table 3.2. In this and subsecunent, hables, the crore is calculated relative to the highest resolution case tabulated, and the parameter value juclged to be adecpuate is highlighted in grey. The effect of $N_{r}$ and $N_{0}$ is presented in table 3.3 and table 3.4, respectively. The offect of clomain size is presented in lable 3.5. Ne this lower Reymolds number, diffusion effects are expected to be more significant and the demain should be of sufficient size so as to minimize blockage effect.s. With increasing D) o/d, the radial stretching parameter, $x_{\text {min }}$, is also varied to maintain a similar distribution of collocation points near the sphere surface.

For $R e=300$, the cffect of domain size on the time-averaged drag and lift and on the vortex shedding Strouhal number is presented in table 3.6. It is well-known that 
the vortex shedding Strouhal number is particularly sensitive to computational domain size. For the chosen value of $D_{\mathrm{o}} / d=45$, the parameters listed show a relative error of less than 1 percent. It is also worth noting that for the same test condition, Johnson \& Patel (1999) and Kim \& Choi (2002) used $D_{\mathrm{o}} / d=30$. In the present study, a larger domain was chosen to allow a greater streamwise wake development, before the structures exit the domain.

The effect of $\Delta t^{*}$ for $R e=300$ is presented in table 3.7. For this unsteady flow, the chosen $\Delta t^{*}=5.0 \times 10^{-4}$ is several orders of magnitude smaller than the smallest. time scale in the primitive variables. The effect of $N_{\phi}$ is prescnted in table 3.8 and the instantancous wake structure, as identified using the vortex identification method of Jeong \& Hussain (1995), is compared in figure 3.6. The effect on the force codficients and Strouhal number is negligible. This is reflected in the wake strueture for $N_{\phi}=32$ and 64 being practically indistinguishable. Finally, ligure 3.7 presents typical spectral modes versus wavenumber along the threc coordinate directions. For cach combination of $r, \theta$ and $\phi$, the grid resolution was juclged to be adequate whon al least 4 to 5 decarles decay in the expansion coefficients was found.

\begin{tabular}{ccccccc}
\hline$\Delta t^{*}$ & $C_{L}$ & $\mid$ Error $(\%) \mid$ & $O_{S}(\mathrm{deg})$ & $\mid$ Error $(\%) \mid$ & $L_{W}$ & $\mid$ Error $(\%) \mid$ \\
\hline $5 \times 10^{-4}$ & 1.0881 & $1.9028 \times 10^{-2}$ & 53.214 & $3.4577 \times 10^{-4}$ & 0.8691 & $1.1506 \times 10^{-3}$ \\
$1 \times 10^{4}$ & 1.0879 & $2.8037 \times 10^{-3}$ & 53.214 & $1.2591 \times 10^{-4}$ & 0.8691 & $4.6023 \times 10^{-4}$ \\
$5 \times 10^{-5}$ & 1.0879 & - & 53.214 & - & 0.8691 & - \\
\hline
\end{tabular}

Table 3.2: Sonsitivity to $\Delta l^{*}$ for $R e=100$.

\begin{tabular}{ccccccc}
\hline$N_{r}$ & $C_{D}$ & $\mid$ Error (\%)| & $O_{S}$ (deg.) & $\mid$ Error (\%)| & $L_{W}$ & $\mid$ Error (\%) $\mid$ \\
\hline 81 & 1.0868 & $8.8558 \times 10^{-2}$ & 53.216 & $4.1662 \times 10^{-3}$ & 0.8692 & $1.0355 \times 10^{-2}$ \\
121 & 1.0881 & $1.2415 \times 10^{-2}$ & 53.214 & $4.5477 \times 10^{-4}$ & 0.8691 & $9.2048 \times 10^{-4}$ \\
161 & 1.0885 & - & 53.214 & - & 0.8691 & - \\
\hline
\end{tabular}

Table 3.3: Sensitivity to $N_{r}$ for $R e=100$. 


\begin{tabular}{ccccccc}
\hline$N_{0}$ & $C_{D}$ & $\mid$ Error (\%)| & $\theta_{S}$ (deg.) & $\mid$ Error (\%)| & $L_{W}$ & $\mid$ Error (\%)| \\
\hline 80 & 1.0879 & $3.6777 \times 10^{-4}$ & 53.200 & $3.5645 \times 10^{-2}$ & 0.8683 & $7.5193 \times 10^{-4}$ \\
100 & 1.0881 & $3.1904 \times 10^{-2}$ & 53.214 & $1.0043 \times 10^{-2}$ & 0.8686 & $3.9092 \times 10^{-4}$ \\
160 & 1.0879 & - & 53.219 & - & 0.8689 & - \\
\hline
\end{tabular}

Table 3.4: Scnsitivity to $N_{\theta}$ for $R e=100$.

\begin{tabular}{cccccccc}
\hline$D_{0} / d$ & $x_{\min }$ & $C_{D}$ & $\mid$ Error (\%)| & $\theta_{S}$ (deg.) & $\mid$ Error $(\%) \mid$ & $L_{W}$ & $\mid$ Error $(\%) \mid$ \\
\hline 15 & 0.24 & 1.0927 & $4.6090 \times 10^{-1}$ & 53.235 & $4.2261 \times 10^{-2}$ & $0.8695)$ & $4.9478 \times 10^{-2}$ \\
30 & 0.20 & 1.0885 & $7.8701 \times 10^{-2}$ & 53.218 & $1.0417 \times 10^{-2}$ & 0.86903 & $2.34473 \times 10^{-2}$ \\
45 & 0.30 & 1.0879 & $1.8112 \times 10^{-2}$ & 53.214 & $2.8133 \times 10^{-3}$ & 0.8691 & $5.8683 \times 10^{-3}$ \\
60 & 0.30 & 1.0877 & - & 53.213 & - & 0.8691 & - \\
\hline
\end{tabular}

Table 3.5: Scnsitivity to $D_{0}$ for $R e=100$.

\begin{tabular}{cccccccc}
\hline$D_{\mathrm{o}} / d$ & $x_{\min }$ & $\overline{C_{D}}$ & $\mid$ Error $(\%) \mid$ & $\overline{C_{L}}$ & $\mid$ Error $(\%) \mid$ & $S t$ & $\mid$ Error $(\%) \mid$ \\
\hline 30 & 0.20 & 0.6587 & $3.8737 \times 10^{-2}$ & 0.06724 & 1.0035 & $0.134(1)$ & $9.30)\left(88 \times 10^{-2}\right.$ \\
45 & 0.30 & 0.6584 & $1.4565 \times 10^{-2}$ & 0.06701 & 0.6579 & 0.13389 & $1.8596 \times 10^{-2}$ \\
60 & 0.40 & 0.6585 & - & 0.06657 & - & 0.133392 & - \\
\hline
\end{tabular}

Table 3.6: Sensilivity to $D_{0}$ lor $R e=3(0)$.

\begin{tabular}{|c|c|c|c|c|c|c|}
\hline$\Delta l^{*}$ & $\overline{C_{D}}$ & $\mid$ Frror $(\%) \mid$ & $\overline{C_{1}}$ & $\mid$ Error $(\%) \mid$ & $S ' l$ & $\mid$ Wrror $(\%) \mid$ \\
\hline & () & $2 \times 10^{2}$ & $0.067(1)$ & $\times 10^{2}$ & 39 & $8.5(1) 70 \times 10^{3}$ \\
\hline $5.0 \times 10^{-4}$ & 0.6584 & $1.2607 \times 10^{-2}$ & 0.0670 & $8.3910 \times 10^{-3}$ & 0.13399 & $1.0202 \times 10^{-2}$ \\
\hline $2.5 \times 10^{4}$ & 0.6583 & - & 0.0670 & - & 0.13339 & - \\
\hline
\end{tabular}

Table 3.7: Sensilivily to $\Delta l^{*}$ for $R\left(c^{2}=300\right.$.

\begin{tabular}{ccccccc}
\hline$N_{\phi}$ & $\overline{C_{D}}$ & $\mid$ Error $(\%) \mid$ & $\overline{C_{L}}$ & $\mid$ Error $(\%) \mid$ & $S !$ & $\mid$ Hrror $(\%) \mid$ \\
\hline 32 & 0.6584 & $3.4937 \times 10^{-4}$ & 0.0670 & $2.9997 \times 10^{-03}$ & 0.1339 & $2.1655 \times 10^{-2}$ \\
64 & 0.6584 & - & 0.0670 & - & 0.1339 & - \\
\hline
\end{tabular}

Table 3.8: Sensitivity to $N_{\phi}$ for $R e=300$. 


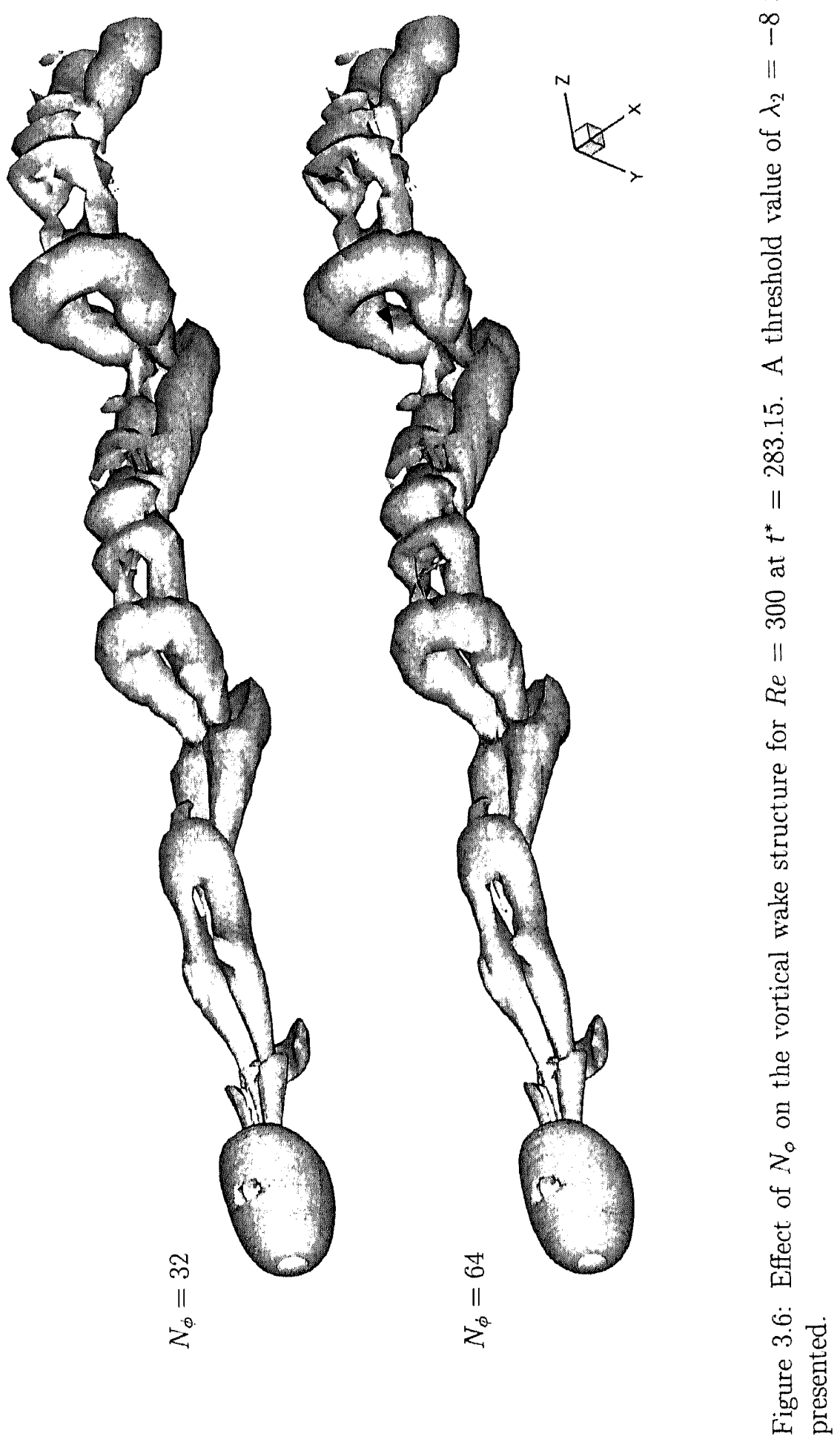



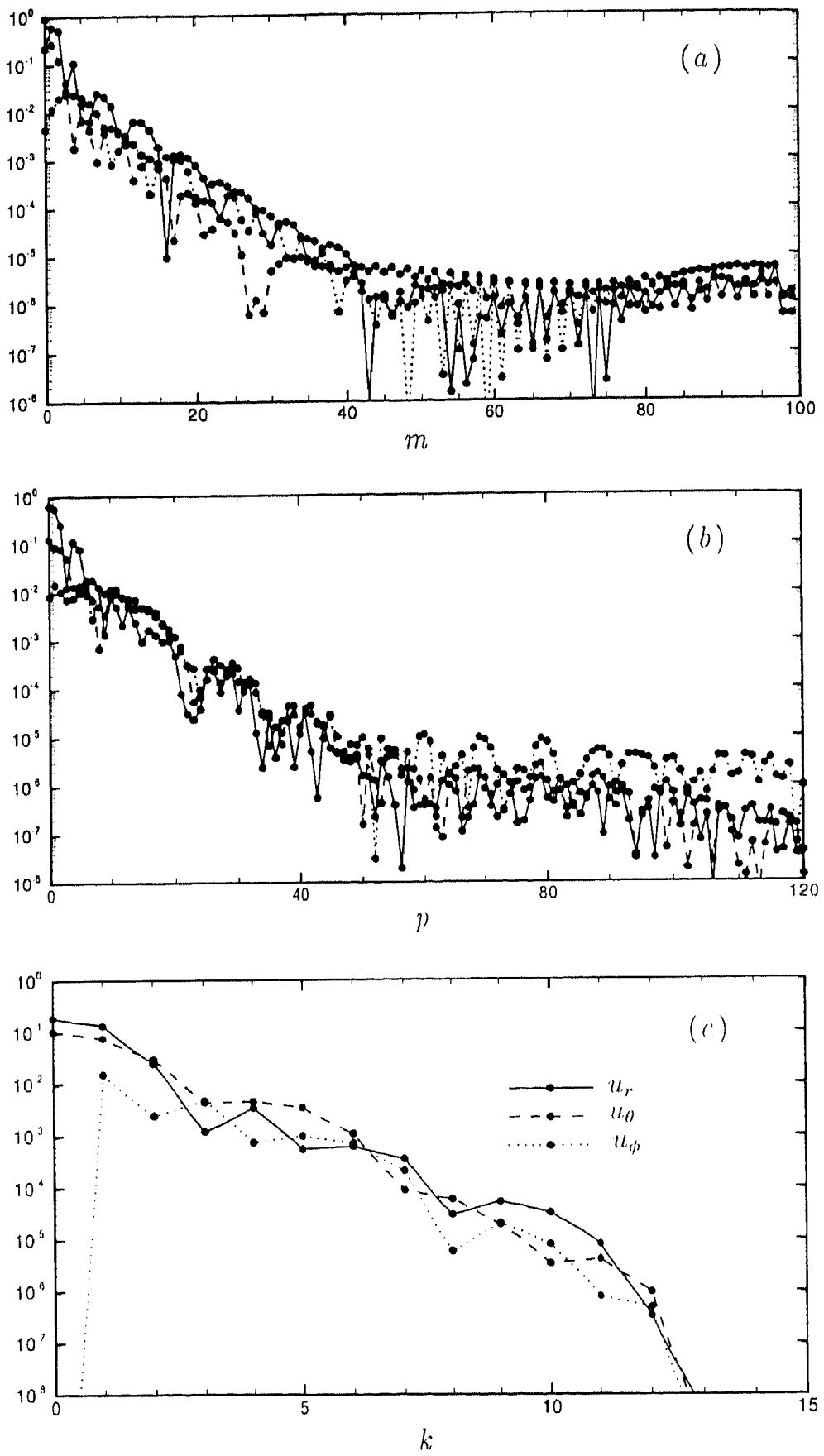

Figure 3.7: Instantancous spectral modes of the spherical velocity components for $R e=300:(a)$ radial spectrum along $(\theta, \phi)=(0.056 \pi, 0.188 \pi) ;(b)$ tangential spectrum along $(r, \phi)=(1.92,0.188 \pi) ;(c)$ azimuthal spcctrum along $(\theta, \phi)=(0.056 \pi, 0.188 \pi)$. 


\subsection{Performance of the outflow boundary condition}

As the outflow boundary is approached, the radial diffusion terms of the Navier Stokes equations are smoothly attenuated to zero by multiplying them with the filter function, $g(r, \theta)$, given by equation (2.61). This renders the governing equations parabolic near the outflow boundary to ensure that disturbances are convected out of the domain without reflection.

The filter is adapted from that developed by Mittal (1995) for the study of the flow past two- and three-dimensional cylinders. To test the performance of the outflow filter, Mittal devised a numerical experiment that allows an isolated vortical structure to be studied as it convects downstream. The filter function could then be optimized to ensure minimal distortion of the vortical structures as they eutered the filtered region. This experinent was repeated for the grid topology and filter function combination used in this study. The simulation starts with the axisymmetrice, steady-state flow for a sphere at $R e=200$ (which corresponds to $l^{*}=250$ ). At this instant the no-slip conclition at the sphere surface is impulsively replaced by the potential flow velecities, causing the wake to detach from the surface and convect downstream. A comparison of the evolution of the vortical structure with outflow filtering and the referenec case of no filtering is presented in figure 3.8, in which a time sequence of the $x$-vorticity on the $(y, z)$-plane is plotted. The unfiltered case is shown in the left panel and the filtered (atse is shown in the right panel. $A$ linear grey seale color map is used to inclieate the degree of fillering, with white signifying no filtering $(g(r, \theta)=1.0)$ and black signifying Cull liltoring $(g(r, \theta)=0.0)$.

As the detached structure convects downstream, it decays due to viscons dissipation. $\Lambda$ comparison of the filtered and unfiltered time sequences reveals that filtering canses little cleformation of the structures as they approach the outflow boundary. Figure 3.9 compares the $x$-vorticity $\left(\omega_{x}\right)$ along a radial cut through $\theta=0.011 \pi, \dagger$

$\dagger$ This coordinate was chosen so that the radial cut passed through the region of highest, vorticity in the vortical structure. 
The unfiltered and filtered cases are indistinguishable upstream of the filtered region and show only minor differences in the vorticity profile when the structure enters the filtered region. Importantly, no spurious vorticity is apparent as the structure exits the domain. Although not apparent in figures 3.8 and 3.9, the solution comprising no outflow filtering is contaminated by reflected waves of very small amplitude. Such reflected disturbances can become more significant when more general unsteady flows are simulated.

The effect of the outflow filter on the vortical wake may be further scrutinized simply by considering the effect of an increased domain size, while maintaining the same outflow filter function and spatial resolution (this creates a greater unfiltered region). Such tests were considered for a number of flow cases and the effect on the wake evolution was always indiscernible. In $\S 3.2$, the effect of domain size was illustrated for a stationary sphere at $R e=100$ and $R e=300$. An increase in the outer boundary size from $D_{0} / d=30$ to $D_{\circ} / d=60$ showed very little effect in a number of important, flow quantitics. 


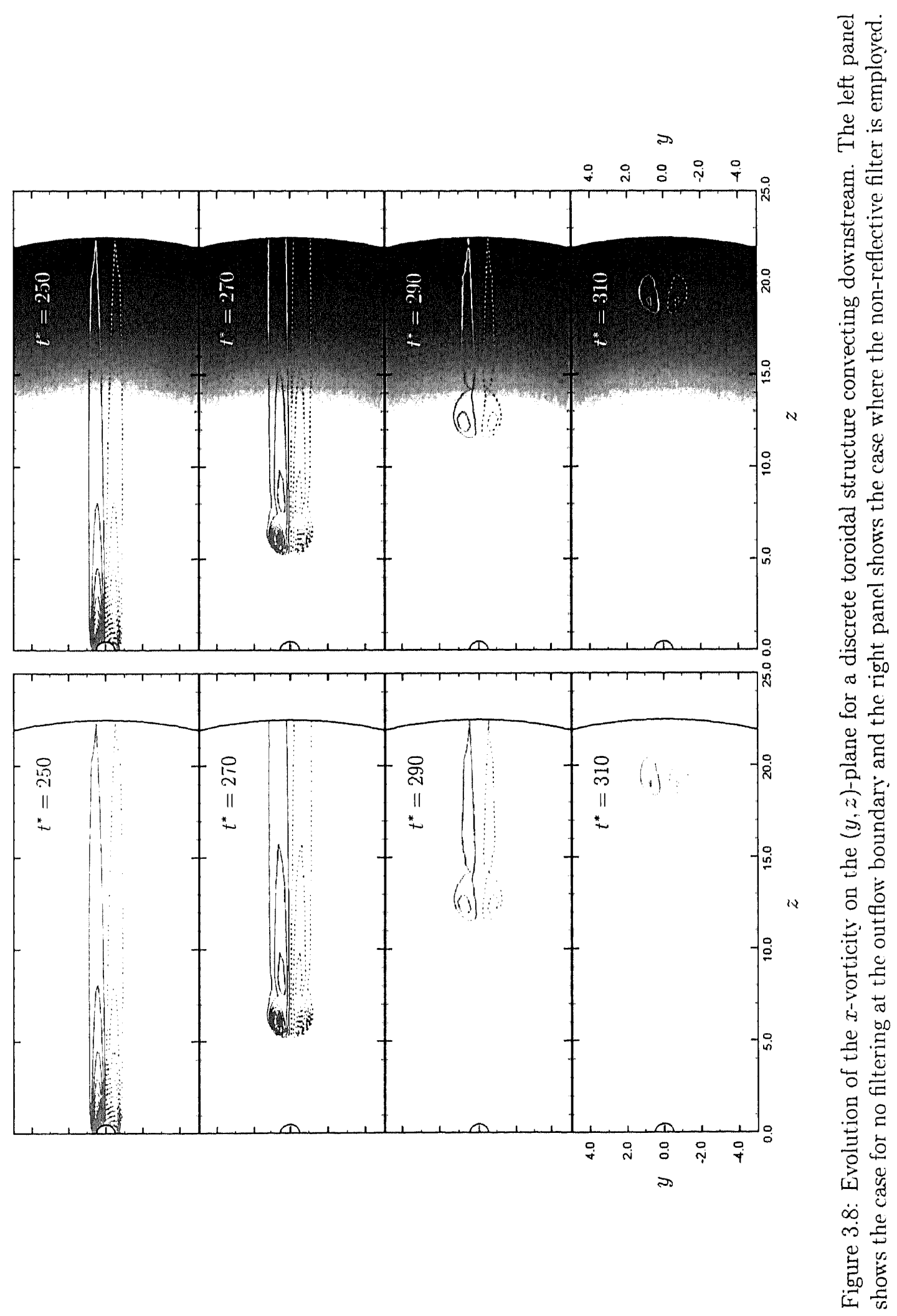




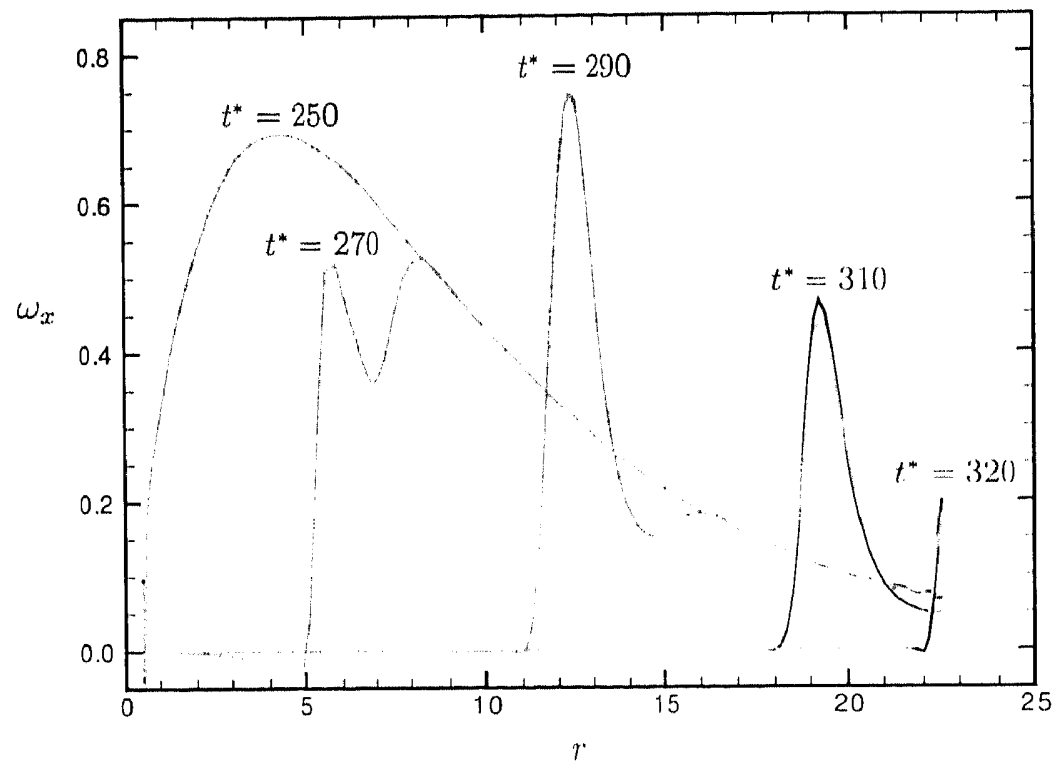

Figure 3.9: Evolution of the $x$-vorticity along a raclial cut through $0=0.011 \pi$ for a discrete toroidal structure convecting downstream: , ontllow liltoring; , 110 ontflow filtering. In the near-fick the profiles are inclistinguishable. 


\subsection{Comparison with earlier studies}

For the uniform flow past a stationary sphere, a number of studics are available for comparison. Reliable data is scarce for a spinning sphere and a rigorous validation is possible only against the numerical study of Kim \& Choi (2002) for a sphore in streamwise rotation. Results for both a stationary and rotating sphere are organized in increasing Reynolds number order and cover the axisymmetric steady, asymmotric: stcady and vortex shedding regimes.

\subsubsection{Axisymmetric regime, $20<R e<212$}

In the range $R e=20$ to 212 , the uniform flow past a stationary sphere remains axisymmetric and steady and is characterized by a closed toroidal wake structure. Figure 3.10 presents the separation angle, $\theta_{S}$, and wake length, $L_{W}$, with respect to the Reynolds number. Both the separation angle and wake length are measured relative to the rear stagnation point and the wake length is normalized by the sphere diameter. The results are in close agrecment with those reported by Tomboulides \& Orszag (200()). The separation angles measured experimentally by Nakamura (1976) are also in elose agreement, at high Reynolds number, but show higher values at the lower Reynolds numbers. The wake length measuremonts of Tancela (1956) are in agreement, up to a Reynolds number of about 130. At Reynolds numbers above 130, Timeda (1956) reported faint periodic oscillations in the recirculating wake and, ats noted by Tomboulides \& Orszag, this may be the cause of the discrepancy at higher Reynolds numbers.

The total drag coefficient, $C_{D}$, is presented as a function of the Reynolds number in ligure 3.11 and shows good agreement with the experimentally measured results of Roos \& Wilhmarth (1971) and excellent agreement with the computed values of Johnson \& Patel (1999). In general, integrated quantities such as the drag coefficiont are less sensitive than local quantitics, and for this reason figure 3.12 compares the pressire coefficiont at the downstream pole of the sphere, $C_{P b}$, versus Reynolds number, with 
that presented by Tomboulides \& Orszag (2000). Here, the pressurc coefficient is defined as

$$
C_{p}=\frac{P-P_{\infty}}{\frac{1}{2} \rho U_{\infty}^{2}}
$$

where the ' $\infty$ ' subscript significs conditions in the incoming frecstrcam flow. Although all other diagnostics show excellent agreement with the result, of Tomboulides \& Orszag, the deviation in base pressure is as much as 11 percent. This difference may be attributed to the different far-ficld boundary conditions imposed in the two studies. In the study of Tomboulides \& Orszag, the flow was calculated in a cylinclrical domain which extends 4.5 diameters outwards from the center of the sphere and at, this boundary the velocity is prescribed as the freestream velocity. In the present studly, the onter domain extends to 22.5 diameters from the sphere conter and the bonndary conditions are constructed with the aim of minimizing the blockage effects of the outer boundary. Modi \& Akutsu (1984) showed that increasing blockage reduces the base pressure and provides a possible explanation for the discrepancy secen in figure $3.12 . \dagger$

For a sphere rotating in the streamwise direction at $R r=100$, Kim \&: Choi (2002) found the wake to remain axisymmetrie over the range $\Omega^{*}-(0.0)$ l. 1.0 . The drag coefficient versus non-dimensional spin rate for these test conclitions are compared in figure 3.13. The increase in drag coefficicul with increasing $\Omega^{+}$reflects the increase in the size of the low pressure wake. The agrecment with the results of Kim do (Got is cxcollent. Figure 3.14 presents the separation angle and wake lenghth versuse $2^{*}$. The agreonent in the predicted wake length is excellent. While the separation angles show similar tronds, they differ by as much as lwo degreses. Alse shown are the values for a stationary sphere computed by Johnson \& Patel (1999) and measured experimentally by Nakamura (1976). These are in close agrecment, with the values derived in the present, study and provide a strong validation of the results of this study. $\Lambda$ notable dif-

$\dagger$ In the present study, a domain sizo sensitivity study found that relucing the domein sizo reduced the base pressure coefficient. For example, at $R e=100$, domain sizes of $D_{0} / d=15,30,45$ and 60 result in $C_{P b}=-0.155,-0.149,-0.148$ and -0.148 , respectively. 

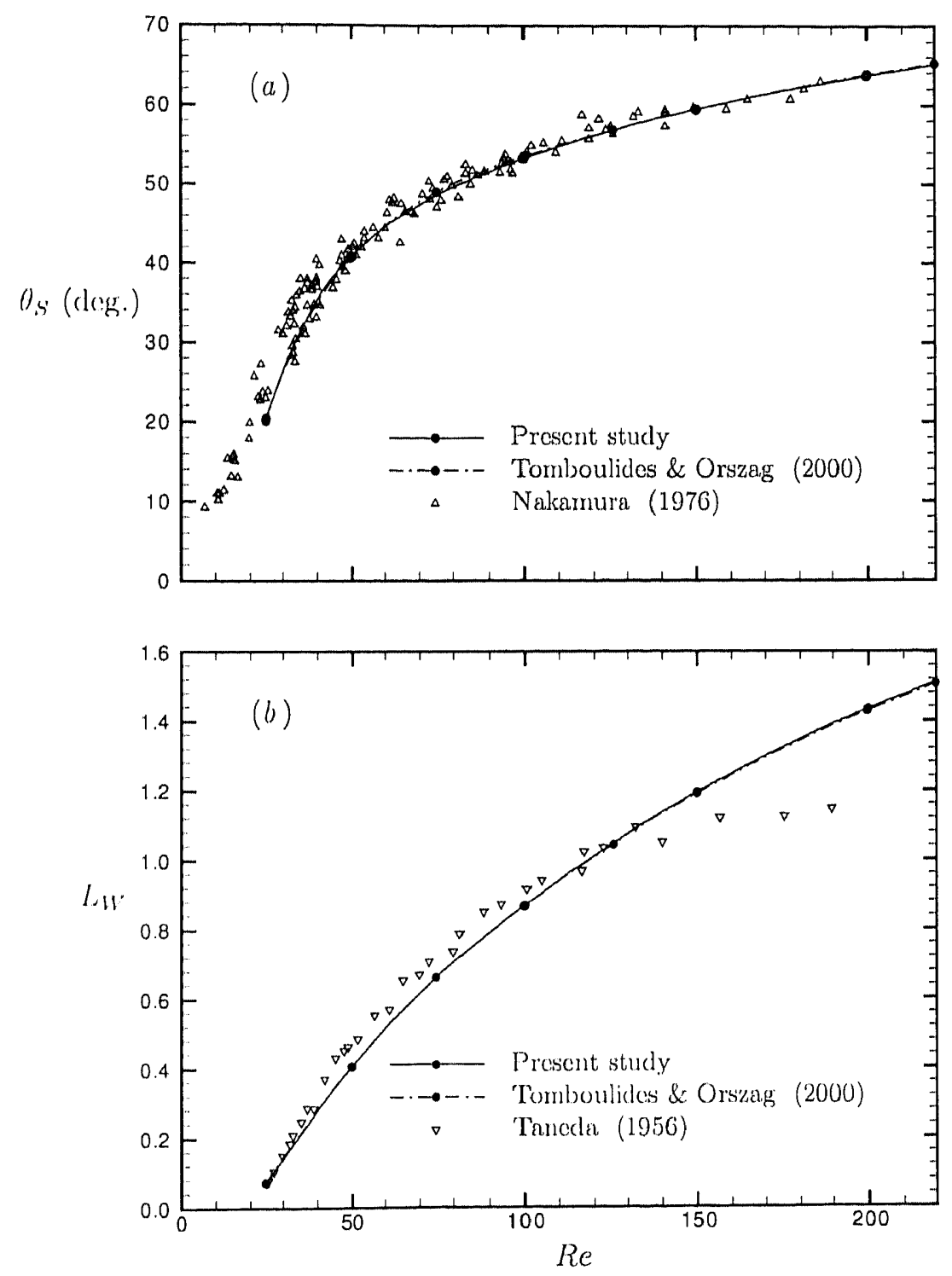

Figure: 3.1(): (a) Separation angle, $\theta_{S}$, and (b) Wake length, $L_{W}$, with respect to Re. 
ference between the present study and that of Kim \& Choi is their usc of an immersed boundary method to model the solid boundary. $\dagger$ The immersed boundary method allows a computational grid that is not conformal to the surface of the body and the no-slip and no-penetration boundary conditions are enforced by applying a suitable momentum forcing in the Navier-Stokes equations. This has a number of advantages over more conventional methods of solution, particularly for complex geometries and problems involving moving boundaries. However, as discussed by Mit,al \& laccarino (2005), body-conformal grids allow better control of the grid resolution in the vicinity of the body. They also highlight a number of numerical issues in the boundary treatment which may degrade the accuracy with which the boundary conditions are enforeed for immersed boundary methods. In light of this, it appears that the discrepancy in the separation angle predictions may be linked to the immorsed bounclary treatiment.

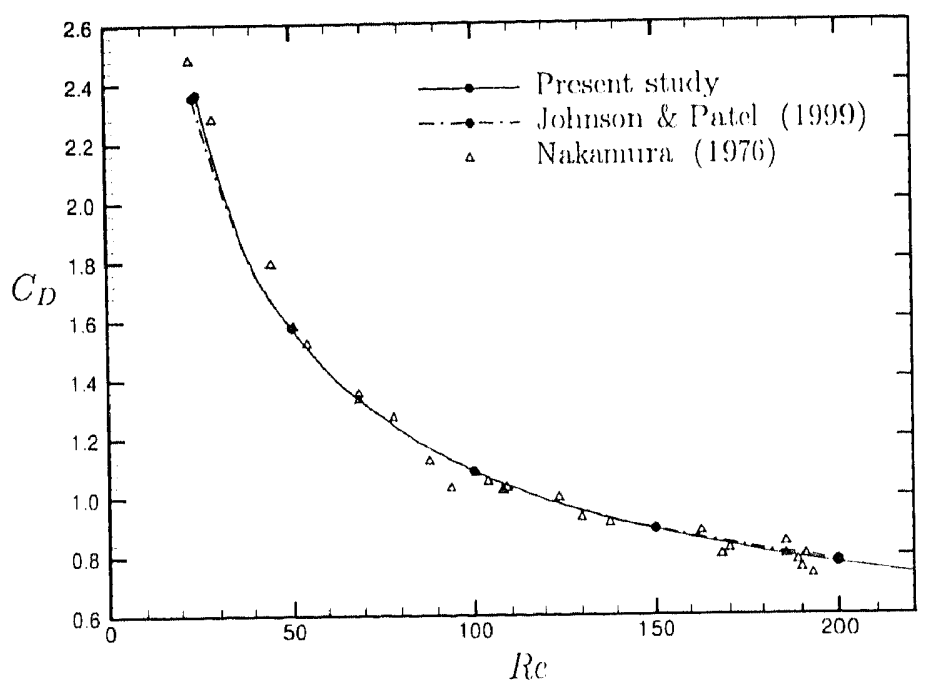

Figure 3.11: Drag coefficient, $C_{D}$, with respect to Re:

$\dagger$ The sensitivity studies presented earlier in this chapter suggest that other important parameters, such as the temporal resolution and domain size, are adequate and should not affect the separation angle. 


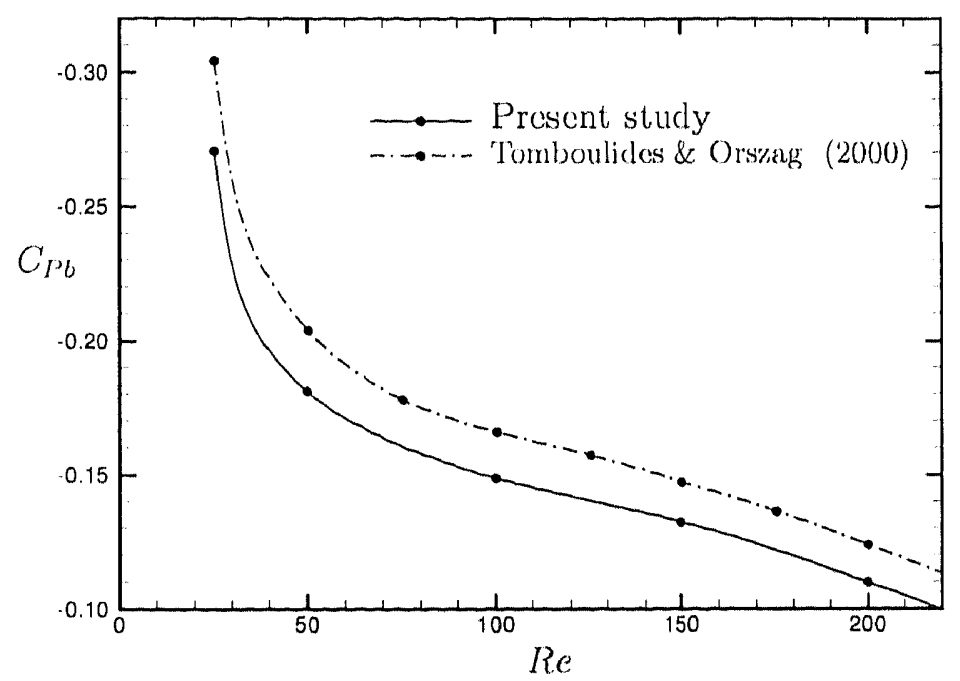

Figure 3.12: Base pressure coefficiont, $C_{P^{\prime} b}$, with respect to Re.

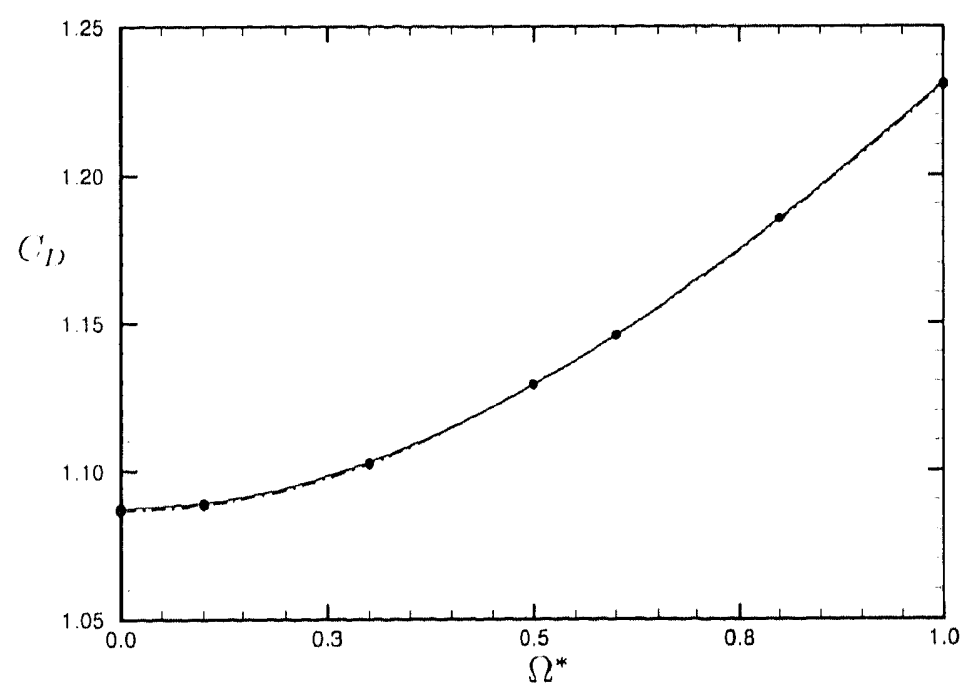

Figure 3.13: Drag coefficient, $C_{D}$, for $R e=100$ with respect to $\Omega^{*}$ : - - Present, study; - - K Kim \& Choi (2002). 

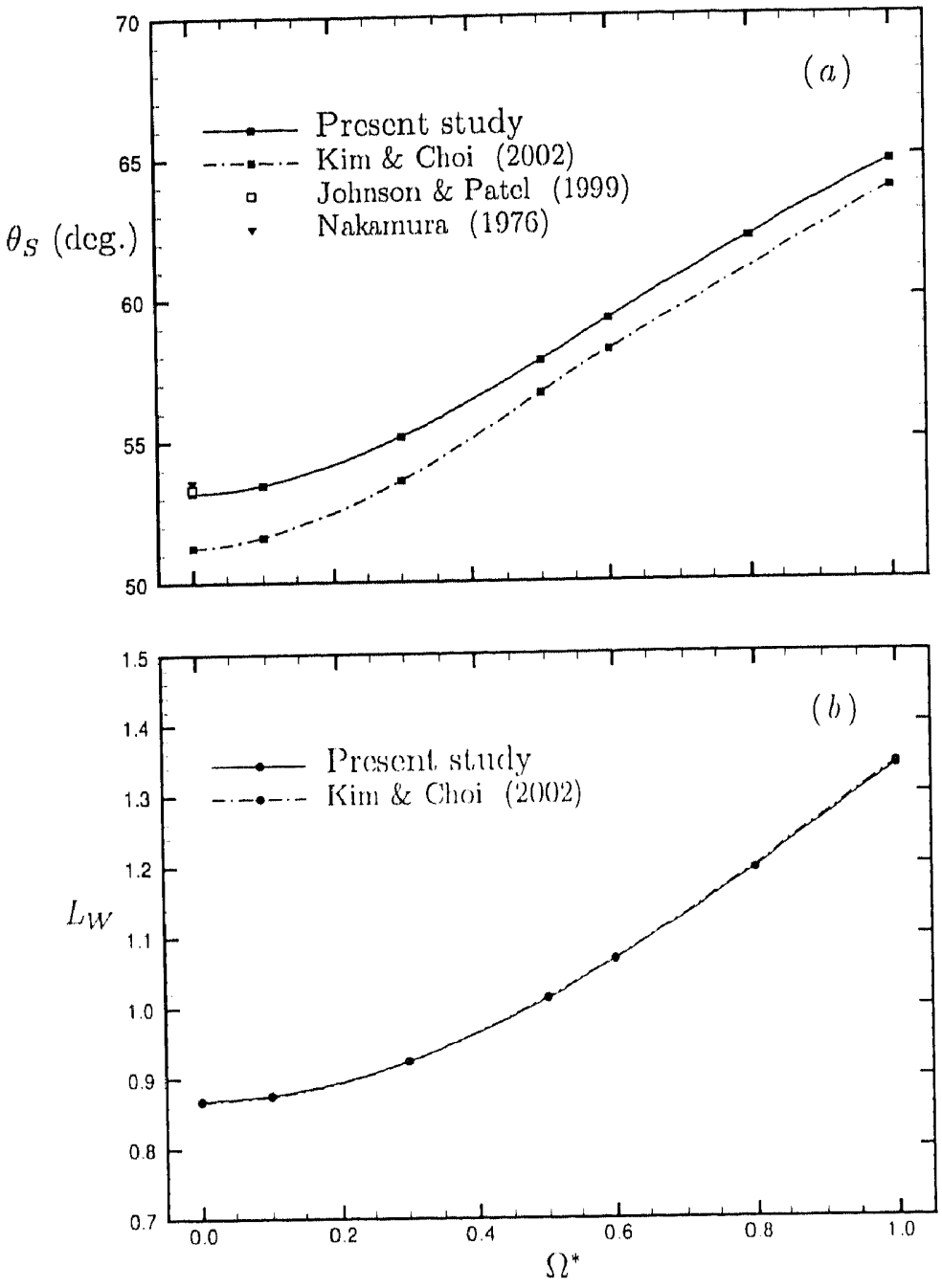

Figure 3.14: (a) Separation angle, $\theta_{S}$, and $(b)$ Wake length, $L_{W}$, and with respect, to $\Omega^{*}$ for $R c=100$. 


\subsubsection{Asymmetric steady regime, $212<R e<270$}

Above $R e=212$, the wake behind a stationary sphore undergoes a regular bifurcation to an asymmetrie, steady state. In this regime the flow remains topologically similar with increasing Re, until a Hopf bifurcation at $R e \approx 270$. Figure 3.15 presents contours of the $x$-component of vorticity, $\omega_{x}$, on the flow symmetry plane for Re $=250$. The loss of axisymmetry is highlighted by the contours of positive vorticity extending further downstream than those of negative vorticity. In tho study of Johnson \& Patel (1999), the flow symmetry plane was rotated to coincide with the $(x, y)$-plane, while here the flow is symmetric about the $(y, z)$-plane. Henee it is appropriate to compare against, their $z$-component of vorticity, $\omega_{z}$, on the $(x, y)$-plane. Excellent, agreement is secn in the near-wake, but this degrades further downstream. In both studics a spherical grid lepologgy is used, which produces a lower griel resolution further away from the origin of the coordinate system. Although the grid resolution in the wake is adequate to resolve the flowfield using spectral expansions, using lower-order plotting algorithms with the sanne distribution of grid points leads to poor ficlelity. This may explain the differences evident, here. The foree coefficients are compared with those of other muncrical studies in table 3.9 of the following section. The separation angle and wake length are not, reported for the asymmetric and vortex shedding regimes because the wake is no longer closed and the separation angle is a function of the azimuthal angle, $\phi$. In figure 3.16, the wake strueture for $R e=250$ is compared with that presented by Kim \& ('hei (20)2). The wake strueture, us identified using the mothod of Jeong \& Hussain (1995), is shown for both a stationary sphere and a sphere in streamwise rotation with $S^{*}=0.10$. For the present study iso-surfaces of $\lambda_{2}=-8 \times 10^{-4}$ are shown, while the threshold value used by Kim \& Choi is not specified. $†$ For a stationary sphere, the flow strueture is characterized by a mushroom shroud that envelopes the sphere and at wake comprising two tails that are equal in streamwise vorticity magnitude, but opposite in sign the so-called 'double-threaded' wake. Sphere rotation introduces a positive

$\dagger$ In chapter 4 , it will be shown that the $\lambda_{2}$ definition is insensitive to the threshold value used. 

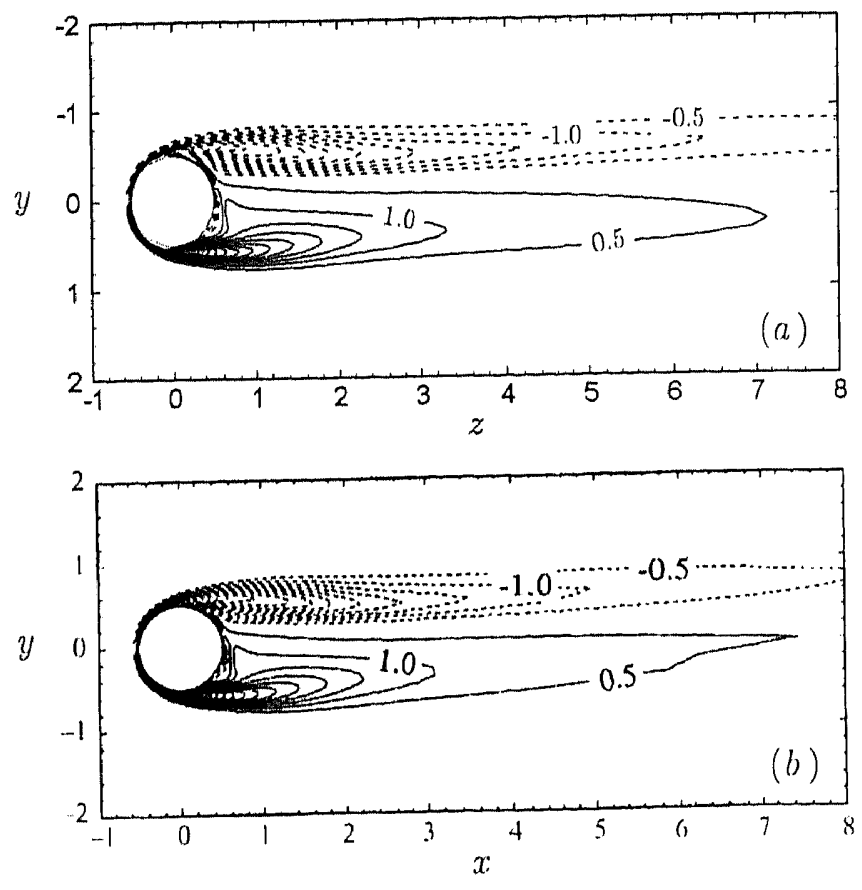

Figure 3.15: Vorticity contours for $R e=250$. (a) $\omega_{\text {ir }}$ in the $(y, z)$-plane from the present study. $(b) \omega_{z}$ in the $(x, y)$-plane taken from Johnson \& Patel (1999). Contours of negative vorticity are indieted by dashed lines.

streamwise vorticity in the boundary layer, which is convected into the wake and works to strengthen the tail of pesitive vorticity and weaken the negative vorticity tail. The agrement between the studies is excellent. Figure 3.17 compares the force coefficiont

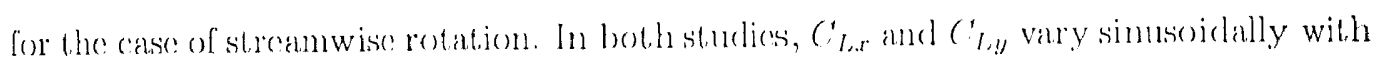
the same amplitude and frecuency, but show a small phate difference. This discrepancy may be altributed to differences in the initial conditions. Kim \& (Choi initialized the domain with the flowfield computed for a stationary splere, while in the present study the flow was initialized with a potential flowficld. $C_{D}$ and the net lateral forec, $C_{L}$, are constant, over time, indicating that the wake structure remains in stearly rotation about, the streamwise axis, without variation in its shape and strength. $\dagger$

† The present study found that the azimuthal modes of the primitive variables remain constant over time, verifying that the wake is in steady rotation about the streamwise axis. 
(a)
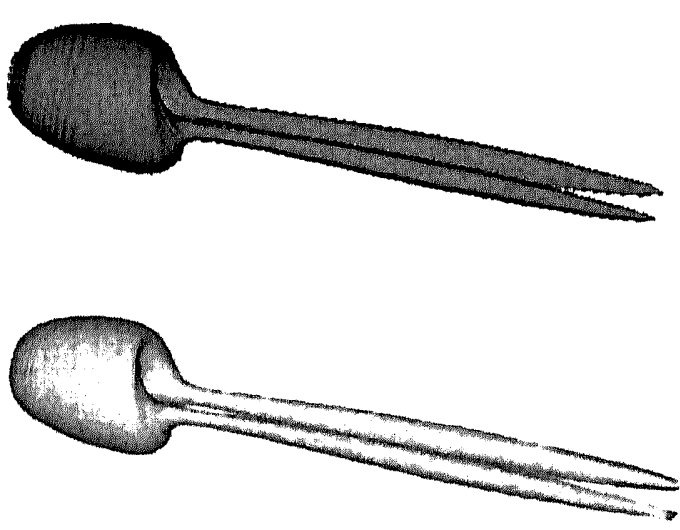

(b)
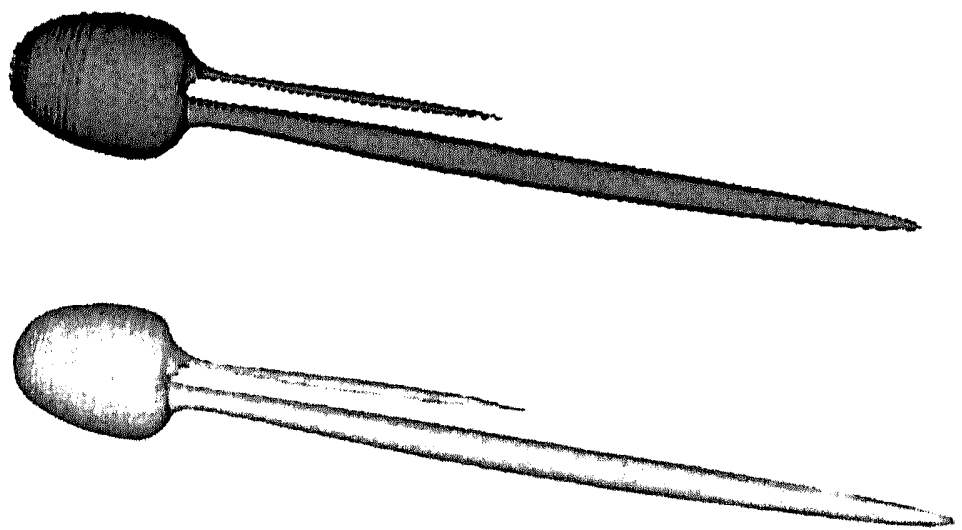

Figure 3.16: Vortical structures for $R e=250$ : (a) stationary sphere; (b) sphere rotating in the streamwise direction with $\Omega^{*}=0.10$. The structures are identified using the method of Jcong \& Hussain (1995). Dark grey structures are the results of Kim \& Choi (2002); light grey structures are the results of the presents study. $\Lambda$ threshold value of $\lambda_{2}=-8 \times 10^{-4}$ is used in the present study, while that used by Kim \& Choi is not specified. 


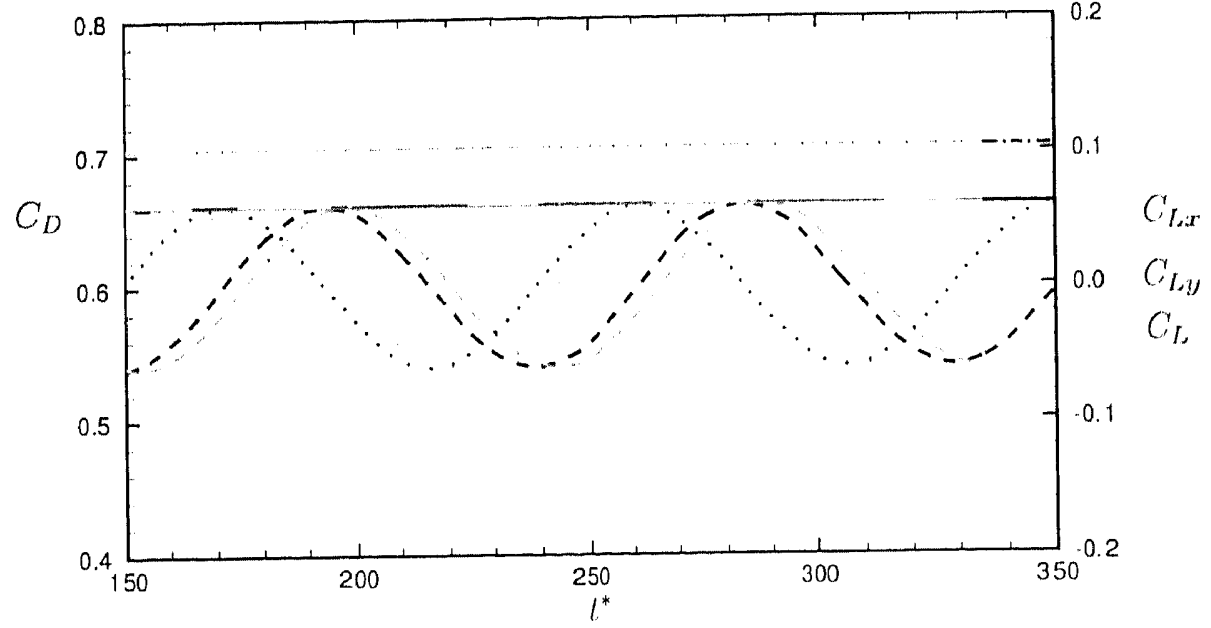

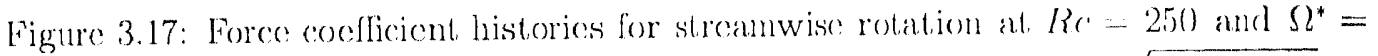

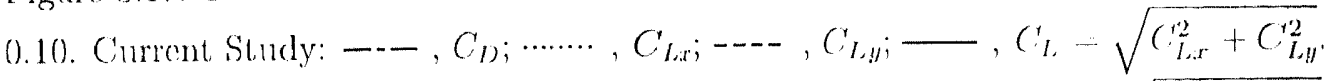

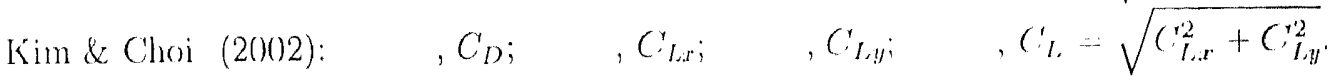




\subsubsection{Vortex shedding regime, $R e>270$}

Above $R e \approx 270$, the wake behind a stationary sphere undergoes a Hopf bifurcation to an unstcady vortex shedding regime, while a plane of symmetry remains. Figure 3.18 compares the evolution of the vortical wake over one period $(T)$ with that presented in figure 3(c) of Kim \& Choi (2002). The frame $t^{*}=T$ corresponds to an instant in time where the drag force coefficient is at a local maximum. The wake is characterized by the shedding of vortex loops, commonly referred to as "hairpin vortices." These vortices are shed from the sphere with the same orientation and with a single frequency. The: results are in close agreement with the time sequences presented by Kim \& Choi. Table 3.9 compares the force coefficients and shedding frequencies for $R e=250$ and $R e=300$ with the numorical results reported by Johnson \& Patel (1999), Kim \& Choi (2002) and Constantinescu \& Squires (2000). The result of the present study are in good agrecment with these carlicr numerical studies.

Overall, this chapter demonstrates that, over the Reynolds number range studied, the solver aceurately captures the flow physies for both a stationary and rotating sphere.

\begin{tabular}{|c|c|c|c|c|}
\hline & Re & $\overline{C_{D}}$ & $\overline{C_{L}}$ & $S t_{n}$ \\
\hline \multirow{2}{*}{ Present study } & 250 & 0.702 & 0.061 & \\
\hline & 300 & 0.658 & 0.067 & 0.134 \\
\hline \multirow{2}{*}{ Johnson \& Patcl (1999) } & 250 & & 0.062 & \\
\hline & 300 & 0.656 & 0.069 & 0.137 \\
\hline \multirow[t]{2}{*}{ Kim \& Choi (2002) } & 250 & 0.702 & 0.060 & \\
\hline & 300 & 0.657 & 0.067 & 0.134 \\
\hline \multirow[t]{2}{*}{ Constantinescu \& Squires (2000) } & 250 & 0.700 & 0.062 & \\
\hline & 300 & 0.665 & 0.065 & 0.136 \\
\hline
\end{tabular}

Table 3.9: Comparison with carlicr numcrical results of the time-averaged drag coefficient, $\overline{C_{D}}$, the lateral force coefficient, $\overline{C_{L}}$, and the Strouhal number, $S t_{n}=\int_{n} d / U_{\infty}$, where $\int_{n}$ is the frequency of vortex shedding. In this study, $f_{n}$ was taken to coincide with the dominant peak in the frequency spectra of the $C_{D}$ and $C_{L}$ time histories. 

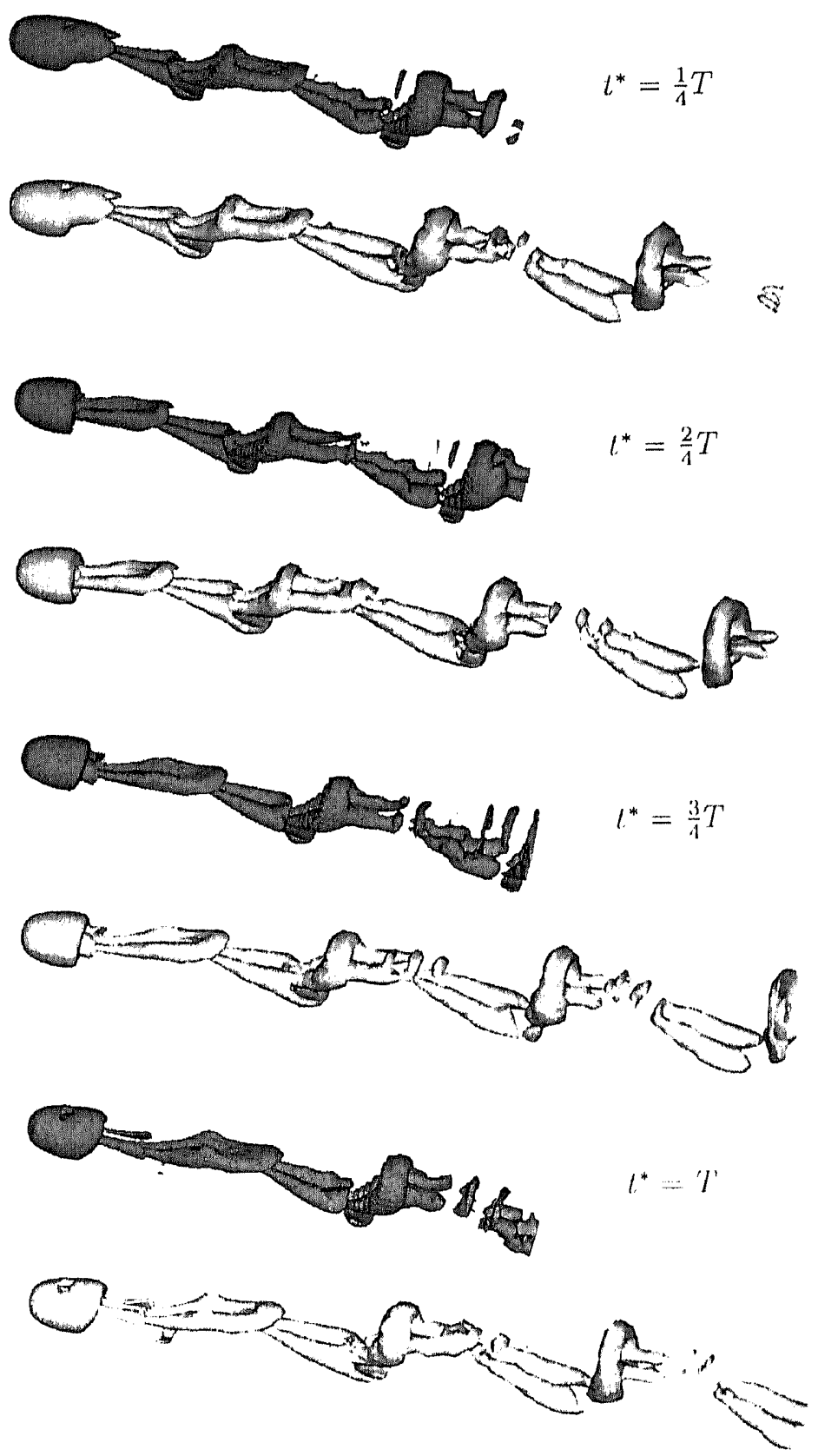

Figure 3.18: Vortical structures for a stationary sphere at, $R r=300$. The structures are identified using the method of Jeong \& Hussain (1995). Dark structures are the results of Kim \& Choi (2002); light grey structures are the results of the presents studly. A threshold value of $\lambda_{2}=-8 \times 10^{-4}$ is used in the present sturly, while that used by Kim \& Choi is not specified. 


\section{Chapter 4}

\section{Vortical structure identification}

To investigate the physics of complex fluid flow patterns it is useful to be able to identify and track vortical structures. This forms a central aspect of the current study and so the commonly used vortex identification methods and their various merits are discussed. Of the methods considered here, the $\lambda_{2}$ definition proposed by Jeong \& Hussain (1995) is shown to perform most favorably for the specific problem of the flow past, a sphere at moderate Reynolds numbers.

Unfortunately the debate on exactly what constitutes a 'vortex' remains unresolved. Robinson et al. (1989) proposed a popular qualitative definition: "A vortex exists when instantancous streamlines mapped onto a plane normal to the vortex core exhibit a roughly circular or spiral pattern, when viewed from a reference frame moving with the center of the vortex core." However, as noted by a number of anthors, this definition requires an a priori knowledge of the location of the vortex core. In a complex flowfielel, the streamlines may not complete a full revolution around a vortical structure, making vortex identification somewhat subjective.

Other intuitive indicators of vortical structures include vorticity and pressure. Vorticity magnitude, $|\omega|$, is widely used as an indicator of vortical structures, sinec there is a notion that vortical structures tend to concentrate vorticity. However, this critoria fails in a number of situations because it does not distinguish between vorticity resulting from a swirling motion (an intuitive notion of a vortex) and vorticity due to shcaring. In wall-bounded flows, or flows with ambient shear, the vorticity generated at 
the boundaries or present as a result of background shear, may be of comparable magnitude to the important vortical structures and may mask their presence. Intuitively, a vortex is often thought of as a tube-like structure consisting of a concentration of vortox lines and this has been used by Moin \& Kim (1985) to identify vorlical structures. Robinson (1991) illustrated that unless the starting point for the integration of the vorticity line lies almost precisely within the core of a vortex, the result, can be very mislcading. Again, an a priori knowledge of the location of the vortex core is required.

Elongated regions of low pressure have been widely used to educe vortical structures, with varying degrees of success. In steady two-dimensional inviscid flow, a pressure minimum occurs at the center of a vortex. The pressure minimum arises from the balance of forces between the centrifugal accelcration and the radial pressure gradiont. However, in the case of an unsteady, viscous and threc-dimensional flow, a pressure minimum does not necessarily imply the presence of a vortical structure. As noted by Jeong \& Hussain (1995), a pressure minimum may result, from an unstealy straining flow, while at low Reynolds numbers the centrifugal foree may be balanesel by viseous forces, not pressure fores. Also, the minimum of pressure inside a vortical structure depends on the surrounding pressure distribution. In flows comprising high eonecentralions of vortical structures, it is difficult to eduec a detailed desseription of the vertieces using a single pressure threshold. This measure falls shome of providing a clear picture of the vortical structures in the flow and may alse be sulbject to misinterpertation. The inadefuacies of intuitive measures for vortex ielentification ane discusserel in more detail by Joong \& Hussain (1995), Cucitore el al. (1999), Kida \& Miura (1998), Robinson (1991) and Dubief \& Delcayre (2000).

The scarch for an unambiguous definition of a vortex began to draw greater attention when it was realized that coherent vortical structures were important in many turbulent flows. A number of distinct and unambiguous definitions of a vortex have now been proposed and these have been successfully applicel to a range of problems. The discussion that follows is limited to a description of the vortex identification meth- 
ods proposed by Chong et al. (1990), Hunt et al. (1988), Jcong \& Hussain (1995) and Zhou et al. (1999). These methods are reference frame independent (or Galilean invariant) and, in essence, classify the flow locally. The methods are compared analytically by recasting their definitions in terms of the eigenvalues of the velocity gradient tensor. By expressing each criteria in terms of common kinematic parameters, the relationship between the methods is made clearer. The criteria are also applied to the flowfield obtained from the numorical simulation of the uniform flow past a sphere at $R e=300$.

\subsection{The discriminant definition of a vortex}

The flow pattern close to a point in the flow, as seen by a non-rotating observer moving with the local flow velocity, can be classified by cvaluating the eigenvalues and eigenvectors of the velocity gradient tensor,

$$
A_{i j}=\frac{\partial u_{i}}{\partial x_{j}}
$$

at that point. Here $u_{i}$ is the velocity vector and $x_{j}$ is the space vector. The velocity gradicnt, can be decomposed into symmetric and antisymmetric parts

$$
A_{i j}=S_{i j}+S_{i j}
$$

where the symmetric part is known as the rate-of-strain tensor and is given by

$$
S_{i j}=\frac{1}{2}\left(\frac{\partial u_{i}}{\partial x_{j}}+\frac{\partial u_{j}}{\partial x_{i}}\right)
$$

and the anti-symmetric part is know as the rate-of-rotation tensor and is given by

$$
\Omega_{i j}=\frac{1}{2}\left(\frac{\partial u_{i}}{\partial x_{j}}-\frac{\partial u_{j}}{\partial x_{i}}\right)
$$

For the second-order tensor $A_{i j}$, the eigenvalues $\sigma_{i}$ and associated cigenvectors $e_{i}$ are given by the eigenvalue problem

$$
\left(\mathbf{A}-\sigma_{i} I\right) \boldsymbol{e}_{i}=0
$$


The eigenvalues can be determined by solving the characteristic polynomial for $\sigma_{i}$, which takes the form

$$
\sigma_{i}^{3}+P_{A} \sigma_{i}^{2}+Q_{A} \sigma_{i}+R_{A}=0
$$

$P_{A}, Q_{A}$ and $R_{A}$ are the first, second and third invariants of $A_{i j}$, respectively. The invariants are given by

$$
\begin{aligned}
& P_{A}=-\operatorname{tr}\left(A_{i j}\right)=-S_{i i} \\
& Q_{A}=\frac{1}{2}\left(P_{A}^{2}-\operatorname{tr}\left(A_{i j}{ }^{2}\right)\right)=\frac{1}{2}\left(P_{A}^{2}-S_{i j} S_{j i}-\Omega_{i j} S_{j i}\right), \\
& R_{A}=-\operatorname{det}\left(A_{i j}\right)=\frac{1}{3}\left(-P_{A}^{3}+3 P_{A} Q_{A}-S_{i j} S_{j k} S_{k i}-3 S_{i j} \Omega_{j k} S_{k i}\right)
\end{aligned}
$$

where $\operatorname{tr}()$ and $\operatorname{det}()$ indicate the trace and the detcrminant of the tensor, respectively. For an incompressible flow, $P_{A}=0$, and the cigenvalues that govern the local topology depend only on $Q_{A}$ and $R_{A}$. The invariants of the velocily gradient tensor can be used to form the discriminant

$$
D_{A}=Q_{A}^{3}+\frac{27}{4} R_{A}^{2}
$$

Figure 4.1 presents the $\left(R_{A}, Q_{A}\right)$-plane for an incompressible flow and illustrates the four possible non-elegenerate local flow topologies. The 'lent-like' ("urve which divides characteristic ecquations that yield real and complex roots is defined by $D)_{A}=0$.

For $D_{A}>0$, cepuation (4.6) admits two complex conjugate and one real cigenvalue solutions. Points which admit such solutions are satid to be focal in nature. In this (atse, a focus is contained in the plane delined by the cigenvectors comresponding to the complex cigenvalues and solution trajectories wrap around the axis of the real eigenvector. The direction that the fluid particle follows is determined by the sign of the third invariant, $R_{A}$. If $R_{A}>0$, the flow moves away from the center of the local critical point and mass conscrvation dictates that it must move towards the axis of the real cigenvector. It is said to be unstable focus/contracting (UF/C) according to the terminology of Chong et al. (1990). For $R_{A}<0$, the flow direction is reversed and 


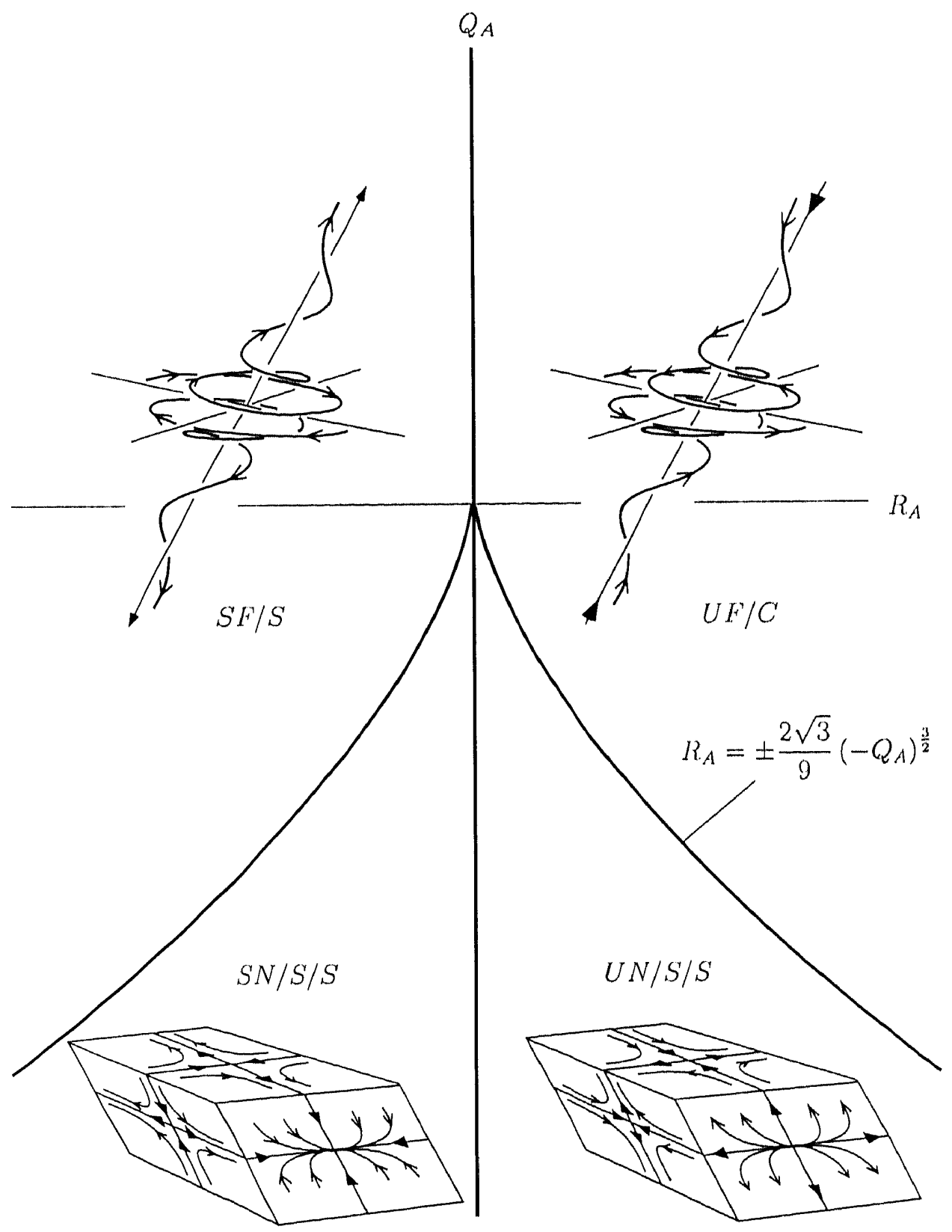

Figure 4.1: Local non-degenerate topologics for incompressible flows. SF/S: stable focus/stretching, UF/C: unstable focus/contracting, SN/S/S: stable node/saddle/saddle and USN/S/S: unstable node/saddle/saddle. 
the topology is described as stable focus/stretching $(\mathrm{SF} / \mathrm{S}) . \dagger$ For $D_{A}<0$, all three cigenvalues are real and the local flow is strain dominated. For $R_{A}<0$, the local topology is referred to as stable/node/saddle/saddle (SN/S/S) and for $R_{A}>0$ it is referred to as unstable node/saddle/saddle (UN/S/S). For $D_{A}=0$, the cigenvalues are all real and equal. The degenerate case of $R_{A}=0$ corresponds to two-dimensional flow. Using this 'vocabulary', the invariants and the discriminant can be used to classify the flow at cach point in a flowficld.

\subsection{The $Q_{A}$ definition of a vortex}

From equation (4.8), the second invariant may also be written as

$$
Q_{A}=\frac{1}{2}\left(\Omega_{i j} \Omega_{i j}-S_{i j} S_{i j}\right)
$$

$S_{i j} S_{i j}$ gives a measure of the strength of irrotalional stretching, ancl $S_{i j} \Omega_{i, j}$ is a measure of the local strength of the vorticity. Both $S_{i j} S_{i j}$ and $\Omega_{i j} \Omega_{i j}$ are pesitive scalar quantities, therefore the invariant, $Q_{A}$ may be thought, of as the local balance between the strength of rotation over the strain. Hunt el al. (1988) defincel a vortex as a region with positive second invariant, with the added condition that the pressure must be: less than ambient. This is known as the $Q_{A}$ critcrial of vortex irfentification. Here we define the $Q_{A}$ critcria without the adeded constraint hat the pressume must be less than ambient. From figure 4.1 it is clear that the $Q_{A}$ criteria is, in grencral, more restrivetive than the $D_{A}$ criteria. $Q_{A}$ is directly related to the Laplacian of pressince by

$$
Q_{A}=-\frac{1}{2} \frac{\partial u_{i}}{\partial x_{j}} \frac{\partial u_{j}}{\partial x_{i}}=\frac{1}{2 \rho} \nabla^{2} I^{\prime}
$$

Earlier it was noted that the pressure inside a vortical structure depends on the surrounding pressure and so it can be difficult to capture the structure of many vortices using a single pressure level. As noted by Kida \& Miura (1998), the Laplacien of the

$\dagger$ Chakraborty et al. (2004) state that the $D_{A}$ criteria does not clistiuguish between inward and outward spiralling motion. However, it is well-known that the sign of $R_{A}$ demarcates the local flow as either stable focus/stretching or unstable focus/contracting. 
pressure is free from this problem, while still being able to capture the low pressure regions in the flow.

\subsection{The $\lambda_{2}$ definition of a vortex}

Jeong \& Hussain (1995) introduced the $\lambda_{2}$ definition of a vortex by considering the inadequacies of the pressure minimum criteria for vortex identification. They noted that the pressure critcria fails because: $(a)$ an unsteady strain can lead to a pressure minimum, and $(b)$ the centrifugal acceleration may be balanced by viscous forces, which can climinale a pressure minimum in a vortical flow. The $\lambda_{2}$ definition corresponds to a pressure minimum in a plane, after eliminating the contributions of unsteady straining and viscous terms in the Navier Stokes cquations.

In conservalive tensor form, the incompressible Navier Stokes equations can be written as

$$
\frac{\partial u_{i}}{\partial t}+\frac{\partial}{\partial x_{k}}\left(u_{k} u_{i}\right)=-\frac{1}{\rho} \frac{\partial P}{\partial x_{i}}+\nu \frac{\partial^{2} u_{i}}{\partial x_{k} x_{k}}
$$

Taking the gradient of equation (4.13) leads to the following equation for the covolution of the velocity gradient tensor

$$
\frac{D}{D t}\left(\frac{\partial u_{i}}{\partial x_{j}}\right)+\frac{\partial u_{k}}{\partial x_{j}} \frac{\partial u_{i}}{\partial x_{k}}=-\frac{1}{\rho} \frac{\partial^{2} P}{\partial x_{i} \partial x_{j}}+\nu \frac{\partial^{3} u_{i}}{\partial x_{j} \partial x_{k} \partial x_{k}}
$$

where

$$
\frac{D}{D t}=\frac{\partial}{\partial t}+u_{k} \frac{\partial}{\partial x_{k}}
$$

Decomposing the velocity gradient tensor into symmetric and anti-symmetric parts, equation (1.14) becomes

$$
\frac{D}{D \iota}\left(S_{i j}+\Omega_{i j}\right)+\left(S_{k j}+S_{k j}\right)\left(S_{i k}+\Omega_{i k}\right)=-\frac{1}{\rho} \frac{\partial^{2} P}{\partial x_{i} \partial x_{j}}+\nu \frac{\partial^{2}}{\partial x_{k} \partial x_{k}}\left(S_{i j}+\Omega_{i j}\right)
$$

The symmetric part of this equation is

$$
\frac{D S_{i j}}{D t}-\nu \frac{\partial^{2} S_{i j}}{\partial x_{k} \partial x_{k}}+S_{k j} S_{i k}+\Omega_{k j} \Omega_{i k}=-\frac{1}{\rho} \frac{\partial^{2} P}{\partial x_{i} \partial x_{j}} .
$$


Here $\frac{\partial^{2} P}{\partial x_{i} \partial x_{j}}$ is the Hessian of pressure and contains information about the local pressure extrema. The existence of a pressure minimum requires two positive eigenvalues of the pressure Hessian tensor, the corresponding eigenvectors define the plane on which the pressure minimum lies. In cquation (4.17), the first term represents unsteady strain and the second term comprises viscous effects. By ignoring these contributions to a pressure minimum, Jcong \& Hussain defined a vortex as a connected region with two negative cigenvalues of the tensor $S^{2}+\Omega^{2}$. $S^{2}+\Omega^{2}$ is symmetric and has the real cigenvalues $\lambda_{1}, \lambda_{2}$ and $\lambda_{3}$. If the cigenvalues are ordered, such that $\lambda_{1} \geq \lambda_{2} \geq \lambda_{3}$, the definition is cquivalent to

$$
\lambda_{2}<0
$$

\subsection{The swirling strength definition of a vortex}

Zhou et al. (1999) used the critical point classification of Chong cet al. (1990) and defined vortical structures in the flow as having complex eigenvalues of the velecily gradient tensor. In canonical form, the velocily gradiont, tensor can be expressed at

$$
\mathbf{A}^{\prime}=\left[\begin{array}{ccc}
\sigma_{\mathrm{cr}} & -\sigma_{\mathrm{ci}} & 0 \\
\sigma_{\mathrm{ci}} & \sigma_{\mathrm{cr}} & 0 \\
0 & 0 & -2 \sigma_{\mathrm{cr} \mathrm{r}}
\end{array}\right]
$$

where the cigenvalues are

$$
\begin{array}{r}
\sigma_{1,2}=\sigma_{\mathrm{cr}} \pm \mathrm{i} \sigma_{\mathrm{ci}}, \\
\sigma_{3}=-2 \sigma_{\mathrm{cr}} .
\end{array}
$$

In a coordinate system defined by the eigenvectors corresponding to these cigenvalues, the equations describing the streamlines in the neighborhosed of the critical point may be expressed as

$$
\begin{aligned}
& x_{1}(t)=e^{\sigma_{\mathrm{cr}} t}\left\{C_{1} \cos \sigma_{\mathrm{ci}} t-C_{2} \sin \sigma_{\mathrm{ci}} t\right\} \\
& x_{2}(t)=e^{\sigma_{\mathrm{cr}} t}\left\{C_{2} \cos \sigma_{\mathrm{ci}} t+C_{1} \sin \sigma_{\mathrm{ci}} t\right\} \\
& x_{3}(t)=C_{3} e^{-2 \sigma_{\mathrm{cr}} t} .
\end{aligned}
$$


$C_{1}, C_{2}$ and $C_{3}$ are constants and $t$ denotes a pseudo-time that cvolves the solution trajectories. These equations describe the streamline pattern illustrated in figure 4.1 for $D_{A}>0$. The flow is stretched or compressed along the $x_{3}$-axis, while the rate at which the streamlines spiral around the $x_{3}$-axis is determined by the magnitude of the imaginary part of the complex eigenvalues, $\left|\sigma_{\mathrm{ci}}\right|$. Zhou et al. (1999) used $\sigma_{\text {ci }}^{2}$ to quantify the strength of the local swirling motion, which they defined as the swirling strength of a vortex.

\subsection{A critical evaluation of the vortex identification methods}

The vortex identification criteria described in the previous sections can be compared analytically by recasting their definitions in terms of the cigenvalues of the velocity gradient tensor, A. Provided that $\mathbf{A}$ is non-singular, there always exists a matrix $\mathbf{B}$, that transforms the velocity gradient tensor into the canonical form, $\mathbf{A}^{\prime}$, though the operation

$$
\mathbf{A}^{\prime}=\mathbf{B A B}^{-1}
$$

The tensors $\mathbf{A}$ and $\mathbf{A}^{\prime}$ have the same eigenvalues, therefore it, is convenient to consider the canonical form to simplify the analysis. As already mention in $\$ 4.4$, for complex cigenvalues, $\mathbf{A}^{\prime}$ is given by

$$
\mathbf{A}^{\prime}=\left[\begin{array}{ccc}
\sigma_{\mathrm{cr}} & -\sigma_{\mathrm{ci}} & 0 \\
\sigma_{\mathrm{ci}} & \sigma_{\mathrm{cr}} & 0 \\
0 & 0 & -2 \sigma_{\mathrm{cr}}
\end{array}\right] .
$$

In this case, equation (4.10) for the discriminant, may be re-expressed as

$$
D_{A}=\sigma_{\mathrm{ci}}^{6}\left[1+9\left(\frac{\sigma_{\mathrm{cr}}}{\sigma_{\mathrm{cli}}}\right)^{2}\right]^{2}>0 .
$$

Equation (4.11) for the second invariant of the velocity gradient tensor, may be reexpressed as

$$
Q_{A}=\sigma_{\mathrm{ci}}^{2}\left[1-3\left(\frac{\sigma_{\mathrm{cr}}}{\sigma_{\mathrm{ci}}}\right)^{2}\right]>0 .
$$


and the swirl strength definition may be expressed as

$$
\sigma_{\mathrm{ci}}^{2}>0
$$

In gencral, the $\lambda_{2}$ definition cannot be expressed solely in terms of the eigenvalues of $A_{i j} . \lambda_{2}$ also depends on the orientation of the eigenvectors of $A_{i j}$. In order to explore the gencral relationship between $\lambda_{2}$ and the cigenvalues of $A_{i j}$, Chakraborty et al. (2004) found it necessary to consider all possible configurations of $A_{i j}$. However, in the canonical case, where the eigenvector planes are orthogonal, $\lambda_{2}$ can be expressed uniquely in terms of cigenvalues of $A_{i j}$ as

$$
\lambda_{2}=\sigma_{\mathrm{ci}}^{2}\left[\left(\frac{\sigma_{\mathrm{cr}}}{\sigma_{\mathrm{ci}}}\right)^{2}-1\right]<0 .
$$

For the $D_{A}$ and swirl strength criteria, all combinations of $\left|\sigma_{c \mathrm{i}}\right|>0$ and the ratio $\left|\sigma_{\mathrm{cr}} / \sigma_{\mathrm{ci}}\right|$ class the local flow as vortical. Using the nomenclature of Chong et al. (1990), the sign of $\sigma_{\text {cr }}$ determines whether the solution trajectories form a slable focus $\left(\sigma_{c r}<0\right)$ or unstable focus $\left(\sigma_{\mathrm{cr}}>0\right)$. The ratio $\left|\sigma_{\mathrm{cr}} / \sigma_{\mathrm{ci}}\right|$ is a measure of the compactuess of the spiral trajectory. For large $\left|\sigma_{\mathrm{cr}} / \sigma_{\mathrm{ci}}\right|$, the focus rapielly spirals away, or towards the eigenvector plane formed by the complex eigenvectors, while for small $\left|\sigma_{c r} / \sigma_{c i}\right|$, the focus forms a compact structure. Figure 4.2 presents t,wo examples of a focus with cqual $\sigma_{\mathrm{ci}}$ and different $\left|\sigma_{\mathrm{cr}} / \sigma_{\mathrm{ci}}\right|$. The intuitive notion of a vortex is consistent, with the structure presented in figure 4.2 (a), while the structure presented in figure 4.2 (b) appears to be a less significant vortical element the $D_{A}$ and swirl strength criteria (lo not make a distinction between the two cases. In fact, for the I) a criteria, the case of higher $\left|\sigma_{\mathrm{cr}} / \sigma_{\mathrm{ci}}\right|$ will give a higher magnitude of $D_{A}$ and so will register as being a stronger vorlical structure.

The $Q_{A}$ and $\lambda_{2}$ definitions impose bounds on $\left|\sigma_{\mathrm{cr}} / \sigma_{\mathrm{ci}}\right|$, out side which, the local flow is no longer considered to form part of a vortical structure, even though $\left|\sigma_{c i}\right|>0$. For the $Q_{A}$ definition, the local flow qualifies as part of a vortical structure when

$$
\left|\frac{\sigma_{\mathrm{cr}}}{\sigma_{\mathrm{ci}}}\right|<\frac{1}{\sqrt{3}}
$$


$(a)$

(b)

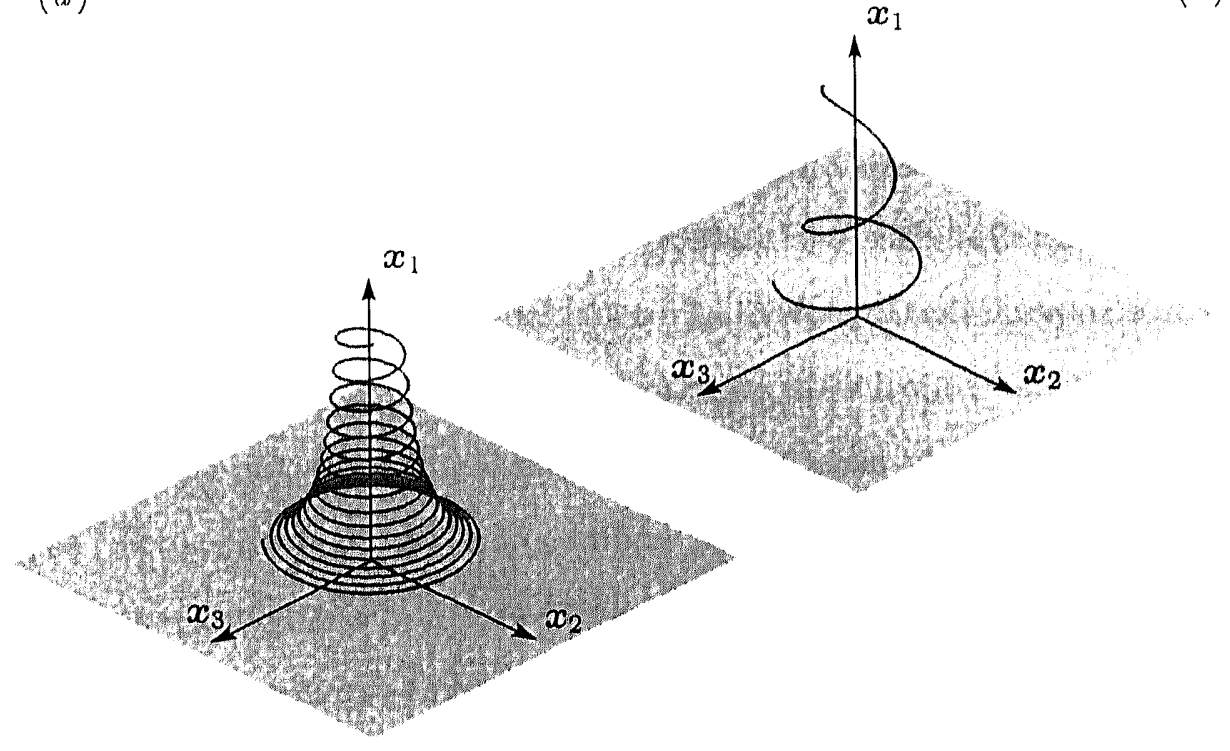

Figure 4.2: Streamline pattern in the vicinity of a canonical eritical point. The swirl strength is fixed $\left(\sigma_{\mathrm{ci}}=0.8\right)$ and $\sigma_{\mathrm{cr}}$ varics: $(a) \sigma_{\mathrm{cr}}=-0.01 ;(b) \sigma_{\mathrm{cr}}=-0.06$.

For the special cases of orthogonal eigenvector planes, the $\lambda_{2}$ definition classifics the local flow as vortical for

$$
\left|\frac{\sigma_{\mathrm{cr}}}{\sigma_{\mathrm{ci}}}\right|<1
$$

In the general case where the eigenvector planes are not orthogonal, Chakraborty et al. $(2004)$ find that $\lambda_{2}=0$ is approximately bounded by

$$
\left|\frac{\sigma_{\mathrm{cr}}}{\sigma_{\mathrm{ci}}}\right|<1.3
$$

In summary, the $D_{A}, Q_{A}, \lambda_{2}$ and swirling strength critcria all define a vortex as having complex eigenvalues of $A_{i j}$, but impose different bounds on $\left|\sigma_{\mathrm{cr}} / \sigma_{\mathrm{ci}}\right|$. In light of this, it is expected that the methods should only show significant differences in regions of the flow where $\left|\sigma_{\mathrm{cr}} / \sigma_{\mathrm{ci}}\right|$ is large. 


\subsubsection{Comparative application to the flow past a sphere}

The different identification criteria are compared in figure 4.3 to figure 4.5 for the uniform flow past a stationary sphere at $R e=300$. The flow structure is shown at an instant in time where the magnitude of the lateral force coefficiont is at a local maximum. Figure $4.3(a-c)$ presents iso-surfaces of streamwisc vorticity, vorticity magnitude and $Q_{A}$. Because vorticity cannot distinguish between rotation duc to shear and rotation due to a swirling motion, the vorticity magnitude fails to capture the details of the vortical wake structure. Also, the geometric structure educed using iso-surfaces of vorticity magnitude varies considerably with the choice of threshold, which makes it a poor candidate for unambiguous vortex identification. Iso-surfaces of strcamwise vorticity educe more clearly the streamwise structures in the wake, but do not provide a complete picture. The iso-surfaces capture the shear layer which scparates from the sphere surface. This obscures the tubular vortical structures in the near-wake. Further downstream, the presence of the shear is less felt, and the vortical structures are more discernable. Since the $Q_{A}$ criteria identifies only regions where the vorticity magnitude prevails over the strain, it is able to extract the more dynamically significant vortical structures in the wake.

Figure $4.4(a)$ and figure $4.4(b)$ present, iso-surfaces of the swirl strength, $\sigma_{c \mathrm{i}}^{2}$, and of $D_{A}$. For the $D_{A}$ criteria, the iso-surfaces have been divided into regions which comprise stable focus/stretching and unstable focus/contracting topologies. Figure 4.5 (a c) presents iso-surfaces of $\lambda_{2}$ for three threshold values. Similar structures are educed using the $Q_{A}$ and $\lambda_{2}$ criteria. Close agrecment between the methods has also been obscrved by Dubief \& Deleayre (2000), for a number of different turbulent flows. The structures educed using the $D_{A}$ and swirling strength criteria are also very similar. The most striking difference is that the $D_{A}$ criteria captures a bulb-shaped structure dircetly upstream of the sphere, where the local flow topology is unstable focus/contracting. Figure 4.4 (c) presents iso-surfaces of $\left|\sigma_{\mathrm{cr}} / \sigma_{\mathrm{ci}}\right|=1$, in regions of the flow where $D_{A}>1 \times 10^{-6}$. Upstream of the sphere these is a region of large 
$\left|\sigma_{\mathrm{ci}} / \sigma_{\mathrm{cr}}\right|$, which coincides with a similar structure seen in the iso-surfaces of $D_{A}$. As mentioned carlicr, equation (4.26) docs not impose bounds on $\left|\sigma_{\mathrm{cr}} / \sigma_{\mathrm{ci}}\right|$, the result of which is the bulb structure upstream of the sphere. This is clearly not an important vortical structure, but an artifact of the identification method. Jeong \& Hussain (1995) noted that vortical structures identified using $D_{A}$ tend to have noisy boundarics. The lack of bounds on $\left|\sigma_{\mathrm{cr}} / \sigma_{\mathrm{ci}}\right|$ for this method appears to contribute to this noise. In the wake $\left|\sigma_{\mathrm{er}} / \sigma_{\mathrm{ei}}\right|$ has moderate values, which explains the similarity in the wake structures educed by the different criteria.

Although the $D_{A}, Q_{A}, \lambda_{2}$ and swirling strength eriteria define the boundary of a vortical structure for zero threshold, a small non-zero threshold has been used in cach case. This minimizes numerical noise and isolates the more intense vortical structures, which are expected to be more dynamically significant. An attempt has been made to choose thresholds which give comparable diameter structures from the different methods. An important property of a vortex identification criteria is that it should be insensitive to the threshold value used for the visualization. For the test case considered here, the $\lambda_{2}$ criteria performed most favorably in this regard. Figure $4.5(a c)$ presents isc-surfaces of $\lambda_{2}=-8 \times 10^{-3},-8 \times 10^{-4}$ and $-8 \times 10^{-6}$. Although the threshold values have a range of threc orders of magnitude, very similar structures are educed. For thresholds larger than $\lambda_{2}=-8 \times 10^{-3}$, the diameters and the spatial extent of the wake structures is reduced, while for thresholds smaller than $-8 \times 10^{-6}$, the dianeters of the structures grow such that they begin to merge and the characteristics of the wake are obscured.

Based on the findings presented here, the $\lambda_{2}$ criteria is used in subsequent chapters to efliciently capture the characteristics of the wake. Although the $Q_{A}$ and $\lambda_{2}$ criteria chluce similar structures, the $\lambda_{2}$ is preferred, sinec for the specific problem of the flow past a sphere at moderate Reynolds number, it is less sensitive to the threshold value used. 


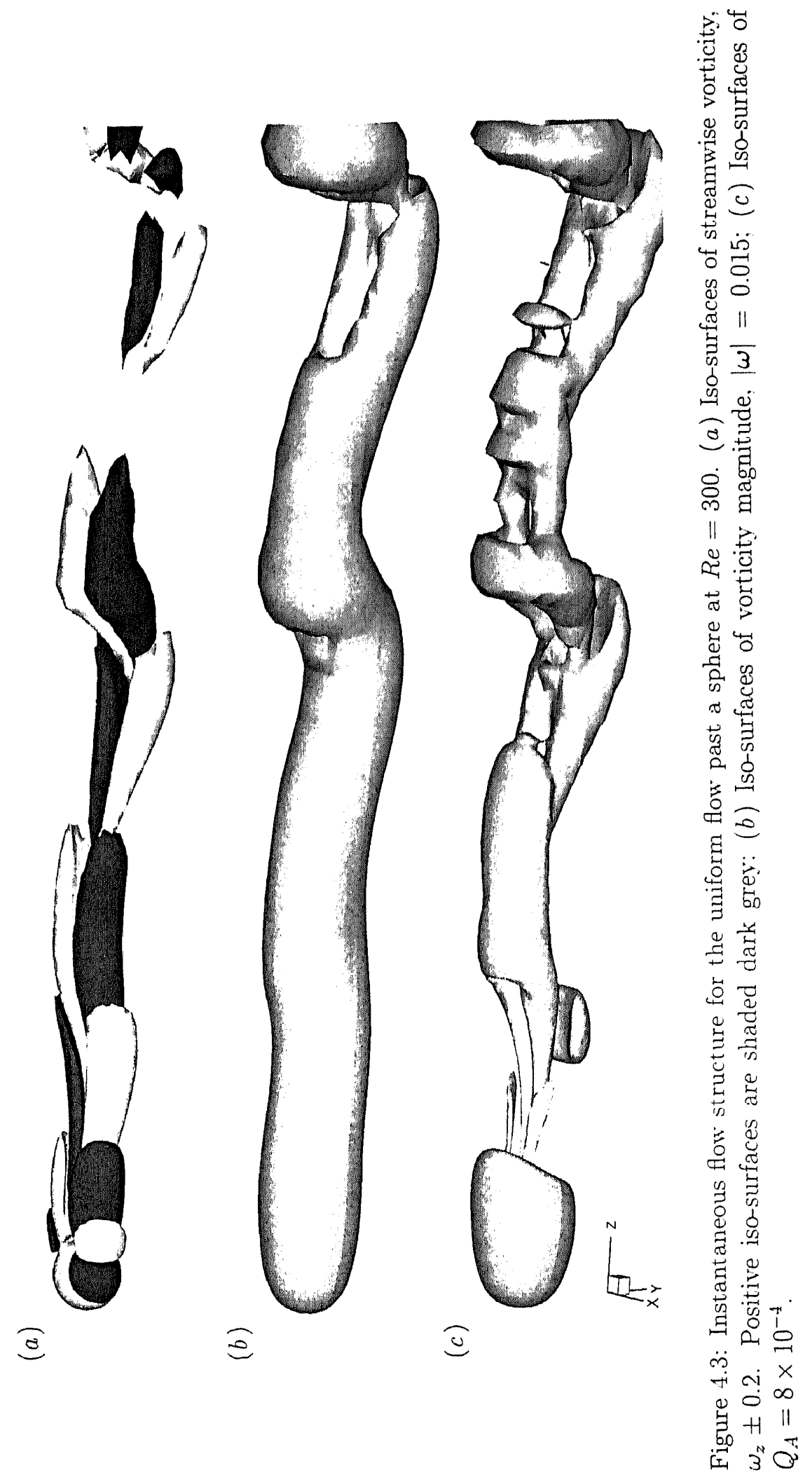




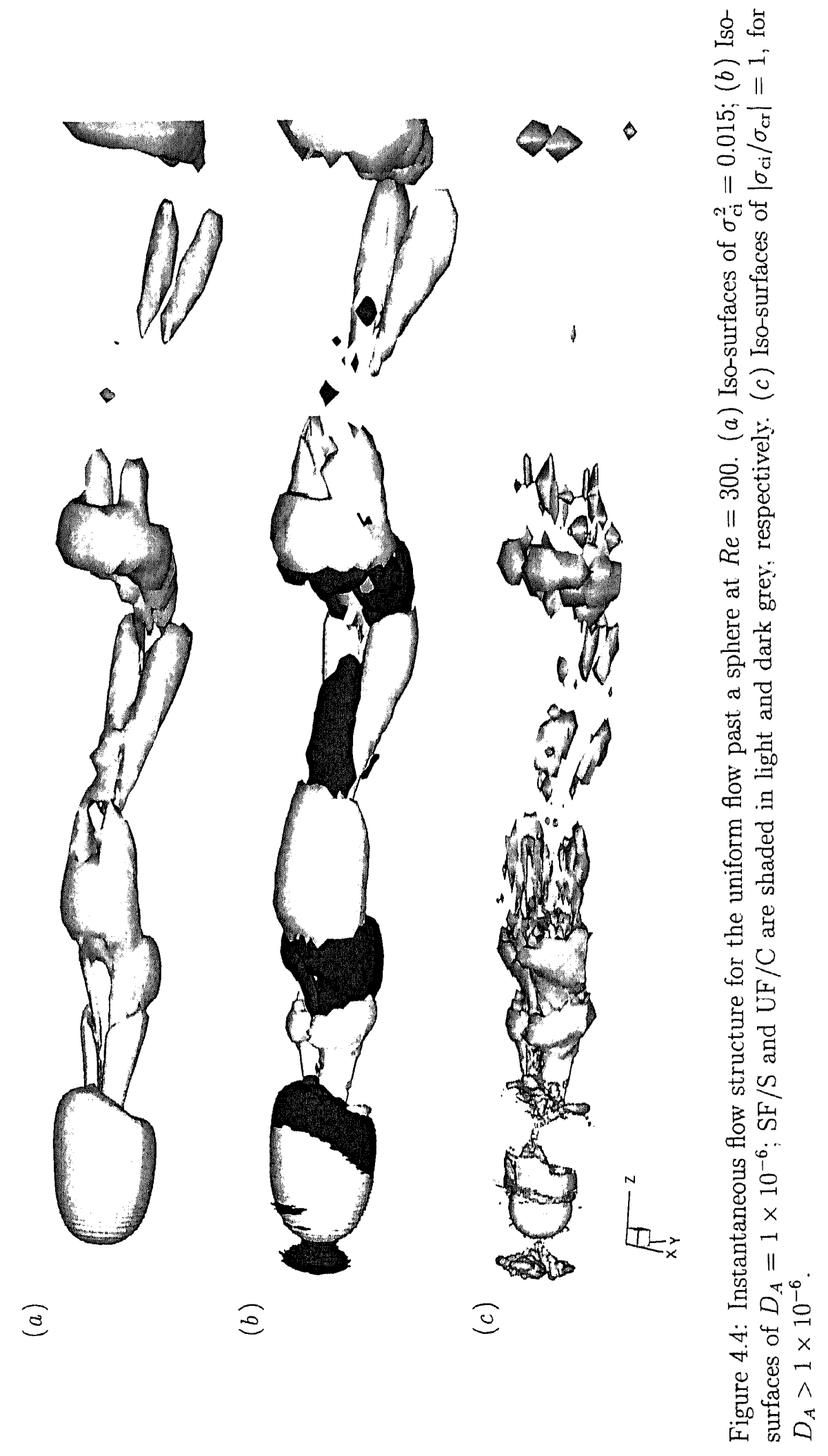




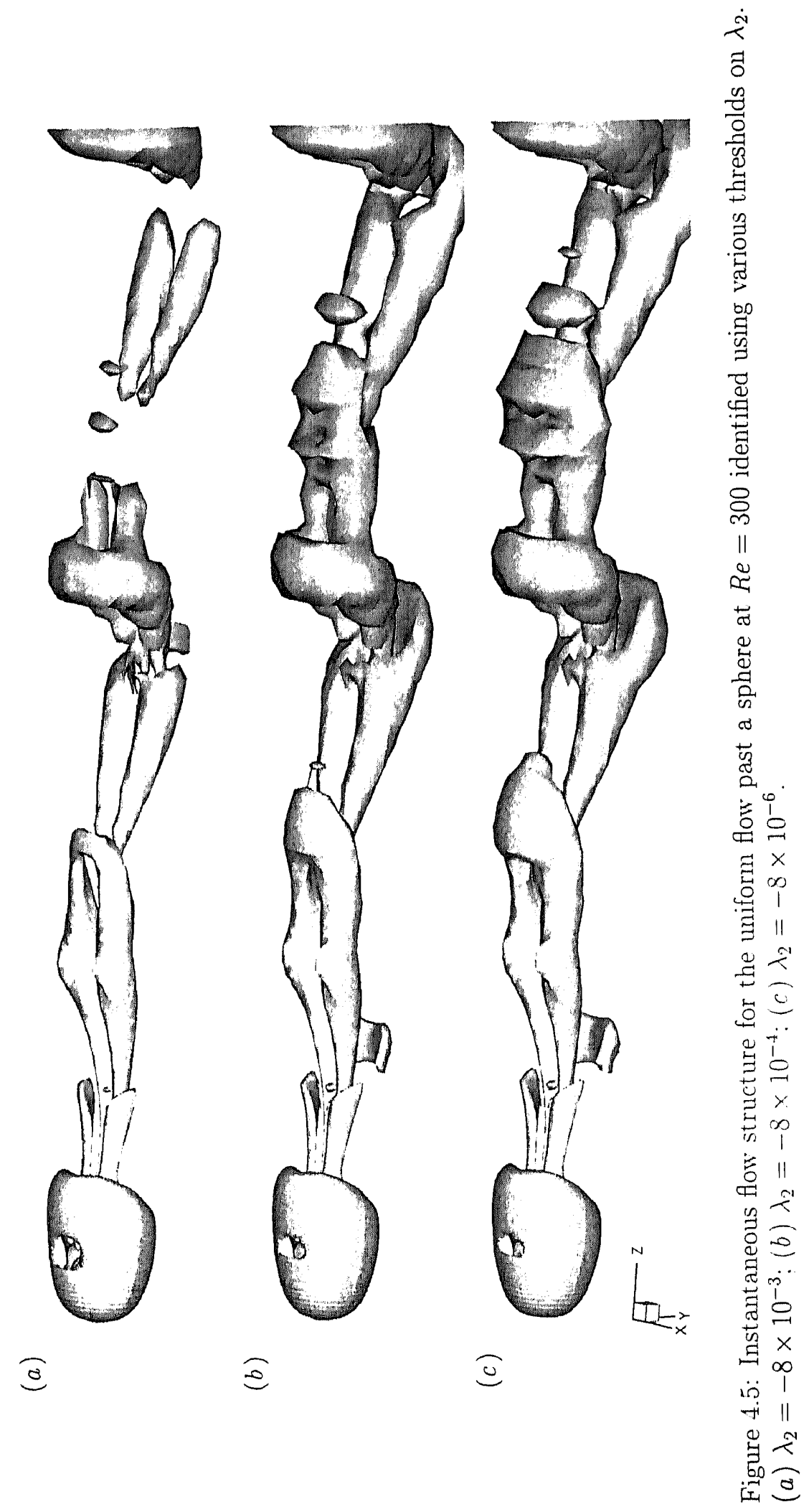




\section{Chapter 5}

\section{Flow past a sphere undergoing steady transverse rotation}

This chapter presents results from the numerical simulation of a transversely spiming sphere at $R e=100,250$ and 300 and non-dimensional rotation rates in the range $0.00 \leq \Omega^{*} \leq 1.00$. For a stationary sphere, the Reynolds numbers 100,250 and 300 cover the axisymmetric, asymmetric steady and vortex shedding regimes, respectively. These Reynolds numbers were also chosen because they have been considered in a number of carlicr numerical studies for a stationary sphere and a sphere in streanwise: and transverse rotation, thus allowing the simulations to be validated.

The range of $\Omega^{*}$ was established by numerical experiment. The discrete values considered vary with Reynolds number and are listed in table 5.1. Their choice was tailored to highlight the significant effects of sphere rotation and also to allow some direct comparison with carlicr studies. Owing to computational constraints, the rotation rate was linited to $\Omega^{*} \leq 1.00$. Simulations at higher rotation rates became marginally resolved and an adequate grid sensitivity study was not feasible. In all cases, insensitivity to spatial and temporal discretization were verified. Examples of the validation procedure are given in chapter 3. Also listed in table 5.1 are the number of azimuthal collocation points, $N_{\phi}$, used to ensure a grid independent solution. The other important spatial and temporal discretization parameters were not varied between the different cases and are listed in table 5.2 . 


\begin{tabular}{cc|cc|cc}
\hline \multicolumn{2}{c|}{$R e=100$} & \multicolumn{2}{c|}{$R e=250$} & \multicolumn{2}{c}{$R e=300$} \\
\hline$\Omega^{*}$ & $N_{\phi}$ & $\Omega^{*}$ & $N_{\phi}$ & $\Omega^{*}$ & $N_{\phi}$ \\
\hline 0.00 & 16 & 0.00 & 16 & 0.00 & 32 \\
0.025 & 16 & 0.05 & 32 & 0.05 & 32 \\
0.05 & 16 & 0.08 & 32 & 0.10 & 64 \\
0.10 & 16 & 0.10 & 32 & 0.25 & 64 \\
0.25 & 16 & 0.20 & 64 & 0.30 & 64 \\
0.30 & 16 & 0.25 & 64 & 0.50 & 64 \\
0.50 & 16 & 0.30 & 64 & 0.60 & 64 \\
0.60 & 16 & 0.50 & 32 & 0.80 & 64 \\
0.80 & 32 & 0.60 & 32 & 1.00 & 64 \\
1.00 & 32 & 0.80 & 64 & & \\
& & 1.00 & 64 & & \\
\hline
\end{tabular}

Table 5.1: Discrete values of non-dimensional rotation rate simulated.

\begin{tabular}{cccccccc}
\hline$N_{r}$ & $C_{0}$ & $x_{\min }$ & $N_{\theta}$ & $\Theta$ & $\Delta t^{*}$ & $d$ & $D_{\circ}$ \\
\hline 121 & -0.6 & 0.3 & 100 & -0.35 & 0.0005 & 1.0 & 22.5 \\
\hline
\end{tabular}

Table 5.2: Spatial and temporal simulation parametcrs.

The objective of this chapter is to illustrate the various wake transitions that take? place with increasing rotation rate. An attempt is also made to describe the underlying mechanisms. In $\$ 5.1$ the vortical wake structure, as identified using the Jeong \& Hussain (1995) $\lambda_{2}$ definition, is presented. To provide further insight into the nature of the transitions that are observed, $\S 5.2$ presents the near-wake streamline patterns, \$5.3 the vorticity ficld and $\$ 5.4$ the pressure field. It is hoped that these supporting sections combine to provide a better understandling of the mechanisms that shape the wake structures presented in $\$ 5.1$. For the numerical simulation of particulate llows, the forces and moments experienced by a spinning sphere are of interest. These are presented in $§ 5.5$. Finally the important findings of the chapter are summarized in $\S 5.6$. 


\subsection{Vortical wake structure}

In this section the wake structure is examined using the vortex identification method of Jcong \& Hussain (1995). In all cases a threshold value of $\lambda_{2}=-8 \times 10^{-4}$ is used. This choice of threshold value was justified in chapter 4 . To indicate length scale, the wake cross-sections are marked at streamwise increments of $\Delta z=2$, starting at $z=0$.

Figure 5.1 presents the flow structure at $R e=100$ for a number of representative rotation rates. At $R e=100$ the wake remains steady over the range of $\Omega^{*}$ considered. For a stationary sphere, the flow topology comprises a shroud over the sphere and a torus in the near-wake. This wake flow is described in some detail by Johnson \& Patel (1999). The torus in the near-wake coincides with the axisymmetrie toroidal wake found at this Reynolds number, while, as noted by Johnson \& Patel, the shroud over the sphere does not coincide with any apparent vortical structure. However, they showed that in a coordinate system translating with the local velocity, closed stroamline orbits coincide with the position of the shroud.

The introduction of sphere rotation forces the wake to undergo a transition to a clouble-thread structure, similar to that found for a stationary sphore above the regular transition $(212<R e<270)$. For $\Omega^{*}=0.05$, the double-threads exist but are comparatively weak and are not detected at the throshold value of $\lambda_{2}$ presented. Sphere rotation also distorts the shroud enveloping the sphere, causing it to grow over the advancing side and diminish over the retreating side of the sphere. The toroidal structure in the near-wake becomes progressively tilted and distorted.

With increasing $\Omega^{*}$, the threads become thicker and elongated in the streanwise dircetion indicating an increase in their rotational strength. The threads are also deflected in the negative $y$-direction as a result of the induced velocity of one tail on the other. These quasi-streamwise structures show some similarity to the vortices which trail a finite length wing. Here an analogy can be made between the rotation rate of the sphere and the angle of attack of the wing, which as it is increased, leacls 
to a higher lift coefficient and the intensification of the quasi-streamwise structures in the wake.

The effect of sphere rotation on the flow structure at $R c=250$ is illustrated in figure 5.2. For a stationary sphere, the wake comprises a double-threarl structure, which has becn previously observed through both experiment and numerical simulation. As will be shown in $\S 5.3$, these tails consist of strcamwisc vorticity, equal in magnitude and opposite in sign. It is through these tails that fluid entrained into the near-wake is released downstream. The introduction of sphere rotation causes the tails to become clongated in the streamwise direction, as was also obscrved for $R e=100$. However, above a critical value of the rotation rate, the wake undergoes a transition to an unsteady vortex shedding regime. This transition occurs at approximatcly $\Omega^{*}=0.08$. Simulation at this non-dimensional rotation rate show small amplitude wake oscillations with a slow decay rate, indicating that the wake is in the vicinity of a bifurcation between as steady and unstcady regime. In figure 5.2 the instantaneous wake structure is presented for $t^{*}=256.15$. The simulation was continued $\left.t, 0 l^{*}=6(0) .00\right)$, by which time the wake settles to a steady double-thread. At higher rolation rates, the wake: undergoes sustained vortex shedding. In these and subsecyuent figures that present, a periodic wake, unless otherwise noted, the time instant shown coincides with the latcral lift force being at a local minimum. With increasing $\Omega^{*}$, the lateral expousion of the wake increases before decreasing again, such that for $\Omega^{*} \geq 0.30$, vorlex shedeling is suppressed and the wake reverts to the double-thread structure. The lateral expansion and contraction of the wake coincides with the increase and decrease in the oscillating amplitude in the force histories presented in $\$ 5.5$. Some waviness is seen in the streamwise threads for $\Omega^{*}=0.30$, while the wake is stcady at higher $\Omega^{*}$.

For cases $\Omega^{*}=0.10$ and 0.20 , a time sequence of the wake evolution is presented in figures 5.3 and 5.4, respectively. Starting at the time instant where the lift force is at a local minimum, denoted as $t_{o}^{*}$, the wake structure is presented at every quarter period, for one period $(T)$. Here, the time variable $t_{c}^{*}=t^{*}-t_{o}^{*}$ is introduced for convenience. 


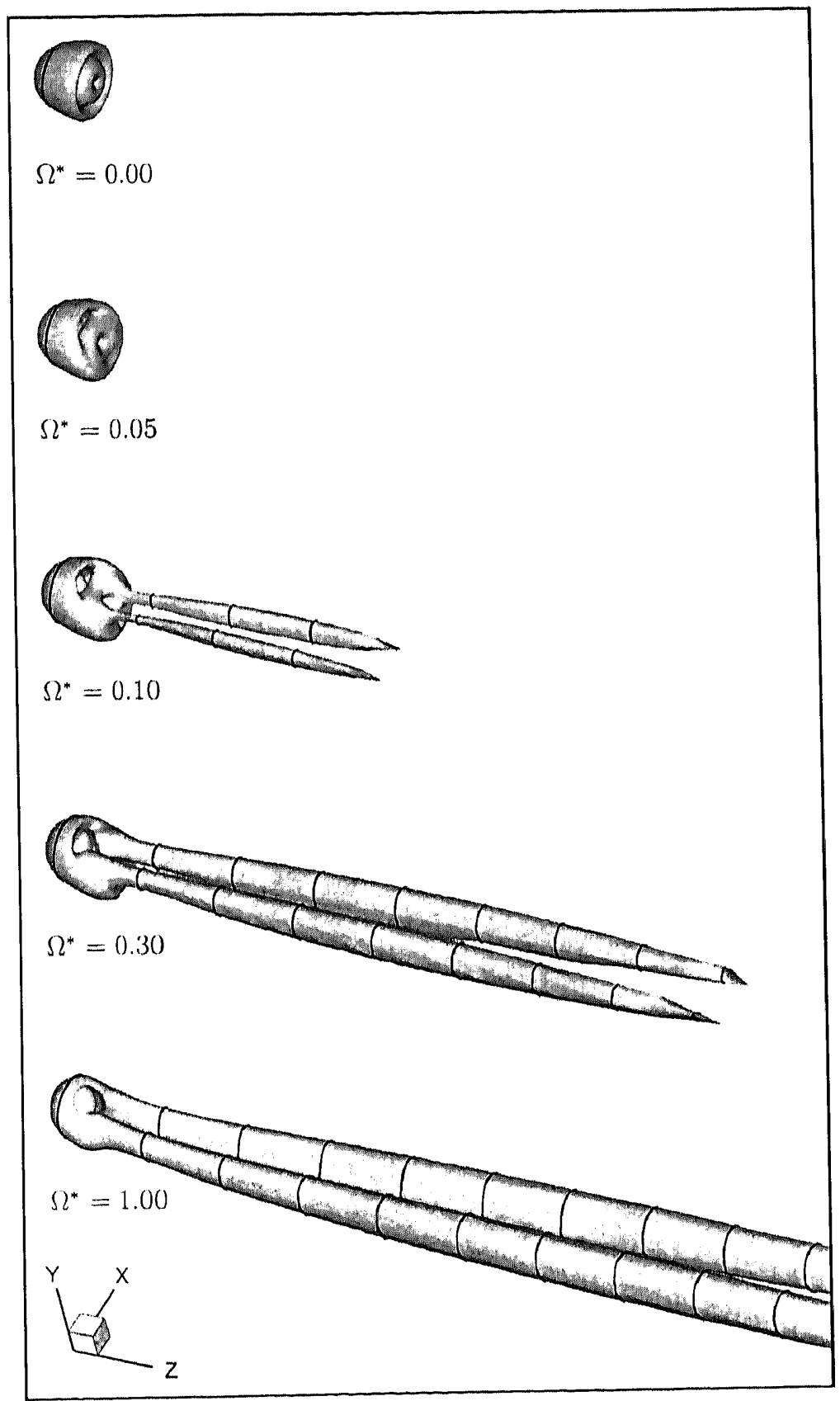

Figure 5.1: Vortical structures for $R e=100$. 


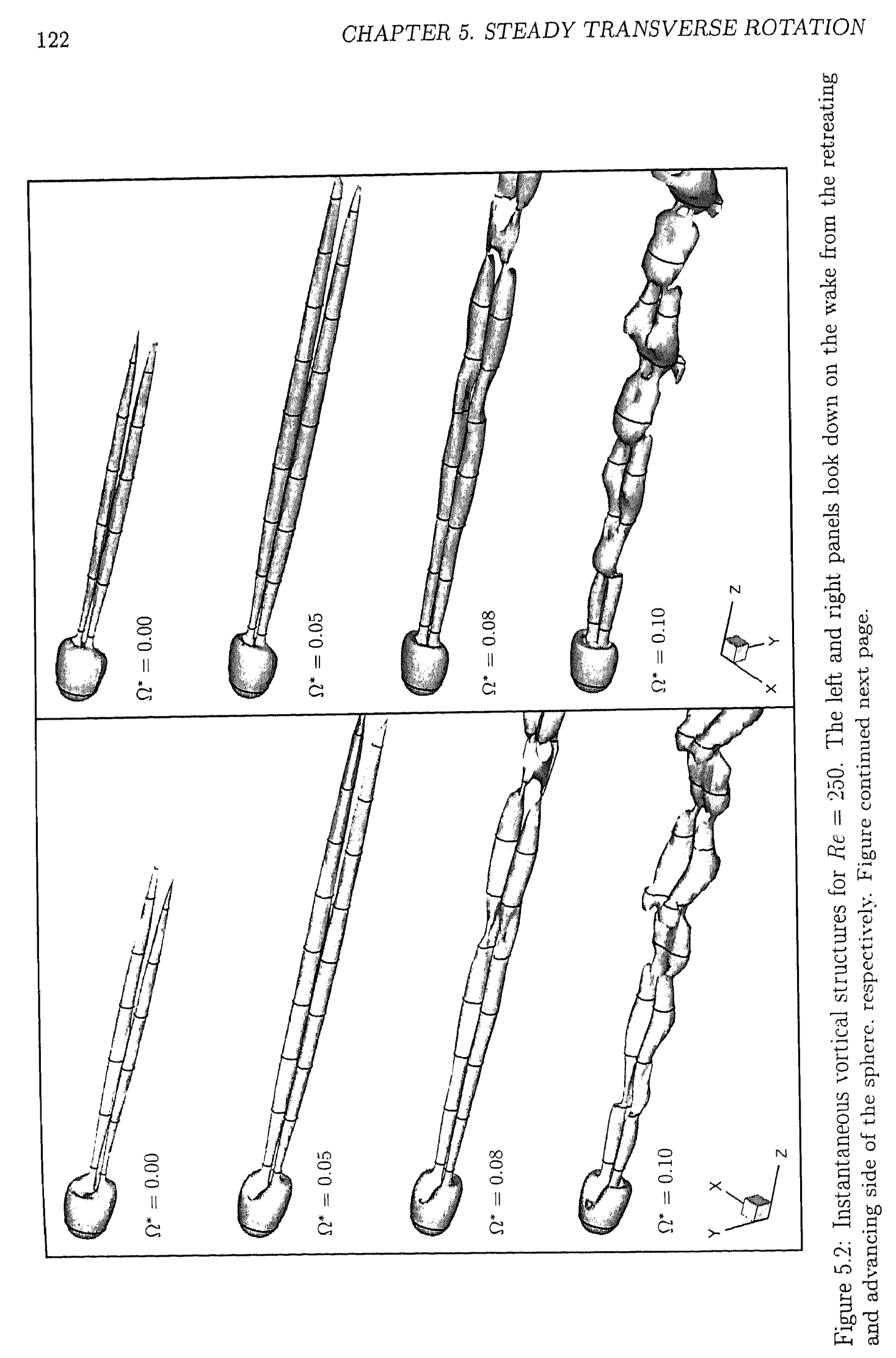




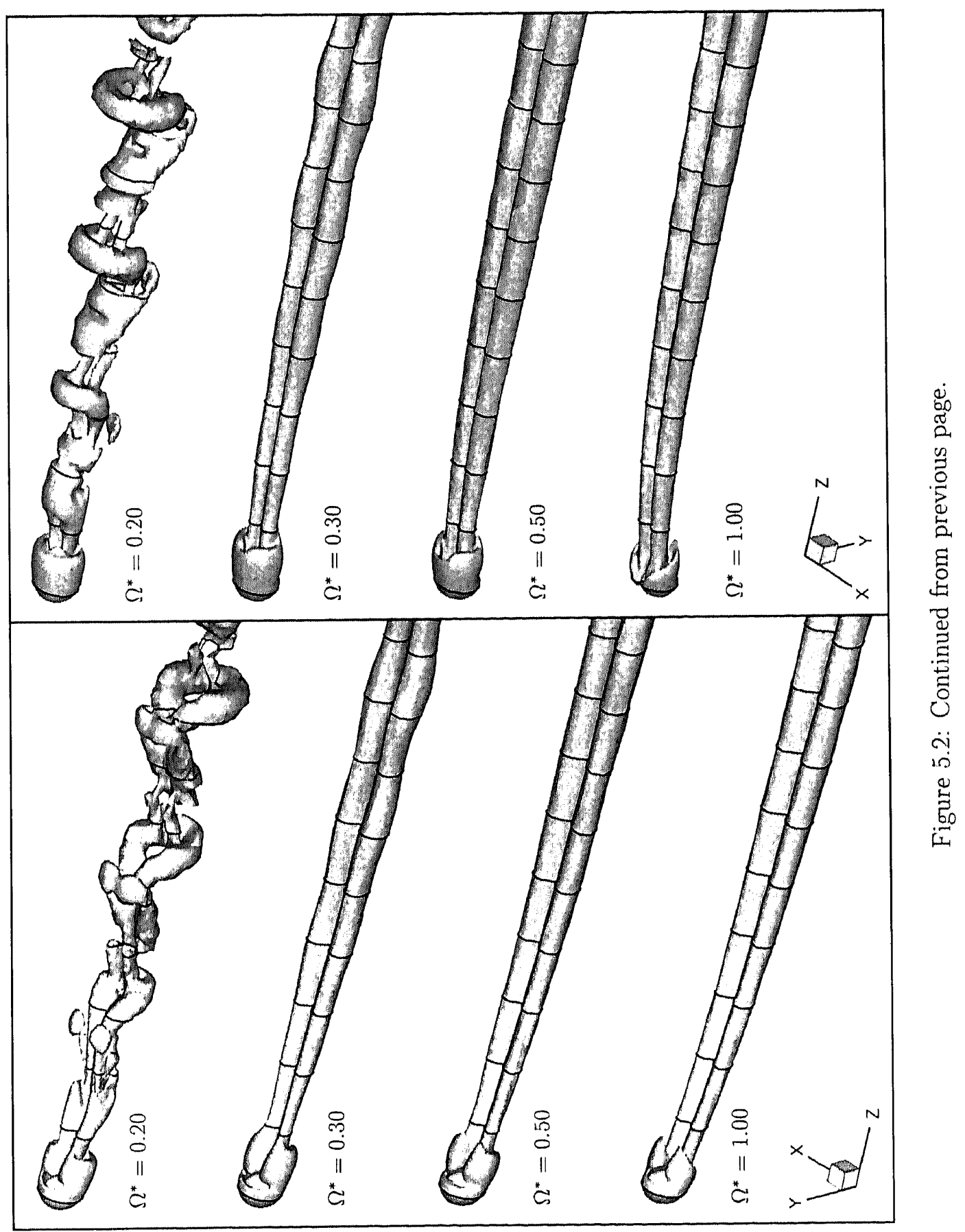


For $\Omega^{*}=0.10$ the streamwise tails which characterize the wake at lower $\Omega^{*}$ undergo a cycle of growth and release into the near-wake. As these structures are convected downstream they become connected near their downstrcam end by a bridge, such that a hairpin-type structure is formed. The formation of these hairpins is best illustrated starting at $t_{c}^{*}=3 T / 4$, where the bridge (A) is in its initial stages of formation. This bridge intersects the streamwise threads slightly upstream of their downstrcam tip, thus leaving streamwisc protuberances downstrcam of the bridge (C). As the structures move downstream, they are also convected in the negative $y$-dircetion due to their mutual induction. This effect is strongest near the middle of the hairpin structures (noar $B$ for example), where the distance between their legs is smallest. The higher mutual induction in this region leads to a kink in the vortical structure. Upstream of the kink, the structure becomes progressively tilted away from the streamwise direction until, in the far-wake, the legs reconnect to form a secondary upstream hairpin structure (D). The downstream section remains relatively parallel to the streamwise direction.

For $\Omega^{*}=0.20$, the wake shows some similarity to the wake of a stationary sphere at Reynolds numbers above the Hope bifurcation (Re > 270)). Likc-sign hairpin structures (A) are shed from the sphere and as they are convected downstrenn they become tilted away from the streamwise direction and assume a $\Omega$-shape. As in the case for $\Omega 2^{*}=0.10$, streamwise protuberances (B) extend downstream of the hairpin structures shed from the sphere, but are more pronounced at, this higher rotation rate. In the near-wake there is also the appearance of hairpin structures (C) of opposite oricutation and sign to these shed from the sphere. The heads of the induced structure form around the legs of the hairpins shed from the sphere. In the absence of other clfects, it, would appear that these induced vortices are responsible for convecting the legs of the shed vortices in the positive $y$-direction and bringing them together, in accordanes with the Biot Savart law. This leads to a reconnection of the shed hairpins and the formation of vortex ring-type structures in the far-wake, such as that labelled D.

The effect of sphere rotation on the flow structure at $R e=300$ is shown in figure 


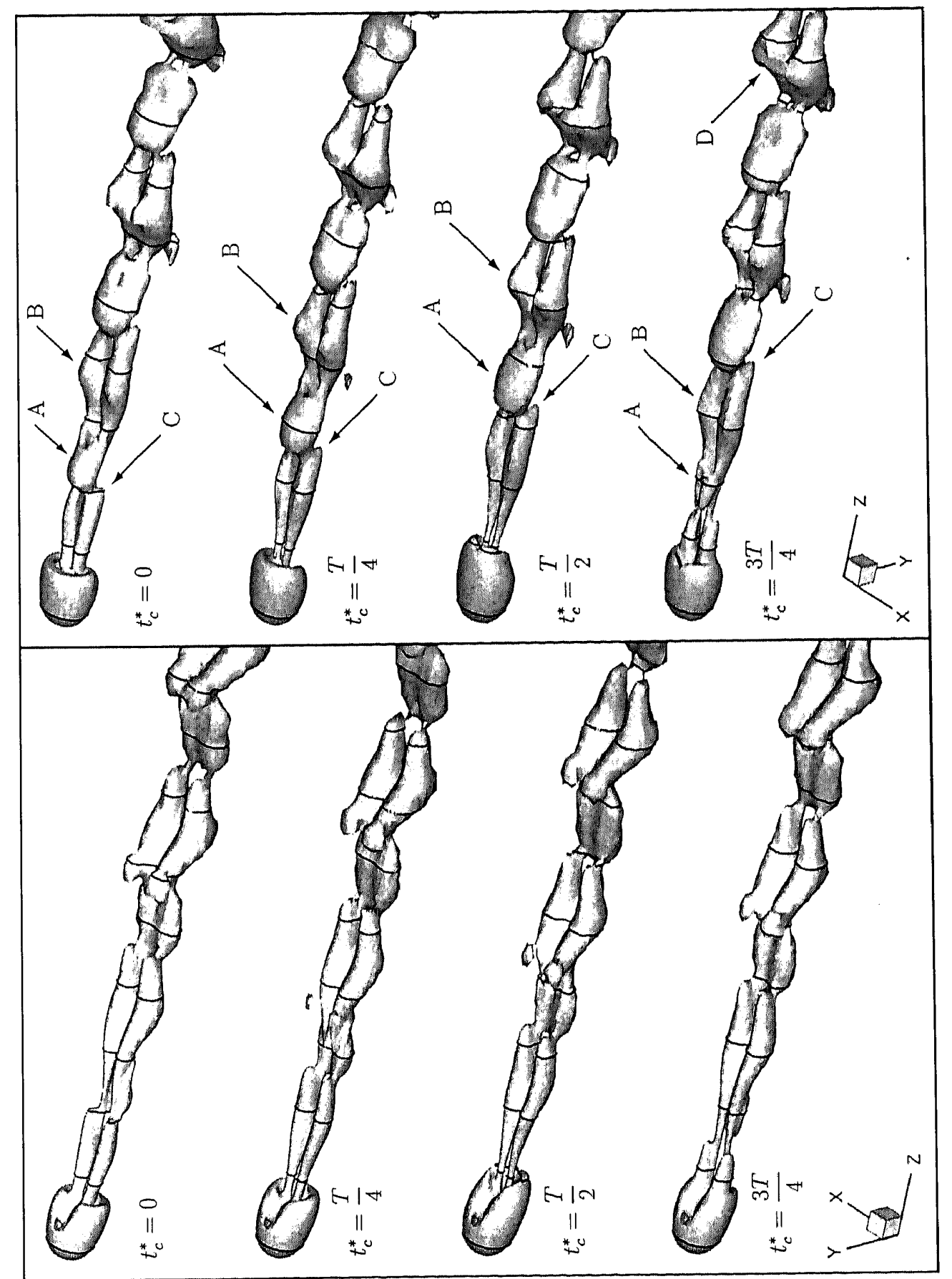

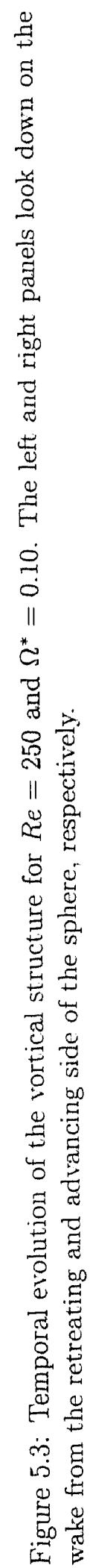




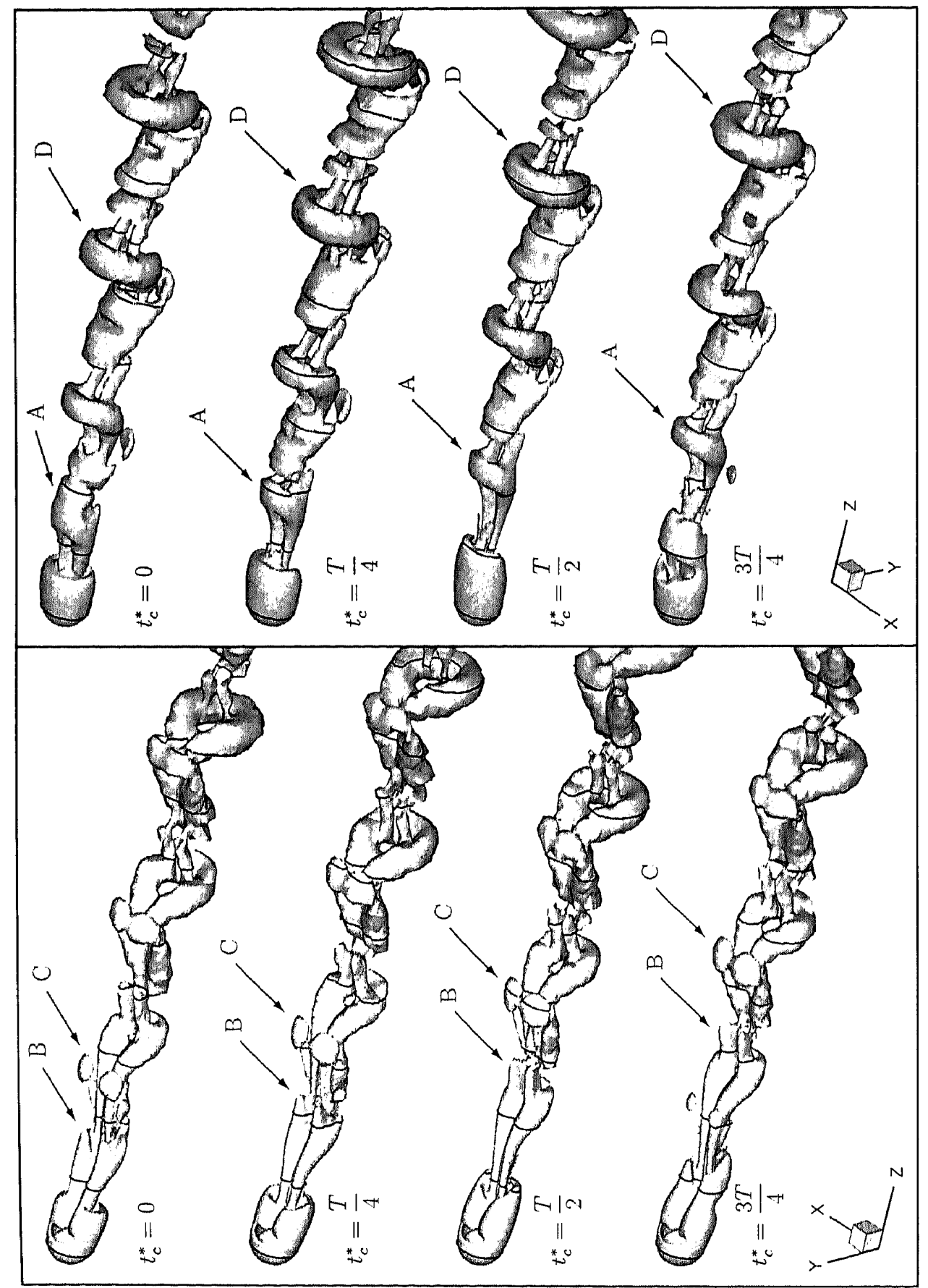

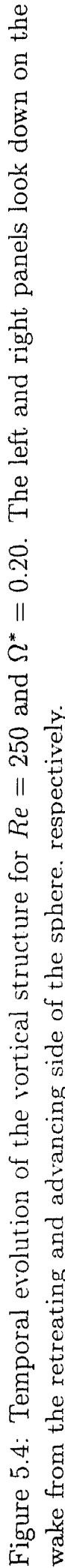


5.5. At this Reynolds number, the flow past a stationary sphere is characterized by the periodic shedding of like-signed hairpin structures (A) and the appearance of induced hairpin structures (B) of opposite sign and orientation. Johnson \& Patcl (1999) argued that these induced structures arise from an interaction of the near-wake and the outer flow. For a uniform and incompressible frecstream flow, vorticity cannot be generated away from the sphere surface, so these induced structures must somchow arise from the redistribution of vorticity generated at the surface. Over the range of $\Omega^{*}$ considered, the wake undergoes a number of interesting topological transitions. With increasing $\Omega^{*}$, the lateral expansion of the wake increases initially before decreasing again until vortex shedding is suppressed in the range $0.50<\Omega^{*}<0.60$ and the wake reverts to a stcady doublc-thread. This transition is also observed for $R e=250$, but at a slightly lower value of $\Omega^{*}$. In this case however, for $\Omega^{*}>0.60$, the wake undergoes a further transition to a second vortex shedding regime, which to the authors knowledge, has never been reported for a sphere. For a two-dimensional cylinder at Re $=200$, Mittal \& Kumar (2003) found vortex shedding to be suppressed for $\Omega \Omega^{*} \geq 1.91$ and to resume for $\Omega^{*} \approx 4.4$ to 4.8 . It is not clear why vortex shedding is suppressed and resumes at a significantly higher rotation rate for a two-dimensional cylincler.

In the second vortex shedding regime, the wake shows a distinctly different topology and the process by which discrete vortical structures are formed and released downstream appears to be different, suggesting that a different mechanism is driving the unsteadiness. For $\Omega^{*}=0.80$, the vortex shedding does not originate in the spliere nearwake, as is the case at lower $\Omega^{*}$. The double-thread structure extends some distance downstream from the sphere before waviness is observed in the threads, they then till, and comnect to form a compact series of interconnected vortex loops. Qualitatively, this wake development resembles that of the buoyant co-flowing wake studied by Perry \& Lim (1978) and Perry \& Tan (1984). Here, oblique Kolvin Helmholtz-like instabilities develop in the cylindrical vortex shect, causing it to develop folds which lead to the formation of one-sided structures that rescmble a 'daisy chain' of interlocking 
loops. For $\Omega^{*}=1.00$, the formation of the hairpin structures occurs further upstream; otherwise, the flow topology is similar to that at $\Omega^{*}=0.80$. It is interesting to note that this second transition to a vortex shedding regime docs not occur for $R e=250$ and $\Omega^{*} \leq 1.00$, despite the similaritics in the flow structure at lower $\Omega^{*}$. This raises the question of whether at lower Reynolds number, the transition to the second vortex shedding regime is triggered at a higher value of $\Omega^{*}$. To investigate this possibility, further simulations need to be conducted for $\Omega^{*}>1.00$.

With increasing $\Omega^{*}$, the distance between successive wake structures is progressively reduced, indicating an increase in the vortex shedding Strouhal number. The size of the induced vortices become progressively smaller in relation to the primary structures shed from the sphere, such that for $\Omega^{*} \geq 0.80$, there is no evidence of the induced structures.

Time sequences of the periodic wake for $R e=300$ are presented only for $\Omega^{*}=0.00$, 0.30 and 0.80 . These cases were chosen because they show distinct changes in the wake topology over the range of $\Omega^{*}$ considered. The wake evolution for $\Omega^{*}=0.00$ is shown in figure 5.6. The hairpin structure shed from the surface $(\Lambda)$ is secen to originate from the structure enveloping the sphere. This structure becomes elongated in the strcamwisc direction and develops folds (most clearly cvident for $l_{*}^{*}=3 T / 4$ ), before breaking away from the near-wake to form a well defined hairpin structure. The legs of this hairpin remain connected to the near-wake until a new structure (C) is formed around its legs. This new structure grows to form a hairpin of opposite sign ancl oriontation to that shed from the sphere. As the hairpins shed from the sphere are convected downstream, a kink is formed mid-way along their streamwise extont, (I3), similar to that, described for $R e=250$ and $\Omega^{*}=0.10$. This kink leads to the formation of a secondary hairpin through a reconnection, which then grows and becomes tilted away from the streamwise direction as it travels downstream, while the primary hairpin maintains its strcamwisc orientation. Kim \& Choi (2002) observed a very similar wake evolution and noted that the formation of a second upstream hairpin is not obscrved in 

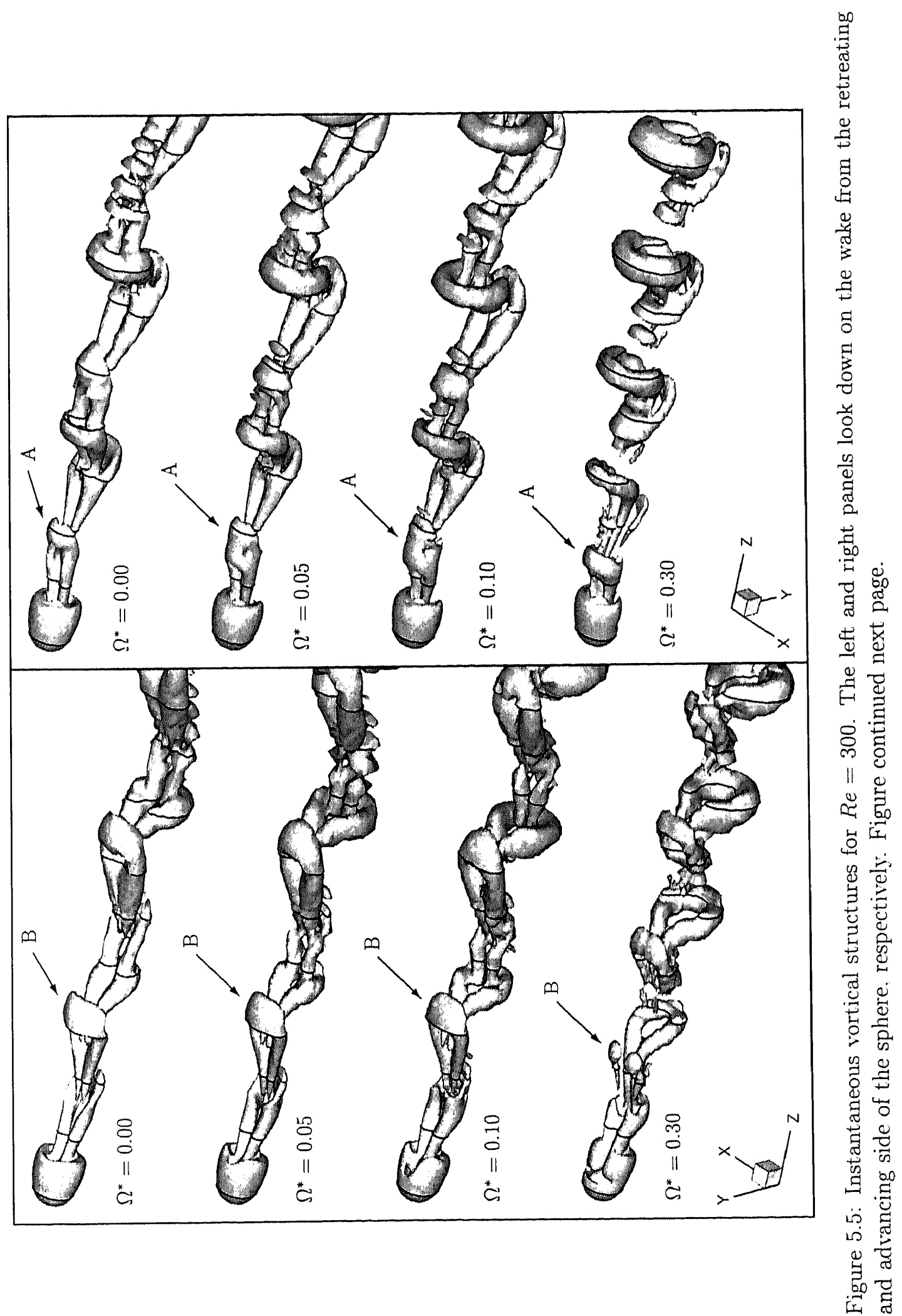


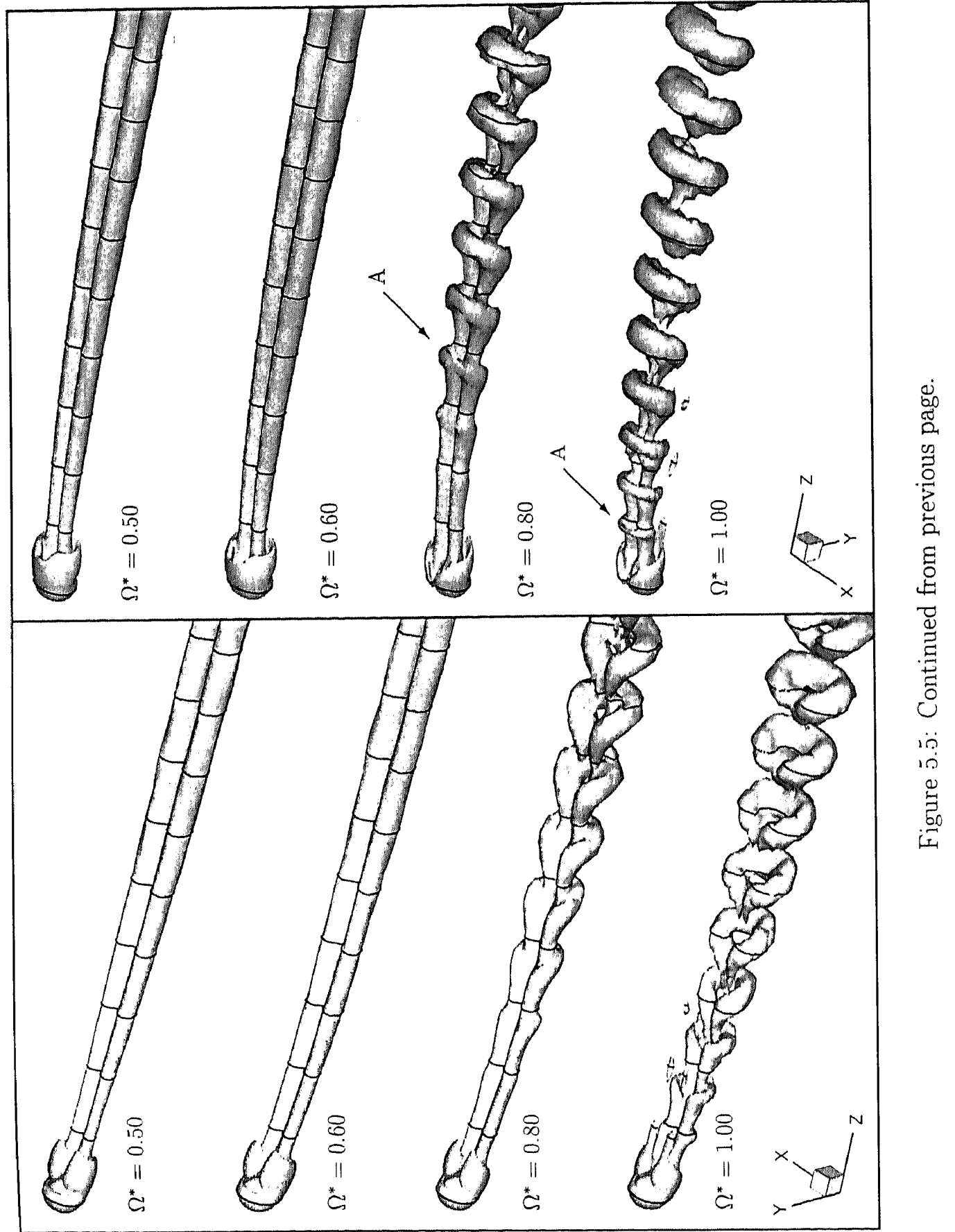


the numerical study of Johnson \& Patel (1999). They also noted that a similar hairpin evolution has been observed in numerically simulated turbulent wall layers. Zhou et al. (1996), for example, simulated the evolution of a single hairpin vortex-like structure in a low Reynolds number turbulent channel flow. Provided that the initial strength of the primary vortical structure was sufficiently high, Zhou et al. observed the generation of a secondary hairpin vortex through a similar process to that described here for the sphere wake flow.

The wake evolution for $\Omega^{*}=0.30$ is shown in figure 5.7. In this case, as the head of a hairpin structure (A) separates from the structure enveloping the sphere, it immediately assumes a curled-up $\Omega$-shape. The legs of this structure are closest at their upstream end, thus eliminating the conditions that lead to the formation of the secondary upstream hairpins that are formed at lower $\Omega^{*}$. With reforence to figure 5.5, it is evident that as the rotation rate is increased in the range $0.00 \leq \Omega^{*}<0.30$, the secondary hairpins (B) progressively decrease in size, while the primary hairpins (A) become more prominent.

The wake evolution for $\Omega^{*}=0.80$ is shown in figure 5.8. Although not obvious from the figure, the streamwise tails show a small, symmetric periodic displacement that is most pronounced in the $y$-direction. This waviness grows spatially until the streanwise structures reconnect to form a scries of like-signed hairpins. As the wake structures are convected downstream, the hairpins become tilted away from the strcamwise direction and assume a more distinct $\Omega$-shape. The mechanism responsible for this transition to vortex shedding is not cortain, although a Kelvin Helmholtz instability of the shear layer separating from the advancing side of the sphere appears to be the likely cause. On the arlvancing side of the sphere, the surface moves in the opposite direction to the frecstream velocity. An increasing rotation rate leads to a higher relative velocily and the intensification of the shear layer separating from this side of the sphere (this is 
shown more clearly in $\S 5.3$, where the vorticity ficld is presented). $\uparrow$ An increase in the rotation rate also leads to the intensification of the streamwise vortical tails, thus an instability of the low pressure cores cannot be ruled out as the trigger to unsteadiness. It must be cmphasized that this system is more involved than that of a free shear layer or a contra-rotating vortex pair, with which analogies have been drawn here. The shear layer on the advancing side forms a three-dimensional shect which foeds into the contra-rotating vortex pair. Thus, the mechanism responsible for the transition to vortex shedding may be different to that found for a parallel free shear layer or a parallel contra-rotating vortex pair.

Over the parameter range tested, the wake maintains a planar symmetry normal to the sphere rotation axis. To verify that this symmetry plane is physical and not a result of an inadequate symmetry breaking perturbation, a number of initial perturbations, different to that described in $\$ 2.6$ have becn considered. Figure 5.9 illustrates a symmetry broaking test applied to the casc of $R e=300$ and $\Omega^{*}=1.00$. The highest Reynolds number simulated is presented here because it is expected to be most, sensitive to symmetry breaking perturbations. The time secpuence shown begins with the statistically steady flow for a transversely rotating sphere at, $l^{*}=3(0) .0$, followed by an impulsive change to strcamwise rotation for $300.0<1^{*}<332.5$. This gross perturbation breaks the planar symmetry and structures spiral about the streanwise axis as they are convected downstream, in a similar fashion to the wake structures computed by Kim \& Choi (2002) for a sphere in stcaly streamwise rotation. (Onec rotation in the transverse sense is impulsively restored at $t^{*}=332.5$, the wake symmetry plane is quickly re-established without hysteresis. Quantitatively, the symmetry of the wake can be ascertained by monitoring the zeroth azimuthal moske of $u_{\phi}$. The zeroth azimuthal mode, $\widehat{u_{\phi}}(k=0)$, can be regarded as the average azimuthal velocity, and should be zero if a plane of symmetry exists. Figure 5.10 presents the time-history of

$\dagger$ A shear layer is not identified using the $\lambda_{2}$ definition, which by its very clefinition cloes not isolate strain dominated regions. 
$\widehat{u_{\phi}}(k=0)$ at two stations in the wake, and it illustrates that once transverse rotation is restored the symmetry plane is reestablished. This test indicates that the perturbation described in $\$ 2.6$ is adequate and the flow symmetry plane is physical.

Figure $5.11(a)$ and $5.11(b)$ present the wake topology derived from the timeaveraged velocity field at $R e=250$ and $R e=300$. Only those cases for which the temporal evolution of the wake has becn described are shown. For Re $=250$ and $\Omega^{*}=0.10$, the time-avcraged wake comprises a double-thread structure, while for $R e=250$ and $\Omega^{*}=0.20$, a pair of strcamwisc protuberances $(\mathrm{A})$ are also cvident. These comprise streamwise vorticity of like-sign to the primary double-threads. For $R e=300$ and $\Omega^{*}=0.00$, the time-averaged wake comprises a primary pair of streamwise vortical threads (B) and a distinct secondary pair (C). The secondary threads comprise streamwise vorticity of opposite sign to the primary threads and are the timeaveraged signature of the induced hairpins that are seen in the instantaneous flowfield. For $R e=300$ and $\Omega^{*}=0.30$, the primary pair of streamwise structures are thicker and secondary protuberances $(D)$, similar to those found for $R e=250$ and $\Omega^{*}=0.20$, are evident. The protuberances appear with the lateral expansion of the instantaneous wake, where the hairpin structures shed from the sphere become tilted away from the streamwisc axis and assume a $\Omega$-shape. For $\Omega^{*}=0.80$, the protuberances are absent and the time-averaged field shows only the primary set of vortical threads.

Despite distinct topological differences in the instantaneous flow, the time-averaged walke is essentially characterized by a pair of counter-rotaling vortical threads. As hairpin structures are convected downstream, their legs maintain a quasi-streamwise orientation. The resultant streamwise spiraling motion is reinforced by cach consecutive structure, such that a pair of trailing vortices is always evident, in the time-averaged ficld. The bridge or head of the hairpin structures comprise spiraling motion that is approximately normal to the streamwise direction (along the $x$-axis). The velocity with which the structures are convected downstream is superimposed onto this spiraling motion, such that the hairpin heads do not produce spiraling motions in the 
time-averaged velocity field. In hindsight, the existence of a trailing vortex pair in the time-averaged velocity field is not surprising, since, for a threc-dimensional body that is gencrating lift, the laws of inviscid vortex motion dictate that trailing vortices must exist. $\dagger$ The presence of a pair of counter-rotating vortical threads in the timc-averaged wake of a transversely rotating sphere has been previously reported by Maccoll (1928) for a higher Reynolds number $\left(R e>10^{4}\right)$. Using a threc-component pressure gauge, Maccoll measured the time-averaged flow direction and speed over a number of cross-stream planes downstream of a sphere mounted in a wind-tunnol. Calculated streamwise vorticity contours indicated the presence of a pair of vortex cores that were deflected towards the advancing side of the sphere.

$\dagger$ The Kutla -Joukowski law relates the lift generated by a body, $L$, to the circulation, $K$, through $L=\rho U_{\infty} K$. The net circulation, $K$, can be considered to comprise a bumdle of vortex filaments running along the span of the body to form a 'lifting line'. From Helmhelly second theorem, lhese filaments cannot terminate in the interior of the flud. Since the vortex filament.s move with the fluid, a simple and consistent model is that, where the vortex filaments end along the borly spun, hory turn and trail the body. These ideas form the basis of Prandtl's lifting line theory, where the lift producing body is modelled as a collection of 'horseshoe' vortices. Each vortex filament. consists of an segment along the lifting line and two semi-infinite filaments trailing behind the body. For a thorough discussion of this topic the reader is referred to the text by Milne-Thomson (1966). 

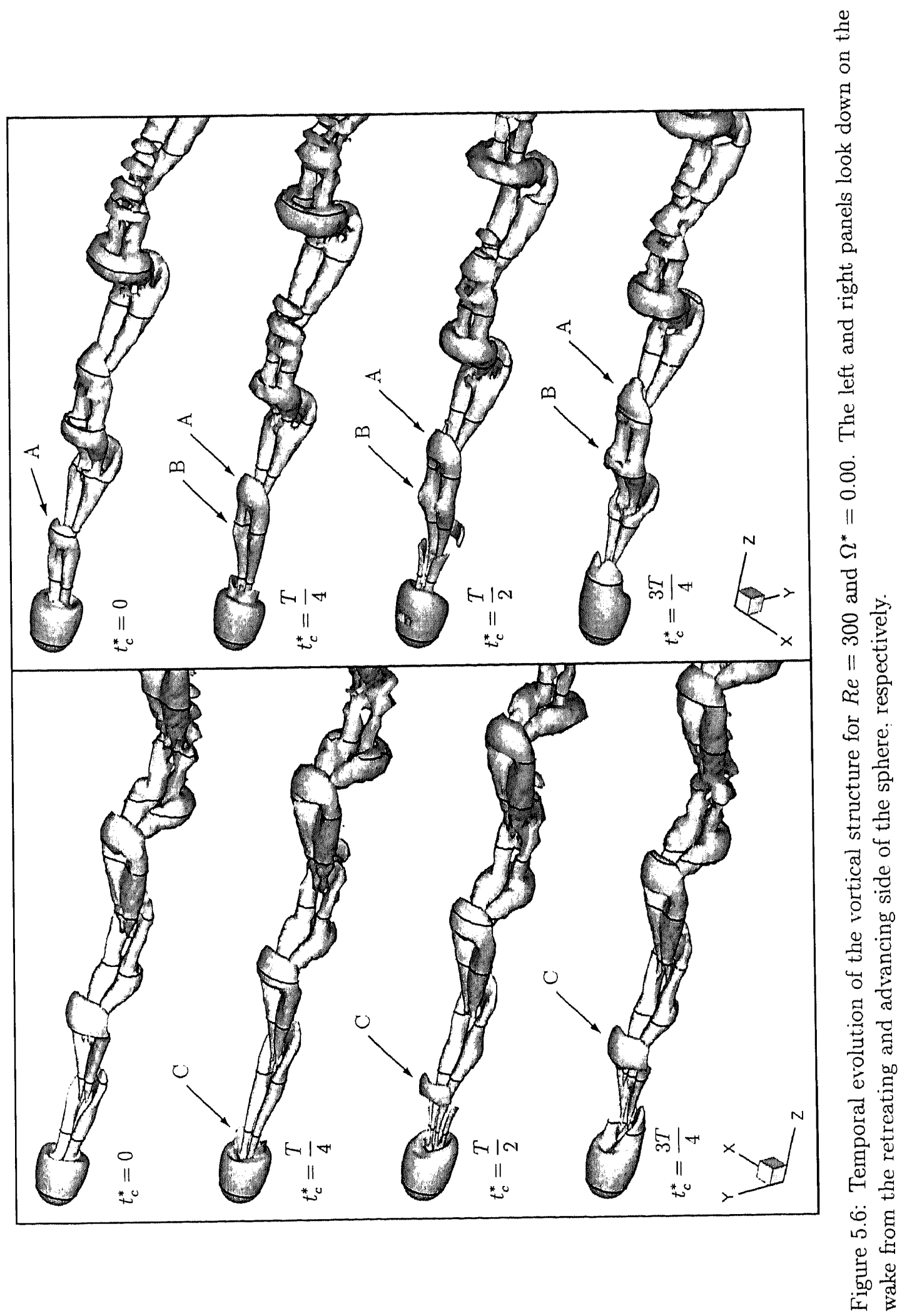

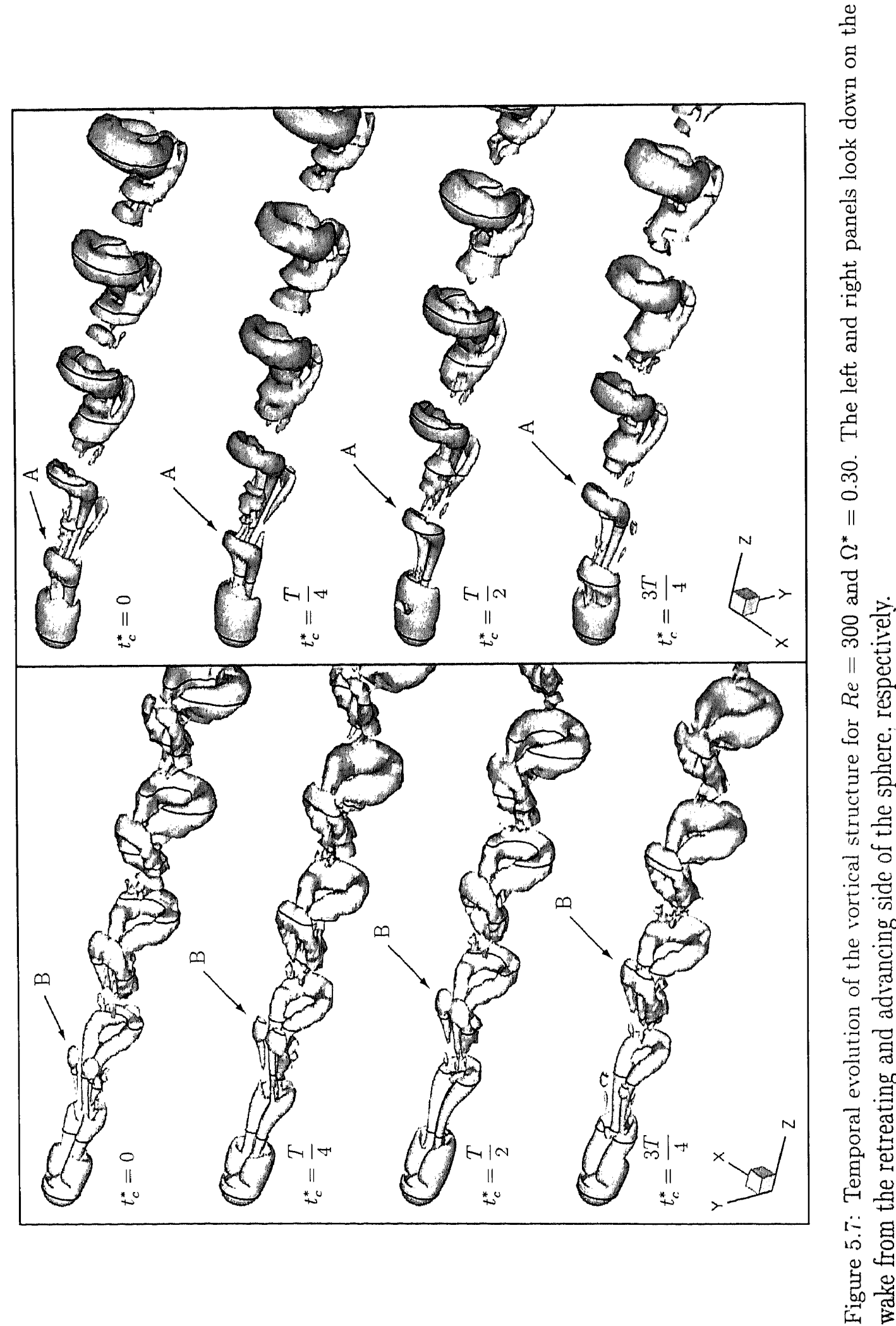

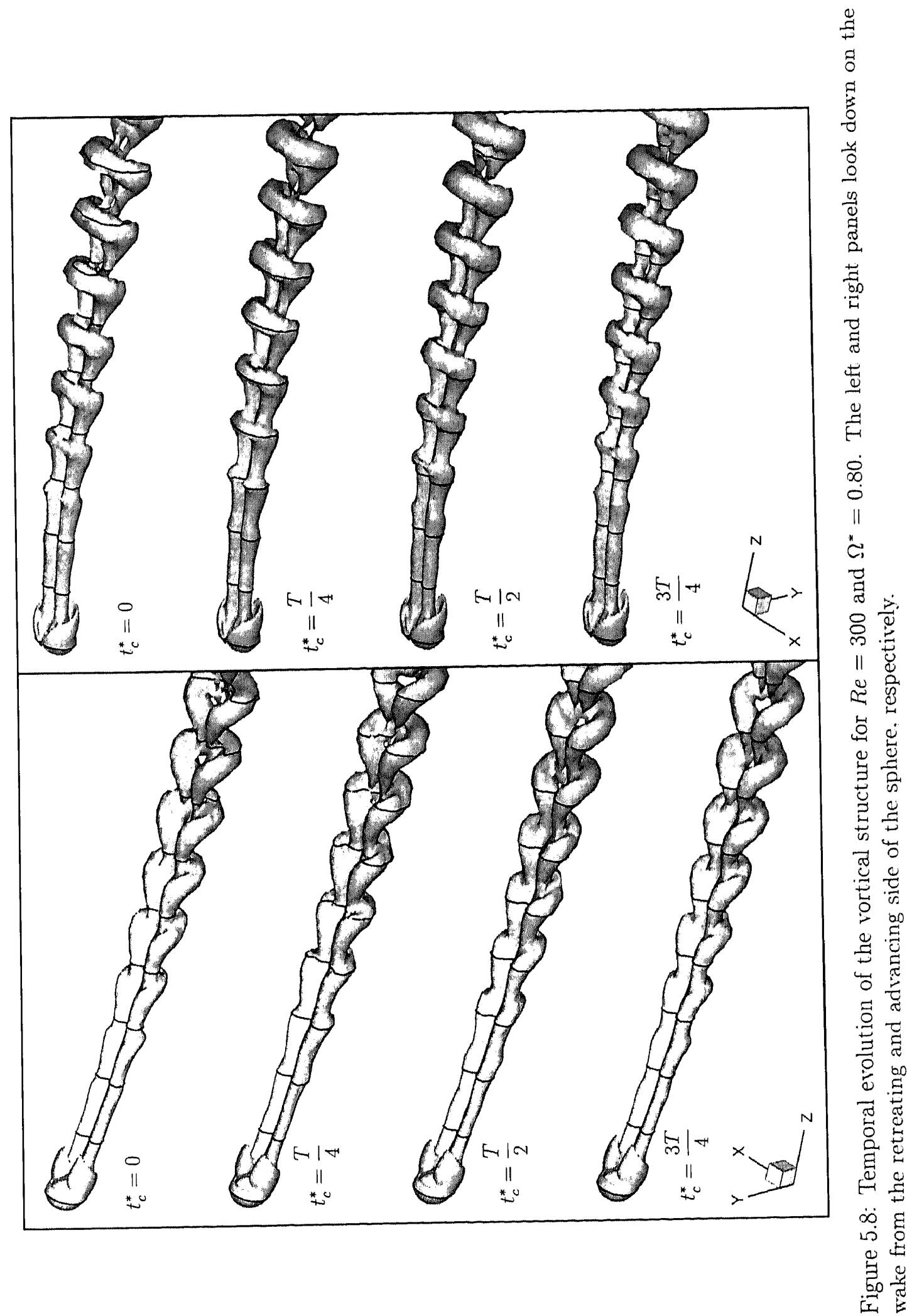


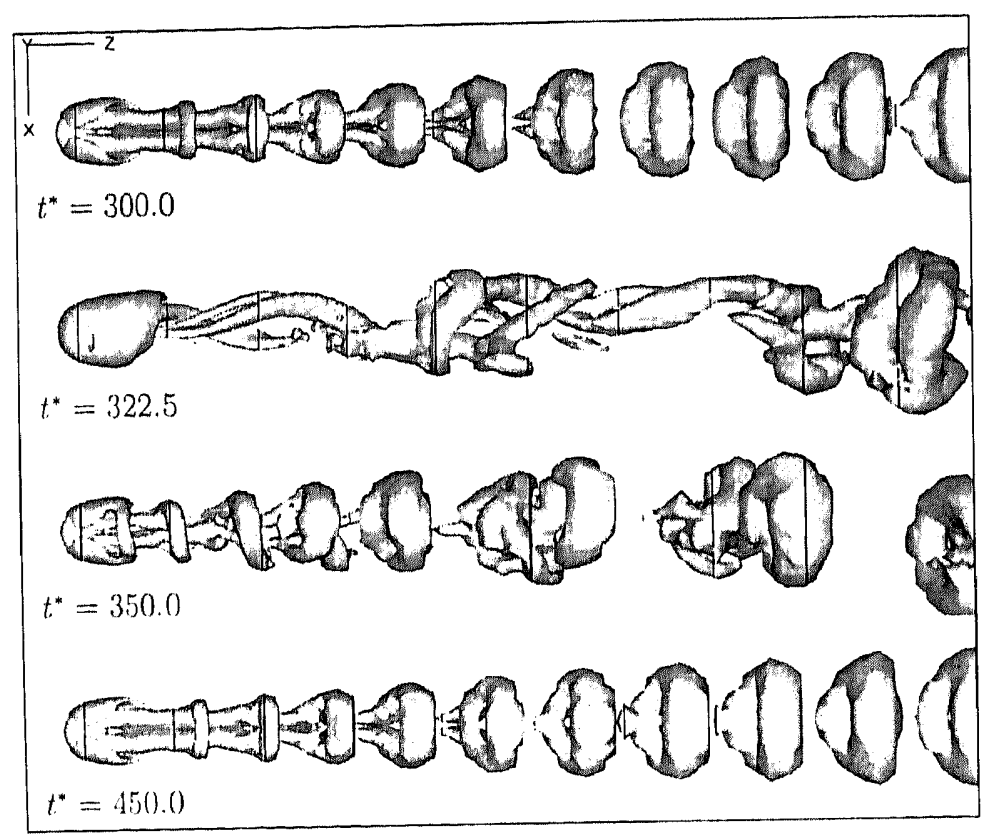

Figure 5.9: Elfect of symmetry broaking perturbation lor $R_{0}-300$ and $\Omega^{*}=1.00$. The time seguene begins with the statistically stoady flow for transversely rotating spluere at $l^{*}=300.0$. This is followed by stroamwise rotation for $3010.0<1^{*} \leq 322.5$ and transwerse rotation for $l^{*}>322.5$. Vortical structures are iclentified by plotting iso-surfaces of $\lambda_{2}=-8 \times 10^{4}$. 


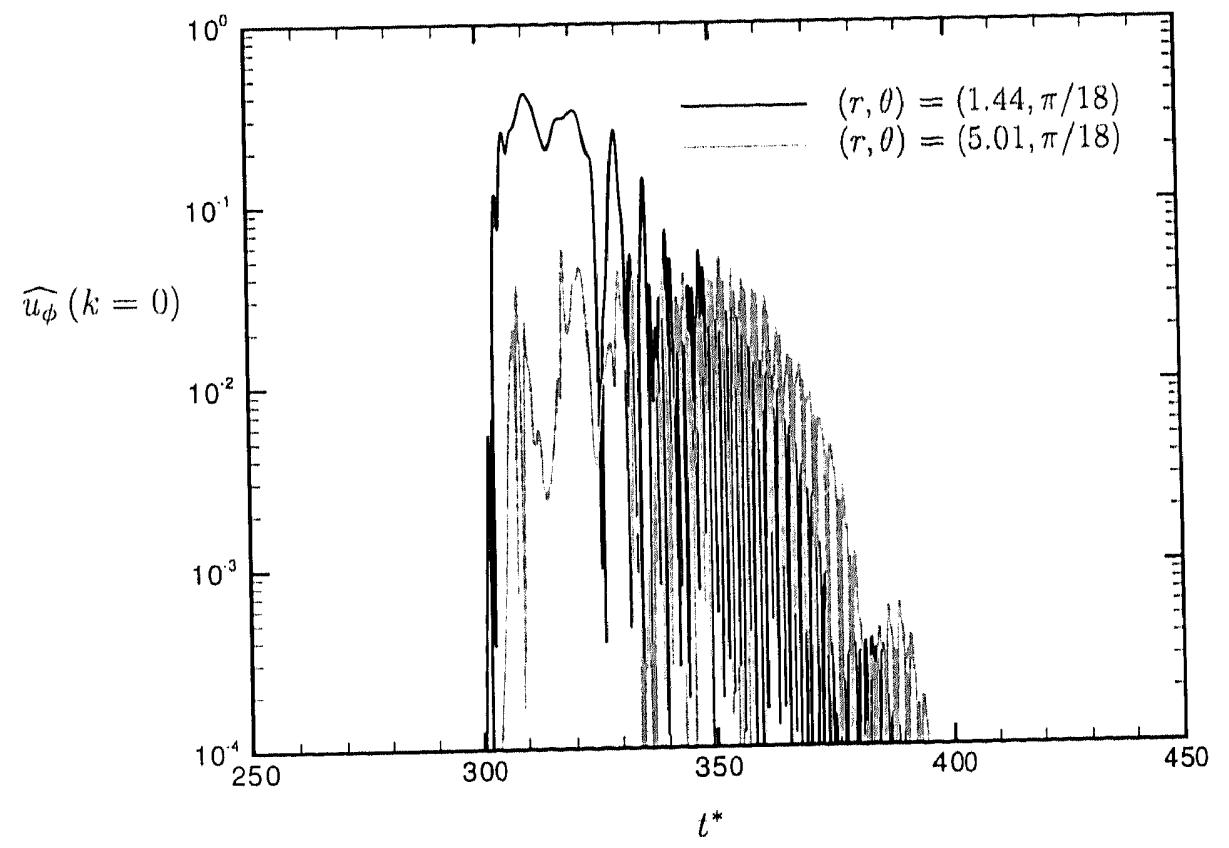

Figure 5.10: Time-history of azimuthal mode $k=0$ of $u_{\phi}$ at $(r, \theta)=(1.51, \pi / 18)$ and $(0.10, \pi / 18)$. 


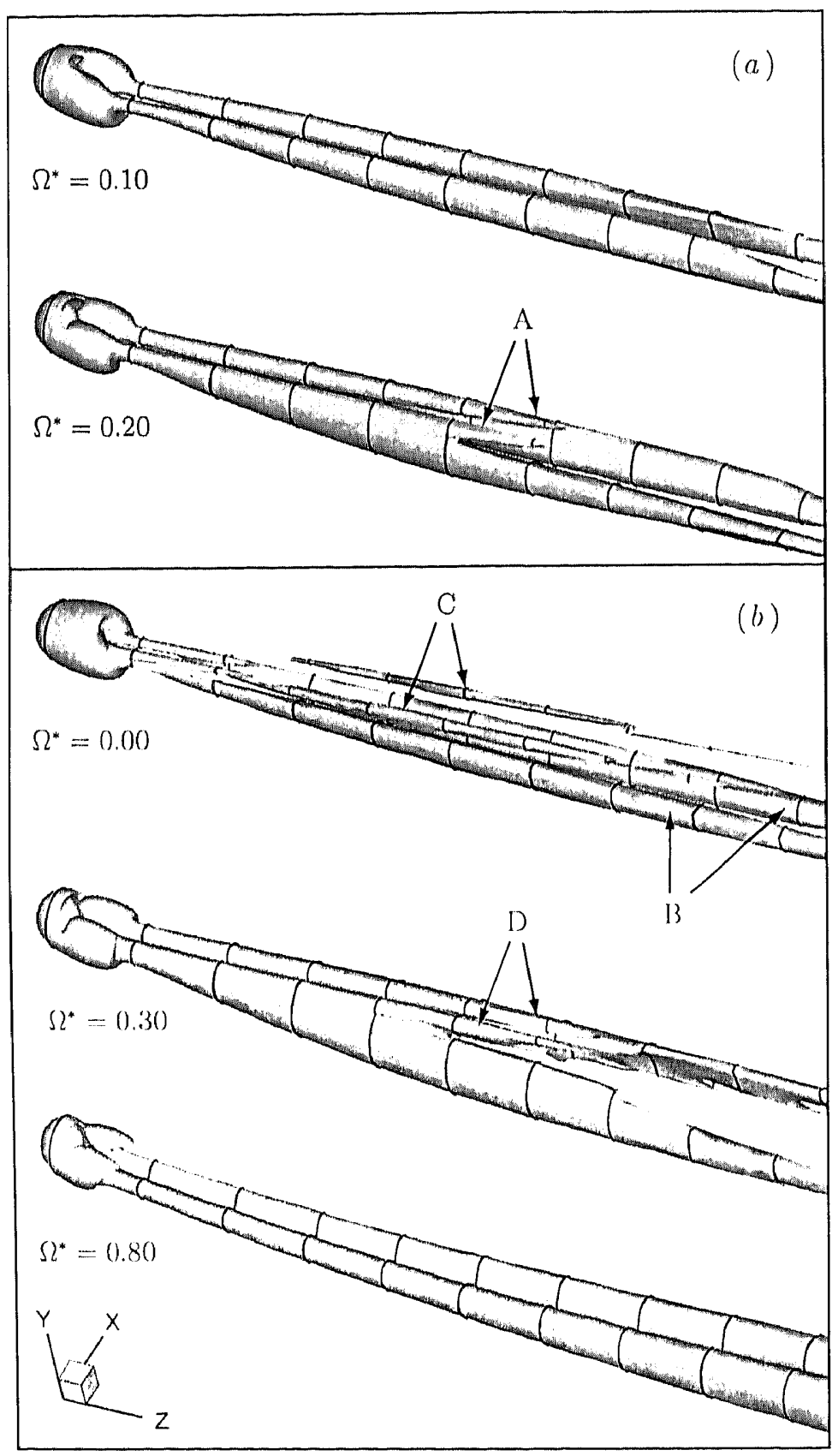

Figure 5.11: Vortical structure derived from the timc-averaged velocity field: (a) $R e=250 ;(b) R e=300$. 


\subsection{Near-wake streamline pattern}

To gain further insight into the physical processes that shape the wake, this section examines the near-wake streamline topology. Figures 5.12 and 5.13 show the nearwake streamline pattern on the $(x, z)$ - and $(y, z)$-planes, respectively, for Re $=100$ and a number of representative rotation rates. For $R \cdot c=100$ the flowficld remains steady and the patterns also correspond to fluid pathlines. For a stationary sphere, the flowficld is axisymmetric and the streamline pattorn in the $(x, z)$ - and $(y, z)$-planes is identical. The wake streamlines form concentric closed loops, or limit cycles, and a separatrix stream surface delineates fluid within the toroidal wake from the outer flow. The introduction of sphere rotation breaks the flow axisymmetry and the loroidal wake becomes tilted, however, the flow does maintain a plane of symmetry normal to the rotalion axis. Flow symmetry about the $(y, z)$-plane implies that there are no outof-plane velocity components and therefore figure 5.13 presents true streamlines. In the $(x, z)$-plane, out of plane velocity components exist and therefore figure 5.12 shows streanlines obtained by integrating the velocity ficld resolved onto the plane. These are not true streamlines and only when interpreted with care, ean they provide further insight, into the nature of the near-wake. It is for this reason that for $R e=250$ and $R e=300$, the instantancous streamline patterns on the $(x, z)$-plane are relegated to Appendix $B$.

For $\Omega \Omega^{*}=0.05$, fluid passing over the retreating side of the sphere is entrained inte the wake and is ejected from the advancing side of the sphere. For convenience, the foci on the retreating and advancing sides will be referred to as the upper and lower foci, respectively; however, it is emphasized that these foci are a two-dimensional slice of a single threc-dimensional toroidal structure. The threc-dimensionality of the flow is evident from the net divergence on the symmetry plane. The upper foeus is topologically stable and relcases fluid normal to the plane, while the lower focus is unstable and draws in fluid from either side of the symmetry plane. Figure 5.14 
shows three-dimensional streamline paths corresponding to the projected streamline patterns presented in figures 5.12 and 5.13. For clarity, only a fow paths are shown and, because the flow is symmetric about the $(y, z)$-plane, only $x \geq 0.00$ is considered. Fluid entrained into the wake from the retreating side spirals towards the stable upper focus and is ejected normal to the plane from its center. Fluid then spirals azimuthally around the toroidal wake and fecds the unstable lower focus, before spiraling radially outwarcls from its center and being relcased into the wake in two streams on either side of the symmetry plane. This wake configuration is surprisingly similar to that described by Johnson \& Patel (1999) for a stationary sphere above the regular transition.

For $\Omega^{*}=0.10$, the focal structures seen at lower rotation rates lose their spiral appearanee in a stationary referene frame. As the rotation rate is increased further, the near-wake becomes increasingly vectored in the negative $y$-direction and fluid passing over the retroating side remains attached until it mects fluid passing over the advaneing side of the sphere and is 'peeled off' to form a shear layer. This shear layer becomes increasingly vectored in the negative $y$-direction as the rotation rate is increased. Sphere rotalion also causes a thin layer of fluid to rotate with the spheres surface. Willa increasing rotation rate, viscous effects cause this layer of fluid to bereme progressively thicker. On the symmetry plane, the forward separatrix is shifted away from the surface and towards the advancing sicke of the sphere. For $\Omega^{*}=0$. 10, the toroidal wake structure is barely clisecruible, while at. the higher rotation rates it is alscont. At these higher rotation rates, fluid passing over the retreating side of the sphere is accelerated over the lee side of the sphere in a contracting band, while fluid passing over the advancing side forms a sheret that curls at, its edges. Figure 5.14 illustrales that theses motions combine to form a pair of counter-rotating vortical structures, similar to these found downstroam of a finite length wing. It is these counter-rotaling vortical structures that were identified in $\S 5.1$ as intense streamwise threads.

Figure 5.15 presents the near-wake streamline patterns on the symmetry plane for $R e=250$. For the cases that exhibit a periodic wake, the time instant shown coincides 


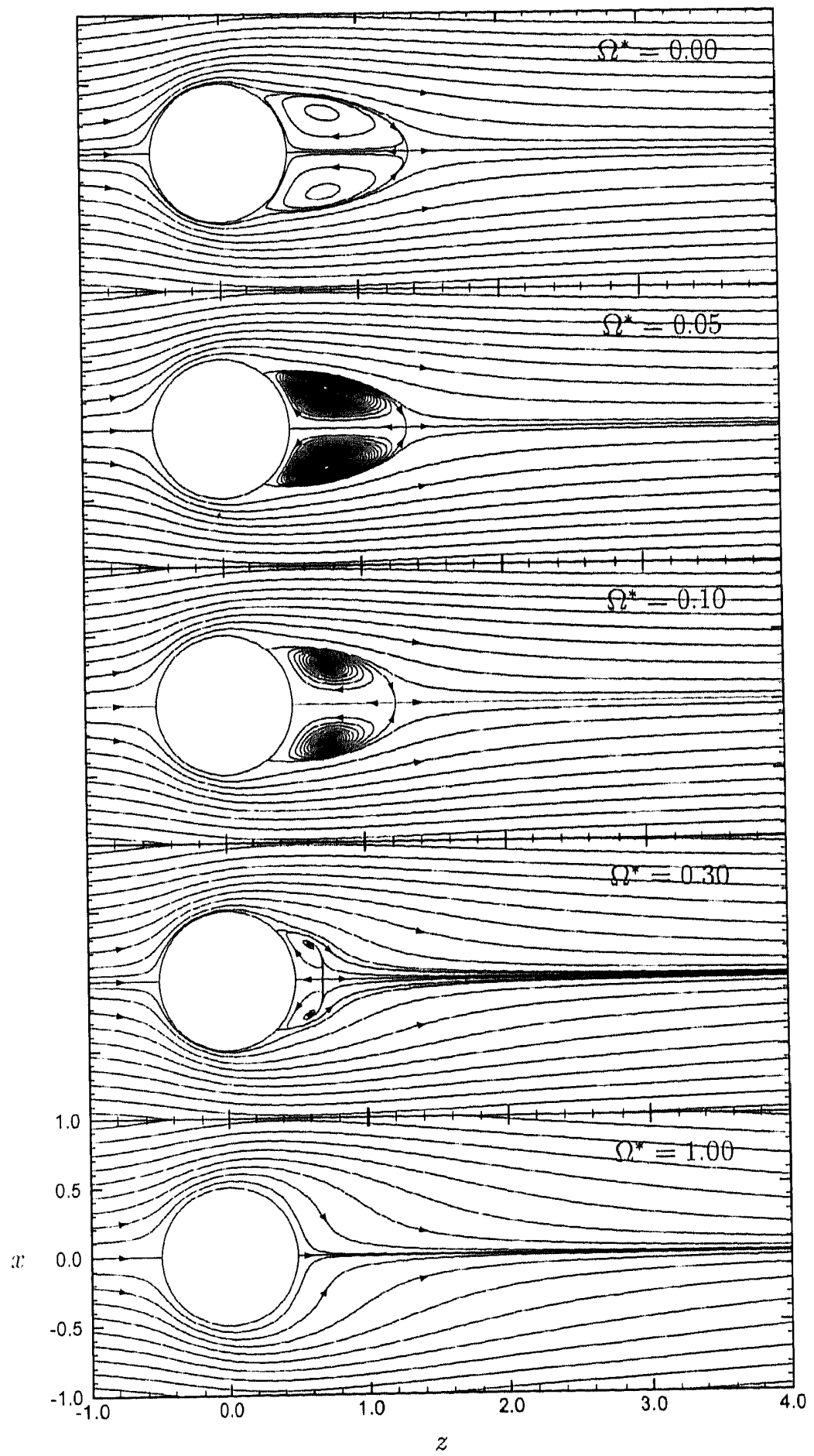

Figure 5.12: Ncar-wake streamline patterns on the $(x, z)$-planc for $R e=100$. 


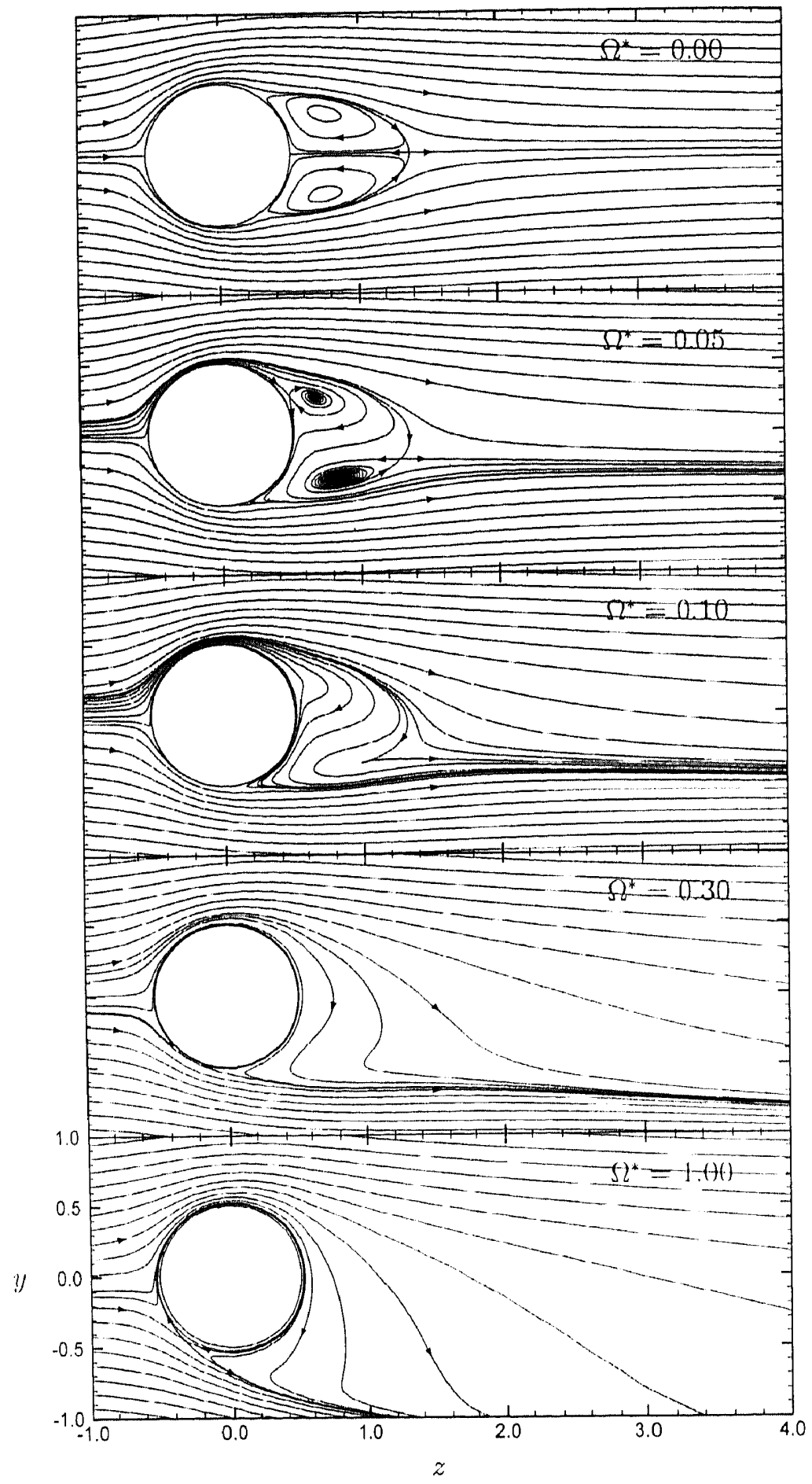

Figure 5.13: Near-wake streamline patterns on the $(y, z)$-plane for $R e=100$. 


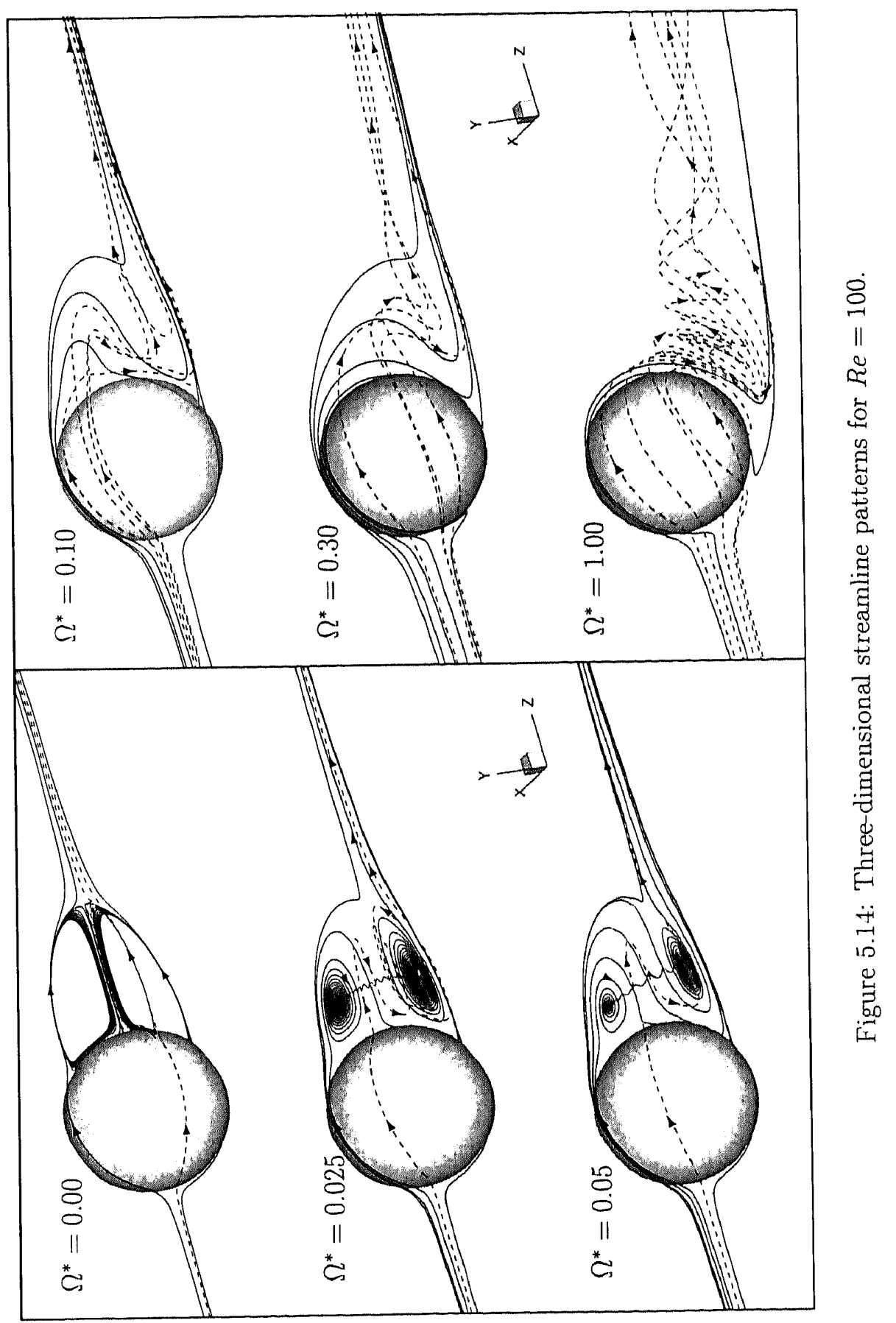


with the lateral lift force being at a local minimum, as this was judged to be most, representative of the instantancous streamline topology. These instantaneous patterns were obtained by numerically integrating the instantancous velocity field in psendo-lime. For a stcady flow, streamlines, pathlines and strcaklines are identical, making the interprctation of strcamline patterns relatively straightforward. For an unsteady flow, their relationship is more complex, and the instantancous stroamline patterns must be interpreted with care. By definition, a streamline is a solution trajectory which is tangential to the local velocity vector at each point in the flowfield. Instantancons streamlines scrve only to describe the velocity field at an instant, in time and it must always be recognized that fluid that appears to be channelled along paths prescribed by the instantancous strcamlines, may at, some later instant, follow a distinctly difforcnt path. The issue of correctly interpreting strcamline pattorns in matcady flows is discussed at length by Steiner (1984).

For a stationary sphere at $R e=250\left(\Omega^{*}=0.00\right)$, the wake exists in a stcady nomaxisymmetric regime. Although axisymmetry is lost, the wake maintains a plane of symmetry, which coincides with the $(y, z)$-plane in the present simnlations. This wake structure has been described in detail by Johnson \& Patel (1999)). Fluicl passing over the upper side of the sphere is entrained inte the wake, encirctes the nuper forms and spirals radially inwards toward the center of the lower stable focus. 'The uppore forms is unstalule and, as a consequence of mass conservation, must be fed from fluicl flowing towards the symmetry plane. This entrained flud then spirals radially ontward and is sent aremel the lower focus before being released downstream. for this case, figure 5.16 shows a pair of threc-dimensional particle pathes released on cither sicle wh the symmetry plane. A similar figure is presented by Johnson \& Patcel (1999) and serves to illustrate that the upper and lower foci, seen on the $(y, z)$-plane, are projections of the same toroidal vortex. The direction of rotation in the wake remains consistent. with that secn in the axisymmetric regime, while, in this case, the toroidal wake has become tilted and is no longer closed. Fluid which is expelled from the center of the 
lower focus, spirals azimuthally along the vortex core and feeds into the center of the upper focus. Fluid which spirals radially outward from the upper focus is sent around the lower focus before being relcased downstream, in two streams, on cither side of the symmetry planc.

Figure 5.15 shows that the introduction of sphere rotation displaces the wake foci towards the negative $y$-direction, with the size of the upper focus becoming progressively smaller, such that it is absent for $\Omega^{*} \geq 0.20$. At $\Omega^{*}=0.08$ the appearance of the upper focus changes from topologically stable to unstable. This shift in topology coincides with a transition to the vortex shedding regime. The lower focus also secs a reduction in size for $\Omega^{*} \leq 0.08$, while at $\Omega^{*}=0.10$ it undergoes a sudden growth that also marks the onset of vortex shedding. Although vortical structures are continually being shed and convected downstream, spiraling motion is only visible in the noar-wake, where the convection velocity is small. Further downstream, spiraling motions only become visible in a frame of reference translating with the local flow. For $\Omega^{*}=0.20$ the lower focus is larger still, while the upper focus is absent. It is under these conditions that the latcral expansion of the wake and the oscillatory amplitude of the force coefficient histories are found to be at their maximum.

For $\Omega^{*}=0.30$, all spiraling structures in the wake are absent and fluid passing over the lee side of the sphere appears to stay attached until it meets fluid passing over the advancing side of the sphere and is 'pecled' away from the surlace. This transition in the near-wake topology coincides with the transition to vortex shedding suppression observed in the vortical wake structure presented in the previous section. As the rolation rate is increased further, the instantancous streamlines passing over the: retreating side of the sphere become increasingly vectored in the negative $y$-direction, but otherwise the streamline pattern remains similar.

For the cases $\Omega^{*}=0.10$ and $\Omega^{*}=0.20$, time sequences of the instantaneous stroamline evolution on the $(y, z)$-plane are presented in figures 5.17 and 5.18 , respeclively. Starting at the time instant where the lift force is at a local minimum, the stream- 
line pattern is presented at every quarter period, for one period $(T)$. For $\Omega 2^{*}=0.10$, the wake comprises a pair of instantancous stable foci that are fed from fresstrenm fluid entrained from the retreating side of the sphere. This entrained fluid forms lwo stroams. A scparatrix strcamline delineates fluid that is instanlancously cntrained intos the upper focus, from that which encircles the upper focus and spiral towards the lower focus. Both the upper and lower foci are stable and expel fluid normal to the symmetry plane, which then spirals the wake before being convected clownstream. At the next. quarter cycle, the upper focus maintains its position and size, while the lower for us is displaced downstroam and appears smaller. At $t_{c}^{*}=T / 2$ the lower foches lins detached from the near-wake and is convecting downstream. In a reference frame fixed to lihe sphere it losses its spiral appearance. Fluid encircling the upper focus is drawn down to meet fluid passing over the advancing side and forms a shear layer that is subsecflucmuly convecterl clownstream. At this point, the lift coefficient attains a lesal maximum mad the drag cocflicient is in the vicinity of a local minimum. $l_{c}^{*}=3 T^{\prime} / 1$ marks the birth of a new lower focus that sits closer to the sphere surface than a mature strueture. Overall, the shedeling eycle is one of 'build-up and release' of the lower formes.

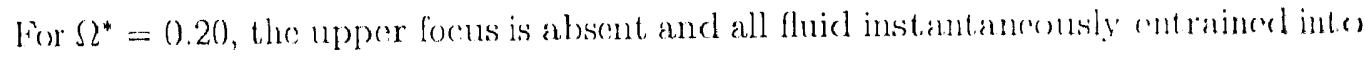
the wake is clrawn down towards the advancing side of the sphere in a strean enentracting towards the flow symmetry plane. On mecting fluid passing over the actrancing sicke

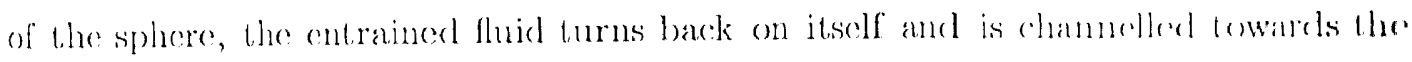
lower stable: focus. This focus undergoes a cycle of build-up) and revease, similar to that desceriber for $\Omega^{*}=0.10$.

Figure 5.19 shows the near-wake streanline patterns on the $(y, z)$-plane for Re 300 and a mumber of representative rotation rates. For a stationary sphere, the instantaneous streanlines resemble those for a stationary sphere at $R \cdot-250$. 'The most noticcable difference is a larger lower focus that sits closer to the sphere. Siphere rotallion causes the upper focus to progressively reduce in size, such that for $\left.S 2^{*}-1\right) .30$ it is absent. The lower focus also sees a reduction in size and becomes displaced further 


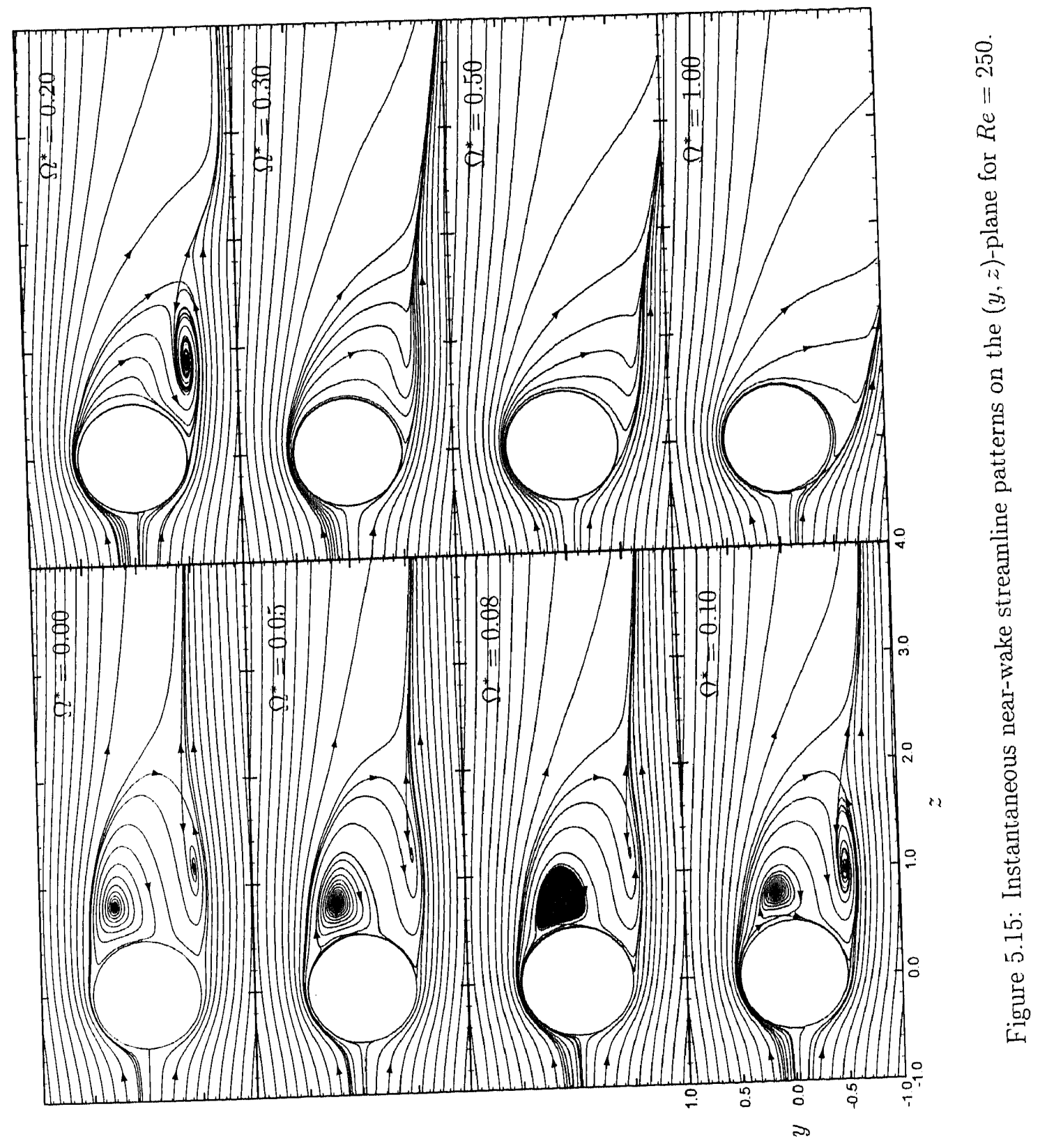




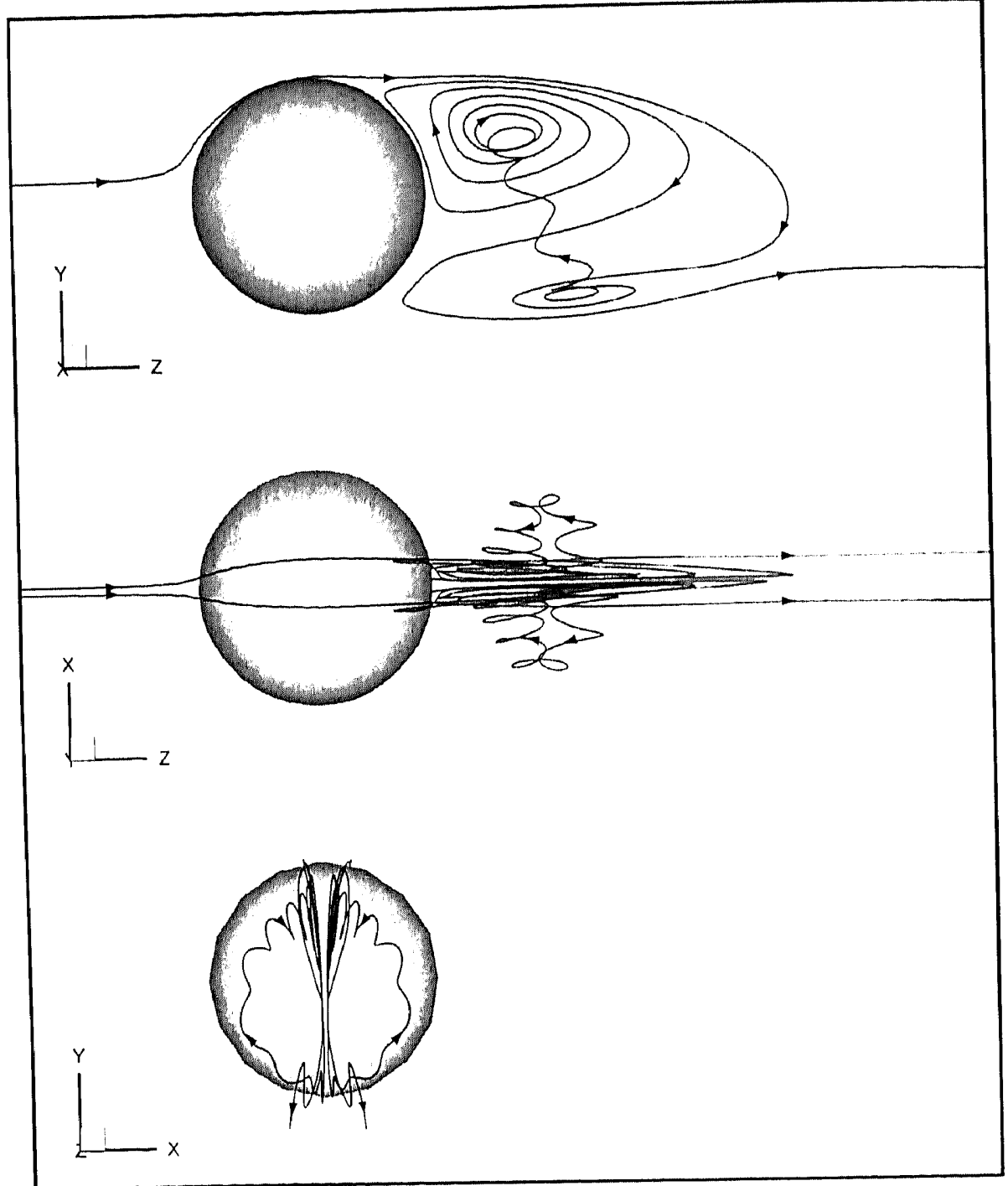

Figure 5.16: Threc-dimensional particle paths for a stationary sphere at $\operatorname{Re}:=250$. 


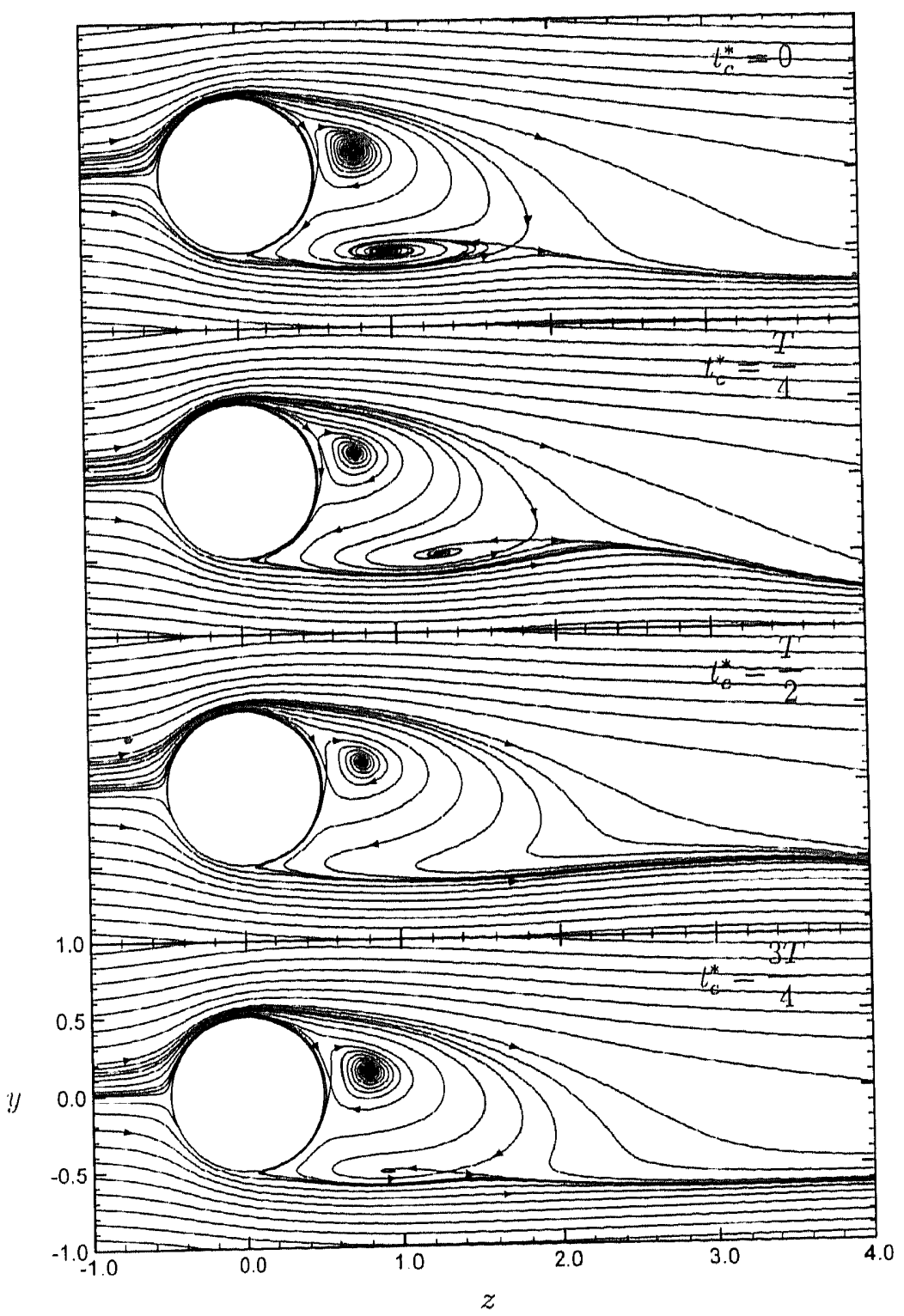

Figure 5.17: Temporal evolution of streamline pattern on the $(y, z)$-plane for $R e=250$ and $\Omega^{*}=0.10$. 


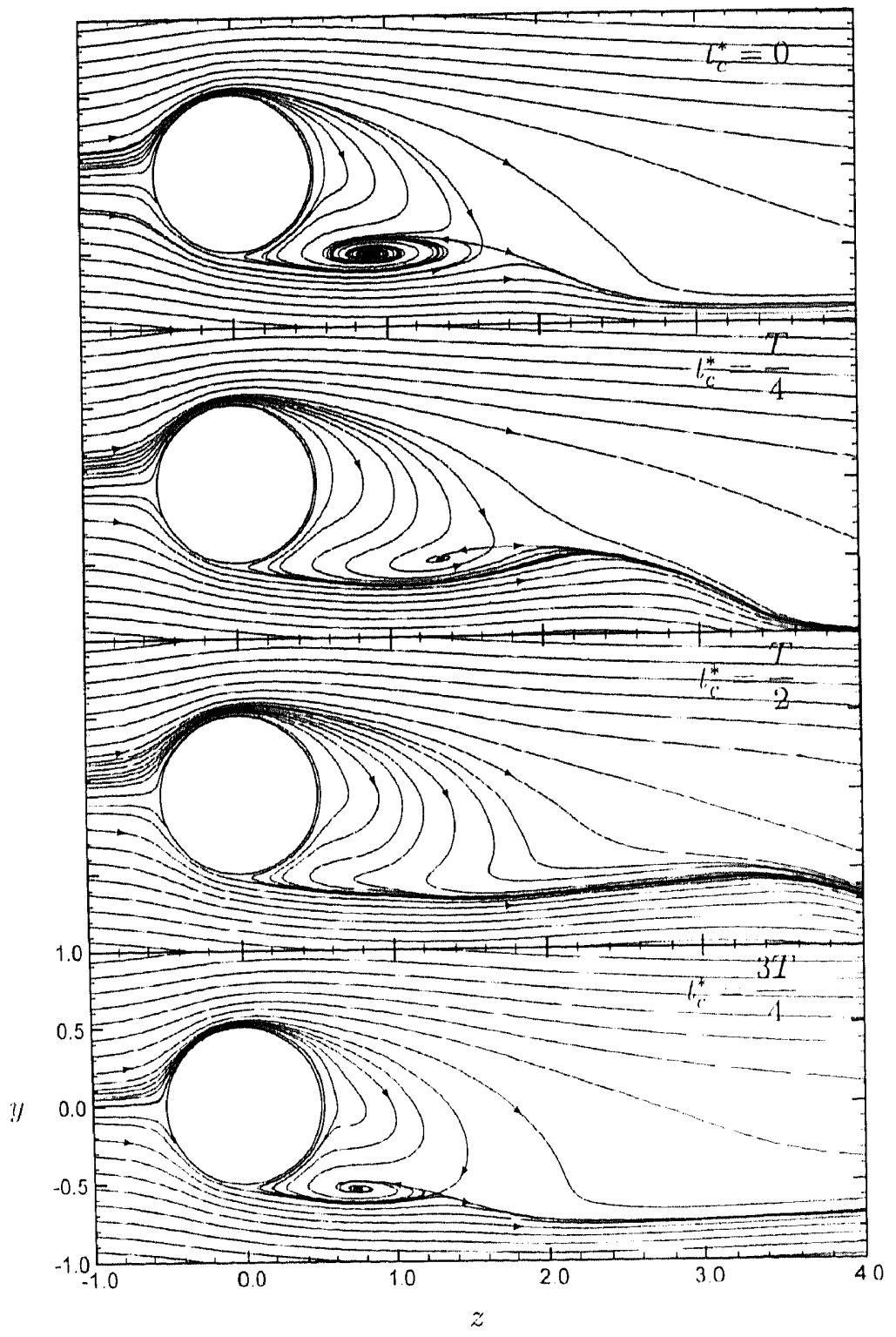

Figure 5.18: Temporal evolution of streamline pattern on the $(y, z)$-plane for $R e=250$ and $\Omega^{*}=0.20$. 
downstream. For $\Omega^{*} \geq 0.50$, all spiraling structures in the wake are absent and the flow appears to remain attached to the surface. As also found for $R e=250$, this topological transition coincides with the onset of vortex shedding suppression. Best (1998) also found wake suppression near $\Omega^{*}=0.50$ for a similar range of Reynolds numbers. In his study, wake suppression was considered to occur when the wake length reduced to zero (where the wake length was defined as "the centreline distance from the downstream edge of the sphere to the furthest downstream point of flow revorsal within the wake") In the present study it was felt that, for a rotating body, this is not a sufficient criteria. to judge the existence of a recirculating wake. The inadequacy of this criteria is demonstrated in figure 5.20, which shows the streamline pattern for $R e=100$ and $\Omega^{*}=0.30$, together with lines of zcro streamwise velocity, $u_{z}=0.0$, and wall-normal velocity, $u_{r}=0.0 . \dagger$ Although the strcamline pattern suggests the wake to be suppressed, the streamwise and radial velocitics indicate a finite reverse flow region. This reverse flow region arises due to the flow following the surface down towards the advancing side of the sphere and is not a reverse flow region in the common sense. Due to a lack of an adequate definition of the wake length for a rotating body, an attempt to quantify the wake length and the rotation rate for wake suppression has been deliberately avoided.

With increasing rotation rate, the recirculating wake volume is reduced until fluid passing over the advancing side of the sphere remains attached to the lee side surface. Under these conditions the process of build-up and release associated with the vortex shedding cycle is inhibited. The reduction in the circulating wake volume may also explain the increase in the vortex shedding frequency with rotation rate. $f$ Naively, the build-up and concentration of vorticity should occur at a faster rate as the wake volume of the recirculating region is reduced. An increasc in the entrainment of freestream fluicl may also drive the shedding cycle at a faster rate.

$\dagger$ In the study of Best (1998) it is not made clear whether the reverse flow region is defined based on streamwise velocity or wall-normal velocity, so both are presented in figure 5.20.

$\ddagger$ In $\S 5.1$, an increase in the vortex shedding frequency was inferred from the progressive reduction in the streamwise distance between successive wake structures. 
As the rotation rate is increased further, the fluid passing over the retreating side of the sphere becomes increasingly vectored in the negative $y$-direction, while the streamline pattern is topologically unchanged. In $\S 5.1$, it was found that for $\Omega^{*} \geq 0.80$ the wake undergoes a further transition to a sccond vortex shedding regime. This transition is not marked by a noticcable change in the near-wake streamline pattern. Unlike the shedding cycle at lower rotation rates, here the shedding mechenism is cloarly not, one of build-up and release of vortical structures from the near-wake. Although unstcacly spiraling structures exist further downstream, in a reference frame translating with the sphere, they appear mercly as a waviness in the downstream strcamlines.

For the cases $\Omega^{*}=0.00,0.30$ and 0.80 , time sequences of the instantineous streamline evolution on the $(y, z)$-plane are presented in figures 5.21, 5.22 and 5.23, respectively. For $\Omega^{*}=0.00$ the instantancous near-wake at the start of the cycle comprisces an unstable focus on the upper side of the sphere. Instantaneous streamlines spiral raclially away from this upper focus before crossing to the lower side of the near-wake and spiralling towards the lower stable focus. The lower focus also instantunomsly cutrains fluid from the lower side of the sphere and grows rapidly. At the next cluarter perioxl, fluid instantancously expelled from the upper focus encireles the lower forus before moving downstream. The lower focus is displaced further clownstrenm from the sphere and has changed from having a stable to motable foral topology. The forms is encirelerl by a limil cycle, onto which streamlines converge from both sides. Johnson \& Patel (1999) noted that the appearance of the limit cycle demarcates the shift of the focus from stable to unstable topology. For a nominally two-dimensional hluff besty, Steiner (1984) found that during the shedding cycle, the change of stable foci to mustable foci was consistently accompanied by the appearance of a limit cycle. Al $l_{c}^{*}=T / 2$ the limit cycle is no longer present and the lower focus is now unstable. The? upper focus has changed from an unstable to stable topology and instiantancously contrains fluid from the upper side of the sphere. At the last quarter period the lower focus has moved further downstrcam and loses its spiral appearance in a stationary reforence 


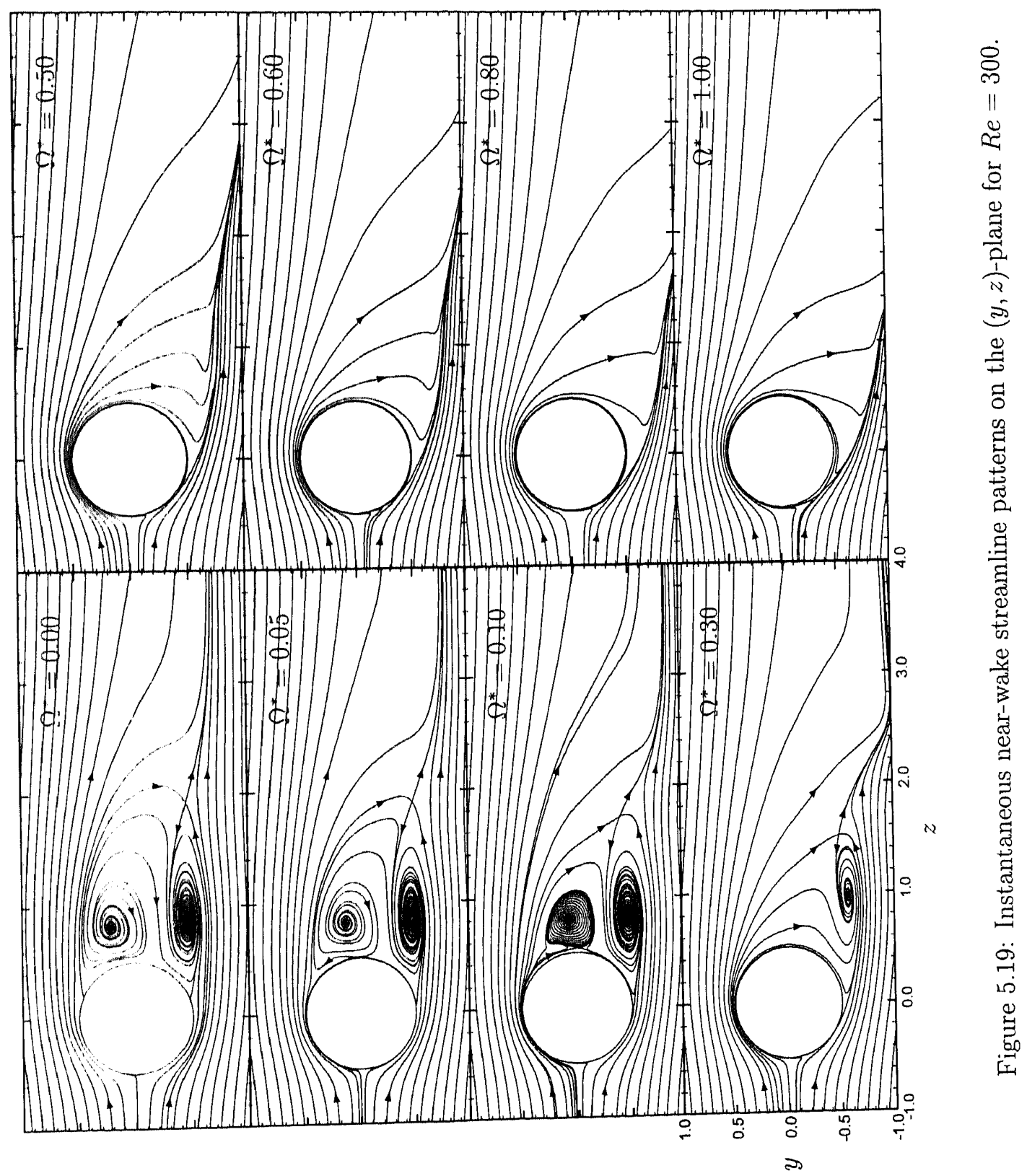




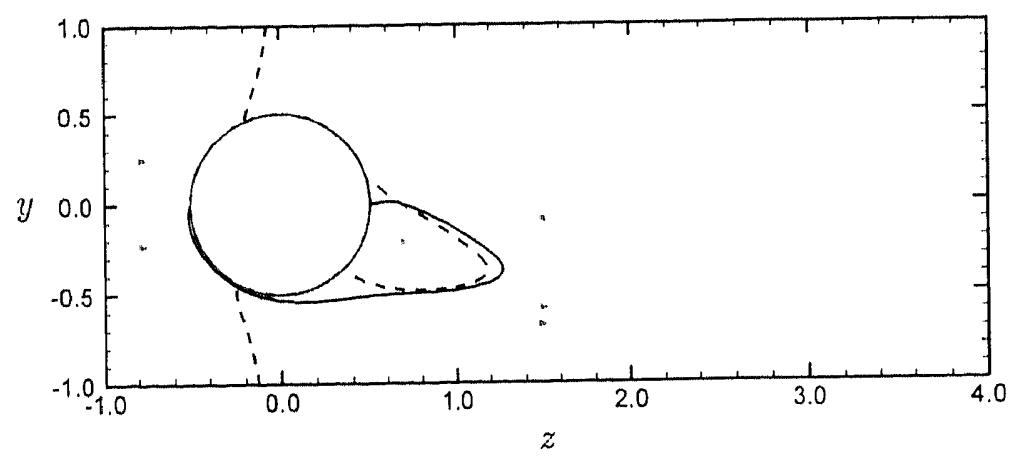

Figure 5.20: Streamline pattern and reverse flow region for $R c=100$ and $\Omega^{*}=0.30$. Streamlines are indicated by solid grey lines. The boundary of the reverse flow region, as identified by the line $u_{z}=0.0$ and $u_{r}=0.0$, is indicated by solicl black and dashed black lines, respectively.

Crame. $\Lambda$ recontly formed lower focus appears in line with the upper focus. This new structure is stable and is fed by fluid entrained from the upper side of the sphere. The upper focus is unstable and expels fluid which encircles the lower focus before being convected downstream. Overall, the shedding cycle is one of 'build-up and release' of the lower focus.

For $\Omega^{*}=0.30$, the near-wake evolution presented in figure 5.22 is similar to that shown in figure 5.18 for $R c=250$ and $\Omega^{*}=0.20$ and is presented here without further description. It should be noted however, that, despite distinctly different flow structures for a stationary sphere at, $R e:=250$ and $R e=300$, the forcing (he to sphere rotation drives the wake to a similar state.

For $S \Omega^{*}=0.80$, the near-wake streamline patterns show little variation over the shexdling period. In the near-wake, fluid passing over the retreating sicle of the sphere remains attached over the lee side of the sphere until it mects fluid passing of the advancing side and loaves the surface to form a free shoar layer. To reveal the wake structures (as identified in $§ 5.1$ ), the velocity field must be viewed relative to an observer moving close to their convection velocity. For a wake flow, the structures develop with streamwise extent and are not periodic in space, therefore a unique convection velocity 
does not, in general, exist. In figure 5.23, the streamlines in the top pancl are overlayed with grey scalc contours of $\lambda_{2}$ in the range -0.0008 to -0.34 . The velocity of the observer was chosen as the convection velocity of the vortical structure centered at $(x, y, z)=(0.000,-2.378,11.554)$, at which point the local velocity is $\left(u_{x}, u_{y}, u_{z}\right)=$ $(0.000,-0.093,0.821)$. The center of the structure was assumed to coincide with the local minimum in $\lambda_{2}$.

In the chosen reference frame, the streamline pattern shows a vortex shect curlingup into a serics of stable foci that are scparated by saddles. In the first pancl, the locations of the foci coincide with the locations where the hairpin structures, as identified by the $\lambda_{2}$ definition, intersect the symmetry plane. With downstream distance, the waviness in the vortex sheet grows until fluid is observed to roll-up into foci. In the third pancl, the initial roll-up of the shear layer is evident near $z=5.0$. Over the shodding cycle the focal structures are seen to grow with streamwise distance, thus entraining more of the surrounding fluid.

Above the chain of stable foci, a positive open bifurcation line exists, from which streamlines on either side diverge. $\dagger$ The downstream streamline pattern bears a striking resemblance to the phasc-averaged strcamline patterns presented by Perry et al. (1980) and Pcrry \& Tan (1984) for a negatively buoyant wakc. From continuity arguments, fluid entrained into the stable foci is ejected from either side of the symmetry plane, while fluid must converge towards the positive open bifurcation line from either side of the symmetry plane. Although the experiments of Perry et al. (1980) were limited to field measurements on the flow symmetry plane, continuity arguments allowed them to conjecture the streamline pattern in a cross-flow plane. Figure 5.21 shows the streamline pattern computed from the in-plane velocity vectors projected onto the cross-flow plane at $z=11.5$, together with the contours of $\lambda_{2}$. The plane of interest is indicated in the top panel of figure 5.23. The computed streamline pattern is in agree-

$\dagger$ A negative bifurcation line is defined as that which streamlines asymptote towards from cither side. 
ment with those conjectured by Perry et al. (1980). The bifurcation line apparent on the symmetry plane forms part of a strcam-surface, which in the cross-flow plane, appears as a scparatrix strcamline. The cross-stream pattern is characterized by a pair of counter-rotating foci which entrain fluid from below the separatrix streamline and produce a downwash on the symmetry plane. The cross-flow plane cuts through the head of a hairpin structure and as a result the contours of $\lambda_{2}$ take a kidney shape. The streamline pattern is consistent with the formation of trailing vortices, as described in $\S 5.1$.

Figure 5.25 presents time-averaged wake profiles on the flow symmetry plane for the throe Reynolds numbers simulated. The downstream station at, $z=2.5$ was chosen to allow some comparison with the LDA measurements of Best (1998) at the same station $\dagger$ Both the streamwise velocity, $\overline{u_{z}}$, and transverse velocity, $\overline{u_{y}}$, are shown for a number of representative rotation rates. For all Reynolds numbers, the volocity minimum in the stroamwise velocity profile secs a progressive displacement, to the arlvancing side of the sphere as the rotation rate is increased. For $R e=100$ there is also a marginal increase in the velocity minimum. This behavior is consistent with that observed in the wake profiles presented by Best for $R e \approx 74,143$ and 206 . For $R e=250$ and $R e=30()$, the velocity minimum shows a more marked increase 110 to $S 2^{*} \approx 0.50$, before decreasing again. It is conjectured that this behavior is associated with the musteady nature of the wake at these Reynolds numbers, which results in a groater entrainment and mixing in the near-wake and therefore a lower wake deficit. A similat behavior is not reflected in the transverse velocity profiles, which for all Reynolds numbers show a progressive decrease and displacement of the velocity minimum to the advancing side of the sphere.

The effect of Reynolds number is shown more clearly in figure 5.26, where the time-averaged wake profiles for $\Omega^{*}=0.00,0.30$ and 0.60 are presented. The wake.

$\dagger$ In the study of Best (1998), the coordinate system is centered at the downstream pole of the sphere and the streamwise and transverse axes are $x$ and $y$, respectively. Measuremonts ure taken nt, $x / d=2.0$, which in the present study corresponds to $z=2.5$. 
profile measurements of Best at the same rotation rates are also shown. Although the Reynolds numbers considered by Best are different to those simulated in the presented study, his measurements at Re $\approx 74$ and 143 are expected to bound the numerical data at $R e=100$, at lcast for a stationary sphere. For $\Omega^{*}=0.00$ the velocity profiles of Best show little variation with Reynolds number and are similar to the numerically simulated profiles at $R e=100$. It is well known that bolow the regular transition, the recirculation region shows a progressive streamwise growth with Reynolds number and it is not clear why this behavior is not evident in the wake profiles measured by Best. For the numerical simulations, an increase in Reynolds number from $R e=100$ to $R e=250$ shows an increase and a downward displacement of the streamwise velocity minimum, while at $R e=300$, the onset of unsteadiness results in a decrease in the velocity minimum. For $\Omega^{*}=0.30$ and 0.60 , the streamwise velocity profiles of Best show the velocity minimum to become displaced towards the advancing side of the sphere with decreasing Reynolds number, while the numerical results show the opposite trend. In both studies, it is evident that the velocity gradient below the velocity minimum becomes more pronounced at higher Reynolds number, which is indicative of the intensification of the shear layer on the advancing side of the sphere. This is shown more clearly in the subsequent section where the vorticity field is presented. 


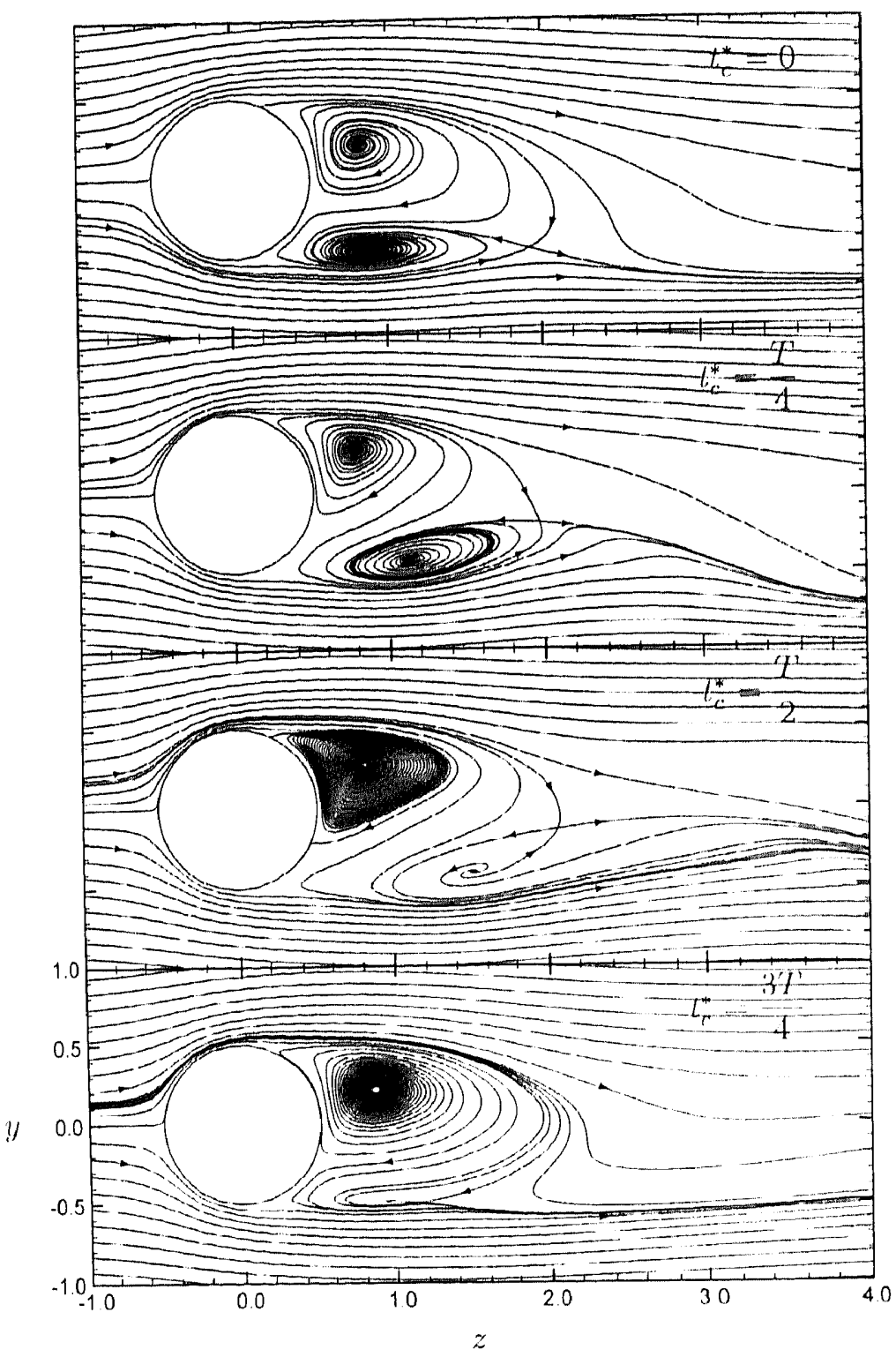

Figure 5.21: Temporal evolution of streamline pattern on the $(y, z)$-p lane for $R e=300$ and $\Omega^{*}=0.00$. 


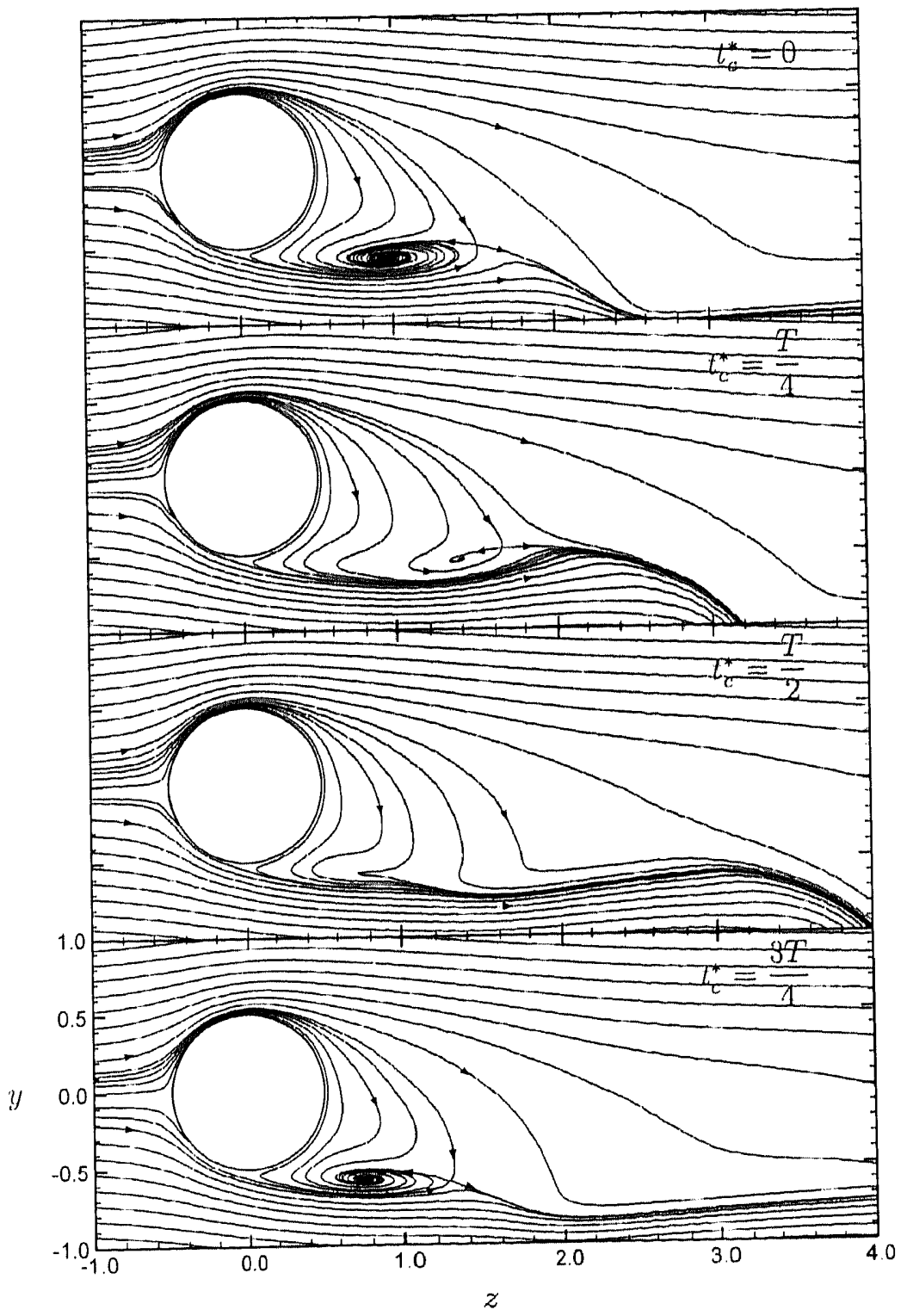

Figure 5.22: Temporal evolution of streamline pattern on the $(y, z)$-plane for $R e=300$ and $\Omega^{*}=0.30$. 


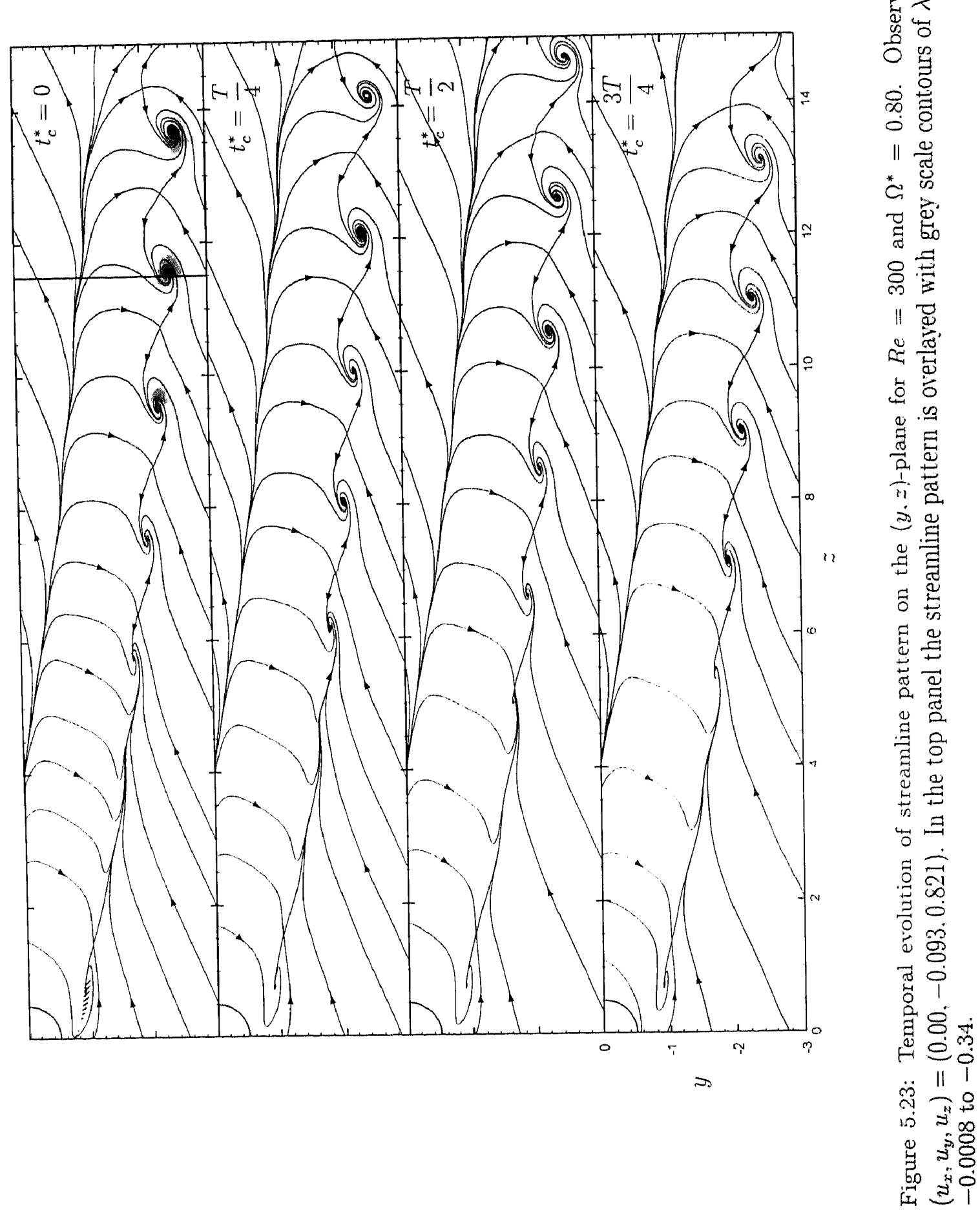




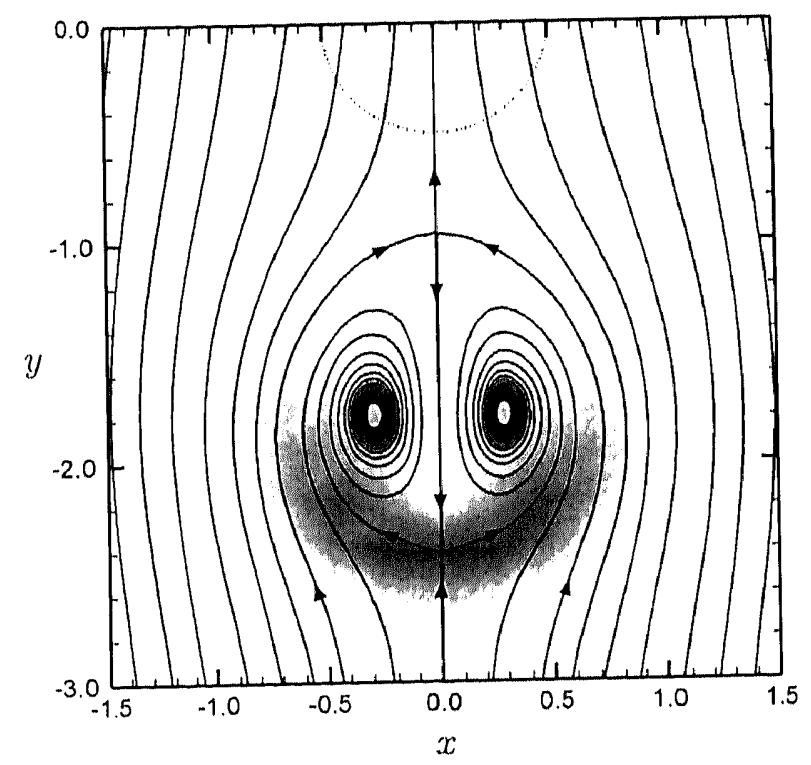

Figure 5.24: Instantancous strcamline pattern on the cross-stream plane at $z=11.5$. $\Lambda$ lso shown are grey scale contours of $\lambda_{2}$ in the range -0.0008 to -0.34 . The time instint shown corresponds to $t_{c}^{*}=0$ in figure 5.23 . 

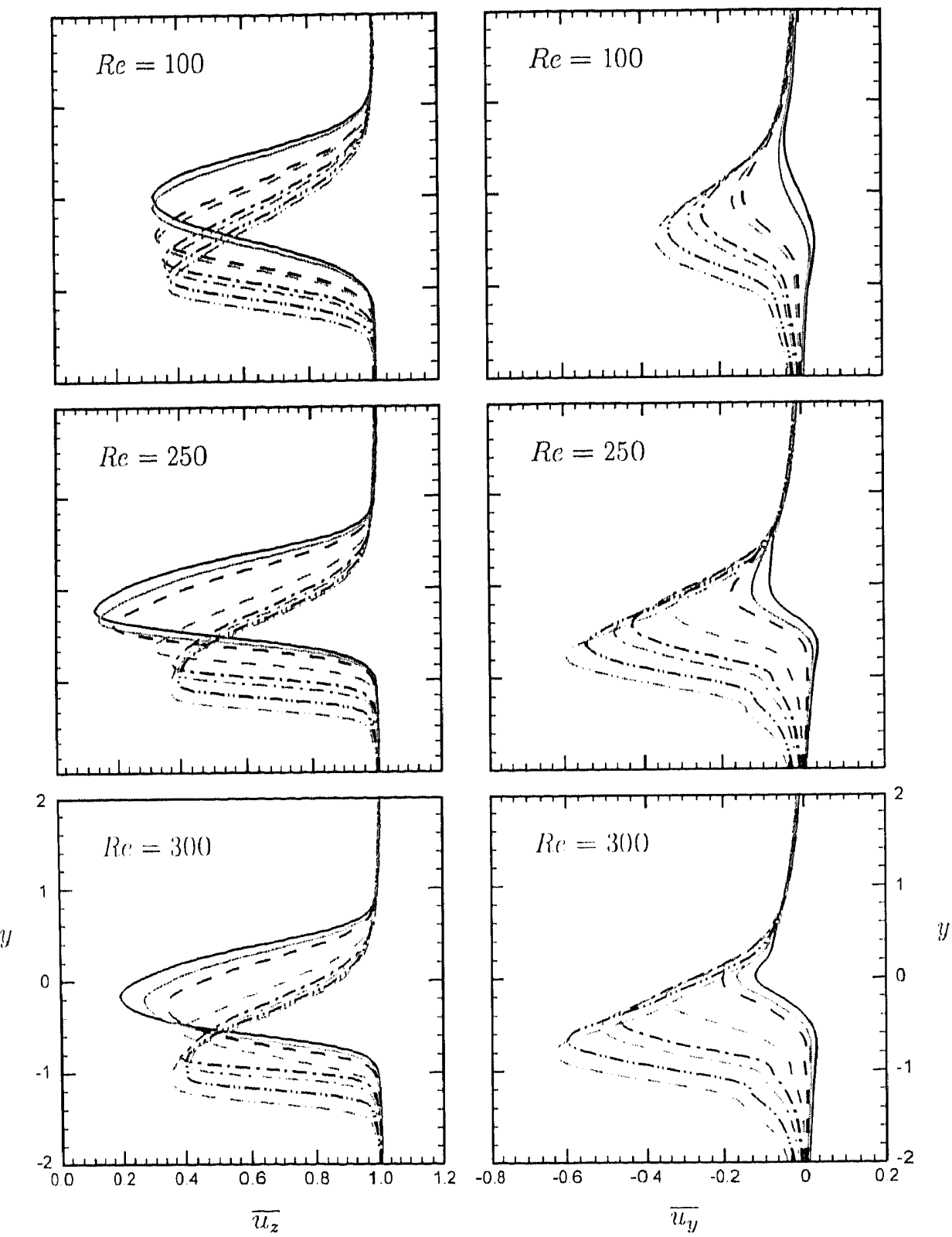

Figure 5.25: Profiles of $\overline{u_{z}}$ and $\overline{u_{y}}$ at $z=2.5$ for Re $=100,250$ and 300 at, a number of representative rotation rates:,$- \Omega^{*}=0.00 ;-, \Omega^{*}=0.05 ;-\cdots, \Omega \Omega^{*}=0.10$; $\cdots \cdots, \Omega^{*}=0.30 ;-\cdots, \Omega^{*}=0.50 ;-\cdots, \Omega^{*}=0.60 ;-\cdots-, \Omega^{*}=0.80 ;-\cdots-$, $\Omega^{*}=1.00$. 

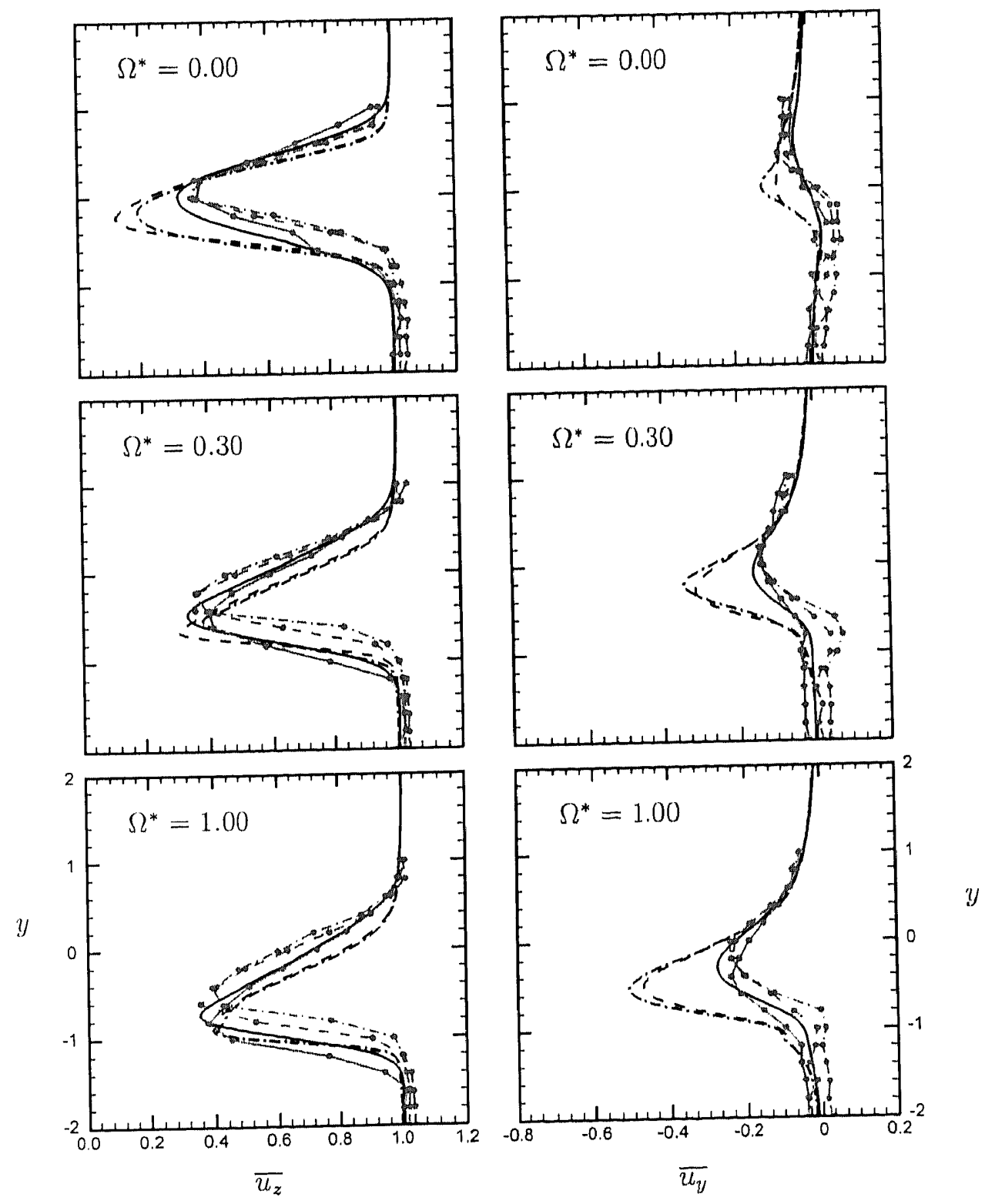

Figure 5.26: Profiles of $\overline{u_{z}}$ and $\overline{u_{y}}$ at $z=2.5$ for $\Omega^{*}=0.00,0.30$ and 1.00. Prosent results:,$- R e=100 ;-\cdots, R e=250 ;-\cdots, R e=300$. Bost (1998): $-\longrightarrow$, $R e \approx 74 ;--; R e \approx 143 ; \cdots-, R e \approx 206$. 


\subsection{Vorticity distribution}

Owing to the threc-dimensional nature of the flowfield, the vorticity vector field comprises three non-zero components. For the sake of brevity, only those aspects which most effectively promote the understanding of the nature of the wake are presented. The $x$-vorticity field on the $(y, z)$-plane for $R e=100$ and a number of representative rotation rates is presented in figure 5.27. Since the $(y, z)$-plane coincides with the flow symmetry plane, only $\omega_{x}$ is non-zero on the plane. In these and subsecquent figures which show the $x$-vorticity ficld, the contours range from -20 to 20 in increments of 0.5 , with dashed lines used to indicate negative values and the zoro contour omitted for clarity. For a stationary sphere, the wake is axisymmetric and the vorticity field comprises two shear layers of equal magnitude and opposite sign. The presence of the toroidal wake, as identified in the noar-wake streamline patterns, is not, apparent in the vorlicity contours. As was mentioned in chapter 4, vorticity fails to distinguish between rotation resulting from a swirling motion and rotation due to shear. Here the cylindrical shoar layer released from the surface is of a sufficiont magnitude to mask the toroirlal wake structure.

The introduction of sphere rotation increases the surface-to-frecstream velocity gradienterer the advancing side of the sphere and decreases it over the retreating sicke This learls to the intensifieation and thimning of the shear layer released from the advancing side, which is seen to extend further clownstroam with increasing retalion rate. The shear layer over the retreating side is weakened and retracts upstream with increasing rotation rate. It also expands laterally and is drawn down over the lee side of the sphere. The effect is most pronounced on the $(y, z)$-plane where the sphere peripheral velocity is greatest. This is illustrated in figure 5.28, where vortex lines for Re $=100$ and $\Omega^{*}=1.00$ are shown. Here vortex lines constrained to the surface are colored light grey, while off-body lines are colored black. The off-body vortex lines are released from the symmetry plane at equidistant streamwise stations. The $y$-coordinates were taken 
along the line of peak $\omega_{x}$ on the shear layer scparating from the advancing side. For a stationary sphere, an axisymmetric cylindrical shear layer separates from the surface and the vortex lines form a scries of circles that lie parallel to the $(x, y)$-plane (not shown). Sphere rotation distorts and stretches these vortex lines. On the retreating side, the vortex lines are tilted upstream and drawn toward the lee side of the sphere, while on the advancing side they are tilted and drawn downstream. The effect increases as the symmetry plane is approached, such that the closed vortex loops take a kidney shape when viewed in the cross-flow plane.

Returning to figure 5.27, for a stationary sphere the line of flow scparation can be identified by a change in sign of the vorticity at the surface. Downstream of scparation, a pair of anti-symmetric vortex lobes are attached to the sphere lec side. With increasing rotation rate, the negative lobe on the retreating side grows, while the positive lobe on the advancing side diminishes and is absent for $\Omega^{*} \geq 0.30$. This trend is clearly secn in figure $5.34(a)$, where surface profiles of $x$-vorticity on the $(y, z)$-plane are presented for a number of representative rotation rates. Here $\theta$ is measured from the front stagnation point and is positive moving towards the retreating side of the sphere. For a stationary sphere, the vorticity profile is anti-symmetric about $\theta=0$, with vorticity peaks near $\theta= \pm 55^{\circ}$ and a sign change occurring noar $\theta= \pm 128^{\circ}$. With increasing rotation rate the vorticity minimum on the advancing side decreases and is shifted towards smaller $\theta$. The vorticity peak downstream of separation is reduced, such that for $\Omega^{*} \approx 0.30$ it osculates the $\omega_{x}=0$ line and for higher rotation rates there is no sign change in the vorticity over the advancing side. It should be noted that, unlike a stationary sphere where a sign change in the surface vorticity coincides with a separation or stagnation point, this definition docs not apply to rotating bodics. On the retreating side, the vorticity peak is reduced and shifted downstream. The point at which the vorticity changes sign and the vorticity profile downstream of this point are relatively insensitive to sphere rotation. 
The wake vortex skeleton is found to be best represented by the streamwise vorticity. Figure 5.29 presents iso-surfaces of $z$-vorticity for the same rotation rates considered in figure 5.27. In this and subsequent figures which show $z$-vorticity iso-surfaces, dark and light grey iso-surfaces correspond to threshold values of $\omega_{z}=-0.10$ and 0.10 , respectively. To indicate length scale, the wake cross-sections are marked at, streamwise increments of $\Delta z=2$, starting at $z=0$. For a stationary sphere the wake is axisymmetric and the streamwise vorticity is identically zero. Sphere rotation leads to the appearance of a pair of concentrated strcamwise vorticity threads of equal magnitude and opposite sign. On the upstream side of the sphere, patches of vorticity which are of opposite sign to their respective downstream tails also are gencratcel. With increasing rotation rate the vorticity tails extend further downstream, indicaling an increase in their rotational strength, while the upstream vorticity patches grow to form a shroud over the sphere. These wake structures correlate well with those kelentified in $\$ 5.1$ using the $\lambda_{2}$ elcinition.

For $R e=250$, the $x$-vorticity ficld on the $(y, z)$-plane is presented in figure 5.30 . In this and all subsequent figures that present cases for which the flow is periodic, the instant shown coincides with the lateral lift foree being at a local minimum. For a stationary sphere the vorticity field is asymmetric, with the shear layer separating from the lower-side extending further downstream than that on the upper-side of the sphere. $\Lambda$ comparison with the vorticity contours presented by Johnson \&: Patel (1999) show close agrement, (sec chapter 3). For $\Omega^{*} \leq 0.08$, the effect of sphlere rotation is similax to that at, $R c=100$. The shear layer separating from the advancing side extends further downstrean, while that on the retreating side retracts upstream. $\Omega^{*}=0.10$ marks the onset of vortex shedding and this is seen as a waviness in the shear layer separating from the advancing side of the sphere. For $\Omega^{*}=0.20$, the shed structures have greater rotational strength and discrete segments of negative vorlicity are perioclically torn away from the near-wake. The vorticity minimum near $z=7.0$ corresponds closely with the point where a shed hairpin intersects the symmetry plane. For higher rotation 


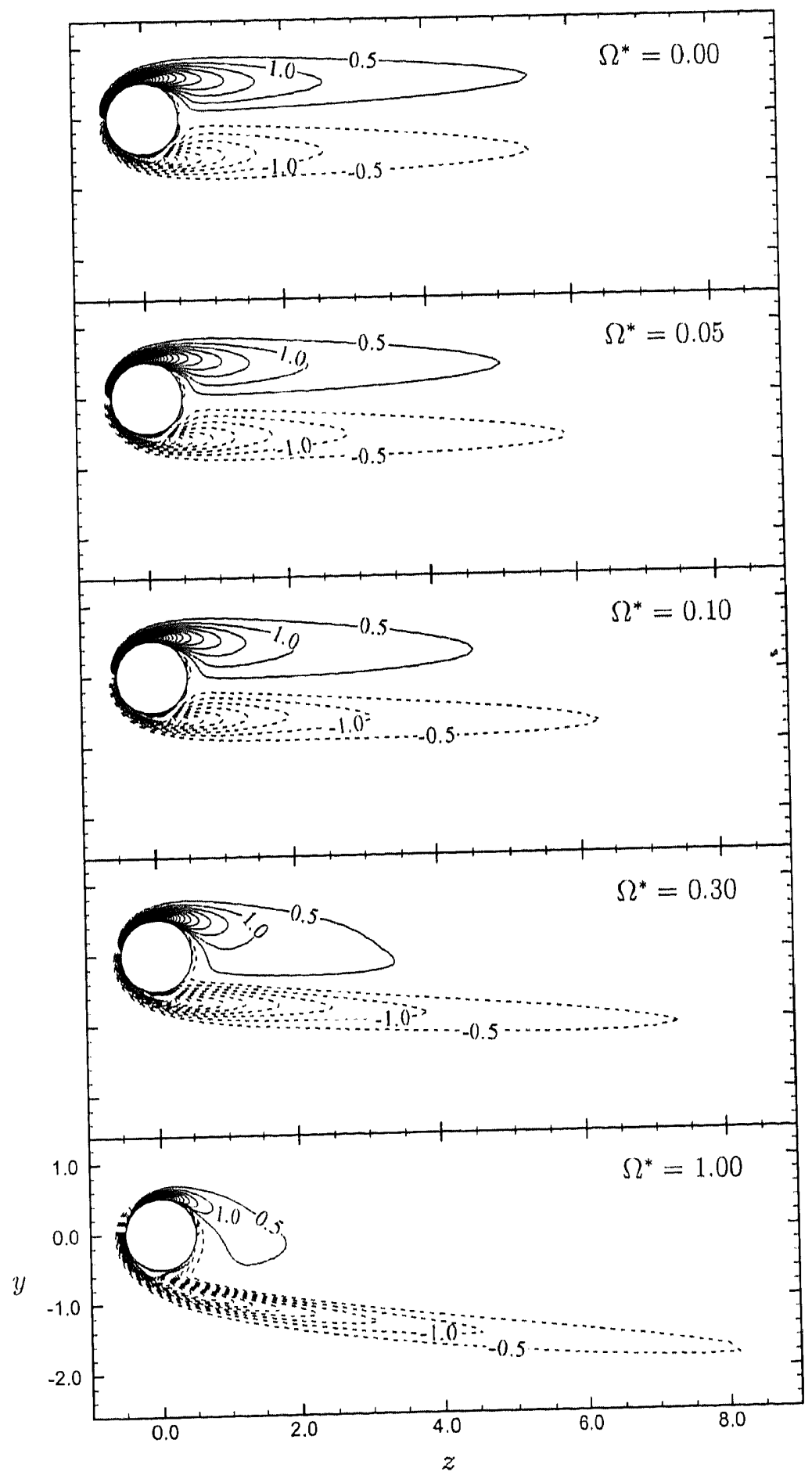

Figure 5.27: Contours of $x$-vorticity on the $(y, z)$-plane for $R e=100$. 


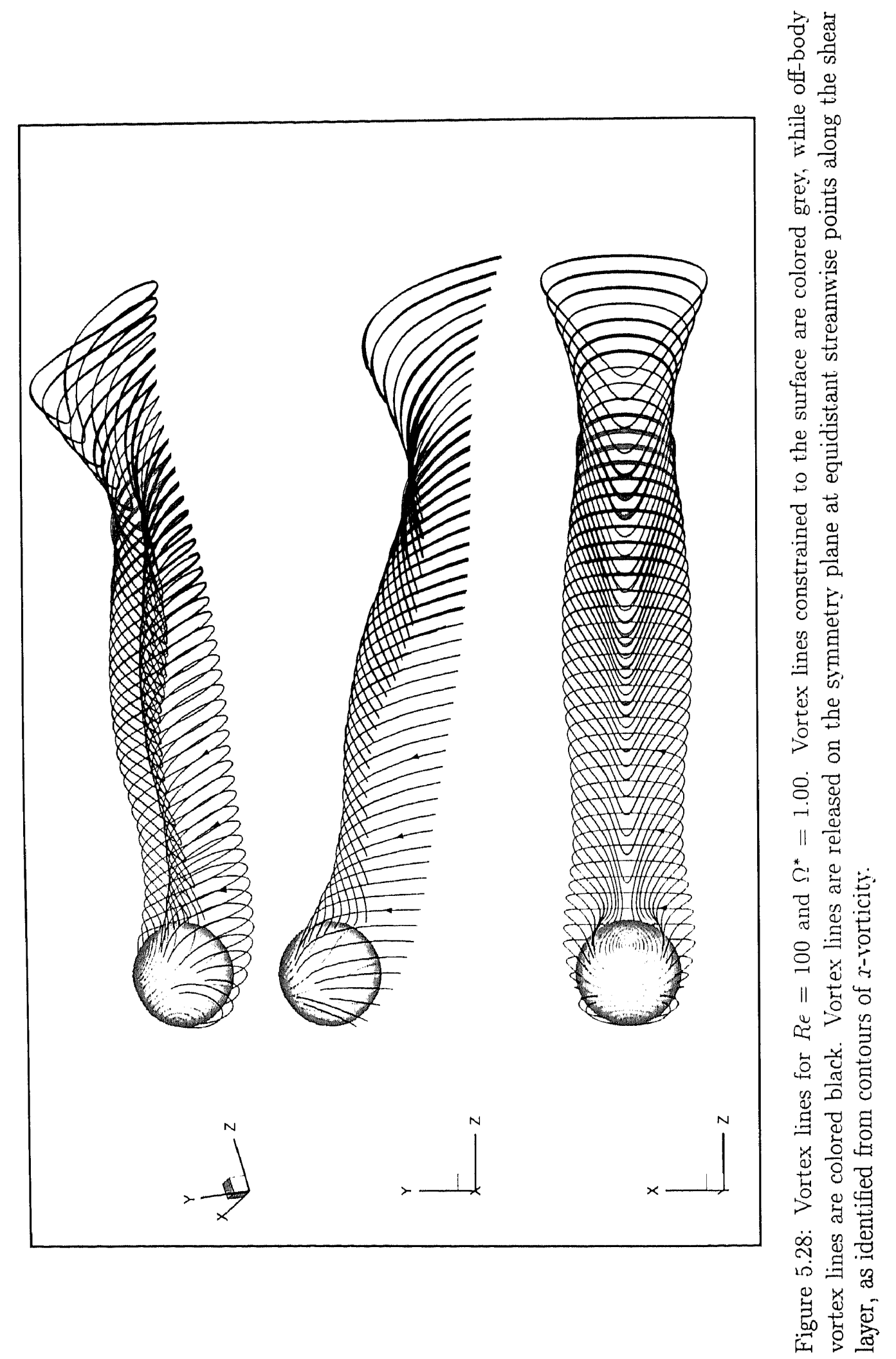




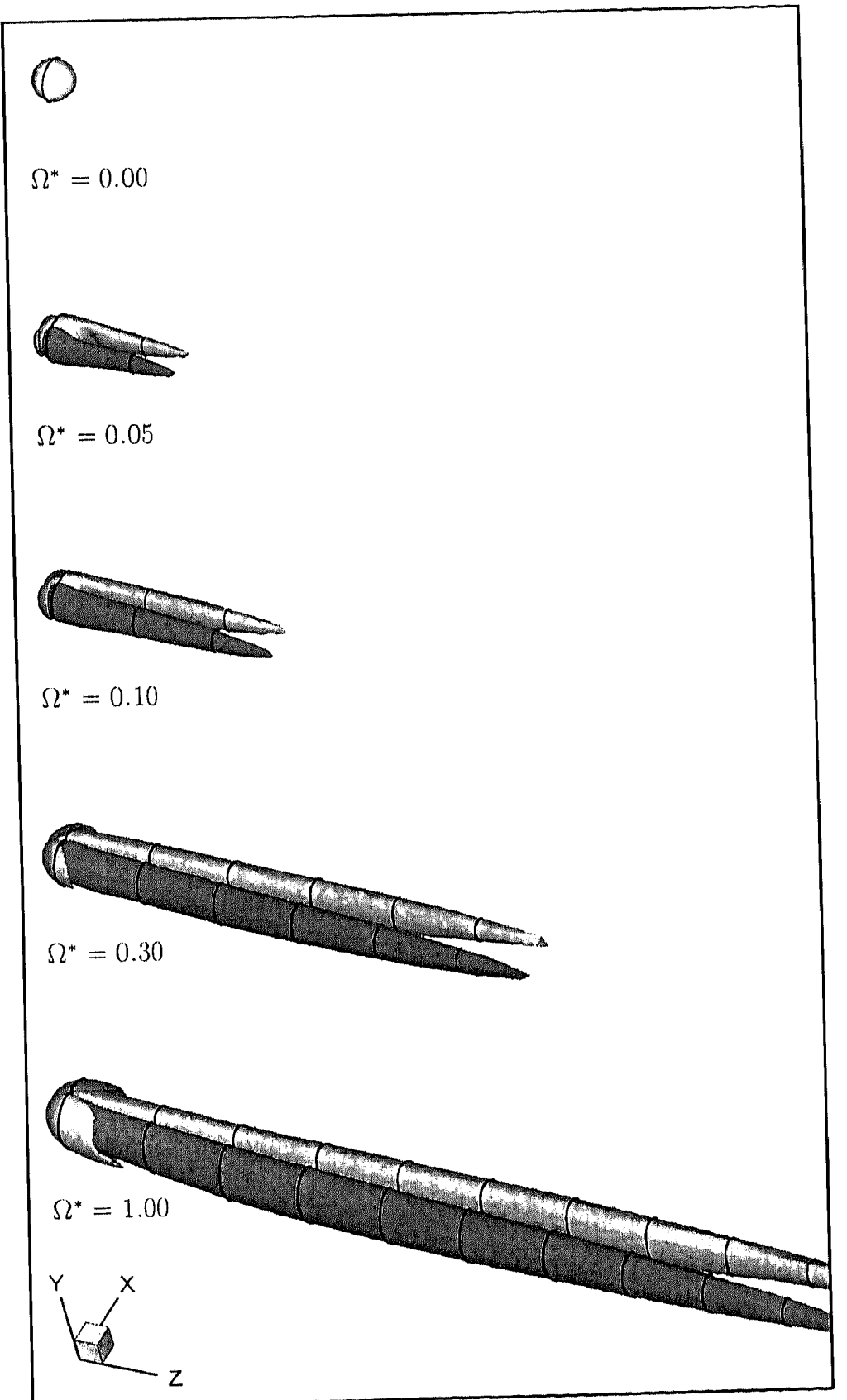

Figure 5.29: Iso-surfaces of $z$-vorticity for $R e=100$. Dark and light grey iso-surfacess correspond to $\omega_{z}=-0.10$ and 0.10 , respectively. 
rates wake shedding is suppressed and the near-wake vorticity ficld resembles that at $R e=100$, although owing to the higher Reynolds number, the shear layer separating from the advancing side is noticeably thinner.

Iso-surfaces of $z$-vorticity are shown in figure 5.31. For a stationary sphere the wake comprises a pair of anti-symmetric streamwise tails. In the near-wake, the streamwise tails are shrouded by vorticity patches of opposite sign. These patches are not linked to the sphere surface and must arise from a tilting of the vorticity generated at the surface. The flow structure compares well with the vorticity iso-surfaces presented by Tomboulides \& Orszag (2000), although in their study the particular values of vorticity magnitude presented are not stated.

The introduction of rotation causes the tails to elongate, while the patches of oppositely signed vorticity in the near-wake are climinated. Instcad, patches of vorticity of opposite sign to the downstream tail appear on the upstroam sicle of the sphere. For $\Omega^{*}=0.08$ the vorticity tails show some waviness. Recall that, at this rotation rate the wake shows small amplitude oscillations with a slow decay rate. The time instant $l^{*}=256.15$ is shown. For $\Omega^{*}=0.10$, the wake undergoes sustatined vortex shedeling and the vorticity iso-surfaces show a series of anti-symmetrie streanwise threads being shed from the sphere and convecting downstrem. On comparison with the structures identified using the $\lambda_{2}$ definition, these threads coincide with the legs of the hairpin which are periodically shed from the sphere with the same orientation. At the upstream (end of the streanwise threads, near $z=2.0$ and $z=6.0$, there are alse patches of vorticity of opposite sign to that of the streamwise threads. Althongh not evident at the threshold value of vorticity magnitude presented, these are connected to the vorticity patches upstream of the sphere and are periodically torn away from their upstrean source and convected downstream.

For $\Omega^{*}=0.20$, the vorticity threads released from the near-wake become kinked midway along their streamwise extent. The patches of oppositely signed vorticily are now larger and, on comparison with the structure identified using the $\lambda_{2}$ definition, it 


\subsection{VORTICITY DISTRIBUTION}

is apparent that these patches evolve to form the legs of the induced hairpin structures, which are of opposite sign and orientation to those structures shed from the sphere. At higher rotation rates, the wake reduces to a steady anti-symmetric pair of concentrated vorticity tails, qualitatively similar to the flow structure found for $R e=100$.

For $R e=300$, the $x$-vorticity on the $(y, z)$-plane is presented in figure 5.32. For a stationary sphere, the shear layer separating from the lower sicle of the sphere is more intense, and it is from this side -... where vorticity is most concentrated that vorticity is periodically discharged downstream. The introduction of sphere rotation leads to the intensification of the sphere layer separating from the advancing side and the patches of vorticity which are periodically discharged downstrcam are noticeably stronger.

For $\Omega^{*}=0.50$, the wake reverts to a steady-state and the vorticity field, on the plane, is characterized by a thin shear layer scparating from the advancing side of the sphere, while that over the retreating side is more diffusc. For $\Omega^{*}=0.80$, the shear layer separating from the advancing side shows some waviness and this precedes it rolling-up to form a scries of concentrated vorticity patches. At the time instant shown, these patches occur near $z=5.5$ and $z=7.5$ and coincide with the locations where shed hairpin structures intersect the symmetry plane. Unlike the structures shed at lower rotation rates, which appear to scale with the diameter of the sphere, these structures scale with the thickness of the shear layer. For $\Omega^{*}=1.00$, the roll-up of the shear layer occurs further upstream and the patches of vorticity released downstrean are stronger. As these structures propagate downstream they grow and become more diffuse. This second transition to vortex shedding works to redistribute the shear layer vorticity, which has become increasingly concentrated as the rotation rate and Reynolds number are increased. These features combine to suggest that the mechanism driving the unsteadiness is a Kelvin- Helmholtz instability of the shear layer separating from the advancing side of the sphere.

Iso-surfaces of $z$-vorticity are shown in figure 5.33. For a stationary sphere, pairs of 
anti-symmetric vorticity threads are periodically released from the surface. These are always shed with the same orientation and with the same sign. As these structures are convected downstream they become kinked near their mid-section. The evolution shows good agreement with the strcamwise vorticity iso-surface presented by Tomboulides \& Orszag (2000); although, in their study, the vorticity magnitude and time instant, shown was not specified. On comparison with the structure identified using the $\lambda_{2}$ definition, it is evident that the location at which the threads kink coincides with the locations at which the shed hairpin structures reconnect to form secondary hairpins. The numerical simulations of Kim \& Choi (2002) also showed the reconnection of the shed hairpin structures, in contrast to the results of Johnson \& Patel (1999) where secondary structures did not emerge.

Patches of vorticity of opposite sign to that released from the surface also appear in the near-wake. These are not connected to the surface and their origin must be similar to the vorticity patches which shroud the streamwise tails for a stationary sphere at, $R e=250$. As these patches are convected downstream they become tilted in the opposite direction to the threads released from the surface and form the legs of the induced hairpin structure, which were first elescribed by Johnsen \& Pated (1999).

With increasing rotation rate the secondary structures are weakened and diffuse quickly, such that they are not discernible in the far-wake. 'The sheed structures become stronger and in the near-wake they are seen to comnect clirectly with stmetures shed at the previons cycle. For $\Omega^{*}=0.50$, the wake reverts to a steady anti-symmetrie pair of streanwise threads and this pattorn persists at $\Omega^{*}=0.60$. The onset of the second vortex shedding regime at $\Omega^{*}=0.80$ shows a series of compact vorticity patches being convected along the streamwise threads, while the noar-wake rescmbles that at, lower rotalion rates, where the wake is steady.

For $\Omega^{*}=1.00$ the shed structures are stronger and the anti-symmetric tails along which they conveyed become separated into discrete patches. Although the contours of $x$-vorticity on the $(y, z)$-plane suggest a shear layer instability, this figurc highlights 
the threc-dimensional nature of the vorticity ficld, which reiterates that the mechanism responsible for the second transition to unsteadiness may not bo simply analogous to that which can occur for a two-dimensional mixing layer.

Finally, the effect of Reynolds number on the time-averaged surface profiles of $x$ vorticity on the $(y, z)$-plane is shown in figure $5.34(b)$. For the three Reynolds numbers considered, the rotation rates $\Omega^{*}=0.00,0.50$ and 1.00 are presented. The effect of increasing Reynolds number is to increase the peak values of vorticity, which rosults from the thinning and intensification of the shear layer. The trend with increasing $\Omega^{*}$ is similar for the three Reynolds numbers. It is also interesting to note that the point of zero vorticity near the forward stagnation point is unchanged with varying $\Omega^{*}$. 


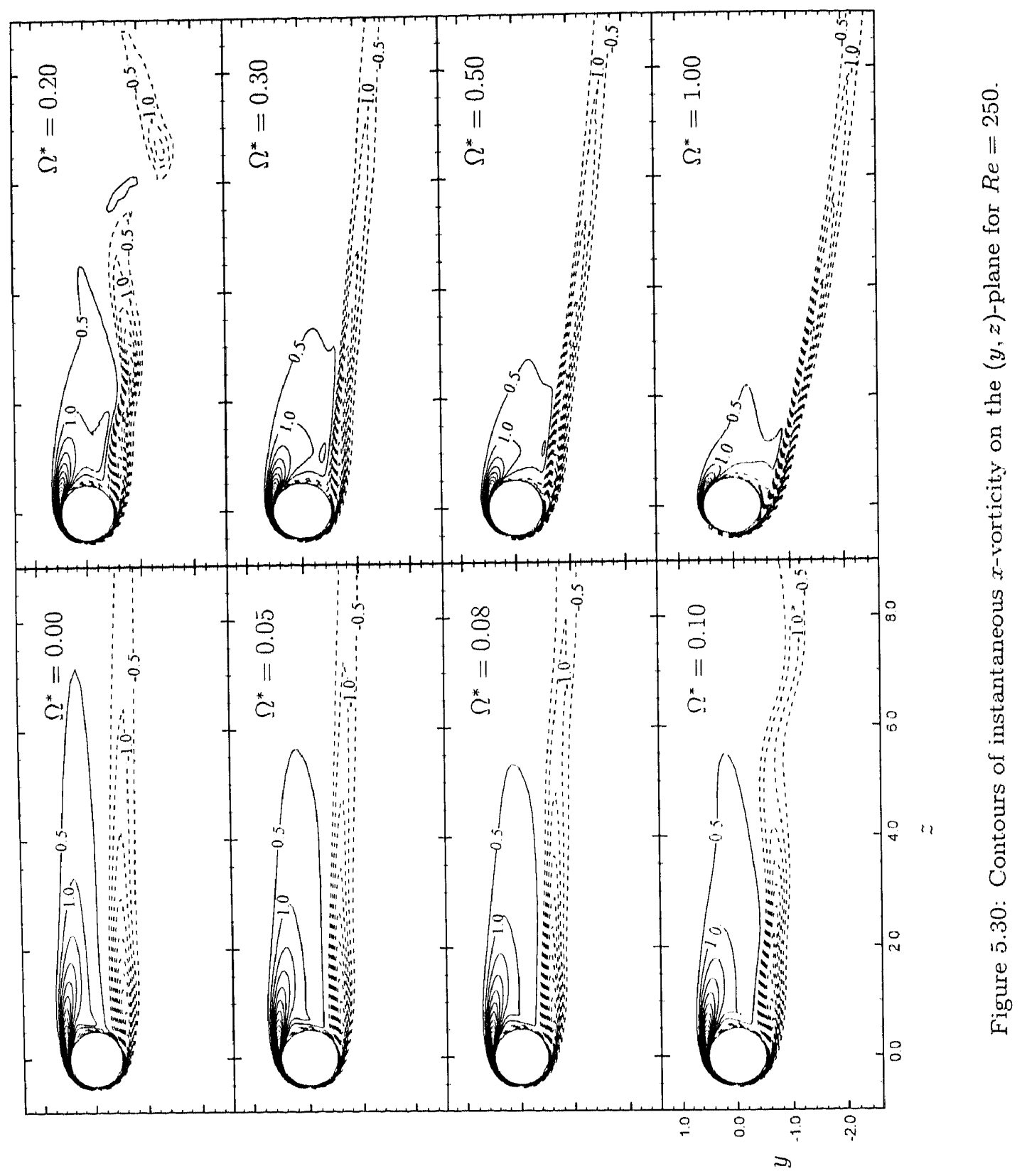


5.3. VORTICITY DISTRIBUTION

177

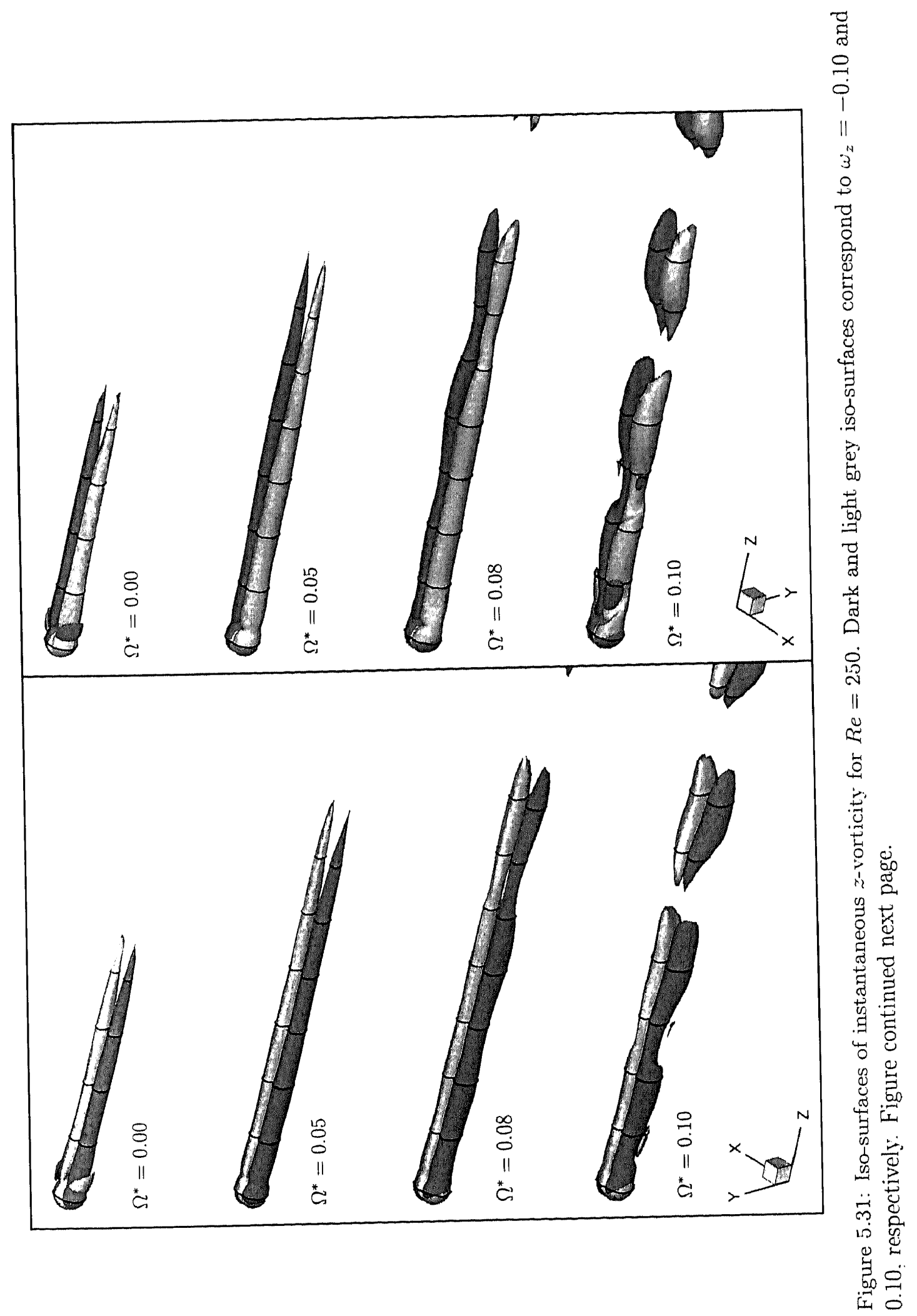




$$
\text { IIII }
$$




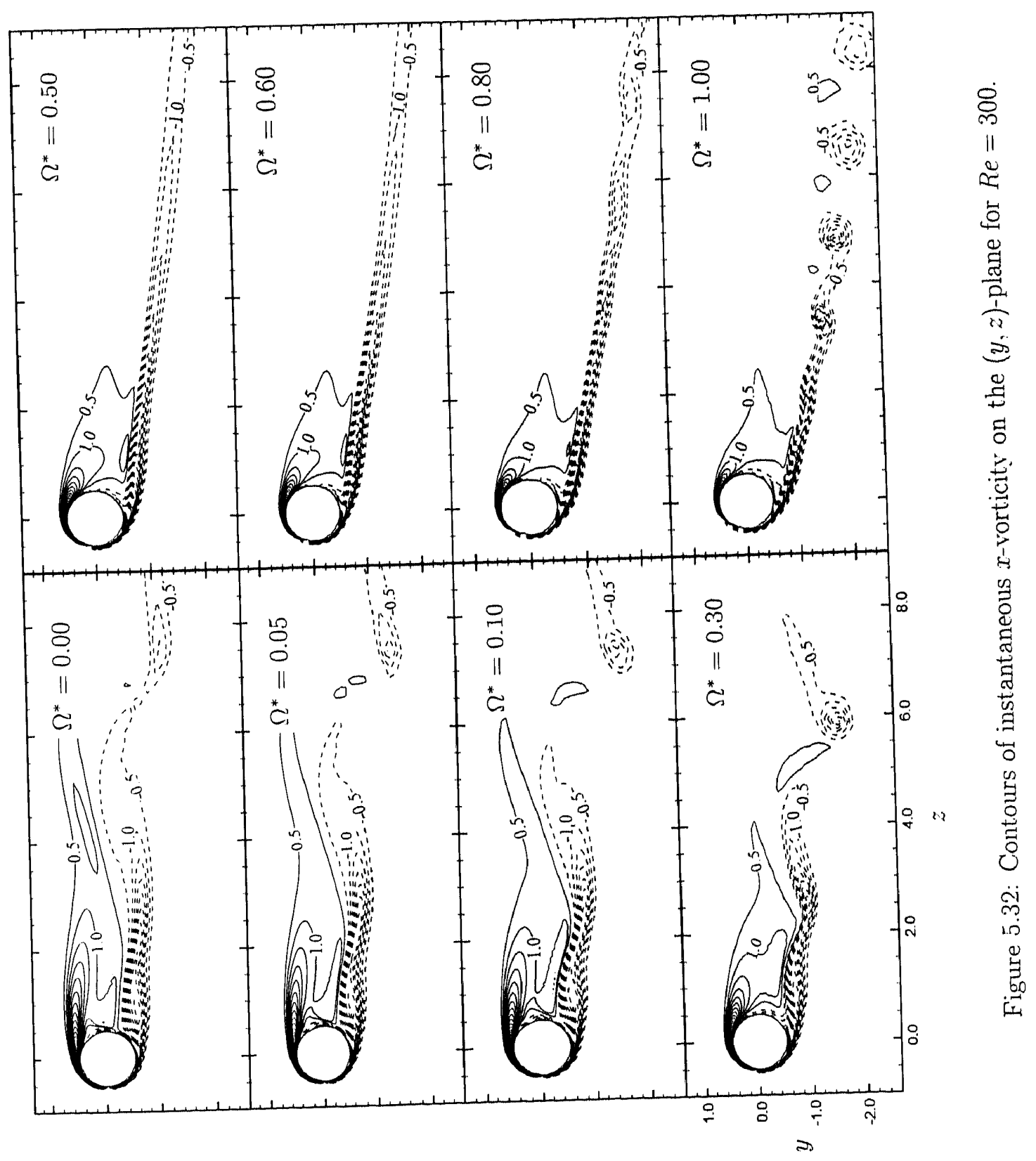



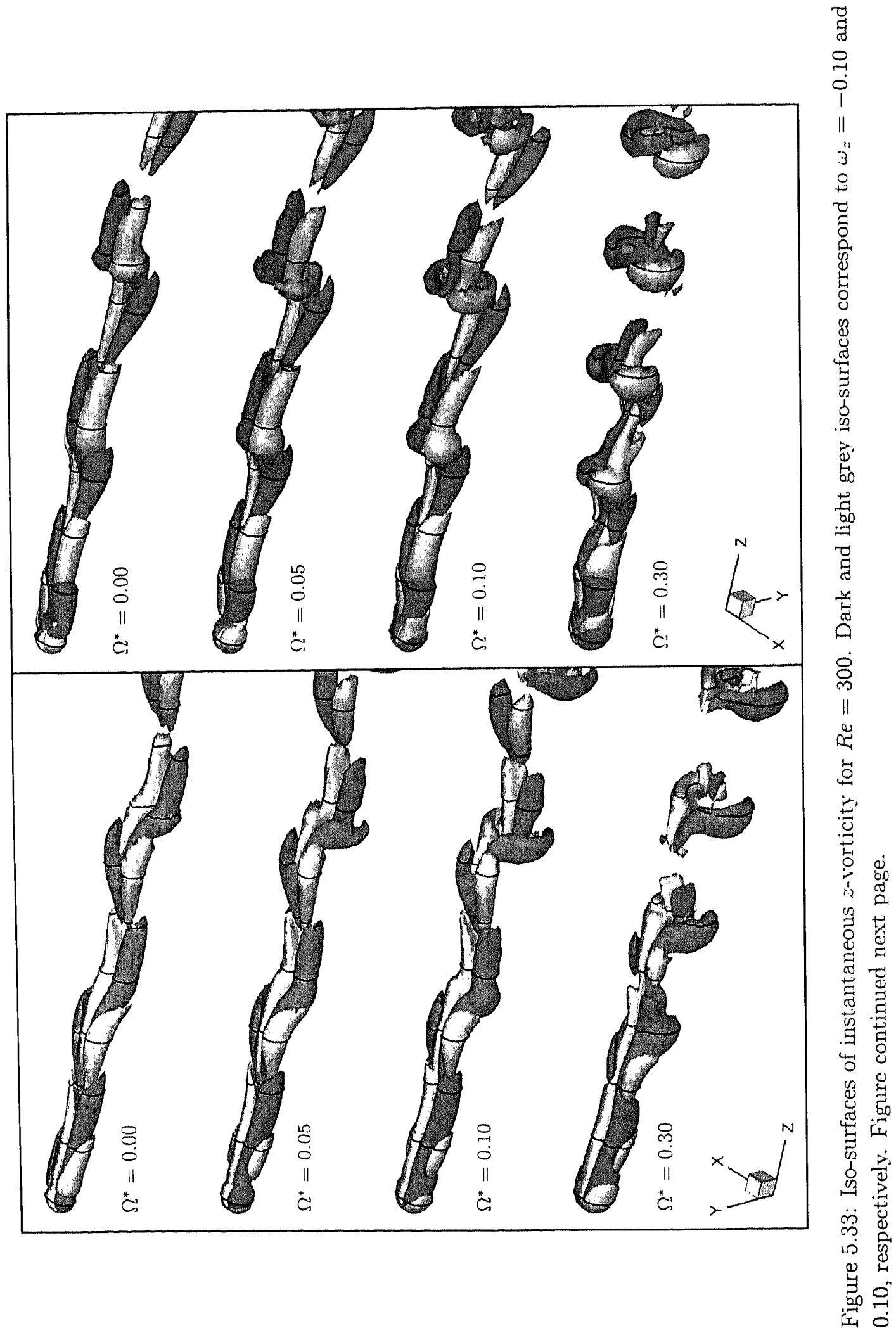


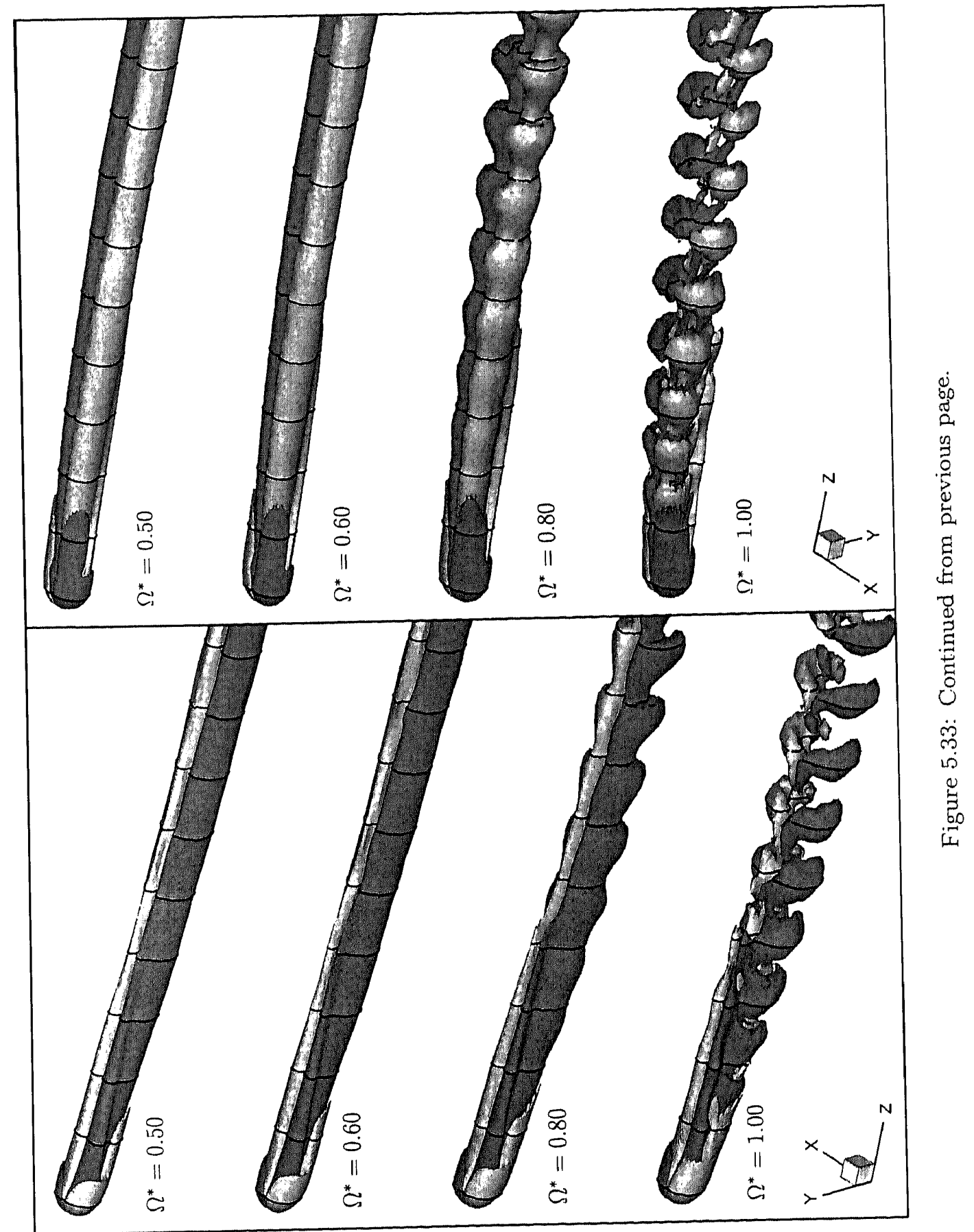



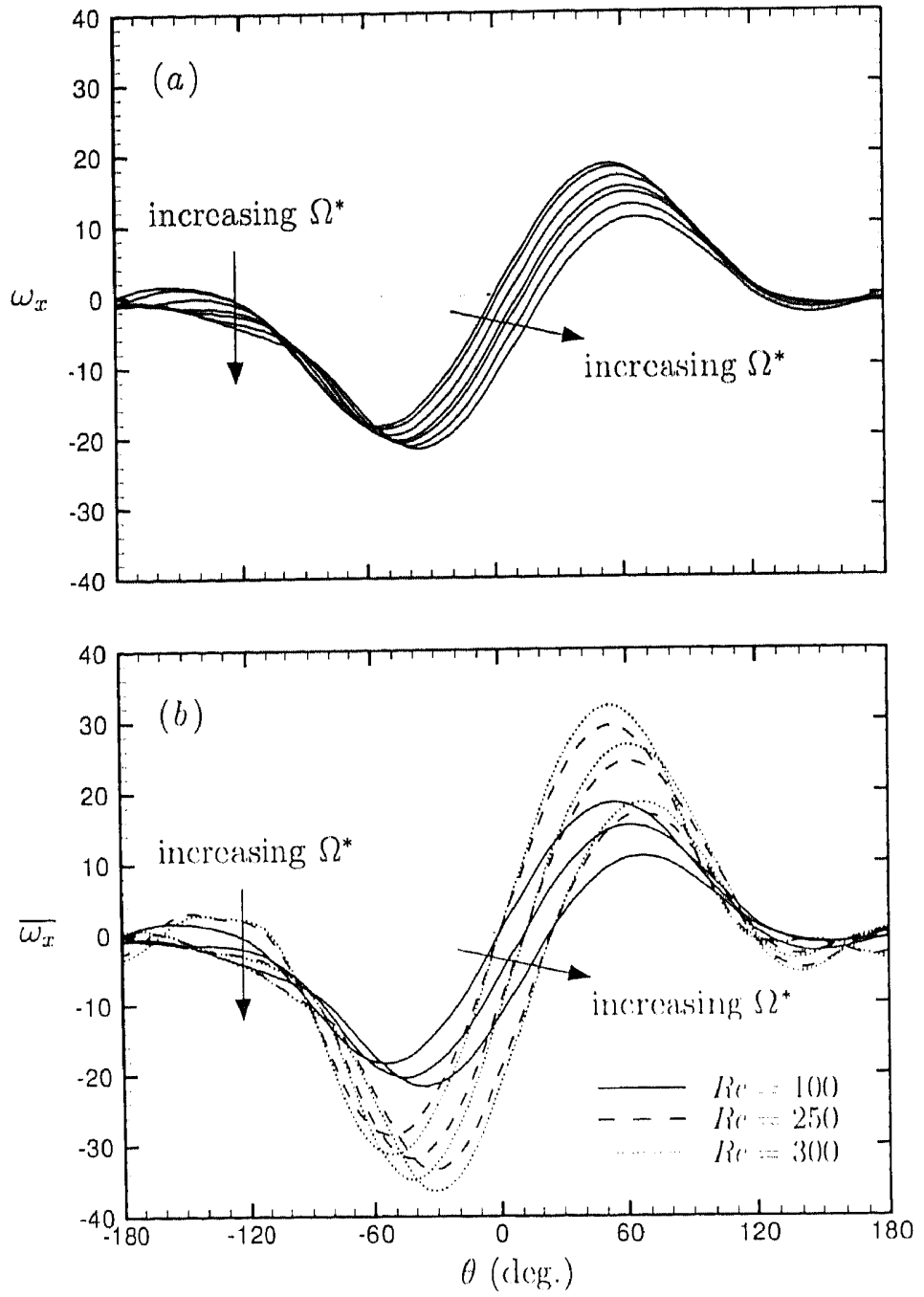

Figure 5.34: Time-averaged surface $x$-vorticity on the $(y, z)$-plane $(\phi=\pi / 2)$ : $(a)$ $R e=100$ and $\Omega^{*}=0.00,0.10,0.30,0.50,0.60,(0.80$ and $1.00 ;(b) R c=100,250$ and 300 and $\Omega^{*}=0.00,0.50$ and 1.00 . 


\subsection{Pressure distribution}

To further examine the nature of the wake, this section describes the spatial distribution of the pressure coefficient. Here the pressure coefficient is defined as

$$
C_{P}=\frac{P-P_{\infty}}{\frac{1}{2} \rho U_{\infty}^{2}} .
$$

Pressure cocflicient contours for $R e=100$ and a number of representative rotation rates are presented on the $(x, z)$-plane in figure 5.35 and on the $(y, z)$-plane in figure 5.36. In these and subsequent figures which show the pressure coefficient field, the contours range -1.30 to 1.10 in increments of 0.04 , with dashed lincs used to indicate negative values. For a stationary sphere the pressure field is in close agrecment with that presented by Johnson \& Patel (1999). The wake is axisymmetric and shows Iwo low pressure lobes on the sphere lee side, on both the $(x, z)$ - and $(y, z)$-planes. In figure 5.12 the streamline pattern shows a closed recirculating wake at this Reynolds number, yet there is no pressure minimum near the axis of the toroidal vortex. Johnson \& Patel found that a pressure minimum appears near $R e=200$. At lower Reynolds numbers the contrifugal accelcration is presumably balanced by viscous forces, rather than a radial pressure gradient. This exemplifies the inadequacy of the pressure ficld as a vortex iclentification method for low Reynolds number flows.

On the $(x, z)$-plane, increasing rotation rate results in a greater suction pressure over the sphere lee side and the symmetric pair of low pressure lobes move towards the downstream pole of the sphere $(\theta=0)$, such that for $\Omega^{*}=0.30$ they coalesce. The reduction in pressure over the lee side is felt as an increase in the pressure induced clrag. Sphere rotation shows a more pronounced effect on the $(y, z)$-plane. With increasing rotation the low pressure lobe over the retreating side moves upstrearn and the pressure is reduced, while the low pressure lobe over the advancing side moves downstream and the pressure over the advancing side increases. It is this pressure difference that is the dominant contributor to the lateral lift force, as will be shown in $\S 5.5$. Despite the near-wake streamline pattern showing a marked change for $\Omega^{*} \leq 0.10$, the effect on the 
pressurc field is less pronounced. For $\Omega^{*} \geq 0.30$ the low pressure lobes coalesce and there is a smoothing of the wall-parallel pressure over the lec side. The smoothing of the pressure ficld coincides with the streamline pattern, presented in figure 5.13, showing the flow to remain attached over the lee side of the sphere. For higher rotation rates there is the appearance of a low pressure streamwise tail which arises from the vortical streamwise tails generating a contrifugal accoleration along their length.

Figure 5.37 presents surface pressure coefficient profiles on the $(x, z)$ - and $(y, z)$ planes for a number of representative rotation rates. Here $\theta$ is measured from the front stiagnation point and on the $(y, z)$-plane it is positive moving towards the retreating side of the sphere. For a stationary sphere, the pressure takes a minimum noar $\theta=90^{\circ}$. On the $(x, z)$-plane sphere rotation shifts the pressure minimum to slighthly higher $\theta$ and there is the appearance of a sccond pressure minimum contered at the downstream pole of the sphere. On the $(y, z)$-plane, the pressure minimum near $\theta=900^{\prime}$ secs a pronounced increase with rotation rate, while the pressure minimum on the retreating side is reduced and is absent for $\Omega^{*}>0.30$. This has the effect of climinating the adverse pressure gradient over the advancing side of the sphere. Incrasing rolation rate also reduces the pressure maximum near the forward stagnation point and displaces it towards the advancing side of the sphere. 


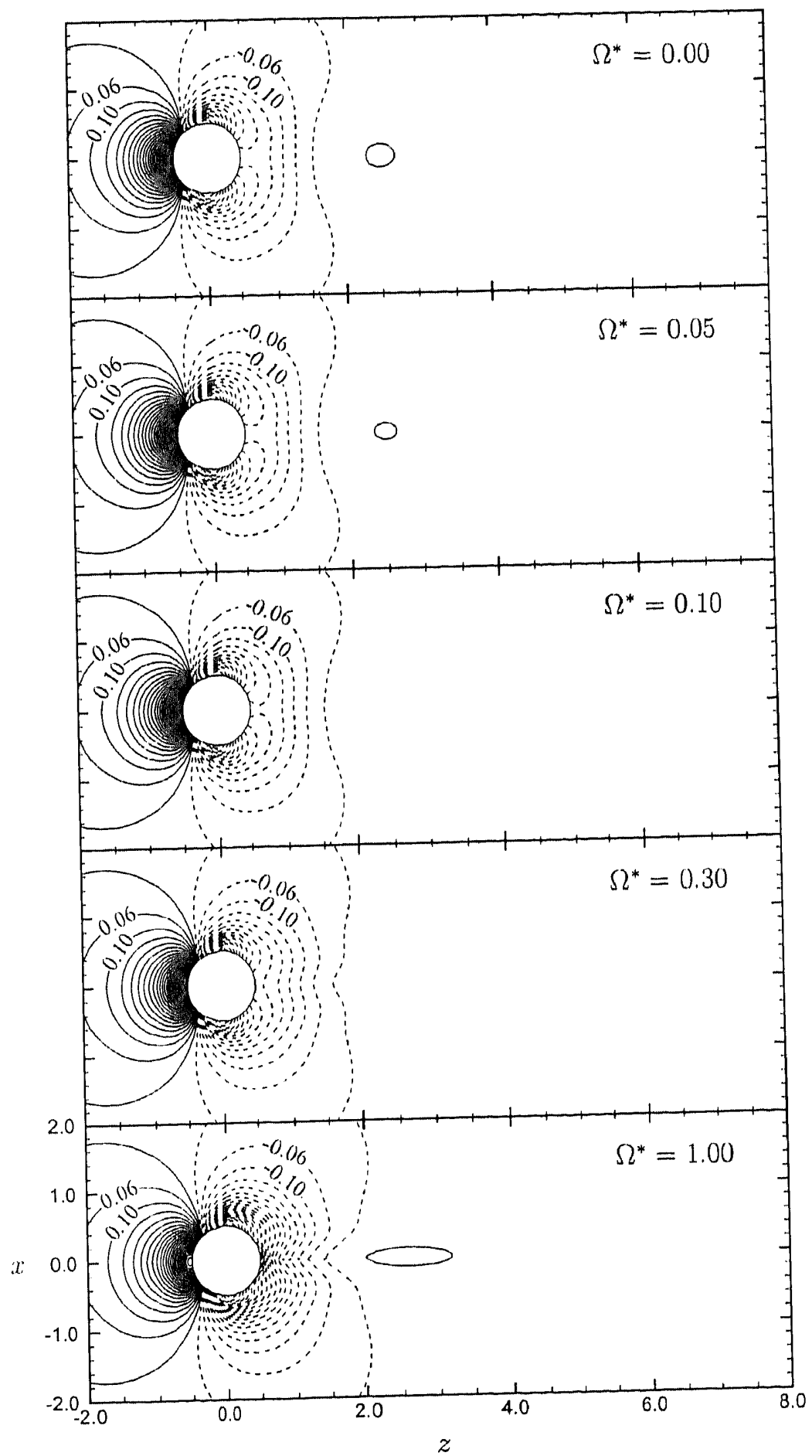

Figure 5.35: Pressure coefficient contours on the $(x, z)$-plane for $R e=100$. 


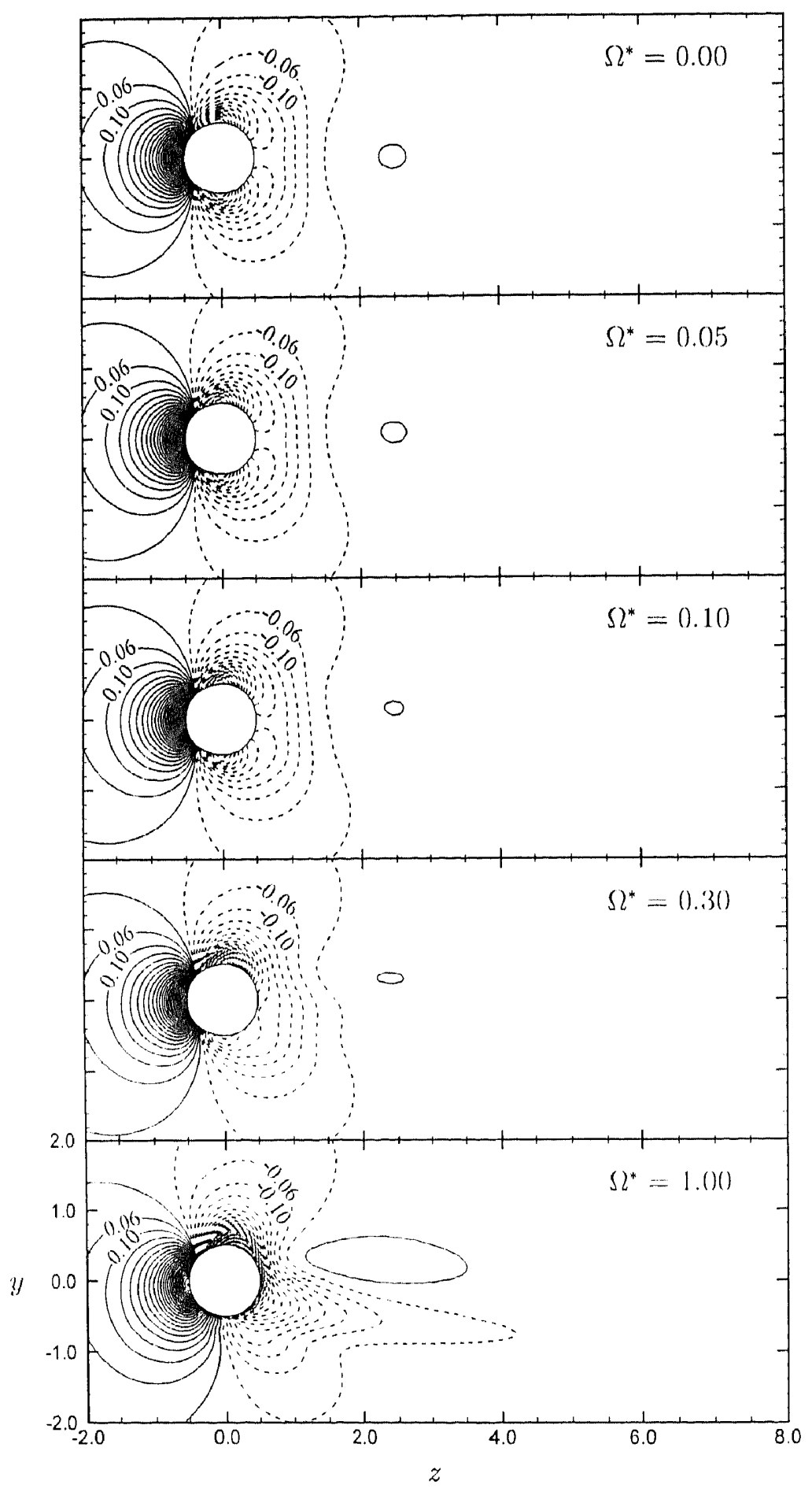

Figure 5.36: Pressure coefficient contours on the $(y, z)$-plane for Ree $=100$. 

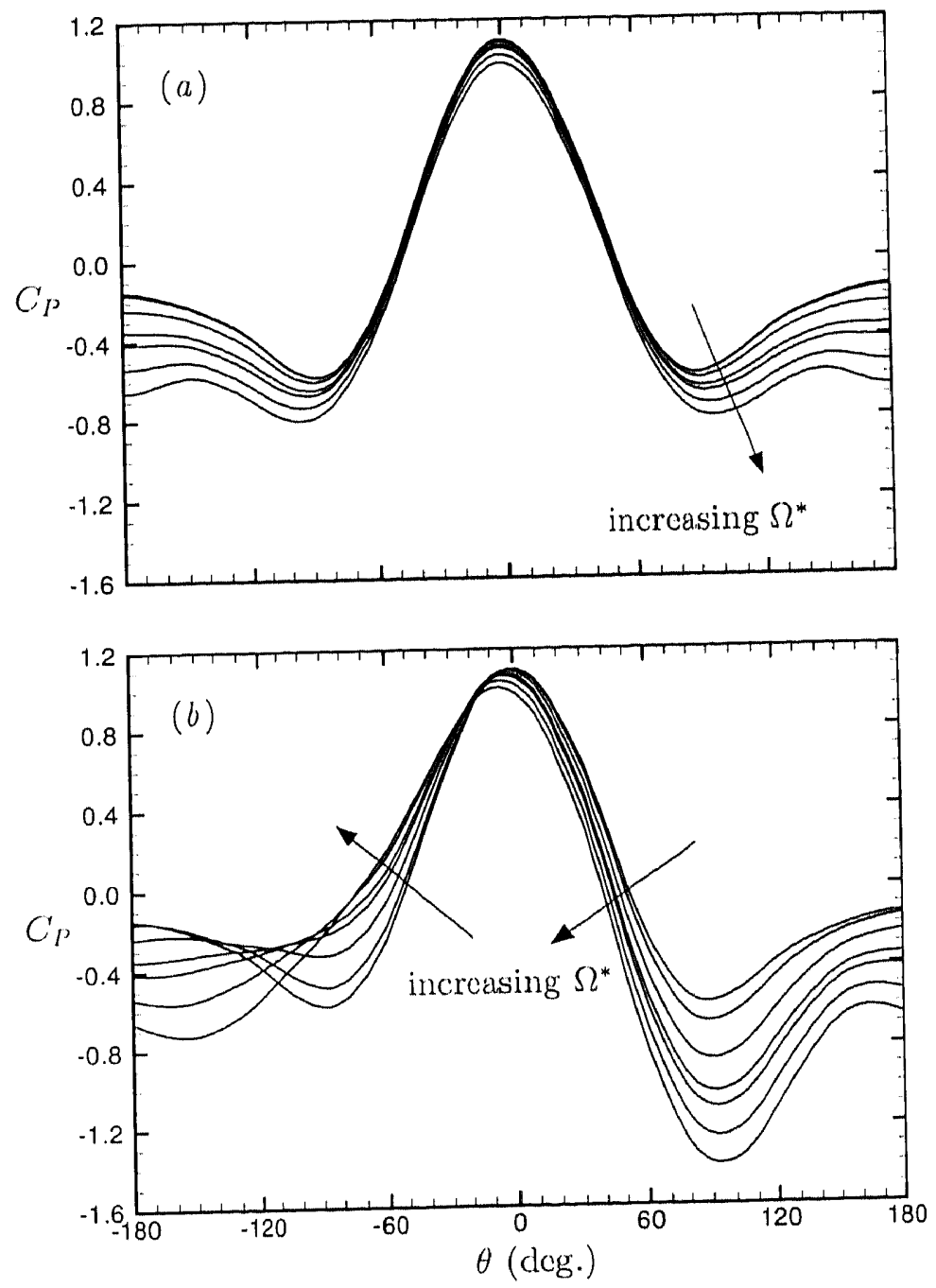

Figure 5.37: Pressure cocfficient, $C_{P}$, along the sphere surface for Re $=100$ and $\Omega^{*}=0.00,0.10,0.30,0.50,0.60,0.80$ and 1.00: $(a)$ Profiles on the $(x, z)$-plane $(\phi=0) ;(b)$ Profiles on the $(y, z)$-plane $(\phi=\pi / 2)$. 
For $R e=250$ the pressure coefficient ficld on the $(x, z)$ - and $(y, z)$-plancs is shown for a number of representative rotation rates in figures 5.38 and 5.39 , respectively. For those cases where the flow is unstcady, the instant shown coincides with the lateral lift force being at a local minimum. For a stationary sphere a pair of closed contours exist on the $(x, z)$-plane. The position of these pressure minima coincide approximately with the center of rotation of the toroidal wake and arise to balance the centrifugal accelcration caused by the vortical motion. On the $(y, z)$-plane the pressure coeflicient ficld is not symmetric, with the pressure over the upper side of the near-wake being lower than that on the lower side. Johnson \& Patcl (1999) noted that an azimuthal pressure gradient exists along the core of the toroidal wake and this drives flow along its axis from the high pressure to low pressure side. The corresponding streamline pattern is presented in figure 5.15 and shows a topologically stable lower focus on the high pressure side, which expels fluid normal to the plane and feeds the unstable upper focus where the lower pressure acts as a sink.

With the introduction of sphere rotation, the pressure minima found on the $(x, z)$ plane become less intense and are drawn towards the lee side surface of the sphere. For $\Omega^{*}=0.10$, they have coalesed with the low pressure lobes on either side of the sphere and for $\Omega^{*}=0.20$ they are absent. At $\Omega \Omega^{*}=0.10$ there is the applarance of a pair of symmetrie protuberances near $z=2.0$ (indicated by arrows). These protuberancess become more pronounced for $\Omega^{*}=0.20$, but diminish at $\Omega^{*}=0.30$ and are alsent at, higher rotation rates. These are the signature of the low pressure streamwise legs of the hairpin vortices which are seen in the vortical wake structures presented in the previous sections. Nlthough the streanwise tails become stronger with increasing $\Omega^{*}$, their effect on the in-plane pressure field is less felt because the legs become increasingly deflected away from the $(x, z)$-plane. At higher rotation rates the pressure field rescmbles that at $R e=100$.

On the $(y, z)$-plane the effect of sphere rotation is to reduce the size of the low pressure lobe on the retreating side of the sphere. This coincides with the reduction 
in size of the upper focus seen in the streamline patterns presented earlicr in figure 5.15. The onset of vortex shedding at $\Omega^{*}=0.10$ is marked by the appearance of a pressure minimum on the advancing side of the sphere near $z=1.00$. For the time instant shown, the corresponding instantaneous streamline pattern is presented in the first pancl of figure $5.17\left(t_{c}^{*}=0\right)$. From the subsequent evolution of the streamline pattern shown in this figure, it is evident that the lower focus attains its maximum size near $t_{c}^{*}=0$, beforc being relcase downstream. The increased strength of the focus combined with its close proximity to the surface leads to a greater suction pressure over the advancing side of the sphere and this results in the lateral lift force being at a local minimum.

For $\Omega^{*}=0.20$, the pressure minimum on the advancing side has grown to form a lobe which is connected to the surface. Under these conditions the oscillatory amplitude of the latcral lift attains a maximum (not shown) and the lateral expansion of the wake is greatest. For $\Omega^{*}=0.30$ the wall-parallel pressure distribution over the lec side of the sphere is noticcably smoother and there is also the appearance of a streamwise tail. This change in the pressure field coincides with the vortical wake structure reverting to a double-thread, while the streamline pattern shows the flow to remain attached over the sphere lee side. At higher rotation rates the wake is steady and the pressure field resembles that for $R e=100$; however, owing to the higher Reynolds number, a lower pressure is attained along the axis of the streamwise tail.

For $R e=300$ the pressure cocfficient field on the $(x, z)$ - and $(y, z)$-planes is shown for a number of representative rotation rates in figures 5.40 and 5.41 , respectively. For a. stationary sphere the pressure field on the $(x, z)$-plane is similar to that for $R e=250$, despite the flow being unsteady. Noticcable differences are the more intense pressure minima on the plane and a pair of symmetric protuberances near $z=2.0$ (indicated by arrows), which at $R e=250$ appear only for $\Omega^{*} \geq 0.10$. With incrcasing rotation rate the pressure minima are shifted towards the sphere surface in a similar fashion to that found for $R e=250$. The protubcrances grow initially with increasing rotation 
rate and become most prominent at $\Omega^{*}=0.10$, before diminishing again. Between $\Omega^{*}=0.00$ and 0.10 , a pair of pressure minimum exist near $z=7.0$. These minima coincide with the locations where the legs of a shed hairpin structure intersect the plane. For $\Omega 2^{*}=0.30$ the pressure minimum appear further upstrcam noar $z=5.0$. At this rotation rate the hairpin structures shed from the sphere assume a curledup S-shape, and, at the time instant presented, the legs of the hairpin oseulate the $(x, z)$-plane rather than ponctrate it.

For $\Omega^{*}=0.50$ to 0.60 the wake reverts to a steady double-threard structure. 'The low' pressure cores of the tails are deflected away from the $(x, z)$-plane and clo not give rise: t.o the low-pressure protuberances that are found at lower rotation rates. leor $\Omega \imath^{*}>0.80$ the onset of a second vortex shedding regime is not accompanied by a notuble change in the near-wake flow and the pressure field resembles that at lower Reynolels number, where the flowfichl romains steady.

On the ( $y, z)$-plane, a pressure minimum ocenes on the advancing side of the sphere now $z=1.00$ for $\Omega^{*}=0.0(0)$ to 0.30 . This minimun coincides with the conter of the stable focms secen in the streamline pattern. With increasing rolation rate the pressure minimum becomes more intense up tos $\Omega^{*}=0.10$. Over the range $\Omega^{+}-0.001$ (1) 0.10

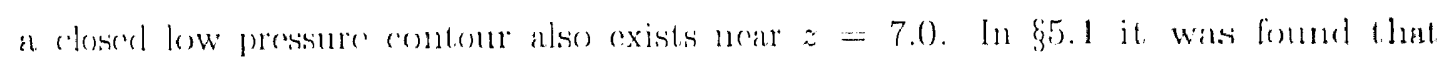
hairpin structures shed from the sphere mederge a reconnection miclway along their

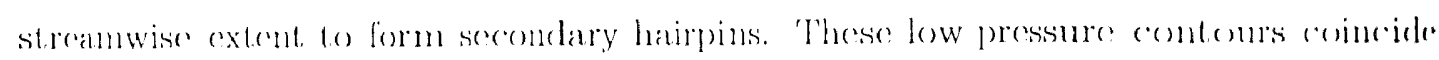
with the position of a secondary hairpin heat. Althongh the primary hairpin intersects the plane noal $z-1.0$ it does not lead to a similar pressure minimum, suggesting that. the rotational strength and resultant centrifugal acceleration induced by the secomdary hairpin is grenter than that of the primary structure.

For $\Omega 2^{*}=0.30$ the hairpin structure shed from the sphere curl-up inte a $\Omega 2$-shape and the secondary hairpin structures are absent. At the time instant shown, a pressure minimum exists near $z=2.5$ which is connected to the noar-wake by a tail. This is the signature of a hairpin structure that is about to be released from the noar-wake. 
The low pressure contours near $z=6.0$ coincide with the location of a hairpin shed at the previous cycle.

For $\Omega^{*} \geq 0.50$ the near-wake wall-parallel pressure ficld is smoother and there is the appearance of a streamwise tail. The absence of low pressure closed contours in the near-wake coincides with the wake reverting to a steady double-thread structure. For $\Omega^{*} \geq 0.80$ the low pressure tail becomes perturbed, while for $\Omega^{*}=1.00$ there is a series of pressure minima anchored along the tail. In figure 5.23, the streamline pattern for $\Omega^{*}=0.80$ was presented in a reference frame moving with the local flow and consists of a scries of stable foci separated by saddles. The pressure minima coincide approximately with the centers of the stable foci, while the pressure maxima, scparating the pressure minima, are centered near the saddle points. The transition to a second vortex shedding regime is not accompanied by a noticcable change in the ncar-wake pressure ficld.

To illustrate the effect of the Reynolds number, figure 5.42 presents time-averaged surface pressure coefficient profiles on the $(x, z)$ - and $(y, z)$-plane for the three Reynolds numbers simulated. For clarity only the rotation rates $\Omega^{*}=0.00,0.50$ and 1.00 are shown. Despite notable differences in the instantancous pressure fields for $R e=250$ and $R e=300$, the time-averaged pressure profiles show very close agrecment. For $R e=100$ the profiles on the $(x, z)$-plane display lower pressure minima and these oceur further from the forward stagnation point than at the higher Reynolds numbers. For a stationary sphere, the pressure field on the $(y, z)$-plane is asymmetric for $R e=250$ and $R e=300$. As a result, the profile at $R e=100$, when compared to the higher Reynolds number cases, shows closer agreement over the retreating side $(\theta<0)$ than over the advancing side $(\theta>0)$. 


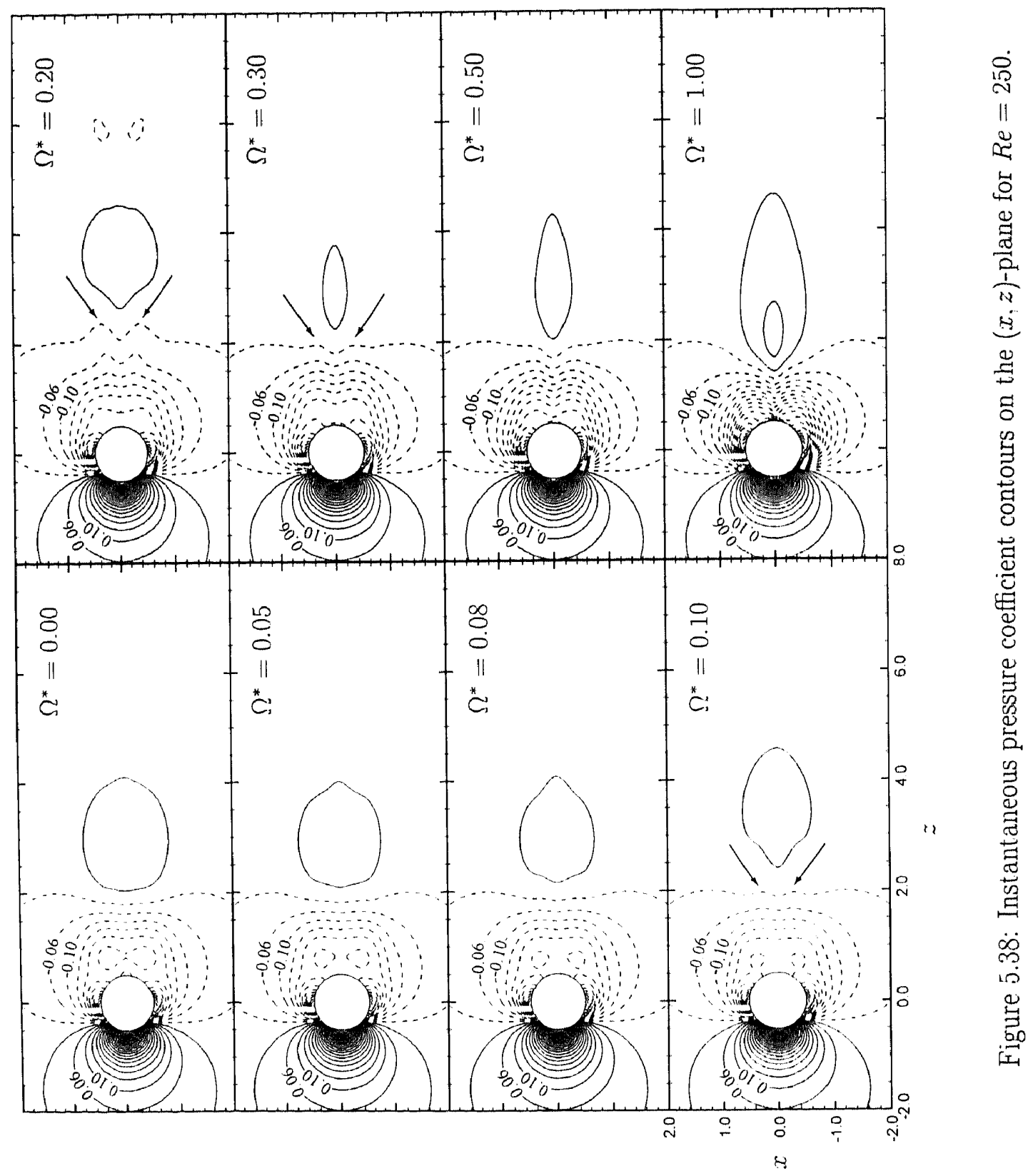




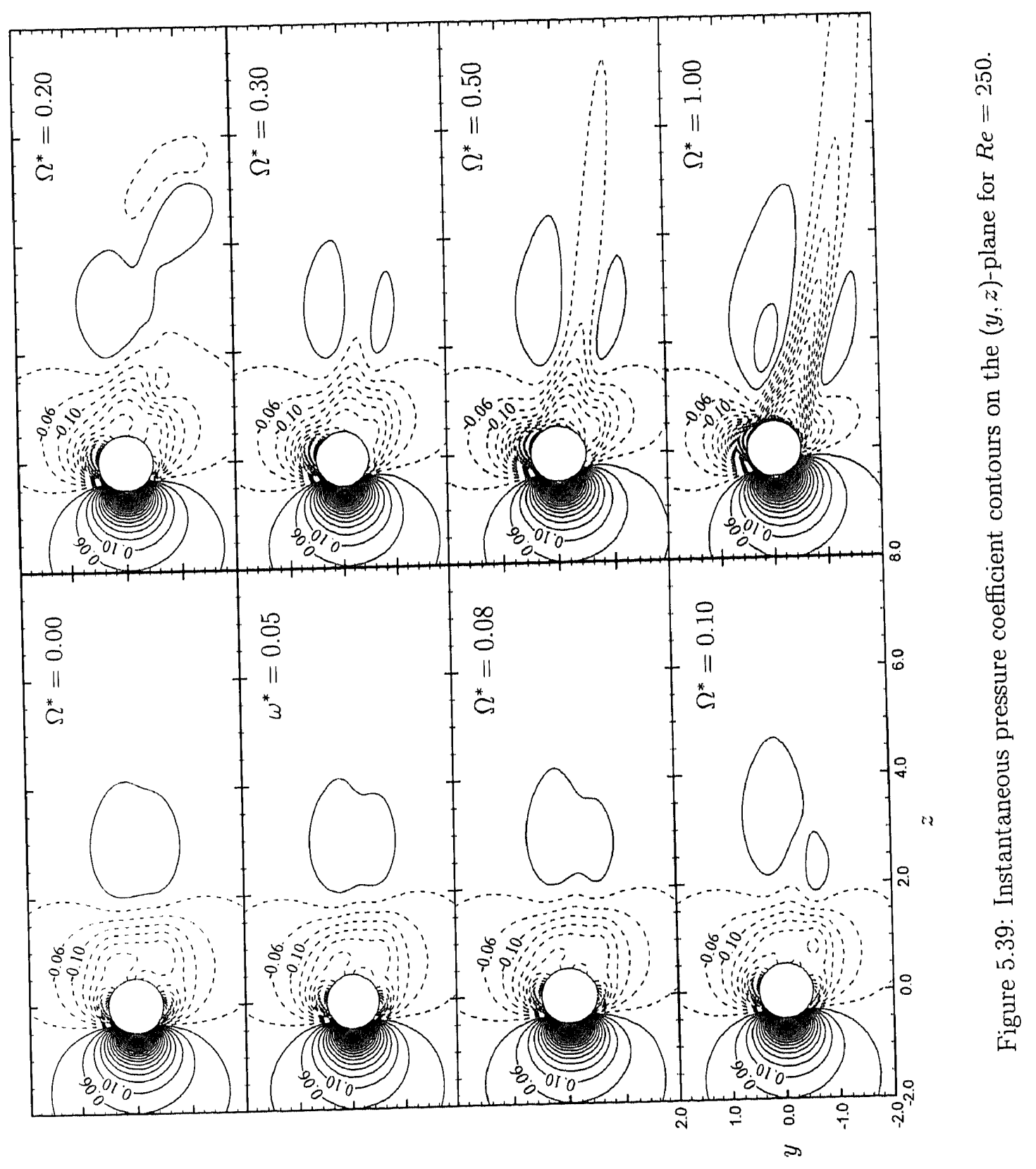




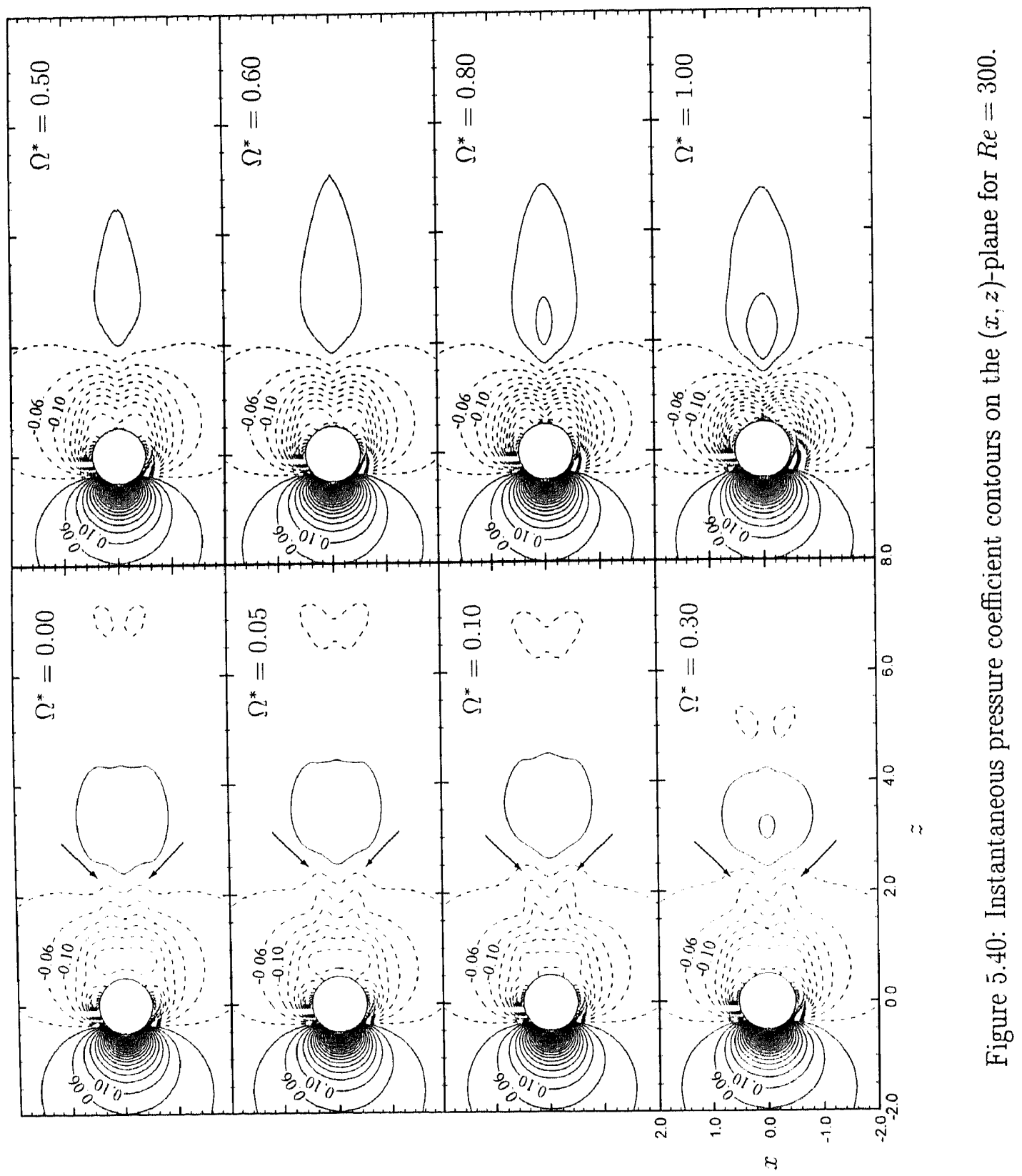




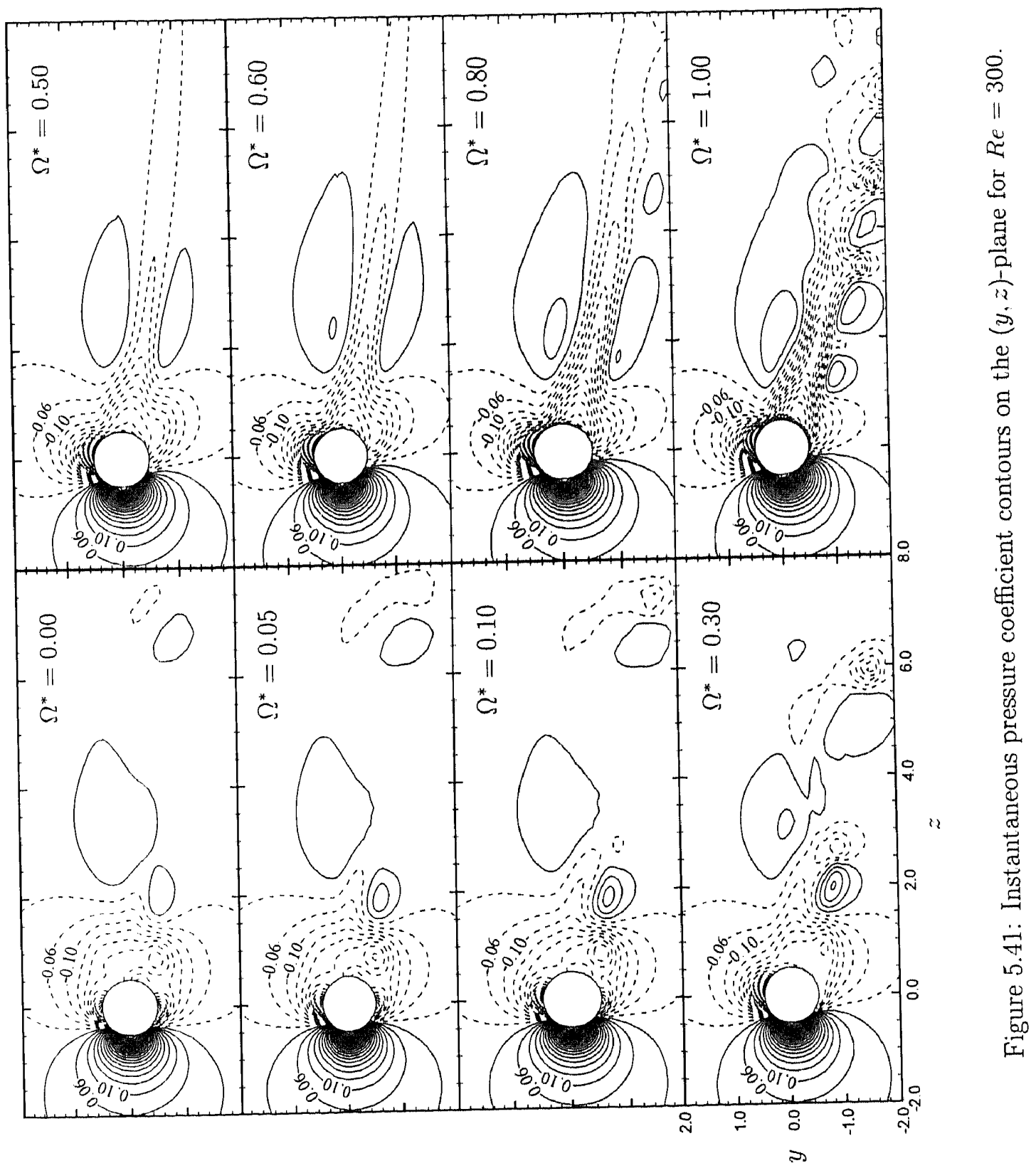



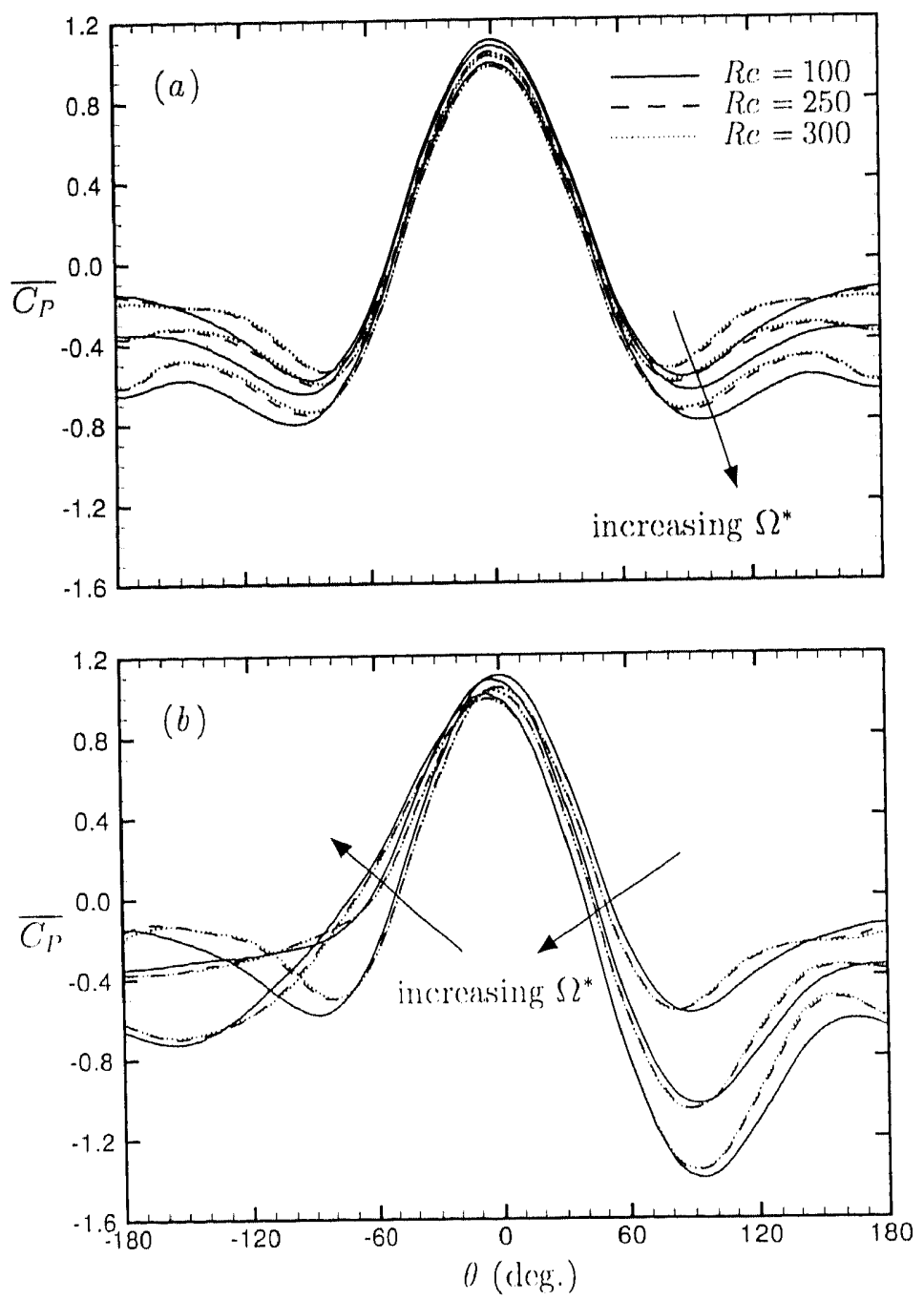

Figure 5.42: Time-averaged pressure coeflicient, $\overline{C_{P}}$, along the spluere surface for $R e=100,250$ and 300 and $\Omega^{*}=0.00,0.50$ and 1.00: (a) Proliles on the $(x, z)$ plane $(\phi=0) ;(b)$ Profiles on the $(y, z)$-plane $(\phi=\pi / 2)$. 


\subsection{Force coefficient histories}

In the modelling of particulate flows, often the intricate nature of the wake is not of interest, but rather the forces and moments experienced by a particle. Time-historics of the drag coefficient, $C_{D}$, and lift coefficient, $C_{L y}$, are presented in figures $5.43,5.44$ and 5.45 for $R e=100,250$ and 300, respectively. Here the lift and drag coefficients are defined as

$$
C_{L y}=\frac{L y}{\frac{1}{2} \rho U_{\infty}^{2} S}
$$

and

$$
C_{D}=\frac{D}{\frac{1}{2} \rho U_{\infty}^{2} S},
$$

where $L y$ is the net force along the $y$-direction, $D$ is the net forec along $z$-direction and $S=\pi d^{2} / 4$ is the sphere projected area. In these and all subsequent figures that present force coefficient data, the force along the $x$-direction is omitted because the flow maintains a symmetry about the $(y, z)$-plane for all cases considered and therefore it is identically zero. The steps taken to verify that the flow symmetry is physical were discussed in $§ 5.1$.

For $R c=100$, increasing $\Omega^{*}$ results in an increasing level of unstcadiness in the transient solution, but the flow settles to a steady-state over the range of $\Omega^{*}$ considered. For $R e=250$, the foree histories indicate that the flow undergoes a number of transitions with increasing $\Omega^{*}$. At zero rotation, the flow reaches a stcady asymmetric statce, as inclicated by a non-zero $C_{L y}$, while above a critical value of $\Omega^{*}$, the flow undergoes a transition to a unsteady regime. For $\Omega^{*}=0.05$ the amplitude of oscillation in the force coefficients undergoes a gradual decay and the flow eventually returns to steady-state. For $\Omega^{*}=0.08$, the decay rate is reduced further, indicating that the wake is close to the bifurcation between the stcady-state and vortex shedding regimes. At higher $\Omega^{*}$ the unstcadiness is sustained and the force historios are characterized by a single dominant frequency. The oscillation amplitudes of $C_{D}$ and $C_{L y}$ attain maximums in the 
vicinity of $\Omega^{*}=0.20$. For $\Omega^{*}>0.30$, the wake unstcadiness is suppressed and the force cocfficients attain constant valucs. Vortex shedding suppression has been observed experimentally by Best (1998) for $R e<300$ and $\Omega^{*}>0.50$, while, in contrast, Niazmand \& Renksizbulut (2003a) reported a "clear periodic wake" at $R e=250$ and $\Omega^{*}=0.50$. The results presented here are in good agreement with those of Best and later in this section, the cause of the discrepancy with the work of Niazmand \& Renksizbulut will be discussed.

For Re $=300$ (sec figure 5.45), the unsteady forces are characterized by a single dominant frequency. With increasing $\Omega^{*}$ the amplitude of oscillation in the forec historics increase and peak at approximatcly $\Omega^{*}=0.10$. For $\Omega^{*}>0.30$, the unstcadiness in the wake is suppressed, in a similar fashion to that at, $R e=250$. Interestingly, at this higher Reynolds number the wake undergoes a further transition to a second vortex shedling regime for $\Omega^{*}>0.60$. Although not clcarly disccrnable, the force coefficient, historics undergo small periodic oscillations for $\Omega^{*}=0.80$ and the amplitucle and frofuency of oscillation increase for $\Omega^{*}=1.00$. Earlier it was found that. in this second unsteady regime the near-wake resembles that at lower rotation rates, where the wake is steady. The lack of activity in the near-wake explains the relatively low amplitude oscillations in the force histories.

For cases that achint an unsteady solution, the forec histories show a progressive increase in the freguency of oscillation with increasing $\Omega^{*}$. Fignter 5.46 prescents the:

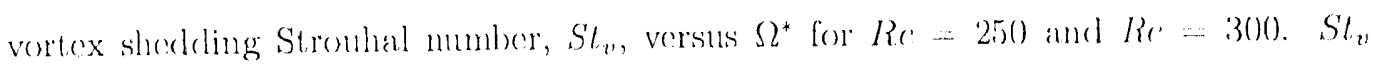
was computed from the power spectrum of the $C_{f}$ and $C_{t, y}$ time-historics and it, was checked by moasuring the period between suecessive peaks in the forec histories. For $R e=250$ and $\Omega^{*}=0.05$ and $\Omega^{*}=0.08$, the amplitude of escillation decays, so $S t_{z}$ is cstimated by directly measuring the period of oscillation from the transiont solution. $S t_{v}$ shows a relatively lincar increase with $\Omega^{*}$, particularly for values of $\Omega *$ below the onset of wake shedding suppression. Although the flow, in the absence of rotation, is significantly different for the two Reynolds numbers, the effect of rotation clrives vortex 

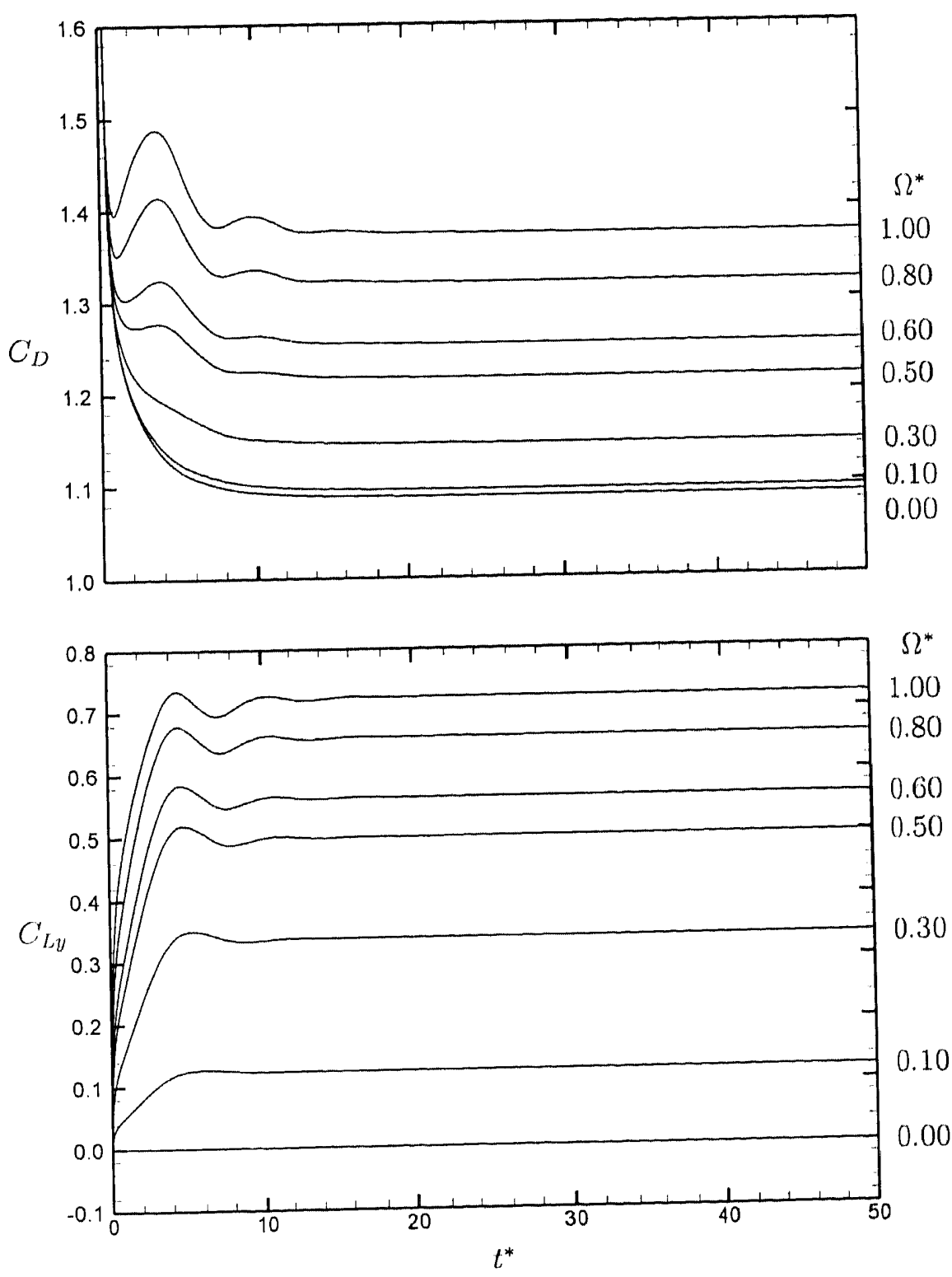

Figure 5.43: Time-histories of the drag coefficient, $C_{D}$, and lift cocfficient, $C_{L y}$, lor $R e=100$ and $\Omega^{*}=0.00,0.10,0.30,0.50,0.60,0.80$ and 1.00 . 

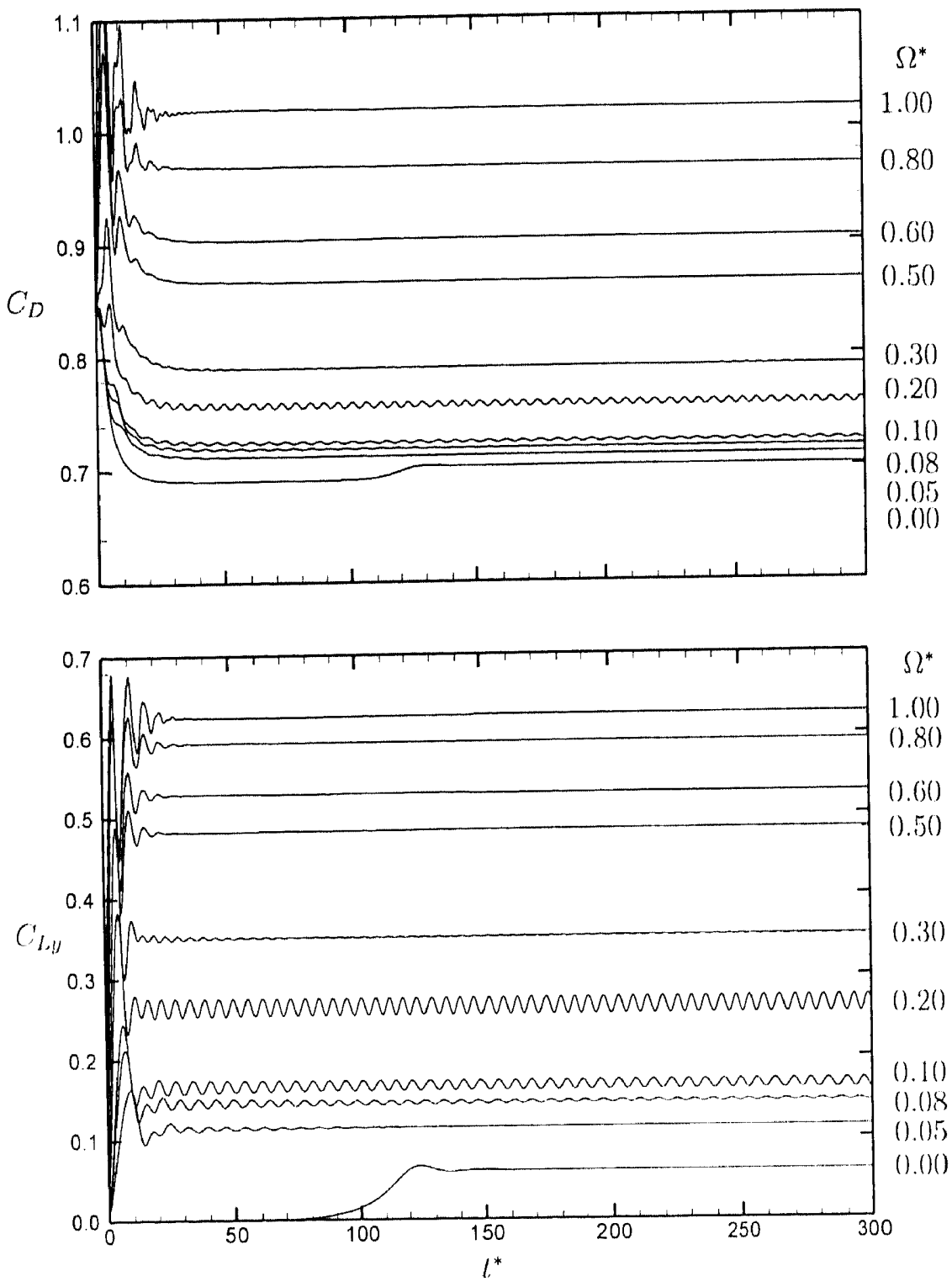

Figure 5.44: Time-histories of the drag coeflicient, $C_{D}$, and lift coeflicient, $C_{L y,}$, for $R e=250$ and $\Omega^{*}=0.00,0.05,0.08,0.10,0.20,0.30,0.5(), 0.60,0.80$ and 1.00 . 

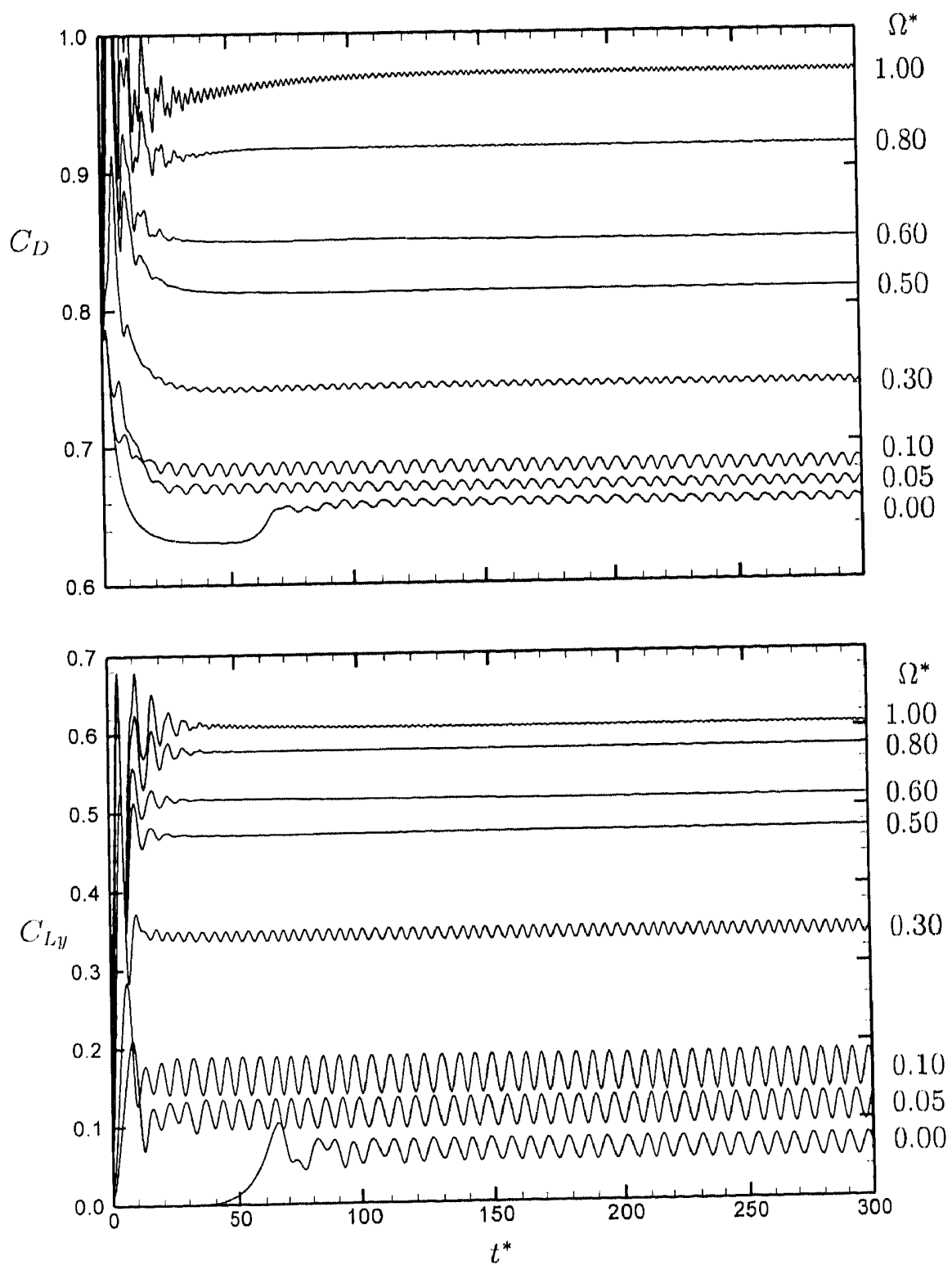

Figure 5.45: Time-historics of the drag cocfficient, $C_{D}$, and lift coefficient, $C_{L y}$, for $R e=300$ and $\Omega^{*}=0.00,0.05,0.10,0.30,0.50,0.60,0.80$ and 1.00 . 


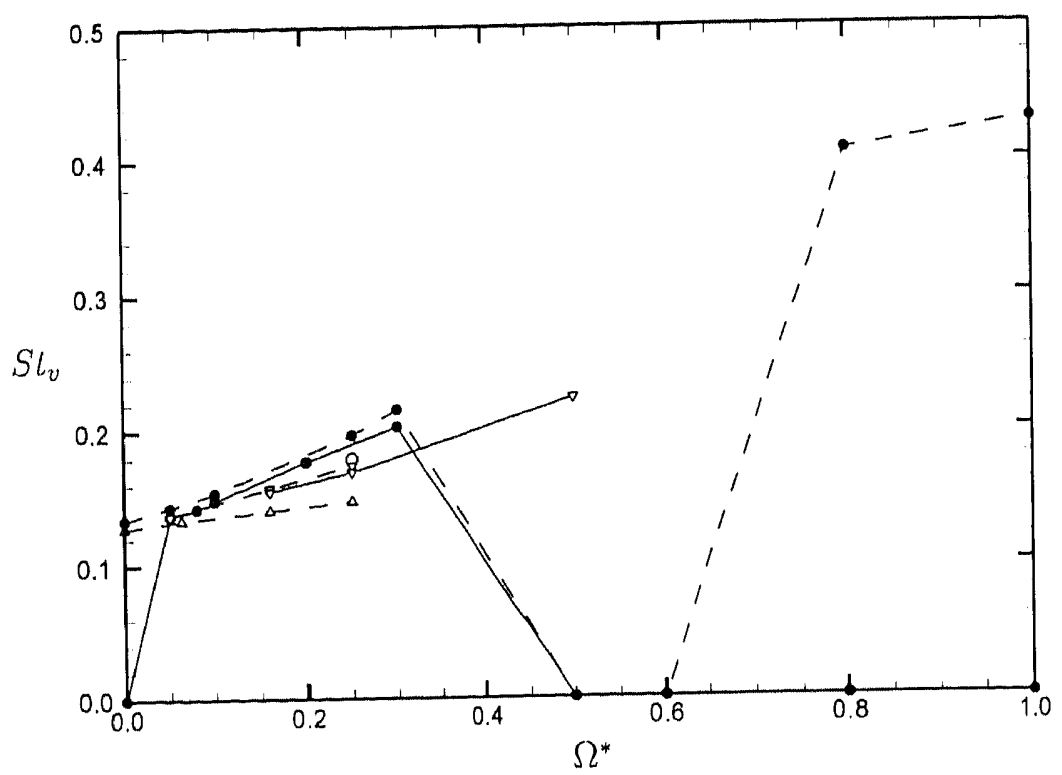

Figure 5.16: Vortex shedding Strouhal number, $S t_{v}$, versus $\Omega^{*}:-\bullet_{-}$, , present study at $R e=250 ;-\bullet-$, present study at $R e=300 ; \circ$, present study at, $R e=300$ using the boundary conditions of Kurose \& Komori (1999); - - - , Niammand \& Renksizbulut, (2003a) at, $R e=250 ;-\nabla-$, Niazmand \& Ronksizhulut, (2003a) at $R e=3(0) ;-\triangle-$, Kurose \& Komori (1999) at $R e=30()$.

shexdeng at, a similar freculency. $\Lambda$ lso shown in this figure are the shedeling frecpuencies computed in the studics of Kurose \& Komori (1999) and Niammand \& Renksizhulut $(2003 \mathrm{a})+\dagger$ Good agreconeme is found only at low $\Omega^{*}$, white the present stondy predicts an increasingly highor shedding frecunency as $\Omega 2^{*}$ increasest.

The time-averaged total, pressure and viscons components of the dragg coeflicient, $\overline{C_{D}}$, and lift coefficiont, $\overline{C_{L y}}$, are presented versus $\Omega^{*}$ in ligures $5.47,5.18$ and 5.49 for Re $=100,250$ and $300 . \ddagger$ Also presented are the todal values computed by Kurose \& Komori (1999) for Re $=100$ and $R e=300$ and by Niazmand \& Renksizbulut (2003a) for $R e=100, R e=250$ and $R e=300$. For all three Reynolds mumbers the?

$\dagger$ Niazmand \& Renksizbulut (2003a) do not present $S t_{v}$ and so the shedeling frecpuency is estimated by measuring the period of oscillation from the lift coefficient histories.

$\$$ For $R e=100$ the reader is reminded that the flowfield remains steady and therefore the time-nveraged force coefficients are equivalent to those computed from the fully developed instantaneous flowfield. 
viscous component of $\overline{C_{D}}$ remains relatively constant with increasing $\Omega^{*}$, while the pressure component sees a gradual increase. With incrcasing Reynolds number, the pressure component comprises a greater percentage of the total drag, while the total drag is reduced. The experimental study of Tsuji et al. (1985) considered $R e=550$ to $R e=1600$ and $\Omega^{*}<0.7$, and in their analysis assumed the drag coefficient not to be influenced by rotation. In light of the present results, this assumption appears to be unsupported. The lift coefficient is composed predominantly of the prossure component and secs a marked increase with increasing $\Omega^{*} . \dagger$

For $R e=250$ and $R e=300$, the drag cocficient computed by Niazmend \& Renksizbulut (2003a) and Kurose \& Komori (1999) show good agrecment with the prescnt study, while for $R e=100$ these studies predict lower values of drag coefficient. Good agrecment for $\overline{C_{L y}}$ is found only in the vicinity of $\Omega^{*}=0.00$. With increasing $\Omega^{*}$, the current study predicts increasingly higher values of the $\overline{C_{L y}}$. The discrepancy is a result of both Kurose \& Komori and Niazmand \& Renksizbulut applying the same, but incorrect, velocity boundary condition at the sphere surface. Figure 5.50 compares the velocity vectors derived from the velocity boundary conditions used in these and the present study. It is immediately evident that the boundary condition used by Kurose \& Komori and Niazmand \& Renksizbulut fails to correctly prescribe the velocity profile at the surface of a spinning sphere. This velocity boundary condition gencrates less circulation for a given $\Omega^{*}$ and cxplains the lower lift force coefficient, drag force coefficiont and Strouhal number predicted by Kurose \& Komori and Niazmand \& Renksizbulut. The lower circulation is also expected to delay vortex suppression to higher $\Omega^{*}$ and explains why Niazmand \& Renksizbulut observed a sustained periodic vortex shedding for $R e=250$ and $\Omega^{*}=0.5$, while Best $(1998)$ and the present study find vortex shedding to be suppressed under these conditions. The boundary conditions appropriate for the coordinate systems used in Kurose \& Komori and Niazmand \& Renksizbulut.

$\dagger$ In contrast, several numerical studies have found that for a two-dimensional cylinder the drag coefficient decreases with increasing rotation rate. It would be interesting to investigate the reason for this difference between the two-dimensional cylinder and the sphere. 
are derived in Appendix $\mathrm{C}$ and are shown to be different to those actually implemented in these studies.

You et al. (2003) alluded to a problem with the computations of Kurose \& Komori (1999). They considered the problem of a spinning sphere for $0.5 \leq R . c \leq 68.4$. For $R e=1.0$ they found the lift cocfficient to be close to the value theoretically derived by Rubinow \& Keller (1961) for Re less than unity, while: Kurese \& Komori (1999) reported a significantly lower value for these conditions. For $R c=68.4$ and $\Omega^{*}=1.0$, You et al. (2003) reported a lift cocficient of 0.79 and a drag coefficiont of 1.73. Good agroement is found with the present study, which finds a lift coefficient of 0.77 and a drag coefliciont of 1.73 (these results are presented graphically in figure 5.51).

The bounclary condition used by Kurose \& Komori (1999) and Niazmand \& Renksizbulut, (2003a) was introduced into the solver used in the present, sturly and tested for $\Omega^{*}=0.25$ and $R e=100$ and 300 . The fore coefficionts computed for these cases are included in figures 5.47 and 5.49, while the vortex sherdeling Stronhal number for Re $=300$ and $\Omega^{*}=0.25$ is inclucled in figure 5.46 . For both Roynolds mumbers good agreement, is found between the forec coefficients derived from these simulations and the values computed by Kurose \& Komori (1999) and Niazmand \& Renksizbulut (2003a). Good agrecment, is also found betwern the Stronhal number derived for $R e=300$ and $\Omega^{*}=0.25$ and the value computed by Niazmand \& Ronksizhulut. It is not clear why the Strouhal number predieted by kurese \& Komeri for these conditions, is considerably lower. These findings further support the argument that the difference between the results reported in these carlier stndies and the prescont work is a result of the different velocity boundary condition applied at the sphere surface. The code cmployed by Niazmand \& Renksizbulut (2003a) wats also used as the hasis for the study of heat transfer from a heated spinning sphere by Niazmand \& Renksizbulut (2003b) and Niazmand \& Renksizbulut (2004). In light of the crror in the surface velocity boundary conditions, the findings of these studies may also be considered as suspect. 

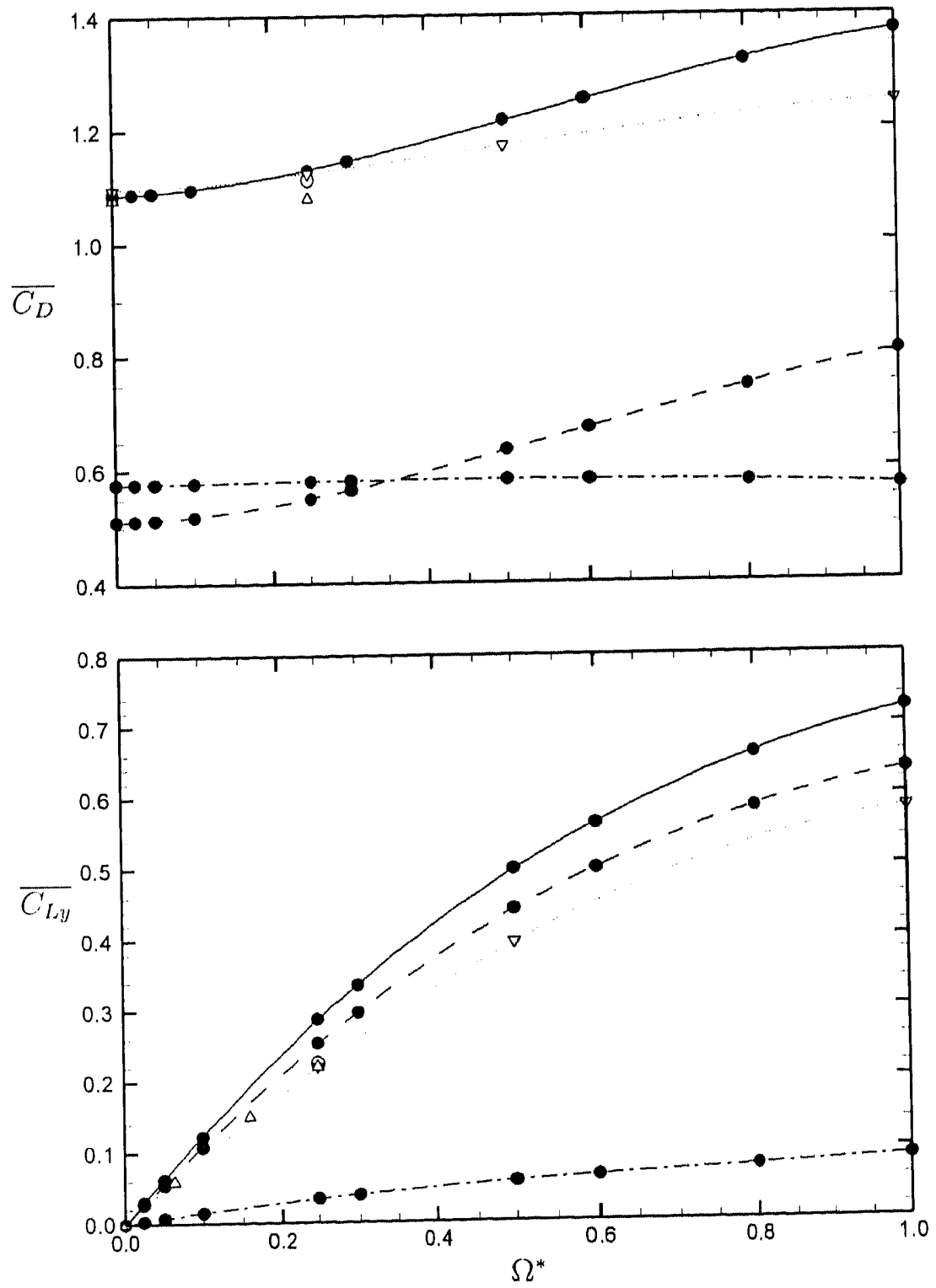

Figure 5.47: Drag cocfficient, $\overline{C_{D}}$, and lift coefficient, $\overline{C_{L y}}$, versus $\Omega^{*}$ for $R e=100$. Present study: -—, total; - - - , pressure component; -....., viscous component. $\triangle$, Kurose \& Komori (1999); …….., Niazmand \& Renksizbulut (2003a); 。 , present study using the boundary conditions of Kurose \& Komori (1999). 

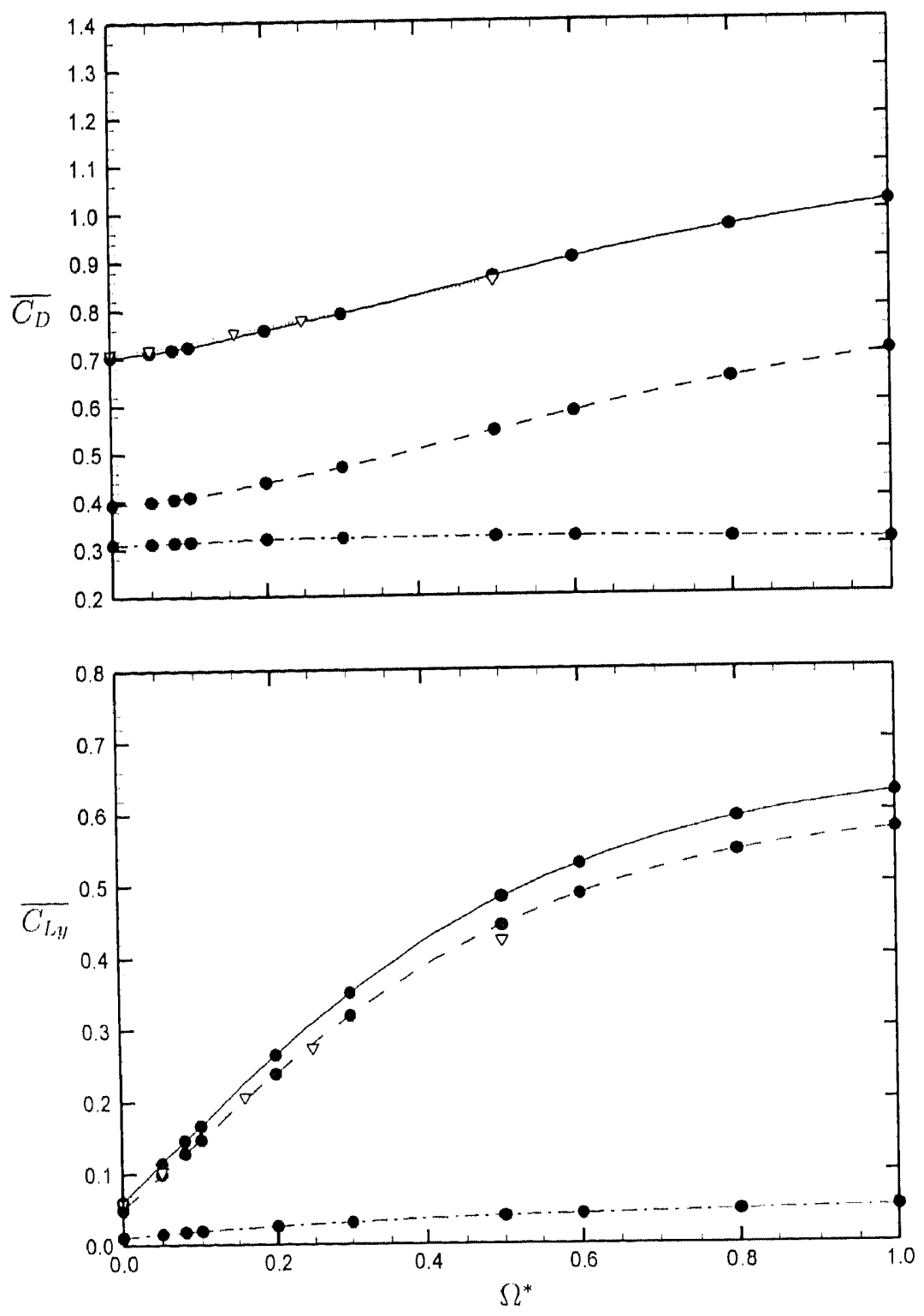

Figure 5.48: Time-averaged drag coefficient, $\overline{C_{D}}$, and lift coeflicient, $\overline{C_{L y y}}$, versus $\Omega^{*}$ for $R c=250$. Present study: $\longrightarrow-$, total; $-\bullet-$, pressire component; $-\cdots \bullet$, viscous component. ……., Niazmand \& Renksizbulut, (2003n). 

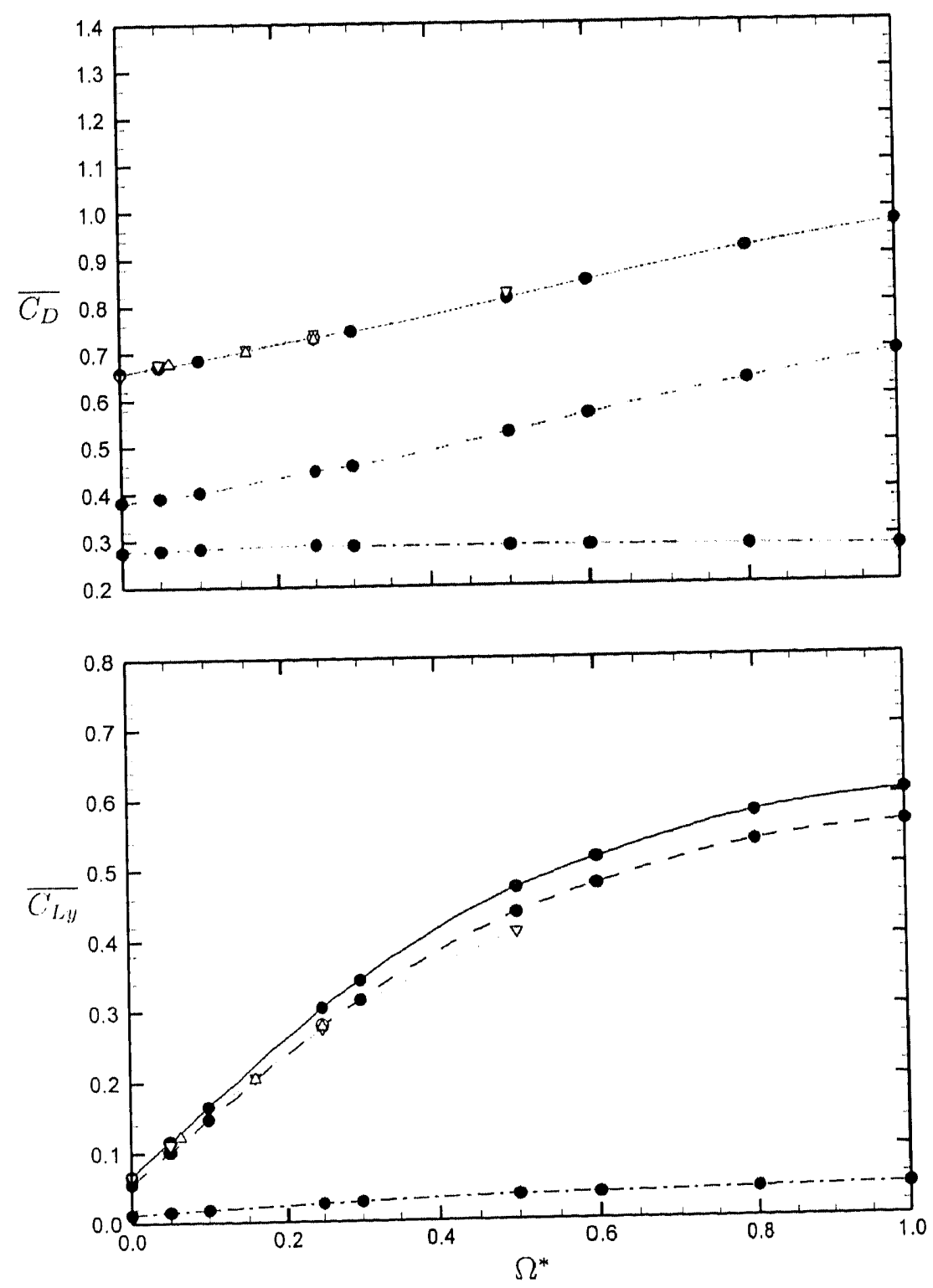

Figure 5.49: Timc-averaged drag cocfficient, $\overline{C_{D}}$, and lift cocficient, $\overline{C_{L y}}$, versus $\Omega \Omega^{*}$ for $R e=300$. Present study: $\longrightarrow$, total; $-\bullet-$, pressure component; $-\cdots \cdots$,

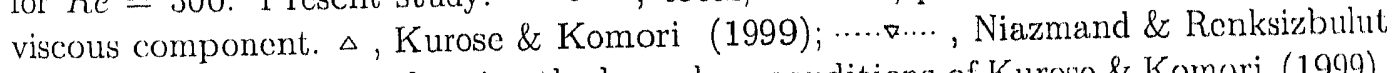
(2003a); o , present study using the boundary conditions of Kurose \& Komori (1999). 

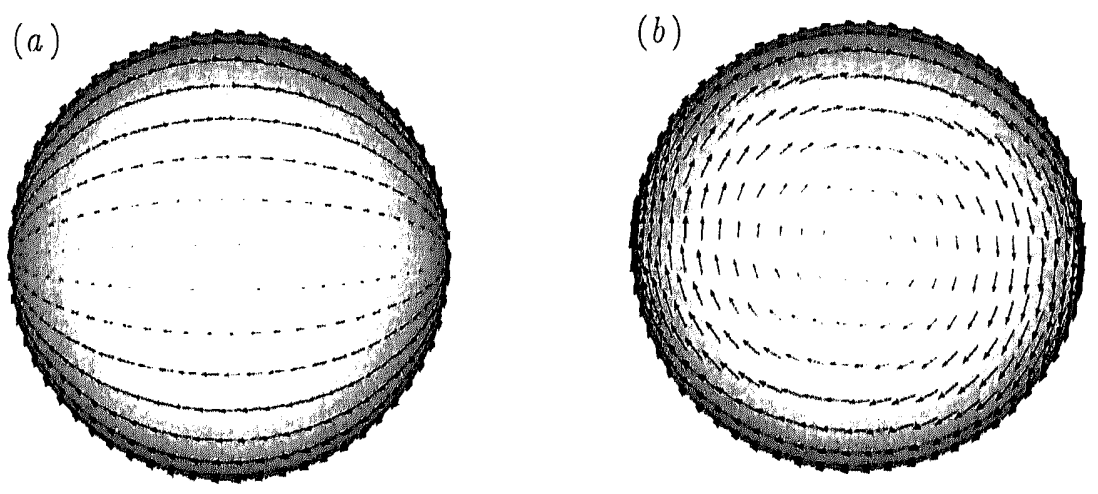

Figure 5.50: Velocity vectors on the surface of the sphere: $(a)$ boundary condition implemented by Kurose \& Komori (1999) and Niazmand \& Renksizbulut. (2003a); (b) boundary condition implemented in the present study. Axis of rotation is normal to the page.

To illustrate the effect of the Reynolds number, figure 5.51 presents the timeaveraged total drag and lift coofficionts versus $\Omega^{*}$. The results for the three Reynolds numbers considered here, together with several carlicr experimental and mumerical results are presented $\dagger$ For low $\Omega^{*}$ the lift coefficiont, shows a small increase with Reynolels number, while at higher $\Omega^{*}$ the opposite trend is eviclent. The results of You ch al. (2003) are consistent with the trends found in the present, study and show close agrecment, for $R e=68.4$ and $\Omega^{*}=1.00$. Despite the experimental uncertainty in the results of Ocsterló \& Dinh (1998), their corrolation for $R r=100$ is in reasonable agrecment, with our results at the same Reynolds number. The correlation of Tsuji ce al. (1985), derived for $R e=500$ to 1600 gives a significantly lower prediction of the lift, coeflecient. The assumption of the lift coefficient, being linearly dependent on the rotation rate is also inconsistent with the behavior found in the other studies.

The drag coefficient decreases with increasing Reynolds mumber for all $\Omega^{*}$ simulated. Due to the difficulties in measuring the drag force experimentally, only the numerical data of You et al. (2003) is available for comparison. The result of You et al. (2003) is consistent with the results of the present study and shows good agrecment for Re $=68.4$

$\dagger$ The results of Kurose \& Komori (1999) and Niazmand \& Renksizbulut, (2003a) are not included for the reasons described earlier in this section. 
and $\Omega^{*}=1.00$. Owing to the limited number of Reynolds numbers considered, the author is cautious not to infer further trends from the data.

Figure 5.52 presents the time-averaged lift to drag ratio, $\overline{C_{L y}} / \overline{C_{D}}$, versus $\Omega^{*}$. Also shown is the analytically derived result of Rubinow \& Keller (1961) for $R e=0.50$. For small Reynolds number, $\overline{C_{L y}} / \overline{C_{D}}$ increases linearly with $\Omega^{*}$, while with increasing Reynolds number $\overline{C_{L y}} / \overline{C_{D}}$ asymptote towards a maximum value. $\overline{C_{L y}} / \overline{C_{D}}$ is a measure of the degree to which the total foree vector becomes tilted away from the streamwise direction and hence with increasing Reynolds number, the total force vector tends towards a fixed orientation. This also suggests that the time-averaged near-wake structure tends towards a fixed orientation with increasing $\Omega^{*}$. Stojković el al. (2002) reported a similar trend for the two-dimensional flow past a spinning cylinder.

Finally, figure 5.53 presents the time-averaged torque coefficient versus $\Omega^{*}$. Here the torque coefficient is defined as

$$
C_{M x}=\frac{M x}{\frac{1}{2} \rho U_{\infty}^{2} S d},
$$

where $M x$ is the net torque about the $x$-axis. The magnitude of $\overline{C_{M x}}$ shows a continual increase with increasing $\Omega^{*}$, while at a fixed $\Omega^{*}$ it decreases with increasing Reynolds number. In contrast, $\overline{C_{L}} / \overline{C_{D}}$ tends to asymptote lowards a maximum value as $\Omega^{*}$ is increased and altains a lower value at lower Reynolds number. This may have ramifications in control applications where sphere rotation is used as a flow control mechanism. Although increasing power (which is proportional to $M x$ ) is recquired to drive the sphere at higher $\Omega^{*}$, this does not lead to a proportional increase in the control aulliority (which may be measured by $\overline{C_{L}} / \overline{C_{D}}$ ). 

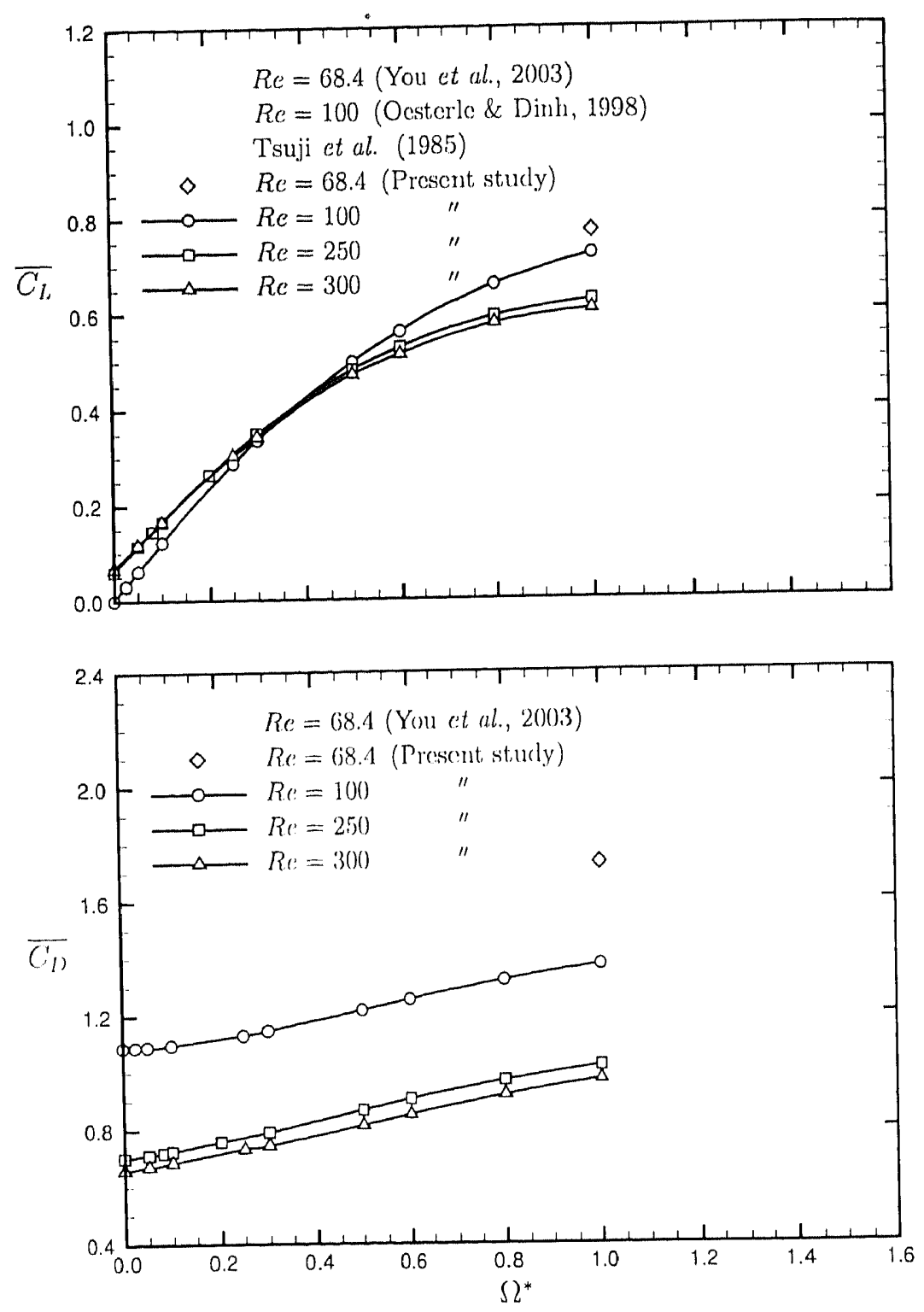

Figure 5.51: Time-averaged total drag and total lift coeflicients versus $\Omega^{*}$ for various Reynolds numbers. 


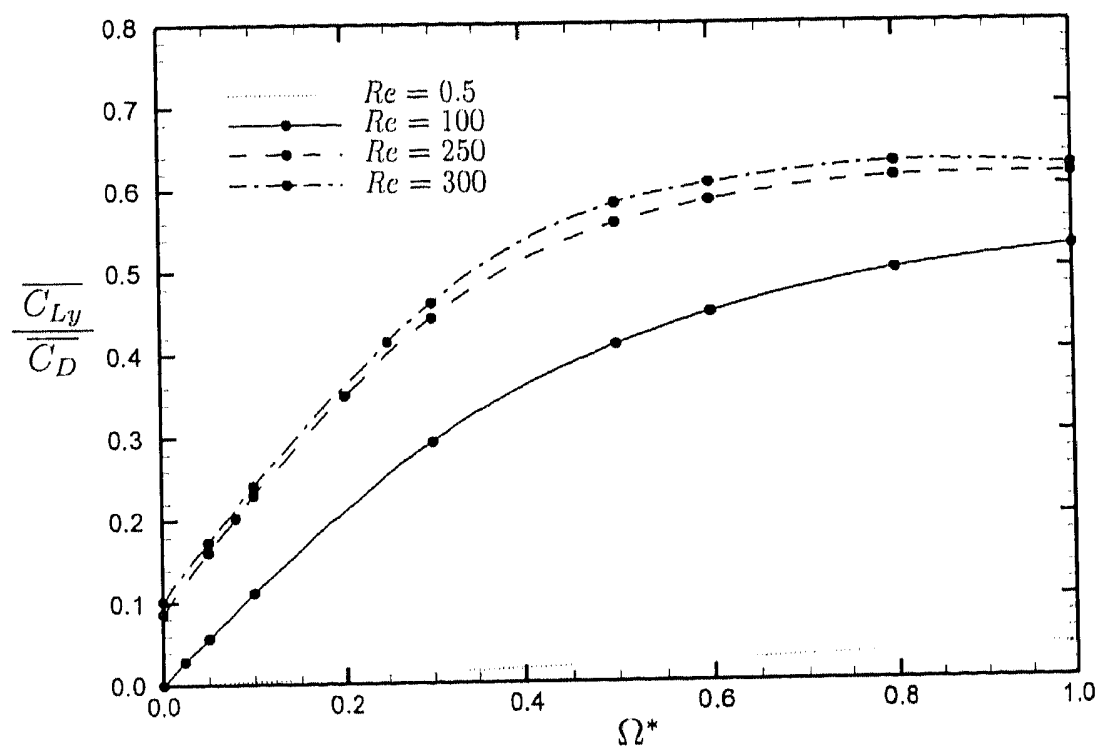

Figure 5.52: Time-averaged total lift to total drag ratio, $\overline{C_{L y}} / \overline{C_{D}}$, versus $\Omega^{*}$.

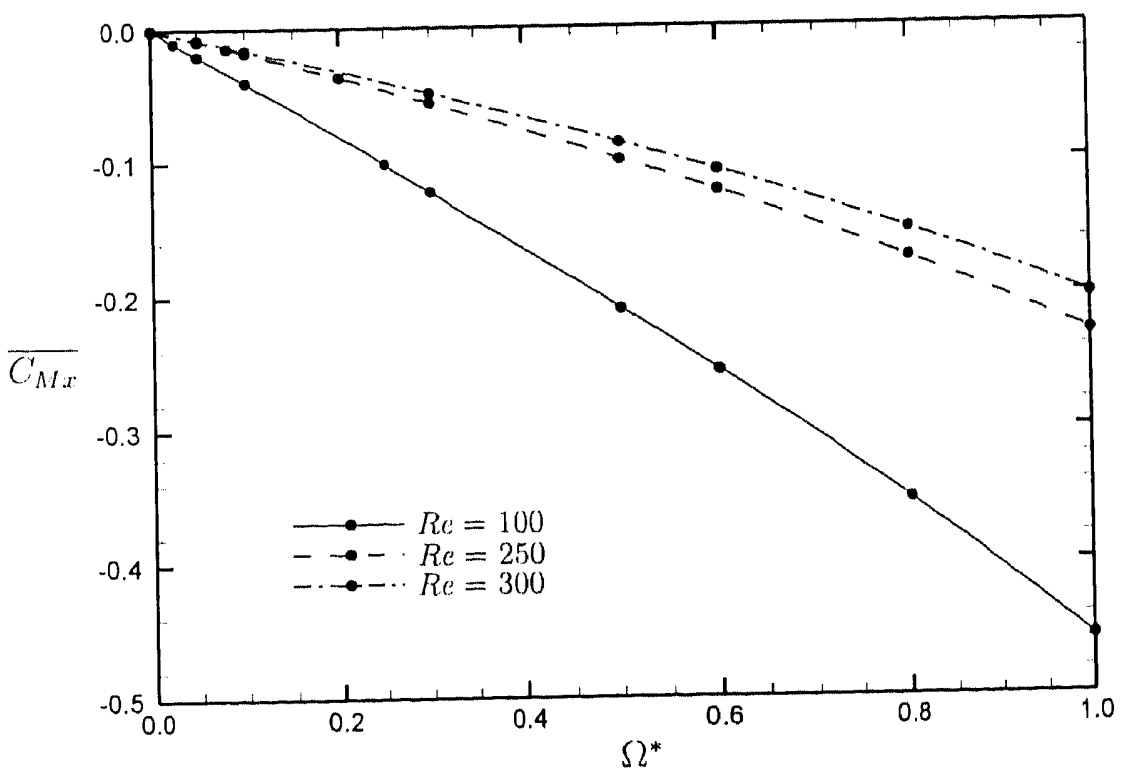

Figurc 5.53: Timc-averaged torque coefficient, $\overline{C_{M x}}$, versus $\Omega^{*}$. 


\subsection{Summary}

This chapter described the evolution of the wake structure behind a transversely rotating sphere for $R e=100,250$ and 300. The main objective was to reach some understanding of the various wake transitions which occur for non-dimensional rotation rates in the range $0.00 \leq \Omega^{*} \leq 1.00$. This was achieved by studying the wake structures as educed using the $\lambda_{2}$ definition of a vortex, the velocity field, vorticity field and pressure field.

For a stationary sphere at $R e=100$ the wake is axisymmetric. Sphere rotation forces a wake transition to a double-thread structure. The cuasi-streamwise threarls comprise streamwise vorticity of equal magnitude and opposite sign. With increasing rotation rate the threads grow in strength and their mutual interaction cansess them to become deflected away from the streamwise axis, in a direction consistent with the classical Robins Magnus effect. Over the range of rotation rates consiclered the wake remains stoady and topologically similar.

For a stationary sphere at, $R e=250$ the wake exists in the asymmetric stoady regime. The introduction of rotation canses a transition to an unsteady wake, which is characterized by the sheeleling of hairpin structures at, a single frecuency. As these structures are convected downstream, there is also the appeatance of so-called induced structures, which have epposite sign and orientation te these redcased from the surfare. The transition occurs in the vicinity of $\Omega^{*}=0.08$. With incrasing rotation rate, frecstream fluid is inereasingly vectored inte the lee side of the sphere and the volume of the recirculating region is progressively reduced until it is completely destreyed near $S \Omega^{*}=0.30$. This transition in the noar-wake topology coincides with the onset of a scond wake transition to a steady double-throad. The resultant structure is similar to that found for $R e=100$. The process of vortex shedeling is often argued to be one of 'build-up and relcase' of vorticity in the near-wake and it, is conjectured that the absence of a recirculation region climinates the conditions necessary for this process to 
occur.

For Re $=300$, the wake of a stationary sphere is characterized by the shedding of like-signed hairpin structures at a single frequency. As these structures are convected downstream, there is also the appearance of induced vortices of opposite sign and orientation. As the rotation rate of the sphere is increased, the shed structures are intensified, while the induced structures are weakened, until the wake undergoes a transition to a stcady double-thread structure in the vicinity of $\Omega^{*}=0.50$. The mechanism driving this transition appears to be identical to that which results in shodding suppression at $R e=250$. In this case the wake undergoes a further transition to a second shedding regime in the vicinity of $\Omega^{*}=0.80$. An examination of the vorticity ficld shows that, with increasing rotation rate, the shear layer separating from the advancing side of the sphere becomes progressively thinner. The evidence strongly suggests that the second transition occurs through a Kelvin Helmholtz instability of this shoar layer. This second transition has, to the authors knowledge, never been observed either through experiment or numerical simulation.

Finally, the force and torque coefficient histories where prosented. It was shown that previous numerical studies into the problem find substantially different forec coefficient and shedding frequencies for the same simulation parameters. The differenee was attributed to these studies using an incorrect velocity boundary condition at the sphere surface. In light of this, it is the authors understanding that the present stucly constitutes the first aceurate numerical investigation of a transversely rotating sphere over the Reynolels number range 100 to 300. 


\section{Chapter 6}

\section{Flow past a sphere undergoing oscillatory transverse rotation}

In chapter 5 steady transverse rotation of a spherical particle was considered. In general, particles in complex flowficlds may undergo unsteady rotation as a result of particle-fluid interactions and collisions with solid boundarics and other particless. Although several studies have considered the problem of a spherical particle in free translation and rotation (sce, for example, Bagchi \& Balachandar (2002a)), the complexity of this problem makes it difficult to establish the physical processes that shape the wake, particularly when the more controlled problem is not yet understood. In this chapter, the transverse sinusoidal oscillation of a spherical particle immersed in a uniform cross-flow is considered.

This secenario also linds application in the control of bluff body wakes, where unsteady forcing has the potential to augment the wake, with the view towards controlling such things as heat and mass transfor, pressure fluctuations, vibration and noise. Understanding the relationship between the unsteady forcing and the wake structure has been the objective of a number of studies dealing with oscillatory forcing of twodimensional cylinders, in which the potential for the effective control of wakes has been demonstrated. The expcrimental study of Tokumaru \& Dimotakis (1991), for example, found that rotary oscillation could lead to a significant drag reduction. Although the flow structure behind a cylinder in rotary oscillation has received considerable at- 
tention in the literature, it is the author's understanding that there are no such studies that have dealt with a spherical body. The objective of this chapter is to describe the wake structure at a single Reynolds number, for a range of forcing frequencies that encompass the natural frequency of vortex shedding. It is hoped that the study will provide some insight into the flow physics and raise further questions to help focus future studies into this problem.

A sinusoidal forcing is prescribed at a frequency, $f_{f}$, and a rotation rate amplitude, $\Omega_{0}$, such that

$$
\Omega(t)=\Omega_{0} \sin \left(2 \pi \int_{f} t^{*}\right)
$$

In this casc the poak angular displacement, $\theta_{0}$ :

$$
\theta_{0}=\frac{\Omega_{0}}{2 \pi \int_{j}}
$$

is an inverse function of the forcing frecuency, while the peak angular acceleration, $\alpha_{0}$ :

$$
\alpha_{0}=2 \pi \int_{\rho} \Omega_{0}
$$

is a lincar function of the forcing frequency. A less commonly empleyed simusoidal forcing is one where the poak angular clisplacenent and forcing frefuency are preseribed as the combrol paraneters. In this case, the angular velecity and angular acceleration take a linear and quadratic degendence on the forcing frecpuency, respectively.

In this stucly, the case of $R e=300$ and a non-dimensional rotation rate amplitude of $\Omega_{0}^{*}=0.50$ was considered. The forcing frecpuneies testerl correspond to

$$
\left.\frac{S t_{f}}{S t_{n}}=0.25,0.50,1.00,1.50,2.00,2.50 \text { and } 3.00\right)
$$

where $S t$, is the Strombal number of forcing, and the Stroulat number of unfored vortex shedding was reported in chapter 3 as $S t_{n}=0.134$ for Re $=300$. For cach simulation, insensitivity to spatial and temporal discretization were verificel. Examples of these checks were presented in chapter 3. The final parameters used for the simulations are summarized in table 6.1 . 


\begin{tabular}{ccccccccc}
\hline$N_{r}$ & $C_{0}$ & $x_{\min }$ & $N_{\theta}$ & $\Theta$ & $N_{\phi}$ & $\Delta t^{*}$ & $d$ & $D_{\mathrm{o}}$ \\
\hline 121 & -0.6 & 0.3 & 100 & -0.35 & 64 & 0.0005 & 1.0 & 22.5 \\
\hline
\end{tabular}

Table 6.1: Spatial and temporal simulation parameters.

The remainder of this chapter is organized as follows: In $\$ 6.1$ the relationship between the forcing and the resultant forees and moments experienced by the sphere are presented. $\S 6.2$ presents the instantancous and time-averaged wake structure. Finally, the important finclings of the chapter are summarized in $\$ 6.3$.

\subsection{Force coefficient histories}

Figure 6.1 presents the time-histories of the total drag coefficient, $C_{D}$, and lift coefficient, $C_{L y}$, for the various forcing frequency ratios, $S t_{f} / S t_{n}$, considered. The sphere angular velocity profile, $\Omega(t)$, is also shown, along which a serics of dots indicating the time instants for which the instantancous wake structure will be presented in $\$ 6.2$. For a stationary sphere, the wake is characterized by the one-sided shedding of hairpin structures and the lift and drag force histories oscillate at the same frecuency. In contrast, oscillatory forcing causes the wake to undergo a two-sided shedding and the drag oscillates at twice the frequency of the lift coefficiont, much like the Kármán vortex street generated downstream of a two-dimensional cylinder at moderate Reynolds numbers. The frecuency and phase relationship between the lift and drag in the statistically steady regime is illustrated by the Lissajous curves presented in figure 6.2. In all cases a closed curve is formed, which is evidence of a periodic vortex formation process. For $S l_{f} / S t_{n}>0$, the Lissajous figures are symmetric about the $C_{L_{\nu}}=0$ line, which indicates that the time-average lift foree is zero when the sphere undergoes forced oscillatory rotation. The curves also serve to highlight the large fluctuating forces that oscillatory rotation can return at certain values of $S t_{f} / S t_{n}$.

The dominant Strouhal number, derived from the power spectra of lift coefficient, history, is plotted against the forcing Strouhal number in figure 6.3. It is cvident that the dominant Strouhal number is synchronized with the forcing frequency. In general, 
a synchronization of the forcing frequency and lift force fluctuations is referred to in the litcrature as "lock-on." The phase angle between the surface velocity and lift coefficient signal, $\Delta \psi$, is presented in figure 6.4. $\Delta \psi=\psi_{f}-\psi_{v}$, where $\psi_{f}$ and $\psi_{v}$ are the phase angles of the prescribed angular velocity and the dominant mode in the lift coefficient time-history. Despite the wake being in a lock-on state, the phase of lift coefficient profile with respect to the body motion varies considerably with forcing frequency. A significant phase shift has also been recorded in the experiments of Ongoren \& Rockwell (1988), where forcing in the form of oscillations in a direction transverse to the incident flow was applied to two-dimensional cylinders of various cross-section. This phase shift is indicative of a shift in the vortex formation phase. At low $S t_{f} / S t_{n}$ the forcing leads the wake shedding by a small phase difference. With increased foreing frequency, $\Delta \psi$ grows such that for $S t_{f} / S t_{n}=1.00$ the peak surface velocity and peak lift coefficient are approximately $90^{\circ}$ out of phase, such that, the lift cocficient attains a local maximum when the angular velocity is in the proximity of zero. $\Delta \psi$ reaches a. maximum in the vicinity of $S t_{f} / S t_{n}=2.00$, before decreasing again at higher $S t_{f} / S t_{n}$. $\Lambda t S l_{f} / S l_{n}=2.00$ the sphere angular velocity learls the lift foree by approximately $140^{\circ}$, such that the lift approaches a local maximum when the angular velocity is at a local minimum. For $S t_{f} / S t_{n}<1.00$, a local maximum in angular velocity corresponds to a positive lift coefficient, which is consistent with that predicted by the classical Robins Magnus offect. For St $/ S t_{n}>1.00$, a local maximum in the angular volocily coincides with a negative lift forec.

The mean drag and the poak-to-peak fluctuating compononts of the drag, lift and torque are shown in figure 6.5. The oscillatory forcing canses the time-alveraged wake to assume a symmetry pattern about the $(x, z)$-plane and as a result the time-averaged lift, force and torque are identically zero. It is evident, particularly for $C_{D_{p} p}$, that, the peak-to-peak viscous and peak-to-pcak pressure force amplitude do not equal the peak-to-peak total force amplitude. This is a result of a phase difference between the viscous and pressure force time-historics. With increasing $S t_{f} / S t_{n}$ the mean drag 
increases towards a maximum near $S t_{f} / S t_{n}=1.00$ bcfore decreasing again. The numcrical simulations of Cheng et al. (2001a) and Cheng et al. (2001b) showed similar behavior for the mean drag experienced by a two-dimensional cylinder undergoing rotary oscillations. Both the fluctuating components of drag and lift, however, are observed to rise sharply towards a maximum near $S t_{f} / S t_{n}=0.50$, before tending towards zero with increasing $S t_{f} / S t_{n}$. The reason for the mean and fluctuating force components peaking at different $S t_{f} / S t_{n}$ is discussed in $\$ 6.2$. Unlike the drag and lift, the torque fluctuations show a continual incrcase with increasing $S t_{f} / S t_{n}$. In the context of wake control applications, though significant authority can be excrcised over unsteady forces experienced by the sphere, this comes at the expense of an increased torque and hence power input. 

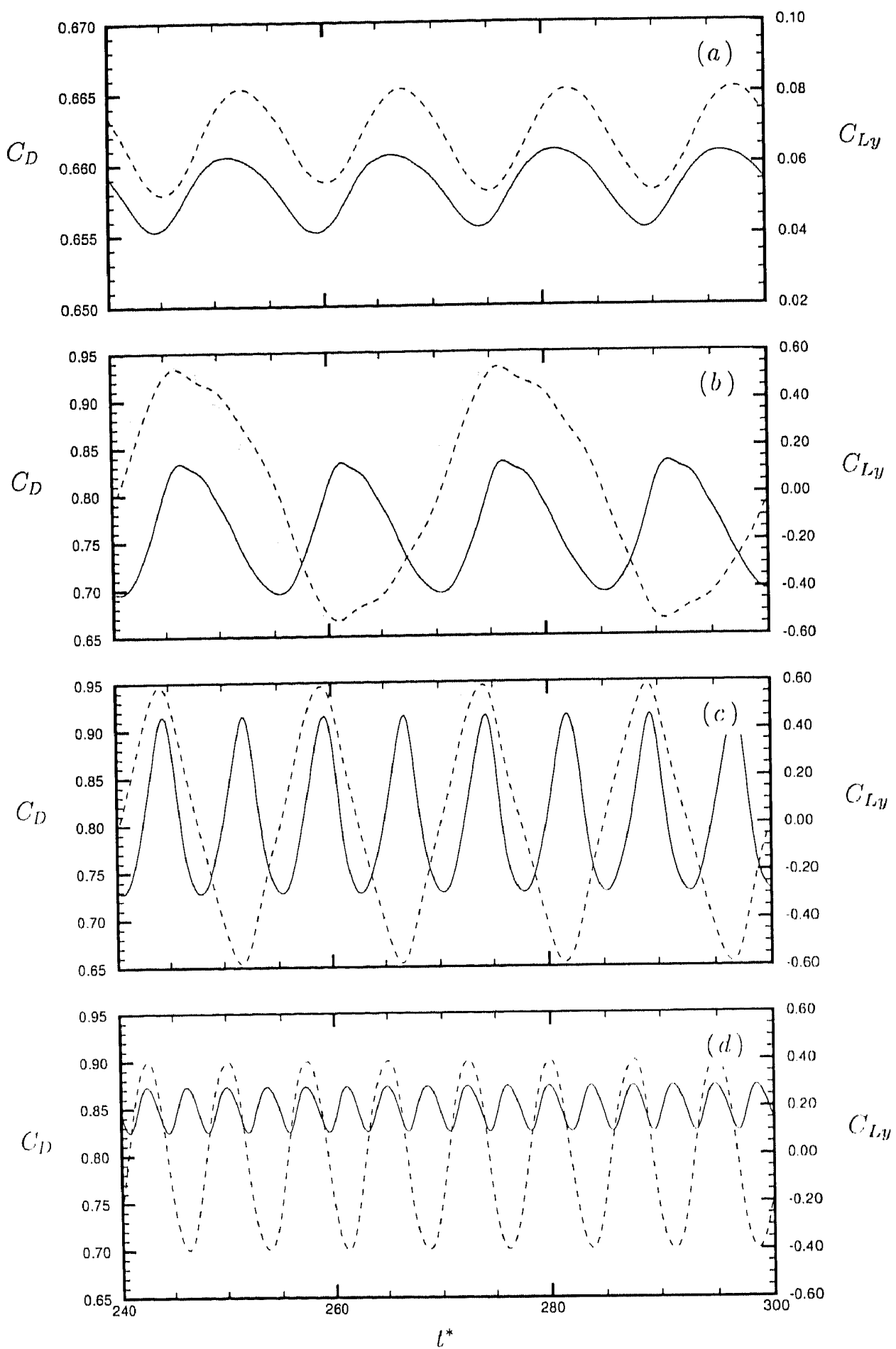

Figure $6.1(a d)$. For caption sec opposite. 

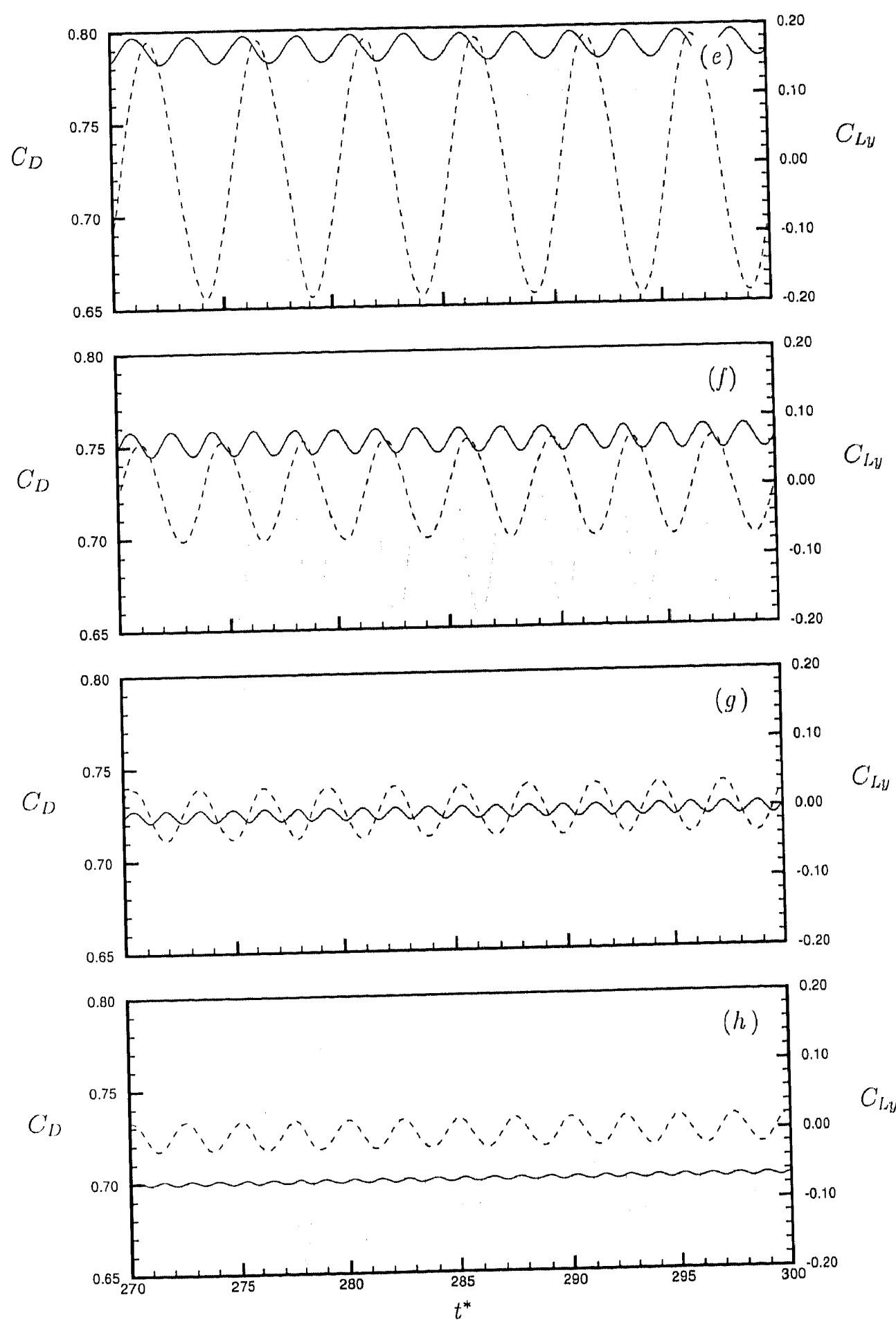

Figure 6.1: Timc-history of the total lift cocfficient, $C_{L y}$, and drag coefficiont, $C_{D}$, and sphere angular velocity for: (a) $S t_{f} / S t_{n}=0.00,(b) S t_{f} / S t_{n}=0.25,(c) S t_{f} / S t_{n}=$ $0.50,(d) S t_{f} / S t_{n}=1.00,(e) S t_{f} / S t_{n}=1.50,(f) S t_{f} / S t_{n}=2.00,(g) S t_{f} / S t_{n}=2.50$ and $(h) S t_{f} / S t_{n}=3.00$.,$C_{L y} ; \cdots, C_{D} ; \cdots, \Omega(t)$. 


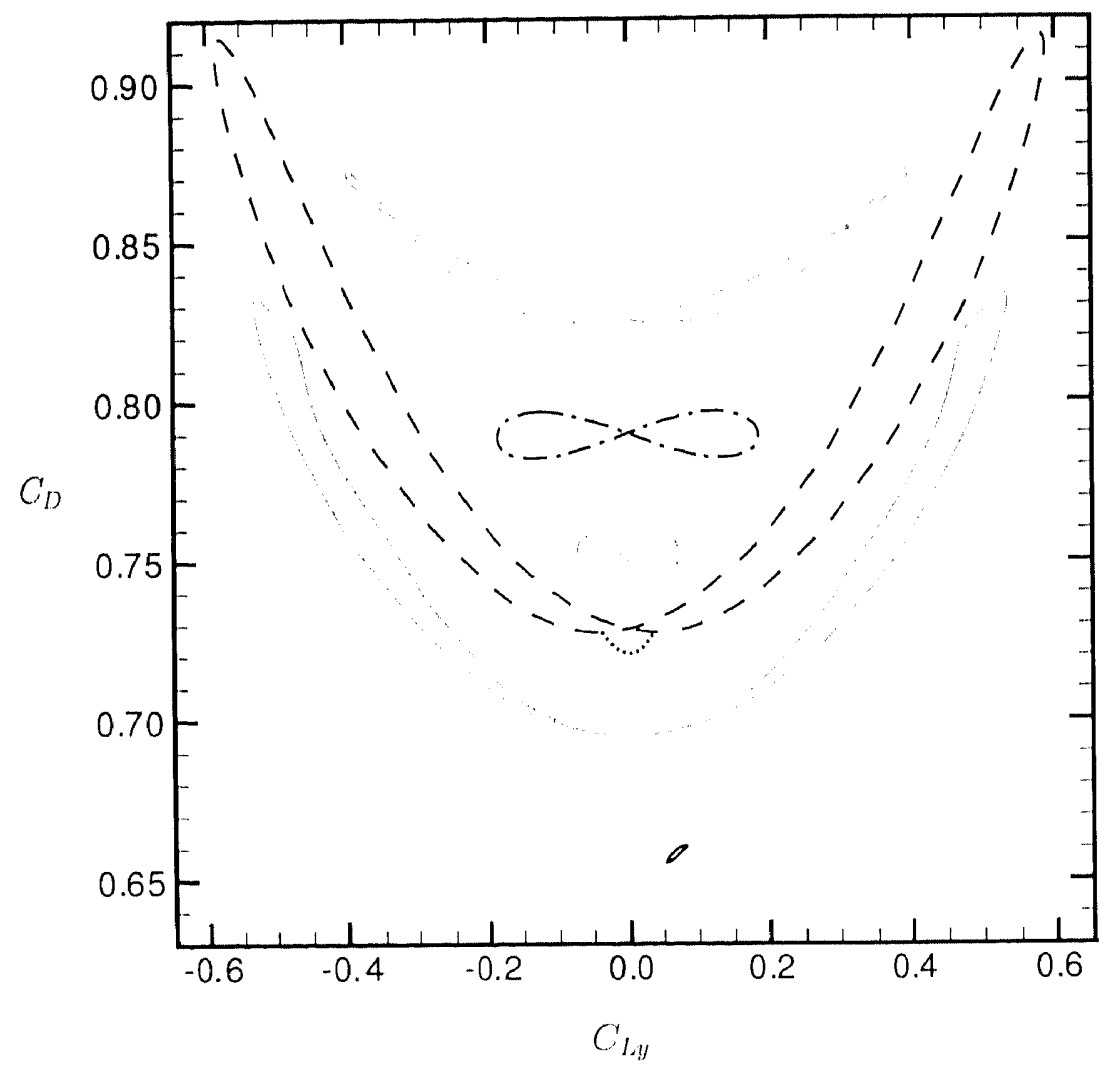

Figure (6.2: Phase diagram of $C_{D}$ versus $C_{L y}$ for various $S t_{f} / S t_{n}$ :

$$
\begin{aligned}
& \longrightarrow, S t_{f} / S t_{n}=0.00 ; \quad, S t_{f} / S t_{n}=0.25 ;--S t_{f} / S t_{n}=0.50 ; \\
& \text {, } S t_{f} / S t_{n}=1.00 ;-\cdot-\cdot S t_{f} / S t_{n}=1.50 ; \quad, S t_{f} / S t_{n}=2.00 \text {; } \\
& \text {, } S l_{f} / S l_{n}=2.50 ; \quad, S l_{f} / S l_{n}=3.00 \text {. }
\end{aligned}
$$




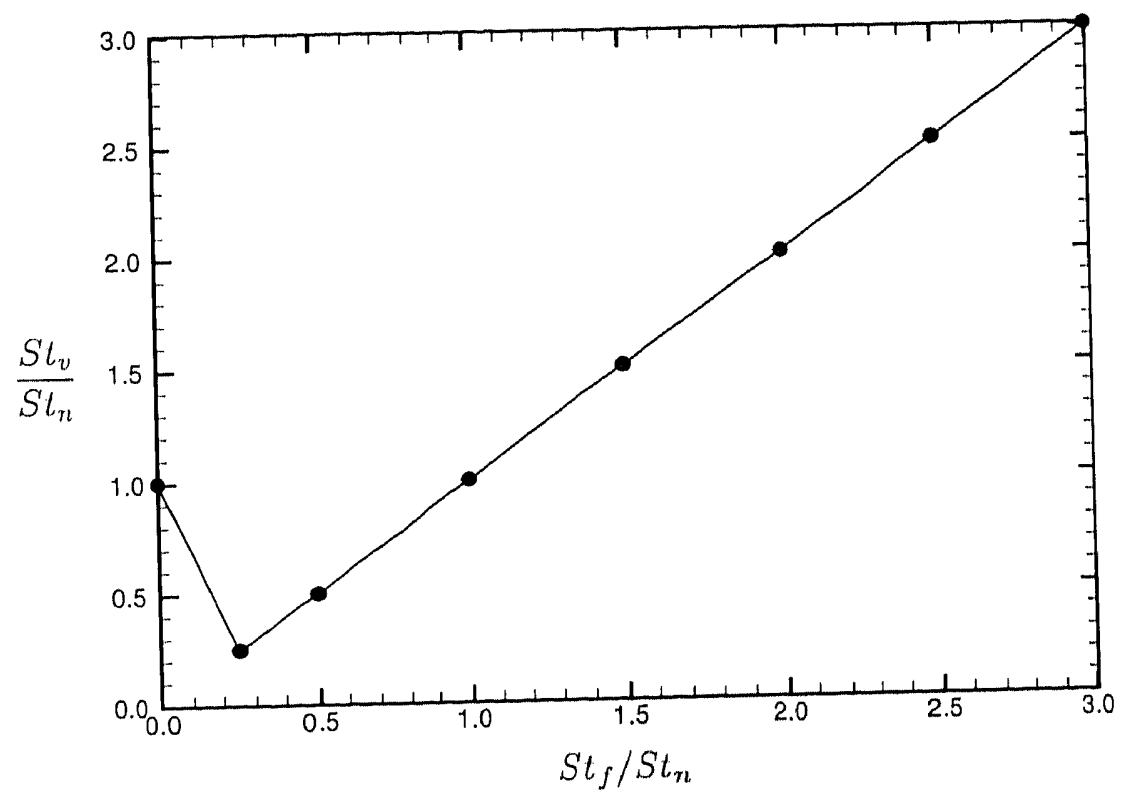

Figure 6.3: Vortex shedding frequency ratio, $S t_{v} / S t_{n}$, versus the forcing frequency ratio, $S t_{f} / S t_{n}$.

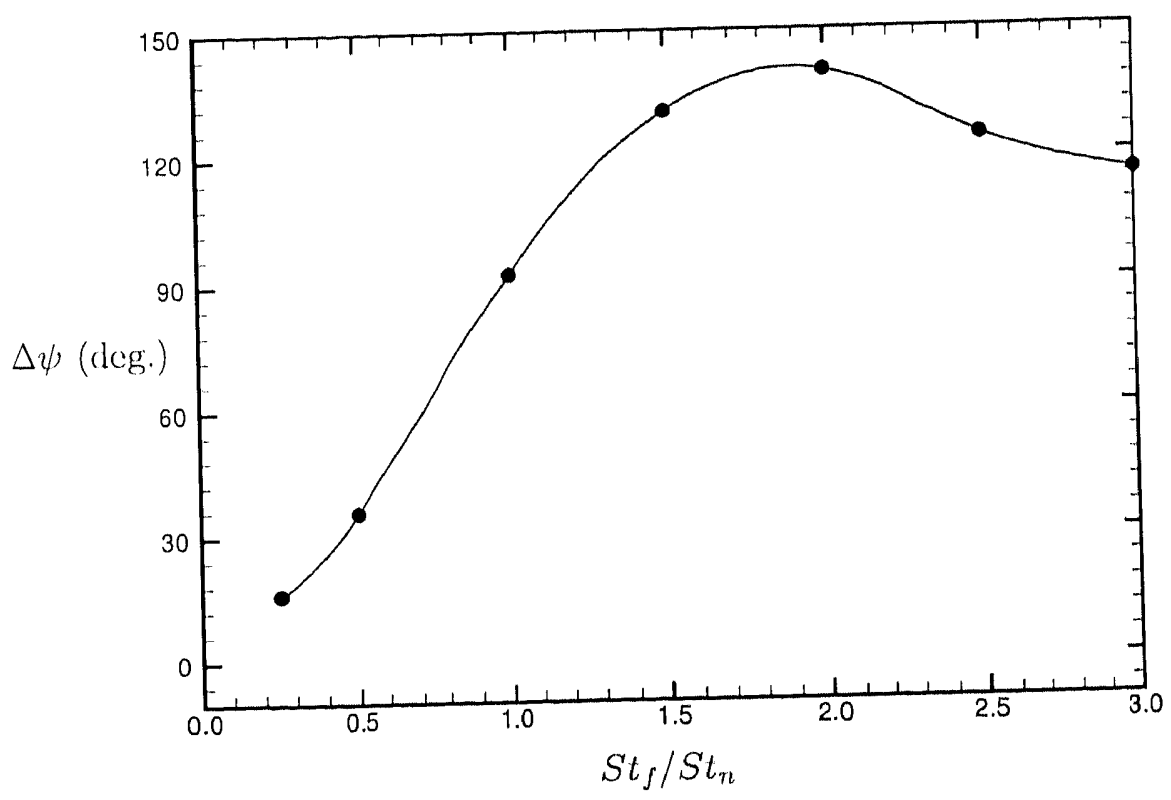

Figure 6.4: Phase angle between the oscillatory forcing and the lift cocflicicnt, $\Delta \psi$, versus the forcing frequency ratio, $S t_{f} / S t_{n}$. 


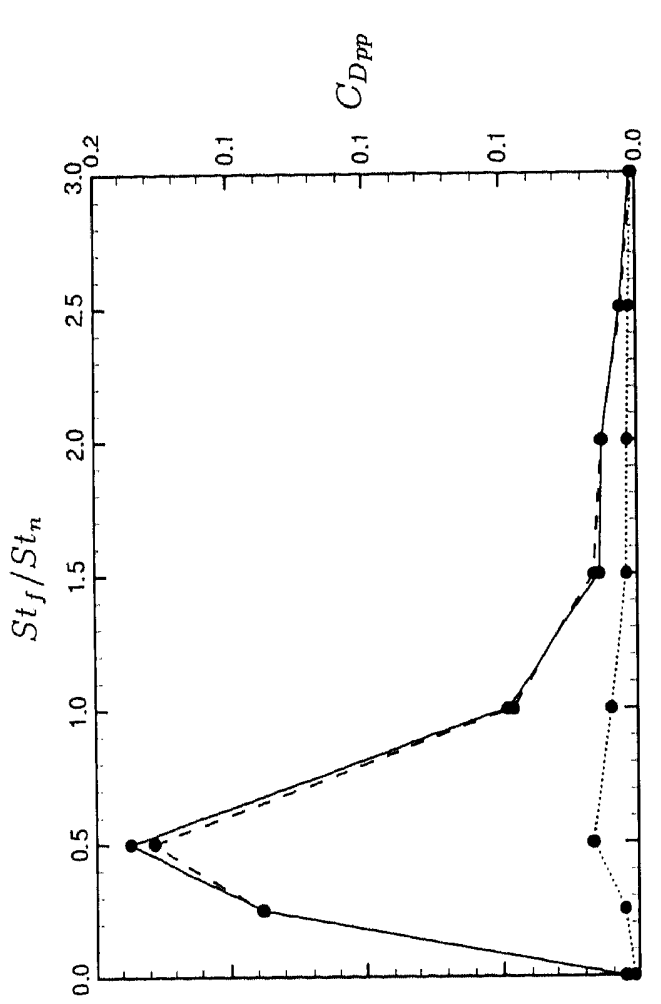

$U^{\frac{+}{2}}$

苞
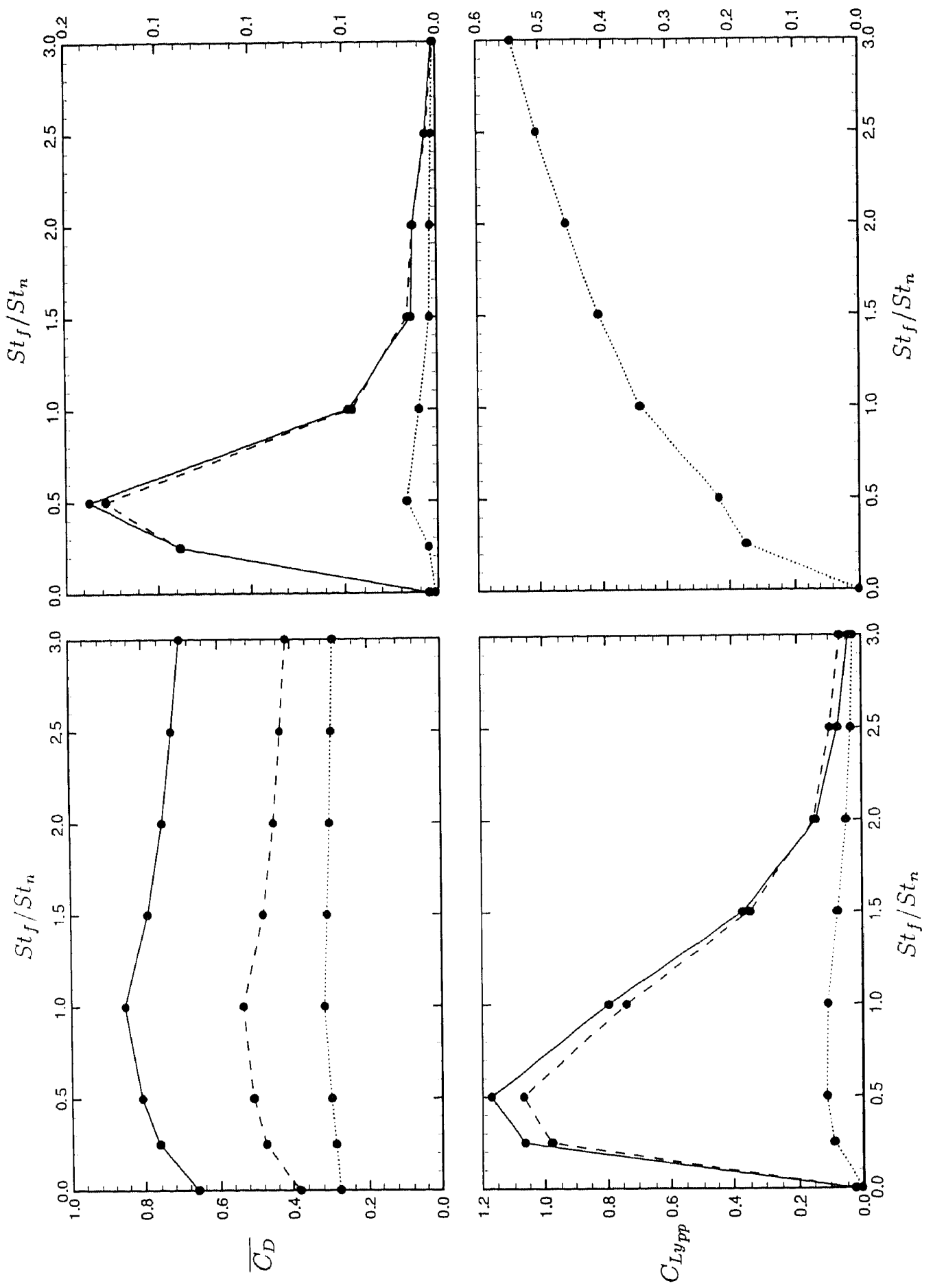

莺

है

$\dot{0}$

运

桴

究

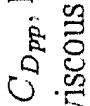

范

过

bo 范

t)

屯ี

के

莎

这

ซ

|

苞

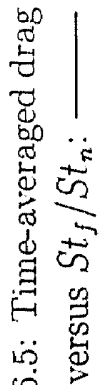

营迢 


\subsection{Vortical wake structure}

In this section the evolution of the vortical wake is described for each of the forcing frequency ratios simulated. In figure 6.6 iso-surfaces of $z$-vorticity are shown at a time instant for which the lateral lift force is at a local maximum. The structures are plotted over a grid to help illustrate scalc. The most obvious effect of an increase in the forcing frequency is the lateral expansion and subsequent contraction of the wake structures. The latcral deflection appcars to be most prominent for $S t_{f} / S t_{n}=0.50$, which coincides with a peak in the fluctuating compononts of the lift and drag forces presented in $\$ 6.1$. For higher $S t_{f} / S t_{n}$, the lateral expansion of the wake is prugressively reduced, such that for $S t_{f} / S t_{n} \geq 2.50$ structures of alternate sign and orientation are convected downstream in an almost parallel strcam.

The expansion and contraction of the wake is further demonstrated in figure 6.7, which presents the timc-averaged $x$-vorticity on the $(y, z)$-plane. The contours range from -20 to 20 in increments of 0.5 , with dashed lines used to indicate negalive values and the zero contour omitted for clarity. For a stationary sphere the wake comprises two threads of oppositely signed vorticity, fed from vorticity generated at the surface. The flow shows an asymmetry with the negative vorticity thread extending further downstream than the positive thread. This flow structure has been described in chapter 5. Oscillatory forcing causes the wake to assume an antisymmetric pattern for all frequencies considered. With increasing forcing frequency, the time-averaged vorticity layers become increasingly deflected away from the streanwise axis. The maximum deflection occurs in the vicinity of $S t_{f} / S t_{n}=1.00$, for which the time-averaged drag coeflicient was found to be at a maximum. The vorticity tails also retract upstream, which is indicative of a greater mixing in the far-wake. For $S t_{s} / S t_{n}>1.00$, the wake progressively narrows and the time-averaged vorticity tails deflect back towards the streamwise axis. The threads also extend further downstream, which is indicative of the more organized antisymmetric array of vortical structures. 

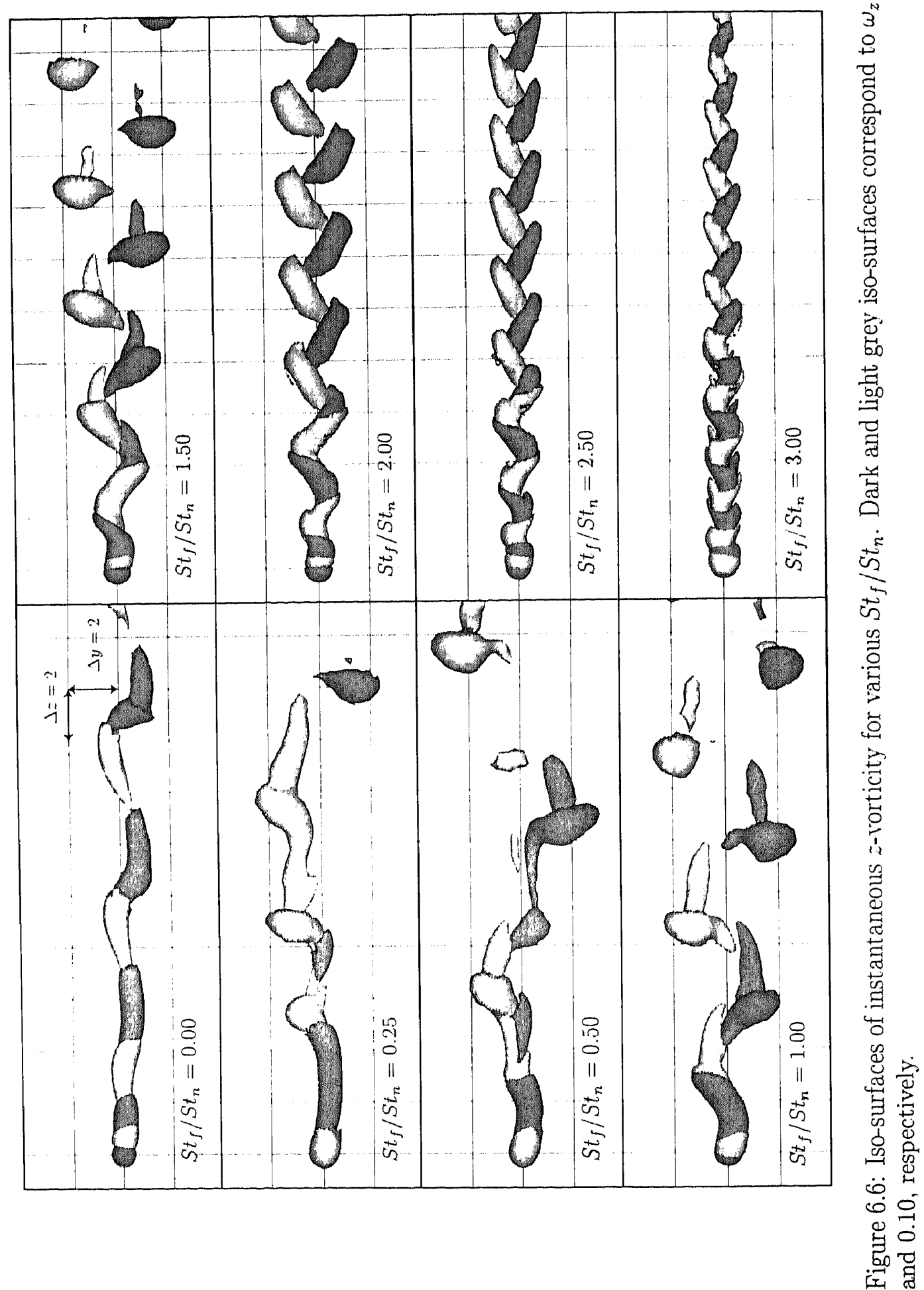


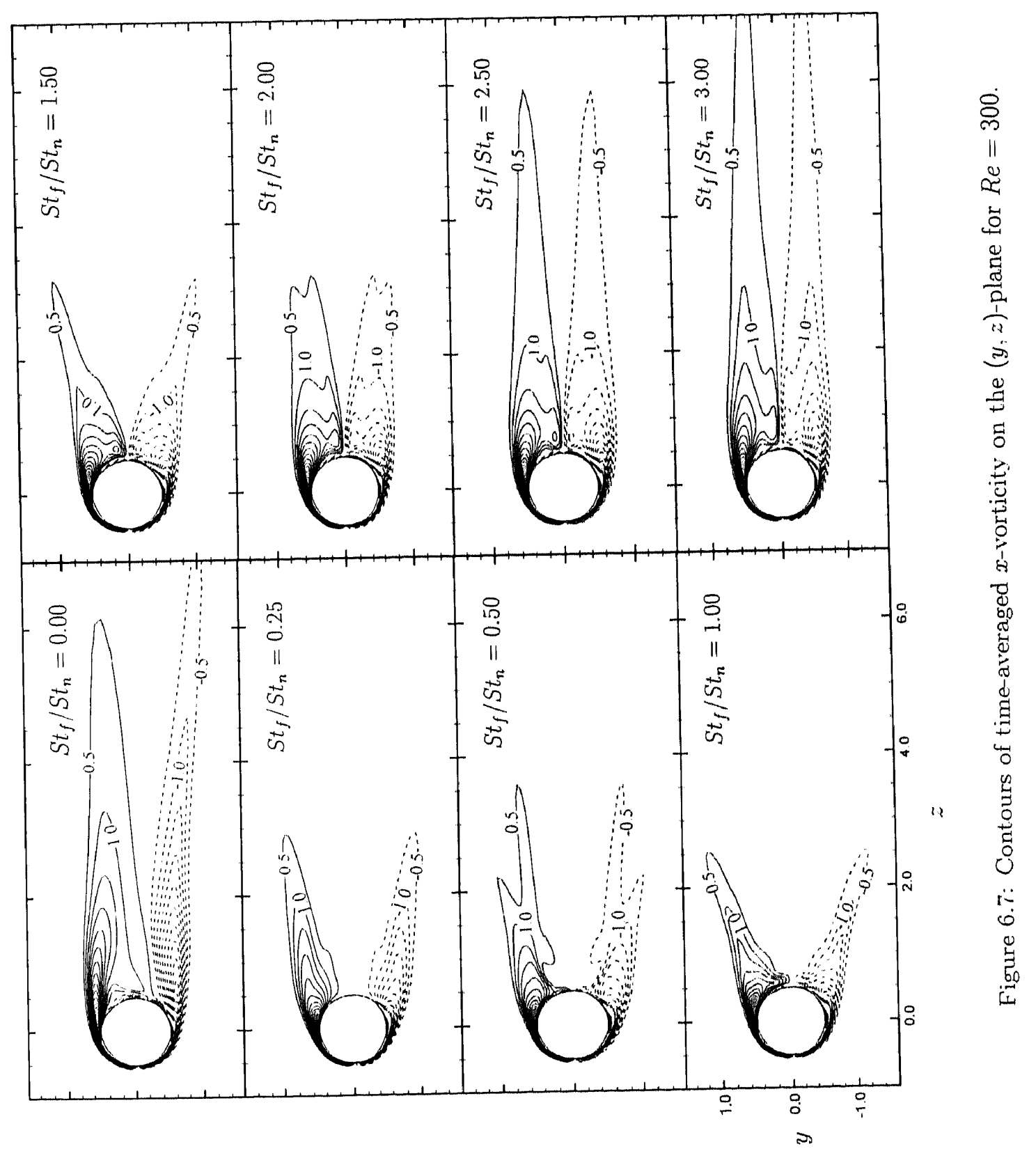


The effect of increasing forcing frequency on the wake structure has been shown to be similar for a two-dimensional cylinder. The numerical simulations of Lu \& Sato (1996) found that, at high forcing frequencics, the wake takes on the appearance of two parallel vortcx arrays. Using flow visualization, the experiments of Tokumaru \& Dimotakis (1991) found that dyc injected upstream of a stationary cylinder clispersed and mixed across the full height of the test section within a fow diamelers downstream of the cylinder. In contrast, under an appropriate forcing, the wake oceupicd approximatcly the same fraction of the test section on exiting the test section as it did in the cylinder near-wake. The size of the discrete structures is also reduced with increasing forcing frequency -... an effect also found in the wake of a two-dimensional cylinder. Cheng et al. (2001b) argued that this must be a result of a decrease in the structure formation time, which becomes synchronized with the forcing frequency.

For $S t_{f} / S t_{n}=0.25$ and $S t_{f} / S t_{n}=0.50$, a number of vortical structures of sume sign and orientation are shed and convect downstream as a 'packet', before a vorticily packet of opposite sign is shed. However, for $S t_{f} / S t_{n} \geq 1.00$ indiviclual structures of altomating sign and orientation are released consecutively. $\Lambda$ higher forcing frequencies the wake structure persists far clownstream. To gain further insight into the mechanisms that shape the wake, the tomporal evolution of the vortical wake is presented in figures 6.8 to 6.14 for each of the forcing frecuency ratios simmlated. For this purpose, the $\lambda_{2}$ structure identification method of Jeong \& I Iussain (1995) is employed by plotting iso-surfaces at a threshold value of $\lambda_{2}=-8 \times 10^{-4}$. To inclicate length scale, the wake cross-sections are marked at streamwise increments of $\Delta z=2$, starting at $z=0$. In figure 6.1, the time instants for which the wake structure is presented are indicaled by a series of dots on the sphere angular velocity profiles. Starting at the time instant where the sphere angular velocity is at a maximum (denoted as $l_{0}^{*}$ ) the wake structure is presented for one full period $(T)$. Here the time variable $t_{c}^{*}=t^{*}-t_{o}^{*}$ is introduced for convenience. For $S t_{f} / S t_{n}=0.25$ the wake structure is presented at time increments of $T / 16$, while at higher forcing frequency ratios a time increment of 
$T / 8$ was decmed sufficient to track the development of the wake.

The temporal evolution of the vortical wake for $S t_{f} / S t_{n}=0.25$ is presented in figure 6.8. At the start of the forcing cycle a hairpin structure $(A)$ has been shed, but remains connected to the near-wake by its legs. In all cases, structures relcased from the underside of the sphere $(y<0)$ comprise predominantly negative $x$-vorticity, while those shed from the upper-side of the sphere comprise predominantly positive $x$-vorticity. To aid description, structures shed from the underside and upper-side will subsequently be referred to as 'negative structures' and 'positive structures', respectively, in reference to the sign of their $x$-vorticity. As this negative structure is convected downstream, it undergoes a reconnection midway along its streamwisc extent to form a sccondary hairpin (B). This secondary structure becomes tilted away from the streamwise direction and assumes a $\Omega$-shape, while the primary hairpin $(A)$ maintains its strenmwise orientation as it travels downstream. In chapter 5, a similar development was found for the hairpin structures shed from a stationary sphere.

A further two like-signed hairpin structures are shed at approximately $t_{c}^{*}=3 T / 16$ and $t_{c}^{*}=5 T / 16$ (structure $C$ and $D$, respectively). At the latter time instant the sphere angular velocily has changed sign and the structure enveloping the sphere begins to reorient itself as $x$-vorticity of positive sign becomes concentrated on the upper-side of the sphere. Despite the sign change in the angular velocity at $l_{c}^{*}=4 T / 16$, the phase lang between the forcing and the response of the wake means that a negative strueture (D) continues to grow and be shed. At $t_{c}^{*}=7 T / 16$, the head of a positive structure (E) has been released into the near-wake and the vortex shedding process described above is repeated, such that a packet of three positive hairpin structures is created and released. Unlike the lirst structure shed in the cycle, which evolves to form a secondary hairpin, the subsequent like-signed structures that are created do not undergo a reconnection. It was noted in chapter 5 that the reconnection requires a structure of sufficient strength and it would appear that these trailing hairpins are of lesser strength than the one that leads the packet. The power density spectrum of the lift coefficient time-history and of 
a velocity trace taken at $(x, y, z)=(-0.170,-0.170,1.492)$ show a peak at the forcing frequency and a second lesser peak at approximately 3 times the forcing frequency. This higher frequency relates to the frequency at which the individual vortical structures are shed.

The temporal cvolution of the vortical wake for $S t_{f} / S t_{n}=0.50$ is presented in figure 6.9. In this case, the peak lift force lags the peak angular velocity by approximately $\Delta \psi=36^{\circ}$. The shedding cycle is most clearly tracked starting at, $l_{c}^{*}=T / 8$, where the lift force is close to a local maximum. At this instant a negative vortical structure $(\Lambda)$ is about to separate from the shroud that envelopes the sphere. This structure shows a pair of downstream protuberanecs which, in the time interval spanning $l_{c}^{*}=T / 8$ to $t_{c}^{*}=2 T / 8$, become elongated and reconnect to form a secondary hairpin (B) downstrcam of the primary structure relcased from the surface. As the primary structure $(\Lambda)$ is convected downstream it becomes tilted away from the streamwise direction, while the secondary structure maintains its streamwise orientation. $\Lambda t t_{c}^{*}=3 T / 8$, a second like-signed structure $(C)$ begins to grow and detaches from the surface by $t_{c}^{*}=4 T / 8$. At this instant the shroud cnveloping the sphere beings to grow over the upper-side of the sphere as positive $x$-vorticity becomes coneentrated there. This marks the start of the shedding of positive structures. Unlike the wake evolution for $S t_{f} / S t_{n}=0.25$, where packets of three like-signed structures are alternately shed from the sphere, at this higher forcing frecuency two like-signed structures are sheed per half-cycke.

The temporal evolution of the vortical wake for $S l_{f} / S t_{n}=1.00$ is presented in figure 6.10. In this case, the peak lift fore lags the peak angular velucity by approximately $\Delta \psi=90^{\circ}$. The lift coefficient is in the vicinity of a local maximum at $l_{c}^{*}=2 T / 8$, at which time the angular volocity is zero. At this time instant, a negative structure (A) has formed on the underside of the sphere and separates from the surface at approximately $t_{c}^{*}=4 T / 8$, but remains attached to the surface through its legs. As this structure grows and begins to convect downstream, it develops protuberances at its downstream end. These serve as the nucleus for the formation of a secondary 
hairpin (B). The process by which this secondary structure is formed is similar to that described for $S t_{f} / S t_{n}=0.50$. Oscillatory forcing at the natural frequency of vortex shedding results in solitary altcrnately signed structures bcing released downstream.

For $S t_{f} / S t_{n}=1.50$ the temporal cvolution of the wake is presented in figure 6.11. The wake rescmbles that at $S t_{f} / S t_{n}=1.00$, but the secondary hairpins (such as structure B) are significantly smaller and appoar to dissipate more rapidly as they travel downstream. On release from the surface, the primary hairpin structures (structure $A$ for example), tilt away from the strcamwise direction. As they travol downstream they quickly assume a $\Omega$-shape and the legs undergo a reconnection to form a scries of vortex loops, such as that labelled C. The evolution of the vortical wake for $S t_{f} / S t_{n}=2.00$ is presented in figure 6.12. The secondary hairpins, which in the provious figures have been shown to diminish with increasing forcing frequency, are now completely absent. The wake comprises an organized array of vortex loops, which maintain their orientation and persist far downstream. Under this forcing condition, the phase difference between the angular velocity and the wake response is in the vicinity of a maximum $\left(\Delta \psi \approx 141^{\circ}\right)$; the lift fore being close to a maximum when the angular velocity is zero. At this instant, the angular acceleration is at a maximum and this is expected to result in a higher production of vorticity at the surface and therefore a higher lift force coeflicient.

The evolution of the vortical structure for $S t_{f} / S t_{n}=2.50 \mathrm{is} \mathrm{presented} \mathrm{in} \mathrm{figure}$ 6.13. An increase in the forcing frequency maintains the basic structure described for $S t_{f} / S t_{n}=2.00$, while the lateral expansion of the wake is reduced further. The higher forcing dictates that the vortex loops are formed and relcased at a faster rate. This results in a closer strcamwise packing of the wake structures and allows for a greater interaction between the consecutive vortex loops. In the near-wake it is evident that a number of consecutively shed structures remain connected to the structure enveloping the sphere to form a 'daisy-chain'. At $t_{c}^{*}=0$ for example, the hoad of structure $A, B$ and $\mathrm{C}$ have detached from the surface, but remain connected to it via their legs. As a result, 
the legs of each hairpin protrude through the oppositely signed $\Omega$-shaped vortex loop formed during the previous half-cycle. As the structures are convected downstream, mutual induction causes their legs to reconnect and form a single appendage, which protrudes through the plane of the oppositely signed vortex loop formed during the subscquent half-cycle. If, for example, the cvolution of structure $\mathrm{A}$ is tracked, it is evident that the legs begin to unite in the vicinity of $t_{c}^{*}=4 T / 8$ and take the form of a single appendage (D) for $t_{c}^{*} \geq 7 T / 8$.

The temporal evolution of the vortical wake for $S t_{f} / S t_{n}=3.00$ is presented in figure 6.14. Once a hairpin structure becomes detached from the surface, it interacts with the oppositely signed structures shed at the previous and subsequent half-cycles. If we consider the instant $t_{c}^{*}=0$, for example, the head of the positive structure (B) forms a bridge over the legs of the negative structure $(A)$, shed at, the previous half-cycle. In time, their mutual induction draws the legs of the negative structure towards the hearl of the positive structure until they intersect. This is followed by two interesting elfects. An cnlarged view of the near-wake system of vortical threads for $l_{c}^{*}=0$ is shown in figure 6.15. The legs of the negative hairpins, for example the structure denoted C, are expected to induce a negative $y$-volocity along the flow symmetry plane. This appears lo be what causes the positive structures to dip on the symmetry plane, such that further downstream they lake a cardioid-shapo (as illustrated by structure E). The legs of the positive and the negative structures, D and C for example, lie approximalely parallel, cansing the weaker negative legs to diffuse rapidly, such that they are absent further downstream. Returning to figure 6.14 , it is evident that the far-wake does not comprise a series of discrete vortex loops as is found at lower Stg/St $t_{n}$, instead the structures coalesce and diffuse more rapidly. Despite the greater mixing, the far-wake does not show a noticcable increase in lateral cxpansion.

The downstream expansion of the wake becomes more apparent by plotting the time-averaged velocity profiles on the symmetry plane at scveral stations downstream of the sphere. Figure 6.16 presents the time-averaged strcamwise velocity profile at 


\subsection{VORTICAL WAKE STRUCTURE}

stations $z=2.50$ and 5.00. The wake profile for a stationary sphere $\left(S t_{f} / S t_{n}=0.00\right)$ shows the greatest velocity deficit. Rotary oscillation increases the entrainment of frecstream fluid into the ncar-wake, such that the peak velocity deficit is reduced and the width of the wake shows a more pronounced expansion with downstream development. The wake width is maximum for $S t_{f} / S t_{n}=1.00$, reiterating the obscrvations made from figure 6.7, where the time-averaged shear layers show the greatest lateral deflection. At this forcing condition the velocity profile at $z=5.00$ shows two local velocity minima, due to the wake structures convecting downstream in two streams staggered away from the streamwise axis. With increasing forcing frequency, these move towards the wake centreline to form a single minimum, and the peak velocity deficit increases. For $S t_{f} / S t_{n}=2.50$ and 3.00 , it is evident that the wake does not undergo a noticcable expansion with downstream development.

Finally, figure 6.17 presents the time-averaged streamwise velocity along a radial cut cmanating from the downstream pole of the sphere $(\theta=0)$. The absence of a reverse flow region for $S t_{f} / S t_{n}=0.25$ and 0.50 is particularly striking. An examination of the instanlaneous streamwise velocity shows that a recirculation region exists only while the first structure in a packet of like-signed structures is developing in the near-wake. This is illustrated in figure 6.18, which prosents the streamwise velocity time-history at two points along the clownstream pole for $S t_{f} / S t_{n}=0.25$. The dots indicale the lime instants for which the instantancous wake structure is presented in figure 6.8 . The peak reverse flow velocitics occur near $t_{c}^{*}=7 T / 16$ and $t_{c}^{*}=15 T / 16$, which mark the start of the shedeling of negative and positive structures, respectively. Once the first structure in a like-signed packet is formed and begins to convect downstream, the streamwise velocily quickly rises to a positive value such that a reverse flow exists for a lesser fraction of the overall shedding cycle and therefore a recirculation region is absent from the time-averaged velocity. Returning to figure 6.17, as the forcing frequency is increased further, the recirculating region grows progressively but, over the range of $S t_{f} / S t_{n}$ considered, remains smaller than that for a stationary spherc. In contrast, 
the expcriments of Taneda (1978a), which applied a constant amplitude forcing to a two-dimensional cylinder, found that as the forcing frequency is increased the wake narrows and, above a critical value, the recirculation region is completely absent. 


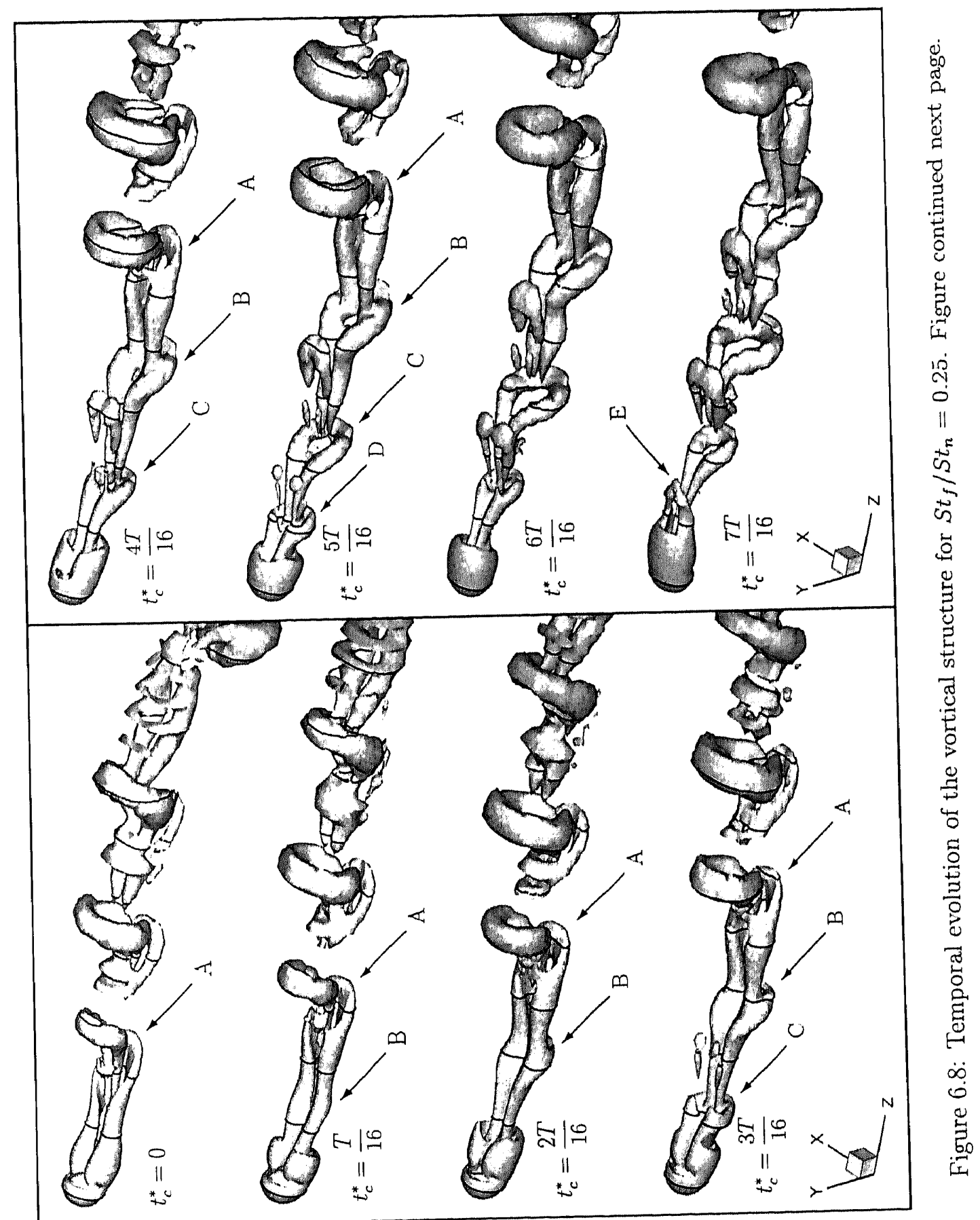




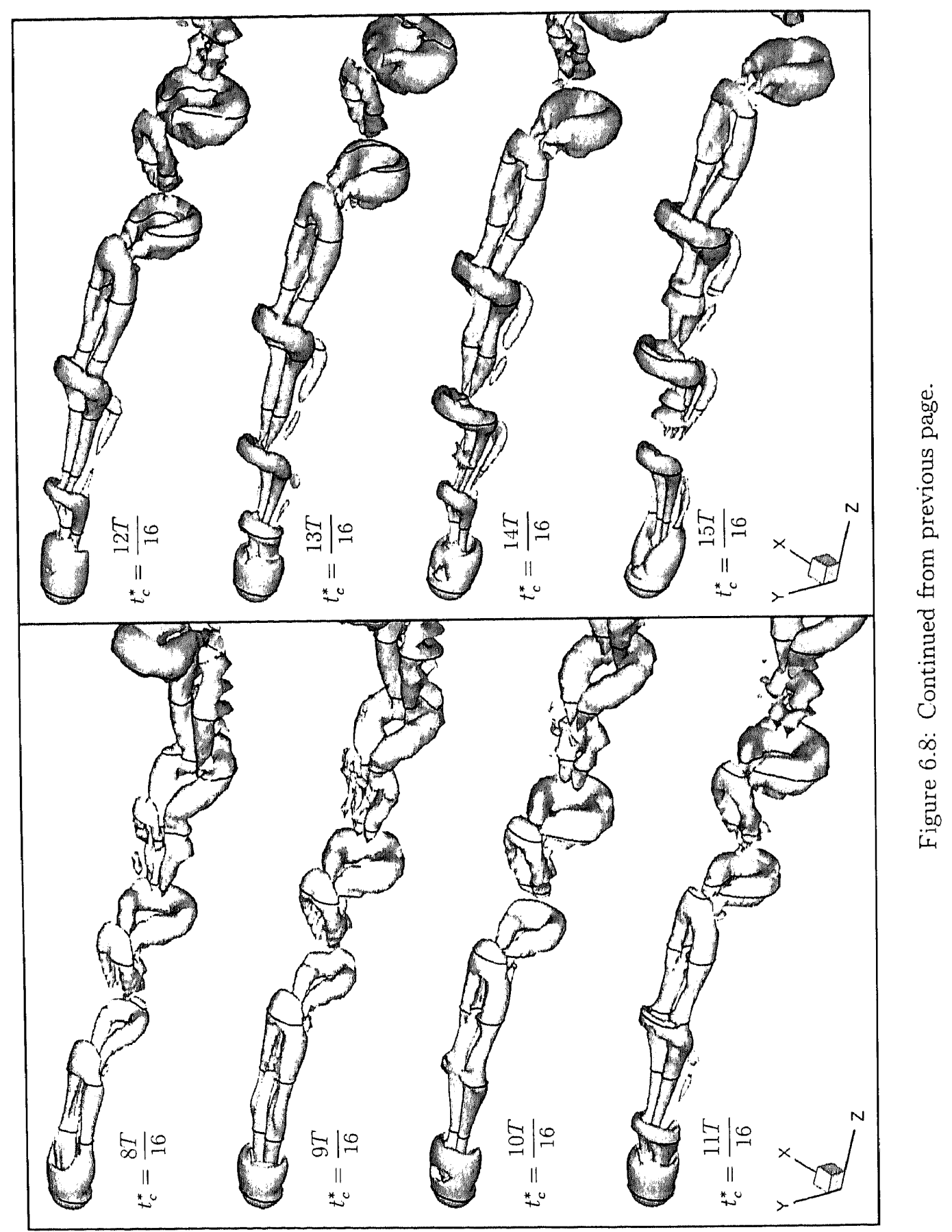




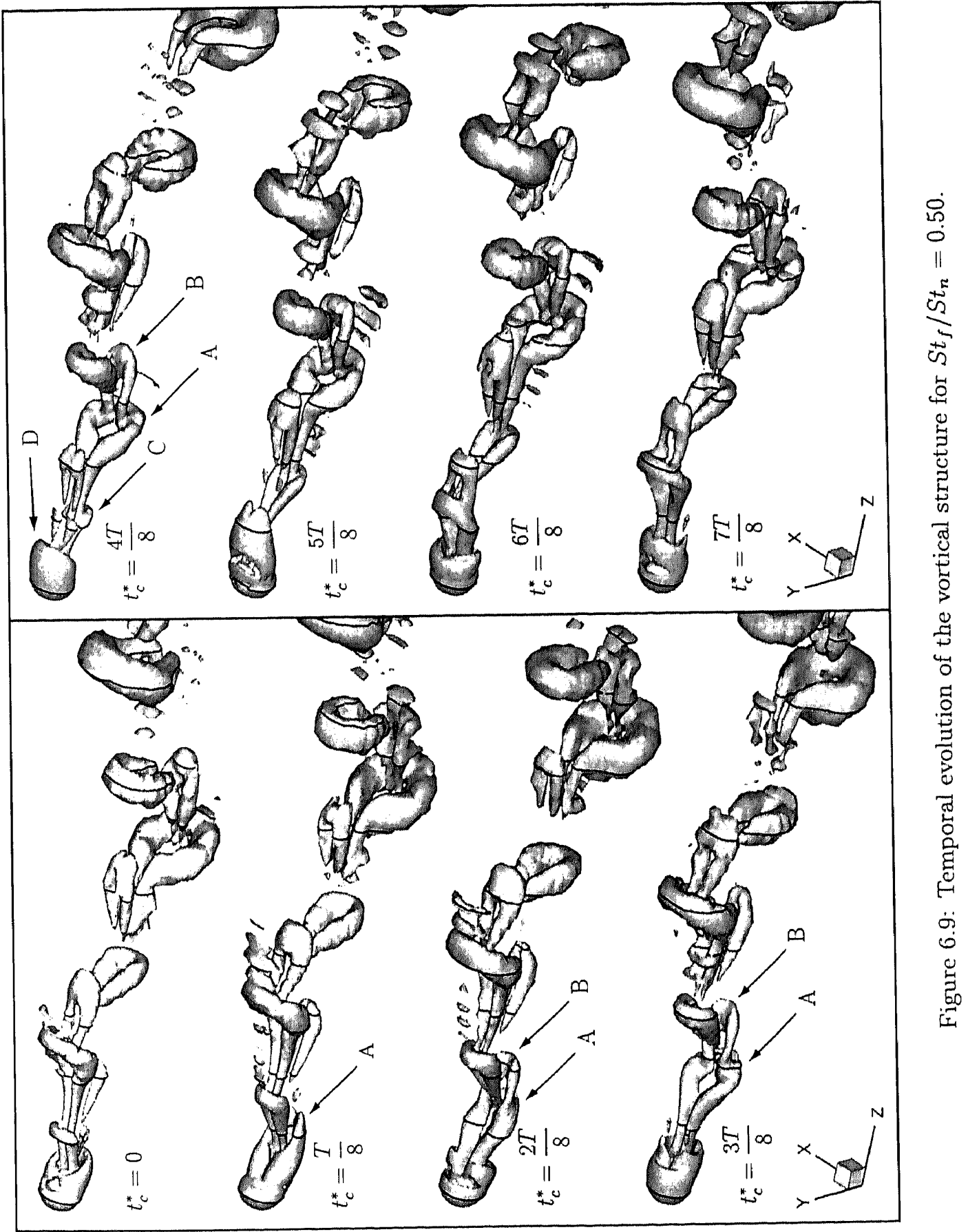




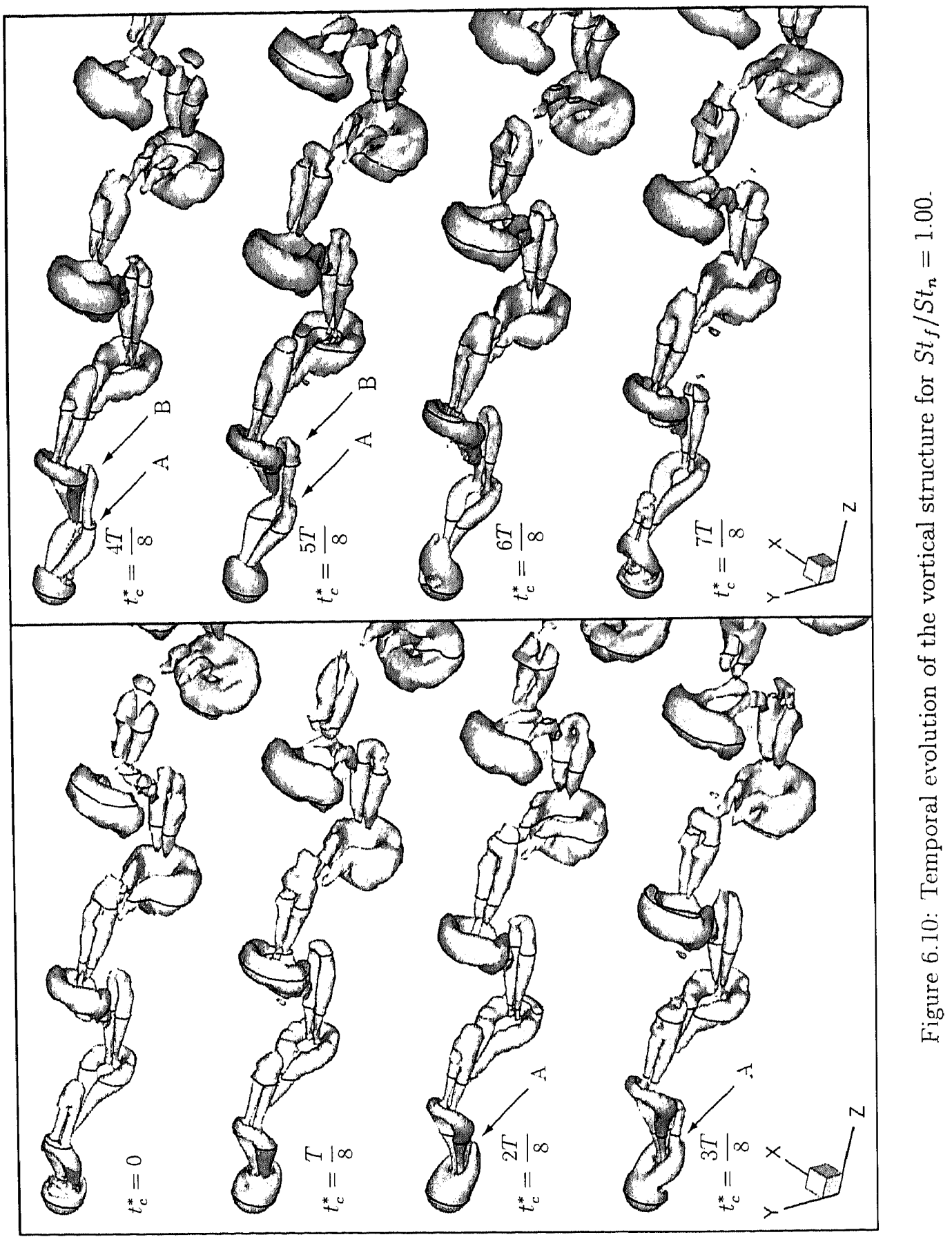




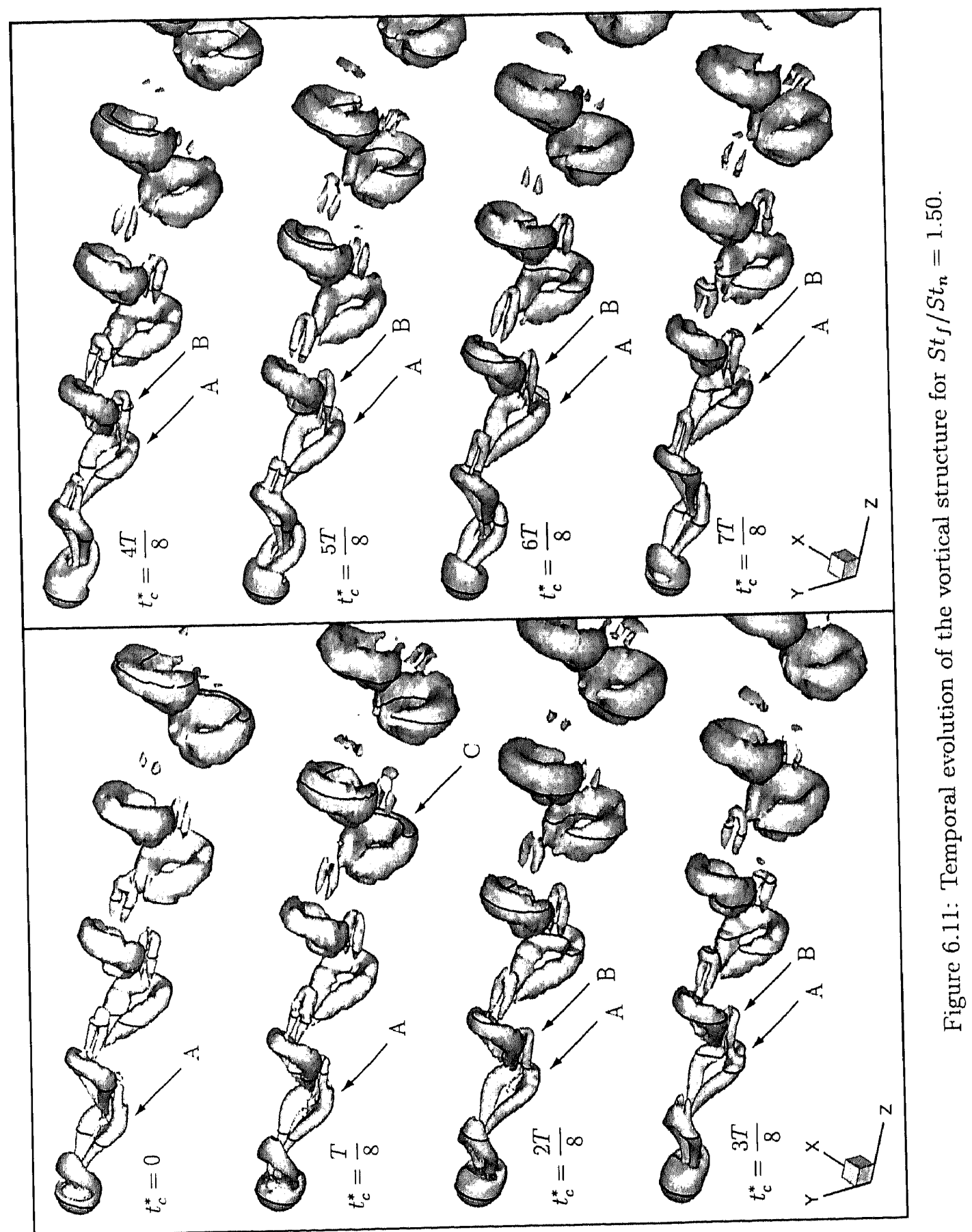




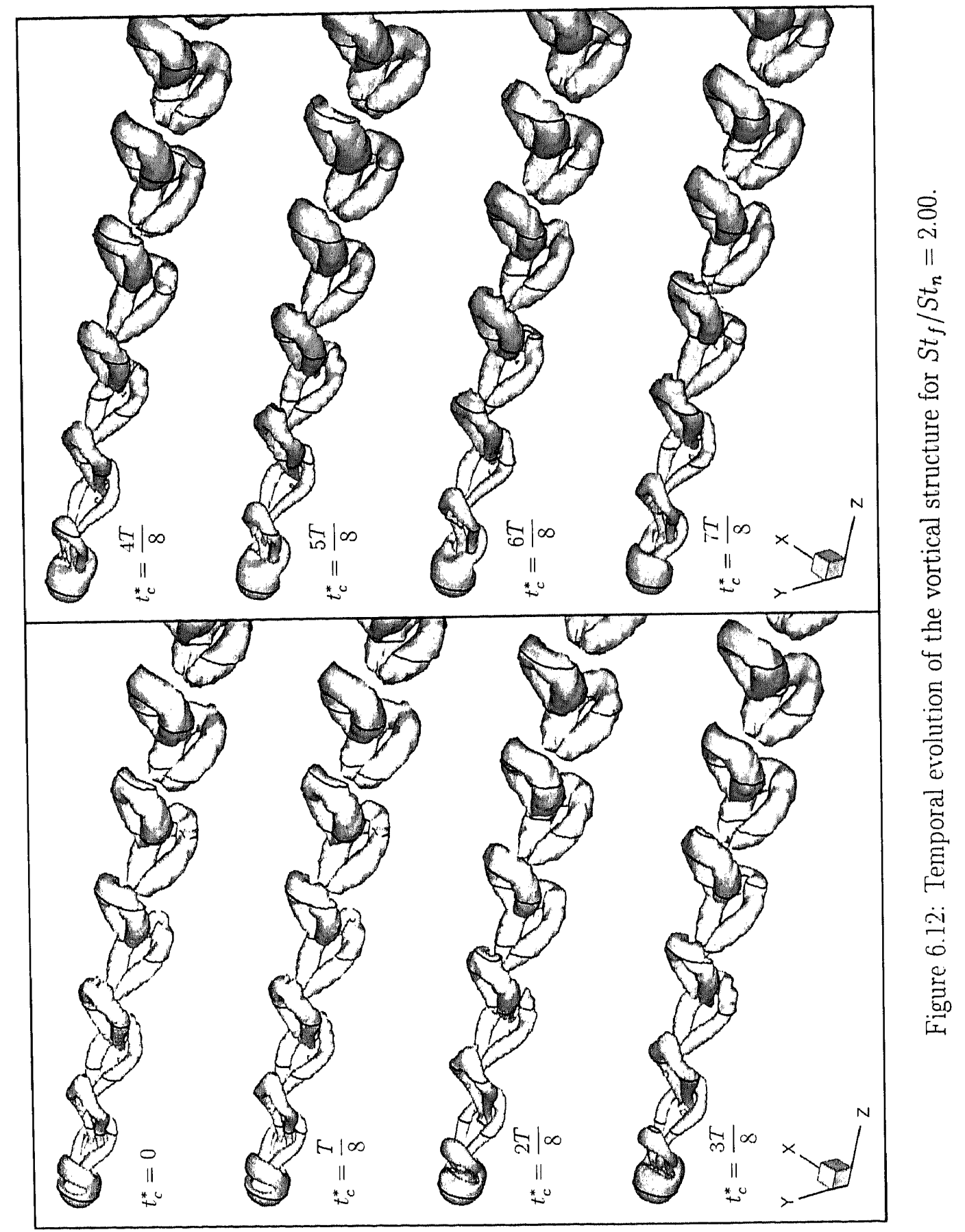




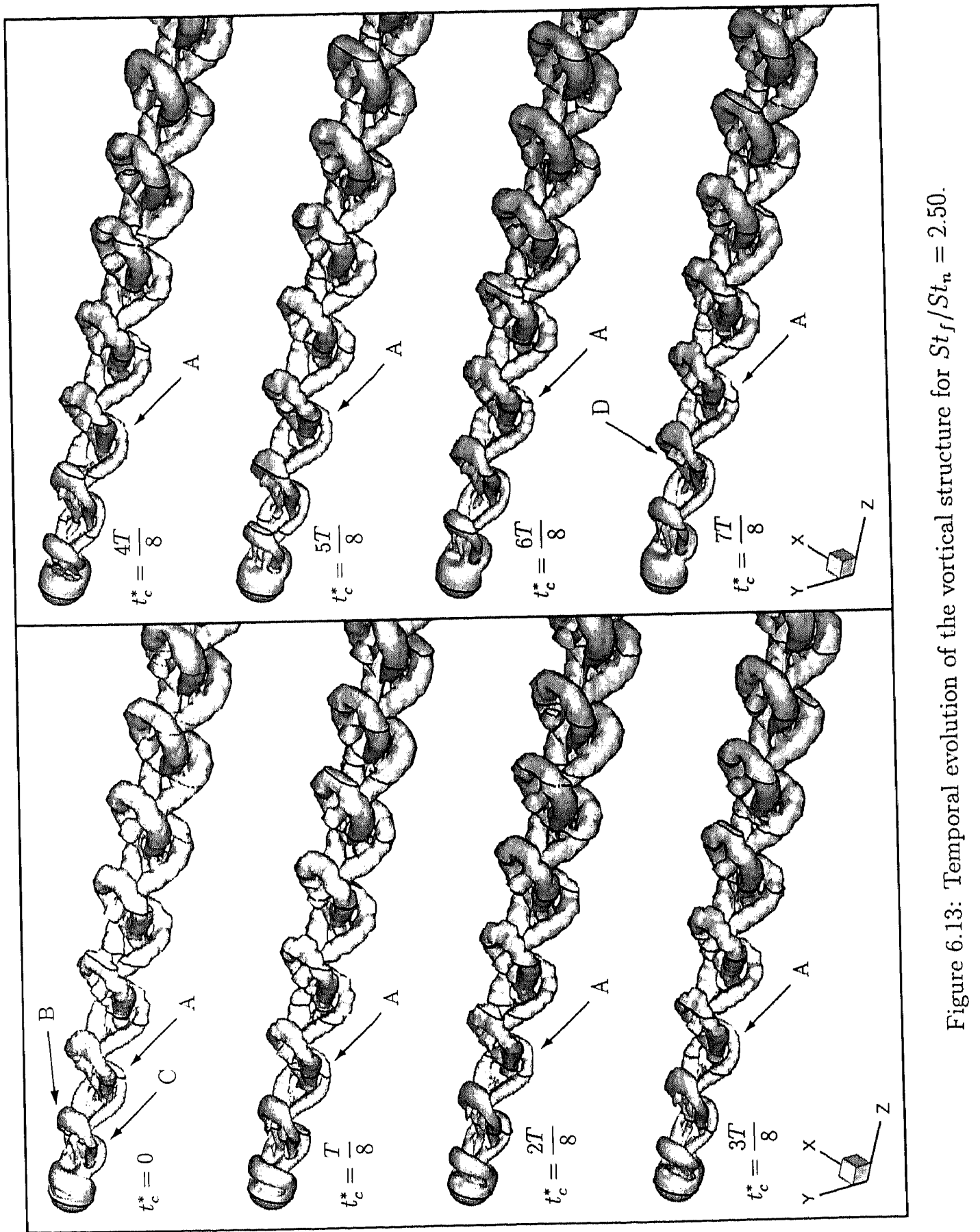




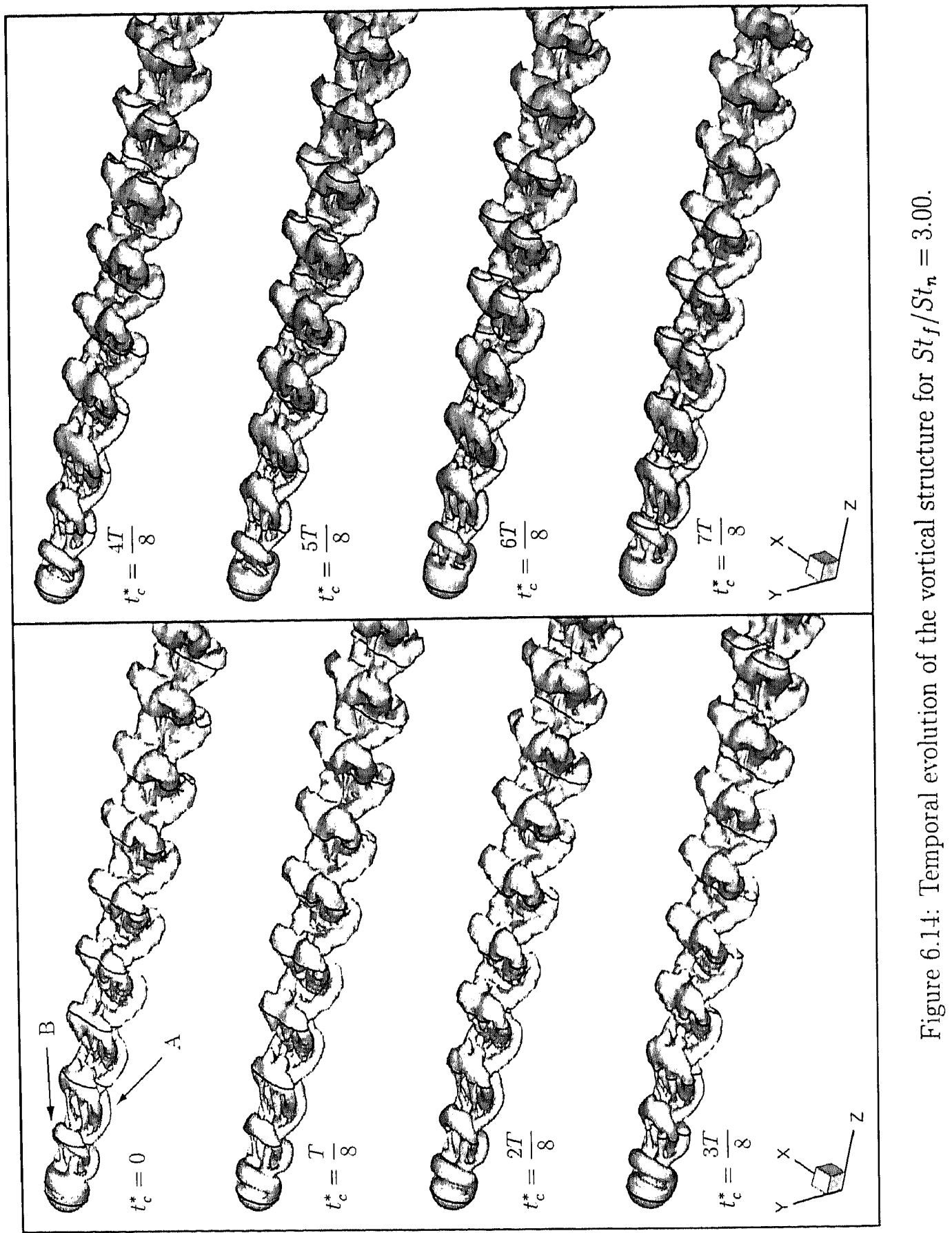



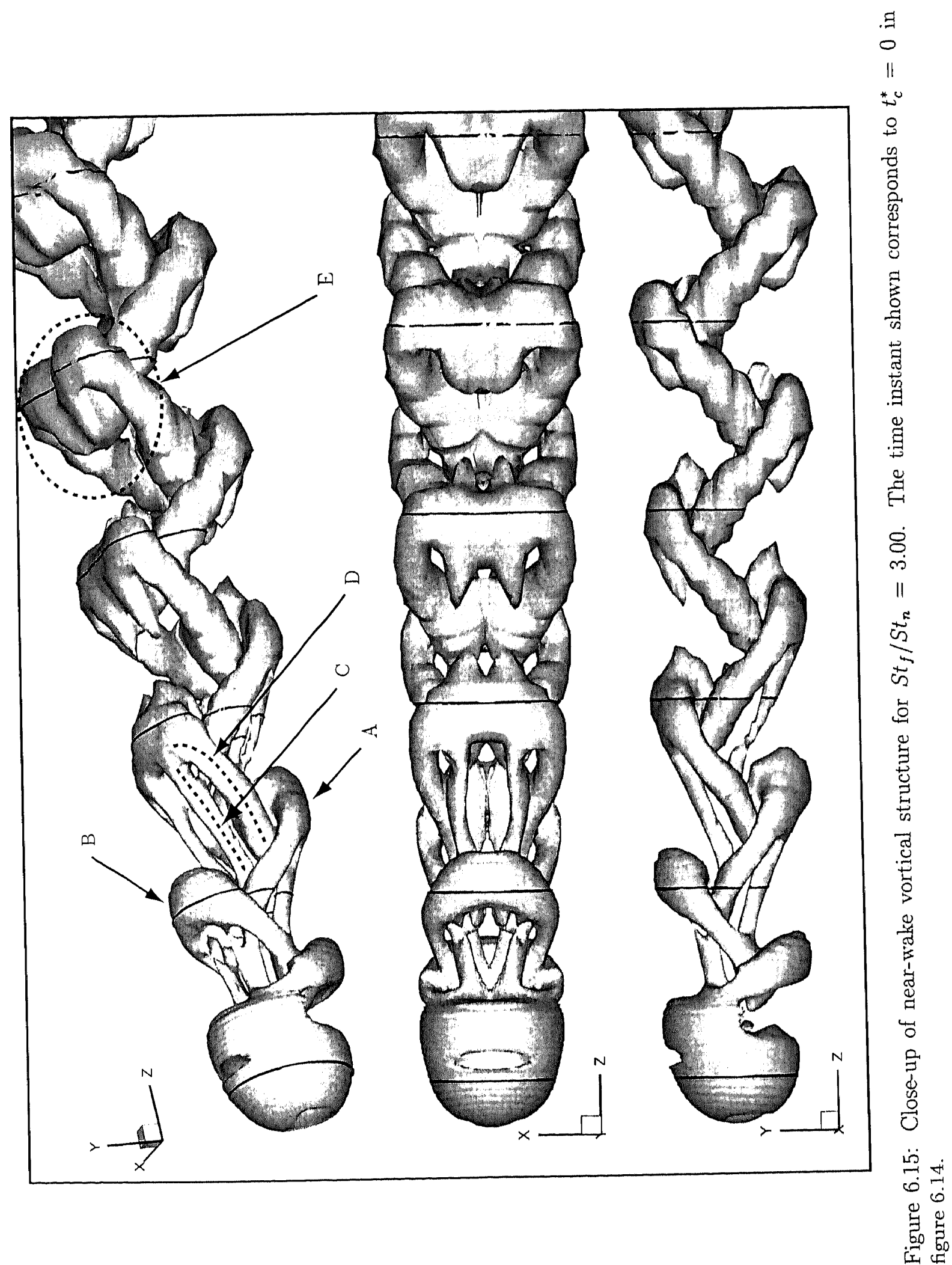

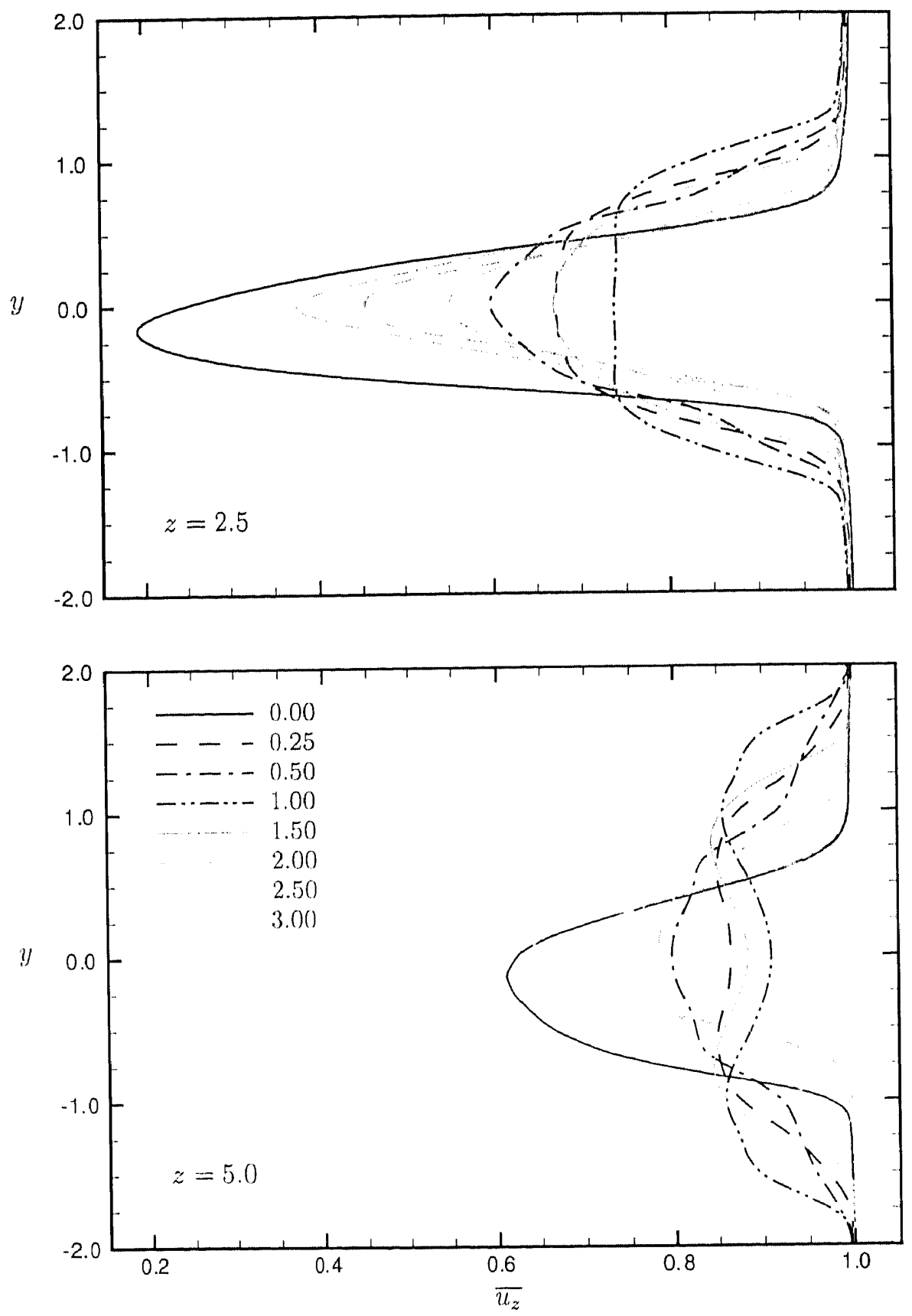

Figure 6.16: Profiles of $\overline{u_{z}}$ at $z=2.5$ and $z=5.0$ for various $S t_{f} / S t_{n}$. Legend refors to $S t_{f} / S t_{n}$ 


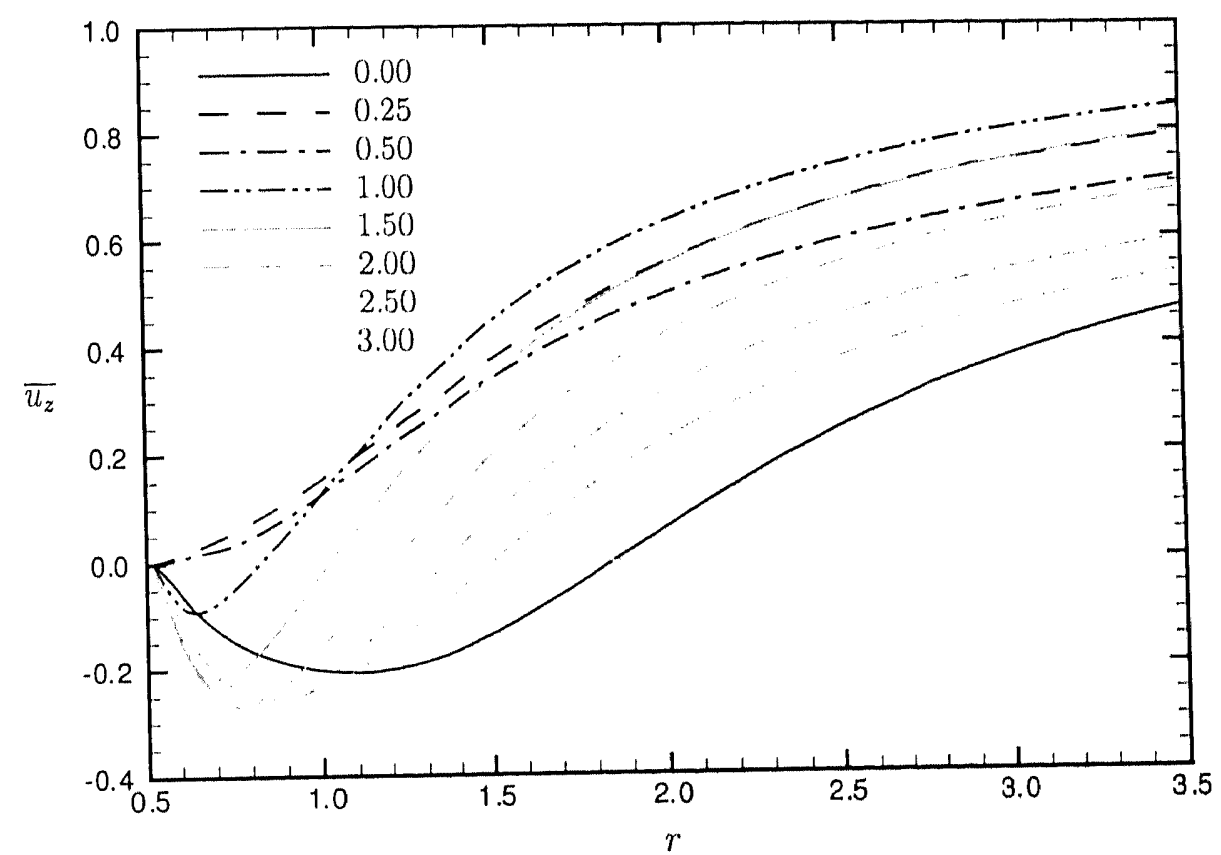

Figure 6.17: Profiles of $\overline{u_{z}}$ along the downstream pole $(\theta=0)$ for various $S t_{f} / S t_{n}$. Legend refers to $S t_{f} / S t_{n}$.

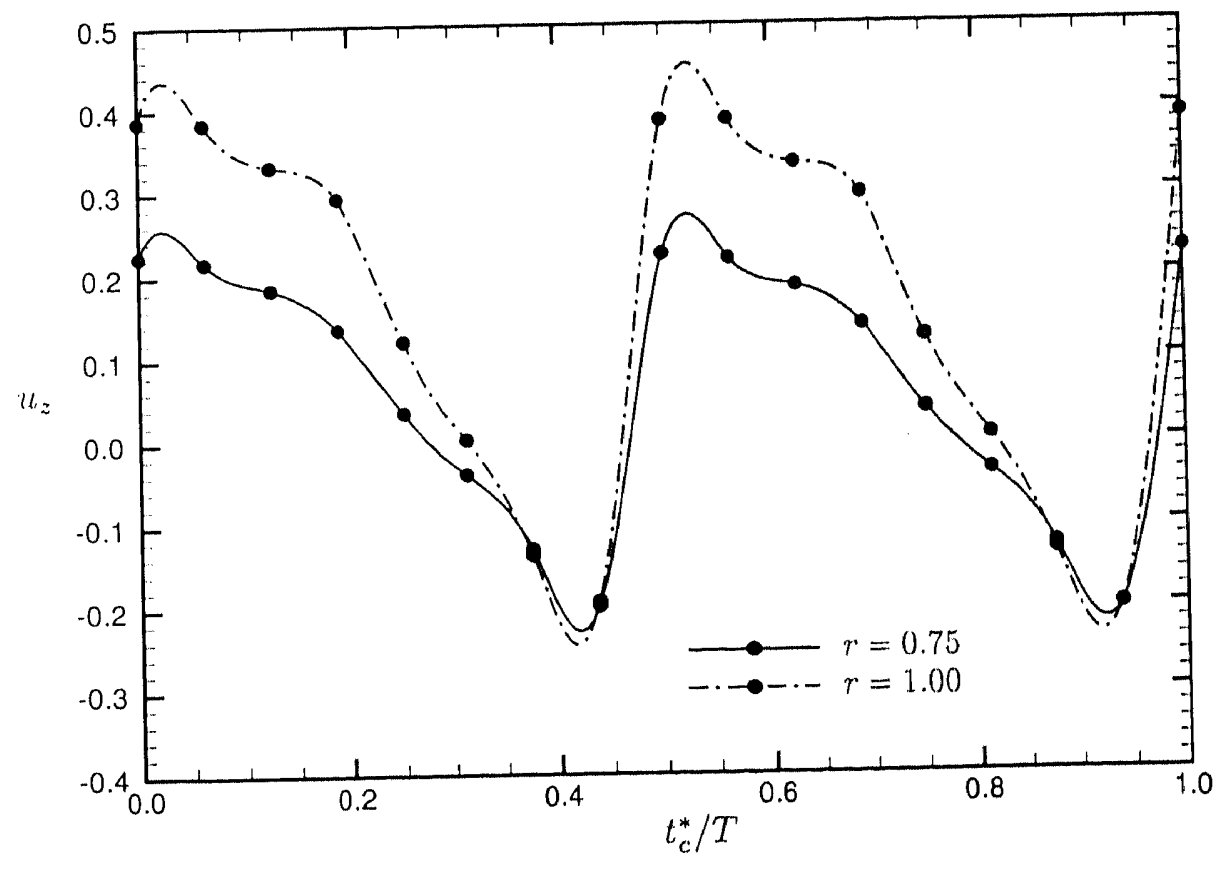

Figure 6.18: Streamwise velocity time-history at the points $(r, \theta, \phi)=(0.75,0.00,0.00)$ and $(1.00,0.00,0.00)$ for $S t_{f} / S t_{n}=0.25$. 


\subsection{Summary}

The flow past a sphere undergoing oscillatory transverse rotation was numerically simulated for a frecstream Reynolds number of $R e=300$. A sinusoidal forcing with an angular velocity amplitude of $\Omega_{\circ}^{*}=0.50$ was prescribed, and forcing frecluencies in the range of 0.25 to 3.00 times the natural frequency of vortex shedding were considered. Particular emphasis was placed on describing the cvolution of the vortical wake structure, as identified using the method of Jeong \& Hussain (1995). Despite the limited scope of this study, a number of interesting findings have cmerged.

Oscillatory forcing leads to a two-sided shedding of alternately signed hairpin structures, such that the drag oscillates at twice the frequency of the lift. Over the forcing frequency range considered, the dominant frecuency in the lift history becomes synchronized with the forcing frequency. Their phase relationship varies significantly and is indicative of the changing vortex formation phase with respect to the borly motion.

For $S t_{f} / S t_{n}=0.25$ and 0.50 , the evolution of the vortical wake shows that three and two like-signed hairpin structures are shed per half-cycle, respectively. The lateral deflection of the noar-wake structures is maximum for $S t_{\mathrm{g}} / S t_{n}=0.50$ and correspondingly leads to maximums in the fluctuating amplitudes of the forces. A maximum in the mean drag occurs in the vicinity of $S l_{f} / S t_{n}=1.00$, for which lateral expansion of the time-averaged wake is greated. As the forcing frefunency is incroased further, the moan drang decreases and the fluctualing forces tend towards zero monotonically.

For $S t_{f} / S t_{n} \geq 1.00$, individual struetures of alternate sign and orientation are shed consecutively. As the forcing frecuency is increased, wake synchronization dictates that vortex loops are formed and released at a faster rate. The result is a closer streamwise and latcral packing of the wake structures, which allows for a greater interaction between consecutive structures. The lateral expansion of the wake is progressively reduced, such that for $S t_{f} / S t_{n}=2.50$ and 3.00 , the structures convect downstrean in an almost parallcl array. 


\section{Chapter 7}

\section{Conclusions}

This thesis documents two related numerical studies of the uniform flow past a rotating sphere at moderate Reynolds numbers. The first considers the case of steady rotation about an axis transverse to the freestream flow. This problem has been the subject of a number of carlior numerical and experimental studies. Despite these efforts, little was known about the threc-elimensional nature of the flowficld and the mechanisms that elrive the various wake transitions that are observed as the sphere rotation rate is increased. The moderate Reynolds numbers of 100, 250 and 300 were considered, which for a stationary sphere cover the axisymmetric stcady, asymmetric steady and vortex shedeling regines. The primary objective was to identify wake transitions that occur over the range of rotational speeds $\Omega^{*}=0.00$ to 1.00 , where $\Omega^{*}$ is the maximum velocity on the sphere surface nomalized by the fresstream velocity.

The second study considered the case of sinusoidal rotation about an axis transverse to the freestrean flow. To the anthor's knowledge this problem has never been investigated. For the single Reynolds number of 300 and a forcing amplitude of $\Omega 2_{0}^{*}=0.50$, the exploratory study examined the nature of the wake as the forcing frequency was increased from 0.00 to 3.00 times the natural vortex shedding frequency.

A highly accurate Fourier Chebyshev spectral collocation method was used to solve the time-dependent incompressible Navier Stokes equations directly. The solver was cxtensively validated and shown to accurately capture the flow physics. To facilitate the identification and tracking of vortical structures in the flow, a number of vortex 
extraction methods - which classify the flow locally -... were critically cvaluated. The method judged most suitable for the problem of interest was used exclusively in the subsequent inquiry. The details of this supporting work were provided in chapters 2 , 3 and 4 . The main results for the problem of stcady rotation and oscillatory rotation were summarized at the end of chapter 5 and 6 , while the overall conclusions are as follows.

\section{Steady rotation}

As the rotation rate is increased, the relative frecstream-to-surface velocity is increased on the advancing side of the sphere and decreased on the retreating side. As a result, frecstream fluid becomes increasingly vectored over the lee side of the sphere, and the volume of the recirculating region is progressively reduced until it is completely destroyed for rotation rates higher than $\Omega^{*}=0.30$ to 0.50 . On the advancing side, the higher relative velocity leads to a progressive intensification of the shear layer separating from this side of the sphere. These processes have different effects on the global wake structure depending on the Reynolds number.

For $R e=100$, the initially axisymmetric wake modergecs a transition to a double threaled structure. As the rolation rate is increased a greater circulation is generated, which leads to the intensification of the threads. The flow, however, remains steady. For $R e=250$, sphere rotation triggers a transition to a vortex shechling regime: characterized by the shedding of vortex loops at a single shededing freculuency. Vortex shedding is often described as a process of 'build-up and release' of the fluid within the recirculating wake. As the wake is reduced in volume, this process must oceur on a faster time-scale such that the vortex shedding Strouhal number increases. At higher rotation rates, the recirculating wake is completely absent and the conditions necessary for vortex shedding are climinated. The wake then rescmbles the double-thread structure found at $R e=100$. 
For $R e=300$, the wake of a stationary sphere is unsteady. As the rotation rate is increased beyond a critical valuc, vortex shedding is suppressed via the same mechanism found for $R e=250$. In this case, the shedding of vortical structures returns for $\Omega^{*} \geq 0.80$, albeit in a different form. Unlike the shedding process at lower rotation rates, the discrete vortical structures originate from the roll-up of vorticity along the intense shear layer separating from the advancing side of the sphere. The absence of such an instability at lower Reynolds numbers further supports the instability mechanism being of the Kelvin-Helmholtz type. To the authors knowledge, this second transition has never been observed before. These findings provide important information lowards understanding the process of turbulence enhancement resulting from the presenee of solid particles in a fluid.

The simulation of particulate-laden flows requires an accurate description of the forces and moments experienced by the solid particles. At moderate Reynolds numbers, accurate experimental measurements are difficult due to the small loads experienced by the sphere and the presence of supporting devices. These issues are not present in numerical simulation; however, it was discovered that, previous mumerical studies in the range $R e=100$ to $R e=300$ had incorrectly specified the bounclary conditions on the sphere surface. In light of this, it is the author's understanding that the present study constitutes the first highly accurate numerical investigation of the forces and moments experienced by a transversely rotating sphere in the Reynolds mumber range 100 to 30() .

\section{Oscillatory rotation}

The scope of this exploratory inquiry was limited and served to give some insight into the flow physics and raise further questions to help focus future investigations into this problem. The effect of a sinusoidally varying rotation rate on the evolution of the vortical wake was considered for $R e=300$ and a range of forcing frequencics that 
cncompassed the natural frequency of vortex shedding. Although oscillatory forcing of a cylindrical body has received considerable attention, this investigation is believed to be the first of its kind for a spherical particle.

For the forcing amplitude of $\Omega_{\mathrm{o}}^{*}=0.50$, wake shedding becomes synchronized with the forcing frequency, while the phase relationship between forcing and wake shedeling varies. Oscillatory forcing causes the wake to undergo a two-sided wake shedding, much like the Kármán vortex strect generated downstream of a two-dimensional cylinder at moderate Reynolds numbers. At forcing frequencics less than the natural shedeling frequency, the wake expands laterally with increasing forcing frequency and several likc-signed structures are shed per half cycle. Large lateral deflections of the near-wake are accompanied by fluctuation forees significantly larger than those experienced by a stationary sphere or a sphere in stcady rotation.

At higher forcing frequencies, individual structures of alternate sign and orientation are shed consecutively. As the forcing frequency is increased, wake synchronization dictates that vortex loops are formed and released at a faster rate. This results in a closer streamwise and lateral packing and allows for a greater interaction between consecutive structures. The lateral expansion of the wake is progressively reduced, such that for the highest forcing frequencies tested, structures are convected downstream in an almost a parallel stream. The wake of a circular cylinder undergoing robary oscillation has previously been shown to mudergo analogons changes with increasing forcing freculency.

\subsection{Suggestions for future work}

There are a number of aspects which recuire further investigation to improve our understanding of this complex problem. A series of suggestions are listed below:

- For the case of stcady rotation, a number of topological transitions of the wake were observed. An analysis of the stability of these transitions, using Landau models for example, would serve to gain further insight into the nature of the 
wake. Such a study should also yicld accurate predictions of the transitional values of the rotation rate.

- A natural extension to the study of steady rotation is to consider higher Reynolds numbers. This will necessitate simulations using higher temporal and spatial resolutions. To make such computations feasible, a solver should be developed to run on parallel machines with distributed momory. Also, when the number of collocation points is very large, the Chebyshev collocation method used to calculate radial derivatives is known to degrado in accuracy due to round-off errors. It is advised that this scheme be replaced by an altemative discretization, such as a high-order compact differencing.

- An investigation of the effect of higher rotation rates would also be of interest. For $R e=300$, the second transition to vortex shedeling is consistent with a KolvinHelmholtz instability of the intense shear layer separation from the advancing side of the sphere. It is postulated that when the rotetion rate is sufficiently large, a similar transition should also ocenr for $R e=250$.

- The flow past a sphere in stcady rotation has been studied for two principle directions of rotation: about axes transverse and parallel to the freestrom flow. In working towards muclerstanding the flow aromed particles undergoing more general motion, it would be of value to examine the flowfield for a range of rotation axis oricntations.

- Finally, the study of rotary oscillation was very linited in scope. The Reynolds number, rotation rate amplitude and forcing frecuency parameter space needs to be explored in greater detail. 


\section{References}

AChenBaci, E. (1972a). The effects of surface roughness and tumnel blocknge on the flow past spheres. J. Fluid Mech. 65, 113125.

ACHEnBACI, E. (1972b). Experiments on the flow past, spheres at very high Roynolds numbers. J. Fliluid Mech. 54, 565575.

Achenbacin, E. (1974). Vortex shedding from spheres. J. Fluid Mech. 62, 209221.

Aoki, K., Hiro'ta, E., Nagisse, J., \& Naknyama, Y. (2003). Aorodynamics characteristics and flow pattorn to surface structure of a bascball ball. 7 th Asian Symposium on Visualization.

BAEK, S. \& SUNG, H. J. (1998). Numerical simulation of the flow behind a rotary uscillating circular cylinder. Phys. Fluids 10 (1), 869876 .

BAGCHI, P. \& BALACIIANI)AR, S. (2002a). Elfect of frec rotation on the motion of a solid sphere in lincar shear flow an moderate Re. Phys. Fluids 14 (8), 27192737.

BAGCHII, P. \& B BLACHANDAR, S. (2002b). Stcady planar straining flow past a rigicl sphere at moderate Reynolds number. J. Fluid Mech. 466, 365 407.

Barkia, H. M. \& Auchterlonie, L. J. (1971). The Magmus or Robins effect on rotating spheres. J. Fluid Mech. 47, 427447.

Batchelor, G. K. (2000). An introduction to fluid dynamics. Cambridgo University Press. 
Bell, J. B., Colella, P., \& Glaz, H. M. (1989). A second-order projection method for the incompressible Navicr Stokes cquations. J. Comput. Phys. 85, 257. 283.

BEST, J. L. (1998). The influence of particle rotation on wake stability at particle Reynolds numbers, $R e_{p}<300 \cdots$ implications for turbulence modulation in twophasc flows. Int. J. Multiphase Flow 24, 693720.

Bonneton, P. \& Chomax, J. M. (1992). Instabilitics of the wake generated by a spherc. C. R. Acad. Sci. Paris 314 (II), 10011006.

BoyD, J. P. (1989). Chebyshev and Fourier Spectral Methods. Springer Verlag.

Brown, D. L., CorTez, R., \& Minion, M. L. (2001). Accurate projection methods for the incompressible Navier Stokes equations. J. Compul. Phys. 168, 464499.

Brown, P. P. \& LAwler, D. F. (2003). Sphere drag and settling velocitics revisited. J. Environmental Engineering 129 (3), 222 230.

Canuto, C., Quarteroni, M. Y., \& ZANG, T. A. (1988). Spectral Methods in Fluid Dynamics. Springer Verlag Berlin.

Chakraborty, P., Banacinandar, S., \& Adrian, R. J. (2004). On the rolatiomships between local vortex identification sehemes. Under consideration, J. Fluid Mech.

Chenci, M., Chew, Y. T., \& Luo, S. C. (2001a). Numcrical investigation of a. rotationally oscillating cylinder in mean flow. J. Fluids and Struc. 15, 9811007.

Cheng, M., Liu, G. R., \& Lam, K. Y. (2001b). Numorical simulation of flow past. a rotationally oscillating cylinder. Comput. Fluids 30, 365392.

Chol, S., Chol, H., \& Kang, S. (2002). Characteristics of flow over a rotationally oscillating cylinder. Phys. Fluids 14 (8), 2767--2777. 
Chong, M. S., Perry, A. E., \& Cantwell, B. J. (1990). A general classification of three-dimensional flow ficlds. Phys. Fluids A 2 (5), 765777.

Chorin, A. J. (1968). Numerical solution of the Navier Stokes equations. Math. Comput. 22, 745762 .

Chou, M. (1997). Synchronization of vortex shedding from a cylinder under rotary oscillation. Comput. Fluids 26, 755774 .

Clift, R., Grace, J. R., \& Weber, M. E. (1978). Bubbles, Drops and Particles. Academic Pross.

Constantinescu, G. S. \& Squires, K. D. (2000). LES and DNS investigation of turbulent flow over a sphere. AIAA Paper 2000-0540.

Crow, S. C. (1970). Stability theory for a pair of trailing vortices. AIAA J. $8(12)$, 21722179 .

Cucitors, R., Qunirio, M., \& BAron, A. (1999). On the effectivoness and limitations of local criteria for the identification of a vortex. Eur. J. Mech. B 18 (2), 261282.

DAvies, J. M. (1949). 'The acroctynamies of goll balls. J. Appl. Phys. 20, 821828.

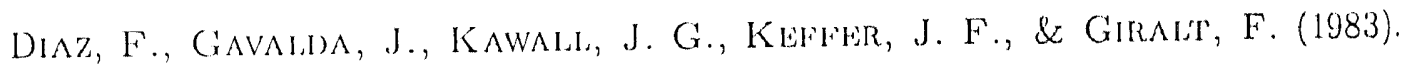
Vortex shedding from a spinning cylinder. Phys. Fluids 26 (12), 34543460.

DON, W. S. \& Solomonofr, A. (1995). Accuracy and speed in computing the Chebyshev collocation derivative. SIAM J. Sci. Comput. 16 (6), 12531268.

DubieF, Y. \& Delcayre, F. (2000). On coherent-vortex identification in turbulence. J. Turb. 1, 122. 
Filler, J. R., Marston, P. L., \& Mih, W. C. (1991). Response of the shcar laycr separating from a circular cylinder to small-amplitude rotational oscillations. J. Fluid Mech. 231, 481499.

FornberG, B. (1997). Comparison of finite difference and pscudospectral methods for convective flow over a sphere. Geophysical Research Letlers 24, 32453248.

German, M., Piomelli, U., Moin, P., \& Cabot, W. (1991). A dynamic subgridscale cddy viscosity model. Phys. Fluids A 3, 17601765.

Gottuleb, D. \& OrszaG, S. A. (1997). Numerical analysis of spectral methods: Theory and applications. Socicty for Industrial and Applied Mathomatics.

Gresho, P. M. \& SANi, R. L. (1987). On pressure boundary conditions for the incompressible Navier Stokes equations. Int. J. Numer. Melhods F'luids 7, 1111 1145.

Griffin, O. M. \& Hald, M. S. (1991). Review vortex shedeling lock-on and flow control in bluff body wakes. ASME J. Fluids Eng. 113, 526 537.

Hainvonfl, D. B. \& ZANG, T. (1979). The accurate solution of Poisson's ecpuation by expansion in Chebyshev polynomials. I. Compul. I'hys. 30, $1(67180$.

Hunt, J. C. R., Wray, A. A., \& MoIn, P'. (1988). Edlies, stroms, and convergence zones in turbulent flows. Contre for Turbulenee Rescarch Report, CrTR-S88, 193208.

Jeong, J. \& Hussain, F. (1995). On the identification of a vortex. J. Filuid Mech. 285, 6994 .

Johnson, T. A. \& Patel, V. C. (1999). Flow past a sphere up to a Reynolds number of 300. J. Fluid Mech. 378, 1970. 
Kang, S. \& Chor, H. (1999). Laminar flow past a rotating circular cylinder. Phys. Fluids 11 (11), 33123321.

KidA, S. \& MiURA, H. (1998). Identification and analysis of vortical structures. Eur. J. Mech. B 17 (4), 471488.

KıM, D. \& Chor, H. (2002). Laminar flow past a sphere rotating in the streamwise direction. J. Fluid Mech. 461, 365386.

Kim, H. J. \& Durbin, P. A. (1988). Observations of the frequencies in a sphere wake and of drag increase by acoustic excitation. Phys. Fluids 31, 32603265.

KIM, J. \& MoIN, P. (1985). Application of a fractional-step method to incompressible Navior Stokes equations. J. Comput. Phys. 59, 308323.

Ku, H. C., Hirsil, R. S., \& Taylor, T. D. (1987). A pscudospectral molhod for solution of the threc-dimensional Navior Stokes equations. J. Comput. Phys. 70, 439462.

Kurose, R. \& Komori, S. (1999). Drag and lift forces on a rotating sphere in a lincar shoar llow. J. Fluid Mech. 384, 183206.

LEWEKe, 'T'. The wake structure of a sphere placed in a uniform flow (private communication).

Leweke, T., Provansal, M., Ormiéres, D., \& Lebesciond, R. (1999). Vurtex dynamies in the wake of a sphere. Phys. Fluids $11(9), \mathrm{S} 12$.

Lu, X. \& S S ' $\mathrm{T}^{\prime} O$, J. (1996). A numerical study of flow past, a rotationally oscillating circular cylinder. J. Fluids and Struc. 10, 829849.

MacCOLl, J. H. (1928). Acrodynamies of a spinning sphere. J. Roy. Aero. Soc. 32, $777-798$. 
Magarvey, R. H. \& Bishop, R. L. (1961a). Transition ranges for threc-dimensional wakes. Can. J. Phys. 39, 1418-1422.

Magarvey, R. H. \& Bishop, R. L. (1961b). Wakes in liquid-liquid systems. Phys. Fluids $4,800-805$.

MAHFOUz, F. M. \& BADR, H. M. (2000). Flow structure in the wake of a rotationally oscillating cylinder. ASME J. Fluids Eng. 122, 290301.

Minne-Thomson, L. M. (1966). Theoretical Aerodynamics. St Martin's Press New York.

Mirtat, R. (1995). Study of flow past elliptic and circular cylinders using direct numerical simulation. PhD thesis University of Illinois at Urbana Champaign.

MitTal, R. (1999a). Planar symmetry in the unstcady wake of a splhere. $\Lambda I A \Lambda J .37$ (3), 388390 .

MitTAL, R. (1999b). A Fourier Chebyshev spectral collocation mothod for simulating flow past spheres and spheroids. Int. J. Numer. Methods Flutids 30, 921937.

MitTAL, R. \& BALAchinNDAR, S. (1996). Direct muncrical simmlation of flow past elliptic cylinders. J. Compul. Phys. 124, 351367.

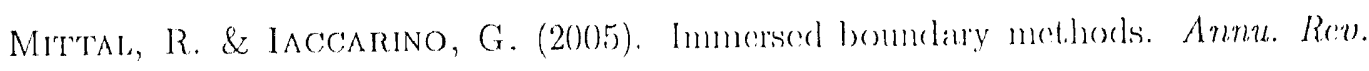
Fluid Mech. 37, 230) 261.

MirTsL, R. \& NAJJAR, F. M. (1999). Vortex dynamics in the sphere wake. AIAA Paper 99-3806.

MitTAL, R., Wilson, J. J., \& NaJjar, F. M. (2002). Symmetry properties of the transitional sphere wakc. AIAA J. $40(3), 579582$.

Mittal, S. \& Kumar, B. (2003). Flow past a rotating cylinder. J. Fluid Mech. 476, 303-334. 
Modi, V. J. \& AkUTSU, T. (1984). Wall confinement effects for spheres in the Reynolds number range of 30 2000. Transactions of ASME 106, 6673.

MoIN, P. (2001). Fundamentals of engineering numerical analysis. Cambridge University Press.

MoIN, P. \& KIM, J. (1985). The structure of the vorticity ficld in turbulent channel flow. Part 1. Analysis of instantancous ficlds and statistical corrclations. J. Fluid Mech. 155, 411464.

Morsi, S. A. \& Alexınder, A. J. (1972). An investigation of particle trajectorics in two-phase flow systems. J. Fluid Mech. 55, 193208.

Nakamurs, I. (1976). Stcady wake behind a sphere. Phys. Fluids 19 (1), 58.

Natarajan, R. \& Acrivos, A. (1993). The instubility of the stcady llow past sphercs and clisks. J. Fluid Mcch. 254, 323314.

NeW'TON, I. (1672). New theory of light and colours. Philos. Trans. R. Soc. London 1,67888 .

Niazmant), H. \& Rienksizbulute, M. (2003a). Surface effects on transient threcdimensional flows around rotating spheres at moderate Reynolds mumbers. Comput. Fluids $32,1405,1433$.

Ni^zMani), H. \& Rinksizizusu'T, M. (2003b)). Transiont throe-dimensional heat transfer from rotating spheres with surface blowing. Chem. Eng. Sci. 58, 3535 3554 .

Niazmand, H. \& Renksizbulutr, M. (2004). Heat transfor from a rolating sphere intcracting with a vortex. Inl. J. Heat Mass Transfer 47, 22692281.

Oesterlé, B. \& Dinh, B. (1998). Expcriments on the lift of a spinning sphere in a range of intermediate Reynolds numbers. Exps. Fluids 25, 16-22. 
Ongoren, A. \& Rockwell, D. (1988). Flow structure from an oscillating cylinder. Part 1. Mechanisms of phase shift and recovery in the near wake. J. Fluid Mech. $191,197223$.

Ormiéres, D. \& Provansal, M. (1999). Transition to turbulence in the wake of a spherc. Phys. Rev. Lett. 83, $80-83$.

OrszaG, S. A., Israeli, M., \& Deville, M. O. (1986). Boundary conditions for incompressible flows. J. Sci. Comp. 1, 75111.

Orzag, S. A. (1974). Fourier series on spheres. Month. Wealher Rev. 102, 5675.

Pan Y., TANaka, T., \& Tsuji, Y. (2001). Direct numerical simulation of particloladen rotating turbulent channel flow. Phys. Fluids 13 (8), 222230 ).

Pero't, J. B. (1993). An analysis of the fractional step mothod. J. Comprul. Phys. 108,5158 .

Perry, A. E. \& Lim, T. T. (1978). Coherent structures in coflowing jots and wakes. J. Flluid Mech. 88 (3), 451463.

P'Erre, A. E., Lim, T. T', \& Chong, M. S. (1980). The instantaneous velocity fiekds of coherent structures in coflowing jets and wakes. J. Flutd Mech. 101. (2), 243256.

Perrry, A. E. \& TAN, D. K. M. (1981). Simple threc-limensiomal vortex motions in collowing jets and wakes. J. Fluid Mech. 141, 197231.

Pertersson, N. A. (2001). Stability of pressure boundary conditions for Stokes and Navier Stokes equations. J. Comput. Phys. 172, 4070.

Pregnalato, C. J., Thompson, M. C., \& Hourigan, K. (2001). Flow transition in the wake of a rotating sphere. In Proc. 14 th $A F M C$. 
RAYLEIGH, L. (1877). On the irregular flight of a tennis ball. Messenger of Mathematics $7,14 \cdot 16$.

Robinson, S. K. (1991). Coherent motions in the turbulent boundary layer. Annu. Rev. Fluid Mech. 23, 601639.

Robinson, S. K., Kuine, S. J., \& Spalart, P. R. (1989). A revicw of quasicoherent structures in a numerically simulated turbulent boundary layer. Tech. Mom. 102191 NASA.

Roos, F. W. \& Willmarti, W. W. (1971). Some experimental results on sphere and disk drag. AIA J. 9, 285291.

Roshko, A. (1954). On the development of turbulent wakes from vortex strects. NACA Report 1191.

Rubinow, S. I. \& KellesR, J. B. (1961). The transverse force on a spinning sphere moving in a viscous fluicl. J. Fluid Mech. 11, 447459.

SAK MMO'TO, H. \& HANIU, H. (1990). A study on vortex shedeling from spheres in a unilorm flow. AS'ME: J. Fluids Engng 112, 386392.

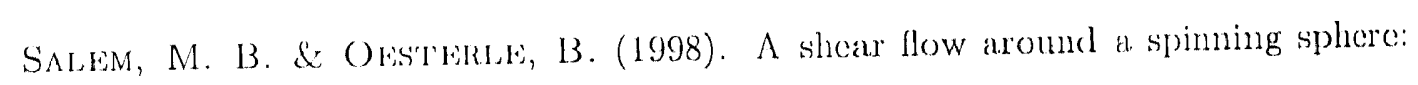
numerical study at moderate Reynolds numbers. Inl. J. Mulliphase Flow 24, 563 585.

Schouveilez, L. \& Provansal, M. (2002). Self-sustained oscillations in the wake of a sphere. Phys. Fluids 14 (11), 38463854.

Sengupta, T. K. \& TALLA, S. B. (2004). Robins Magnus effoct: $\Lambda$ continuing saga. Current Science 86, 10331036.

Shiels, D. \& LeONARD, A. (2001). Investigation of a drag reduction on a circular cylinder in rotary oscillation. J. Fluid Mech. 431, 297 322. 
Shirayama, S. (1992). Flow past a spherc: Topological transitions of the vorticity ficld. AIAA J. 30 (2), 349-358.

Smits, A. J. \& Lim, T. T. (2000). Flow Visualization: Techniques and Examples. Impcrial Collcge Pross London.

Steiner, T. R. (1984). A study of turbulent wakes and the vortex formalion process. PhD thesis University of Melbourne.

Stojković, D., Breuer, M., \& Durst, F. (2002). Effect of high rotation rates on the laminar flow around a circular cylinder. Phys. Fluids 14 (9), 31603178.

Streetrt, C. L. \& Hussaini, M. Y. (1991). A numerical simulation of the appoarance of chaos in finite-length taylor couctte flow. Appl. Numer. Malh. 7, 1171.

Tanaka, T., Yamagata, K., \& Tsuji, Y. (1990). Experiment of fluid forces on a rotating sphere and spheroid. In Proc. 2nd KSME-JSME liquids Eng. Conf.

TANEDA, S. (1956). Experimental investigation of the wake behind a sphere at low Reynolds numbers. J. Phys. Soc. Japan 11, 1104.

TANEDA, S. (1978a). Visual observations of the flow past a circular cylinder performing a rotatory oscillation. J. Phys. Soc. Japan 45, 10:38 104:3.

TANEDA, S. (1978h)). Vistal observations of the flow past, a sphorere at reynolds numbers between $10^{-1}$ and $10^{6}$. J. Fluid Mech. 85, 187192.

TemAM, R. (1991). Remark on the pressure boundary condition for the projection mothod. Theorel. Compul. Fluid Dynamics 3, 181184.

Thompson, M. C., Leweke, T., \& Provansal, M. (20)1). Kincmatics and dynamics of sphere wake transition. J. Fluids and Struc. 15, 575585.

Tokumaru, P. T. \& Dimotakis, P. E. (1991). Rotary oscillation control of a cylinder wakc. J. Fluid Mech. 224, 77-90. 
Tomboulides, A. G. (1993). Direct and large-eddy simulation of wake flows: Flow past a sphere. $\mathrm{PhD}$ thesis Princeton University.

Tomboulides, A. G. \& OrszaG, S. A. (2000). Numerical investigation of transition and weak turbulent flow past a sphere. J. Fluid Mech. 416, 45 73.

Torobin, L. B. \& Gıuvin, W. H. (1960). Fundamental aspects of solids-gas flow, part IV : The effects of particle rotation, roughness and shape. Can. J. Chem. Eng. 38,142153 .

Tsuji, Y., Moriknwa, Y., \& Mizuno, O. (1985). Experimental measurements of the Magnus force on a rotating sphere at low Reynolds numbers. Trans. ASME 107, 484488 .

UMSCheid, L. \& SANKAR-IRAO, M. (1970). Further lests of a grid system for global numerical prediction. Month. Weather Rev. 99,686 690.

Williamson, C. H. K. (1996). Vortex dynamies in the cylinder wake. Annu. Rev. Fluid Mech. 28, 477 539.

You, C. F., QI, H. Y., \& Xu, X. C. (2003). Lift force on rotating sphere at low Reynoleds numbers and high rotational speeds. ACTA Mechanica Sinica 19 (4), 300307.

ZHou, J., AdrinN, R.. J., \& BALACHANDAR, S. (1996). Autogeneration of nour-wall vortical structures in chamnel flow. Phys. Fluids 8 (1), 288290.

Zhou, J., Adrian, R. J., Balachandar, S., \& Kendali, T. M. (1999). Mochanisms for gencrating coherent packets of hairpin vortices in channel flow. J. Fluid Mech. $387,353396$. 


\section{Appendix A}

\section{Fourier series on spheres}

On a standard spherical polar coordinate grid, the lines of constant polar angle or azimuthal angle $(\phi)$ converge to single points on the polar axis. This has two important consequences, which recuire careful treatment when differential equations are solved numerically. These are the so-called 'pole problem' and the 'pole parity problem'. The pole problem manifests itself as a restrictive time-stepping limit. The numerical technique used to alleviate this problem is discussed in \$2.2.1. The pole parity problem refors to the tangential and azimuthal components of a vector field undergoing a stepjump sign change when the poles are crossed. The tangential and azimuthal derivatives of a scalar and of a radial component of a vector may also be discontinuous across the poles.

To arrive at acreptable expansions, the approach used by Boyel (1989) is followed. The discussion that follows considers the Fourier expansions only on a spherical shell, since the inclusion of the radial expansion presents no added complexity. Physically, a continuous variable defined over a spherical shell must be periodic over $2 \pi$ in both the $\theta$ and $\phi$ coorclinates. In this case, it would appear natural to use a general Fourier expansion in both directions. However on a sphere lines of constant longitude and latitude intersect at two points not one, as is the case for a rectangular domain. As a result, the Fourier modes in one direction may conflict with certain Fouricr modes in the other direction and only certain combinations of the basis functions are permissible.

We start by considering a double periodic Fouricr expansion. Then by examining 
the behavioral boundary conditions that must be satisfied in the vicinity of the poles, the incompatible terms in the basis functions are omitted. The expression for a scalar function, $S(\theta, \phi)$, is considered first. The expansion for a vector ficld, $V(\theta, \phi)$, is more complicated because it is generally multi-valued at the pole and so its discussion is left to later.

\section{A.1 Basis functions for a scalar variable}

The expansion of a scalar function, as a double Fourier series can be expressed as

$$
S(\theta, \phi)=\sum_{m=0}^{\infty} \sum_{k=0}^{\infty} \widehat{s_{m k}} e^{\mathrm{i} m \theta} e^{\mathrm{i} k \phi}
$$

where $m$ and $k$ are the tangential and azimuthal wavenumbers and $\widehat{s m k}$ are the Fourier expansion coefficients. Equation (A.1) can also be written in the expanded form

$$
S(\theta, \phi)=\sum_{k=0}^{\infty} \sum_{m=0}^{\infty} \widehat{s_{m k}}\{\cos (m \theta)+\mathrm{i} \sin (m \theta)\}\{\cos (k \cdot \phi)+\mathrm{i} \sin (k \phi \phi)\},
$$

or after grouping the ' $\theta$ ' terms, as

$$
S(\theta, \phi)=\sum_{k=0}^{\infty} a_{k}(\theta) \cos (k \phi)+\sum_{k=1}^{\infty} b_{k}(\theta) \sin (k \phi) .
$$

The general form of the functions $a_{k}(\theta)$ and $b_{k}(\theta)$ must be reduced lo be compatible with both oven and odd azimulhal modes. Figure $\Lambda .1$ presents schematics of lypical even and oxld azimuthal modes in the vieinity of a pole. $\Lambda$ plane that is tangente to the spherienl shell an, the pole is shown as a transhucent, circular disk. The nodal lines, which are dedined by the intersection of the functions $a_{k}(\theta)$ and $b_{k}(\theta)$ and the circular disk, are also shown. The cases shown are even azimuthal mode: $k=2$ and odd mode $k=3$. These mode shapes apply to both the functions $a_{k}(\theta) \cos (k \cdot \phi)$ and $b_{k}(\theta) \sin (k \phi)$, the only difference is that the datum from which $\phi$ is measure is shifted by $\pi / 2$.

For the odd azimuthal wavenumber shown in figure A.1(b), compatibility requires that the functions $a_{k}(\theta)$ and $b_{k}(\theta)$ change sign across the pole, that is, to avoid a discontinuity at the pole they must have odd parity. Since differentiation is a parity 
reversing operation, it follows that all cven derivatives of $a_{k}(\theta)$ and $b_{k}(\theta)$ must also have odd parity across the pole. A Fourier sine series and its even derivatives naturally satisfics this constraint. For a Fourier cosine series this constraint is satisfied only for the trivial solution. All odd derivatives of $a_{k}(\theta)$ and $b_{k}(\theta)$ will have cven parity across the pole. Since $\theta$ is measure away from the pole, as the pole is cross the sign of $\theta$ and all derivatives with respect to $\theta$ will change sign. For an even function, with a non-zero value at the pole, a sign change is only possible if there is a discontinuity at the pole. His discontinuily does not destroy the spectral convergenee of the series. In fact, the derivative of a scalar field with respect to $\theta$ behaves like a vector ficld. This point is claborated on in $\S \Lambda .2$, where an acceptable expansion for a vector field is deduced. Hence, for even azimuthal modes, the functions $a_{k}(\theta)$ and $b_{k}(\theta)$ will be smooth across the pole and exponentially convergent, if the functions comprise only the terms of a Fouricr sine serics.

Considering now the even azimuthal mode, figure A.1(a) shows that the functions $a_{k}(\theta)$ and $b_{k}(\theta)$ must be symmetric, or have even parity, across the poles. It follows that all even derivalives of the functions $a_{k}(\theta)$ and $b_{k}(\theta)$ must also have even parity. All odd derivatives of the functions $a_{k}(\theta)$ and $b_{k}(\theta)$ must be antisymmetric across the pole and therefore must have a zoro at, the pole. For odd azimuthal modes, it was argued that for an antisymmetric function, a Fourier sine series is exponentially convergent. For the even azimuthal modes, it is all odd derivative that are antisymmetric. In this case it is all odel derivatives that, should comprise only the terms of a Fourier sine series. If follows that even derivatives of $a_{k}(\theta)$ and $b_{k}(\theta)$ and the functions themselves must comprise the sum of a Fourier cosine series. In contrast to odd azimuthal modes, here it is even derivatives that may be discontinuous at the poles and must be treated as vectors.

In summary, a scalar function, $S(\theta, \phi)$, that is non-singular across the poles can be 
expressed by the exponentially convergent series

$$
S(\theta, \phi)=\left\{\begin{array}{lll}
\sum \widehat{s_{m k}} \cos (m \theta) e^{\mathrm{i} k \phi} & \text { even } & k \\
\sum \widehat{s_{m k}} \sin (m \theta) e^{\mathrm{i} k \phi} & \text { odd } & k
\end{array}\right.
$$

This restricted expansion is suitable for scalar ficlds such as pressurc. It is also suitable for radial components of a vector field and its radial derivatives, since these do not change sign as they cross the pole.

(a)

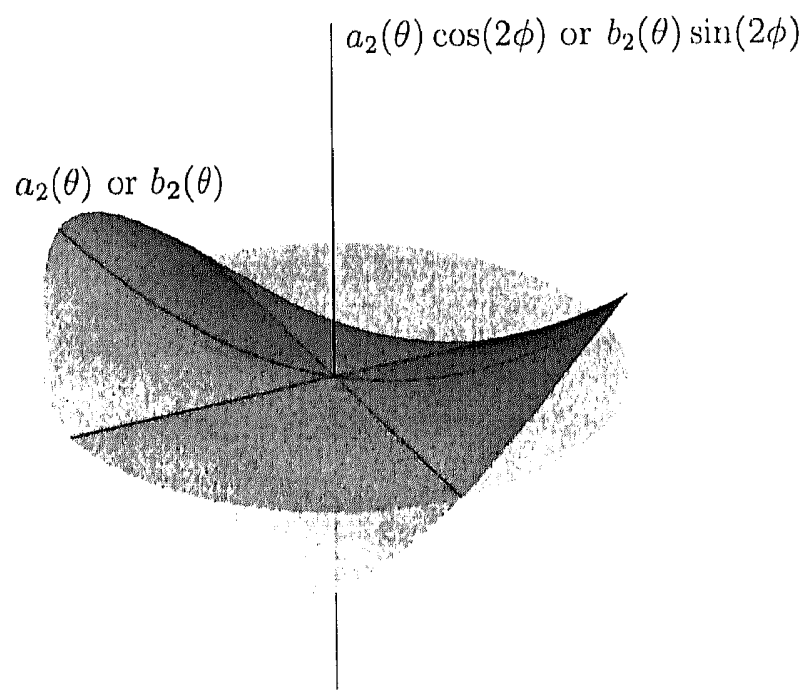

(b)

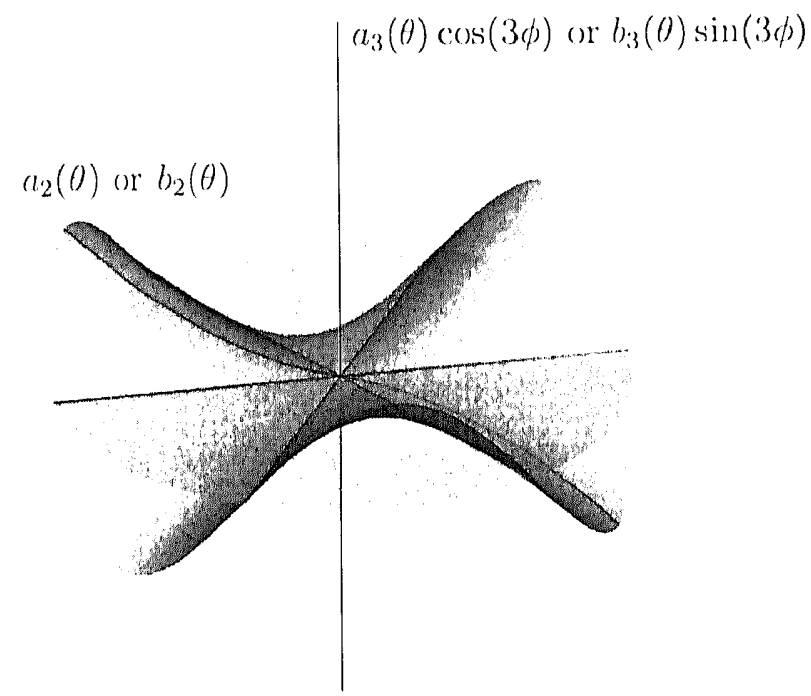

Figure A.1: Schematic of typical even and odd azimuthal modes in the vicinity of a pole: (a) cven azimuthal mode $k=2,(b)$ odd azimuthal mode $k=3$. 


\section{A.2 Basis functions for a vector variable}

A vector function that is smooth in cartesian coordinates may be discontinuous or may have discontinuous derivatives at the poles when transformed into a spherical coordinate system. In fact, this is the case for any vector function that is non-zero at the poles. $\dagger$ Boyd (1989) illustrates this graphically by transforming a continuous cartesian velocity field into spherical coordinates and observing the behavior of the spherical components across the pole. Consider, for example, a uniform flow of magnitude $U_{\infty}$, directed along the $x$-axis. In cartesian coordinates this field comprises even mode zero, while in spherical coordinates it comprises the odd mode, $k=1$, variation:

$$
\left\{\begin{array}{l}
u_{r} \\
u_{\theta} \\
u_{\phi}
\end{array}\right\}=U_{\infty}\left\{\begin{array}{c}
\sin (\theta) \cos (\phi) \\
\cos (\theta) \cos (\phi) \\
-\sin (\phi)
\end{array}\right\} .
$$

Figure A.3(a) presents these spherical velocity components in the vicinity of the downstream pole of the sphere (about $\theta=0)$. The radial velocity component, $u_{r}$, has odd parity across the pole. This behavior is consistent with that found for a scalar ficld when the azimuthal wavenumber is odd. Wo find that the radial velocity transforms like a scalar and therefore a satisfactory Fouricr expansion for the radial velocity takes the form of ecpuation $\Lambda .4$. The tangential and azimuthal velocity components behave differently moler the transformation. Figure A.3 $(b, c)$ shows that both $u_{0}$ and $u_{\phi}$ are discontimuous acress the pole. If a Fourier sine series is included in the function $a_{k}(\theta)$ and $b_{k}(\theta)$, Gibbs phenomena will result in the vicinity of the pole and the convergence of the series is expected to be slow over the whole domain. To avoid this problem, the expansions for $a_{k}(\theta)$ and $b_{k}(\theta)$ must have even parity. All even derivatives of $a_{k}(\theta)$ and $b_{k}(\theta)$ are also discontinuous across the pole, while all odd derivatives smoothly go to zero at the pole. A Fourier cosine series ensures the correct behavior for all derivatives. Hence, for odd azimuthal wavenumber, the functions $a_{k}(\theta)$ and $b_{k}(\theta)$ should comprise the sum of a Fouricr cosine series.

† It is important to highlight that this is not a physical discontinuity, but rather a result of the coordinate system itself. 
As a second example, consider a linear shear flow, which in cartesian coordinates is given by

$$
\left\{\begin{array}{l}
u_{x} \\
u_{y} \\
u_{z}
\end{array}\right\}=U_{\infty}\left\{\begin{array}{l}
y \\
0 \\
0
\end{array}\right\}=U_{\infty}\left\{\begin{array}{c}
r \sin (\theta) \sin (\phi) \\
0 \\
0
\end{array}\right\}
$$

Transformation to spherical coordinates gives

$$
\left\{\begin{array}{l}
u_{r} \\
u_{\theta} \\
u_{\phi}
\end{array}\right\}=\frac{1}{2} U_{\infty} r\left\{\begin{array}{c}
\sin ^{2} \theta \sin 2 \phi \\
\sin \theta \cos \theta \sin 2 \phi \\
\sin \theta(\cos 2 \phi-1)
\end{array}\right\} .
$$

In this case, a cartesian ficld with a mode one variation, when recast into spherical coordinates, results in an cven azimuthal mode, $k=2$, variation. The transformation has resulted in an interchange of even and odd azimuthal modes this is a general property of the in-plane vector ficlds on a sphere. Figure $\Lambda .2$ shows the cartesian velocity component, $u_{x}$, in the vicinity of the pole. The spherical velocity components for this flow are shown in figure A.4. The radial velocity has even parity across the

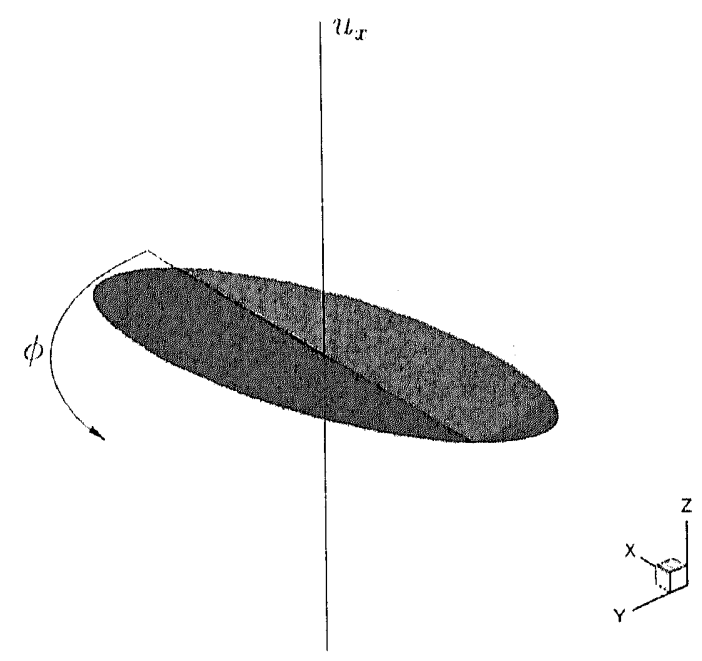

Figure A.2: Cartesian velocity component in the vicinity of the pole for the shear flow $u_{x}=U_{\infty} y$.

pole. Again the behavior of the radial velocity is consistent with that of a scalar field. The tangential and azimuthal velocitics smoothly go to zero at the pole. All even derivatives will also go smoothly to zero, while all odd derivatives are discontinuous 
across the polc. In this casc an acceptable cxpansion for even azimuthal modes is the sum of a Fourier sine series.

In summary, a function which behaves like a vector function, $V(\theta, \phi)$, be can be expressed by the exponentially convergent scrics

$$
V(\theta, \phi)=\left\{\begin{array}{lll}
\sum \widehat{v_{m n}} \sin (m \theta) e^{\mathrm{i} k \phi} & \text { even } & k \\
\sum \widehat{v_{m n}} \cos (m \theta) e^{\mathrm{i} k \phi} & \text { odd } & k
\end{array}\right.
$$

The difference between this expansion and cquation A.4 for a scalar function is the reversal of the parity in the tangential expansion functions. 


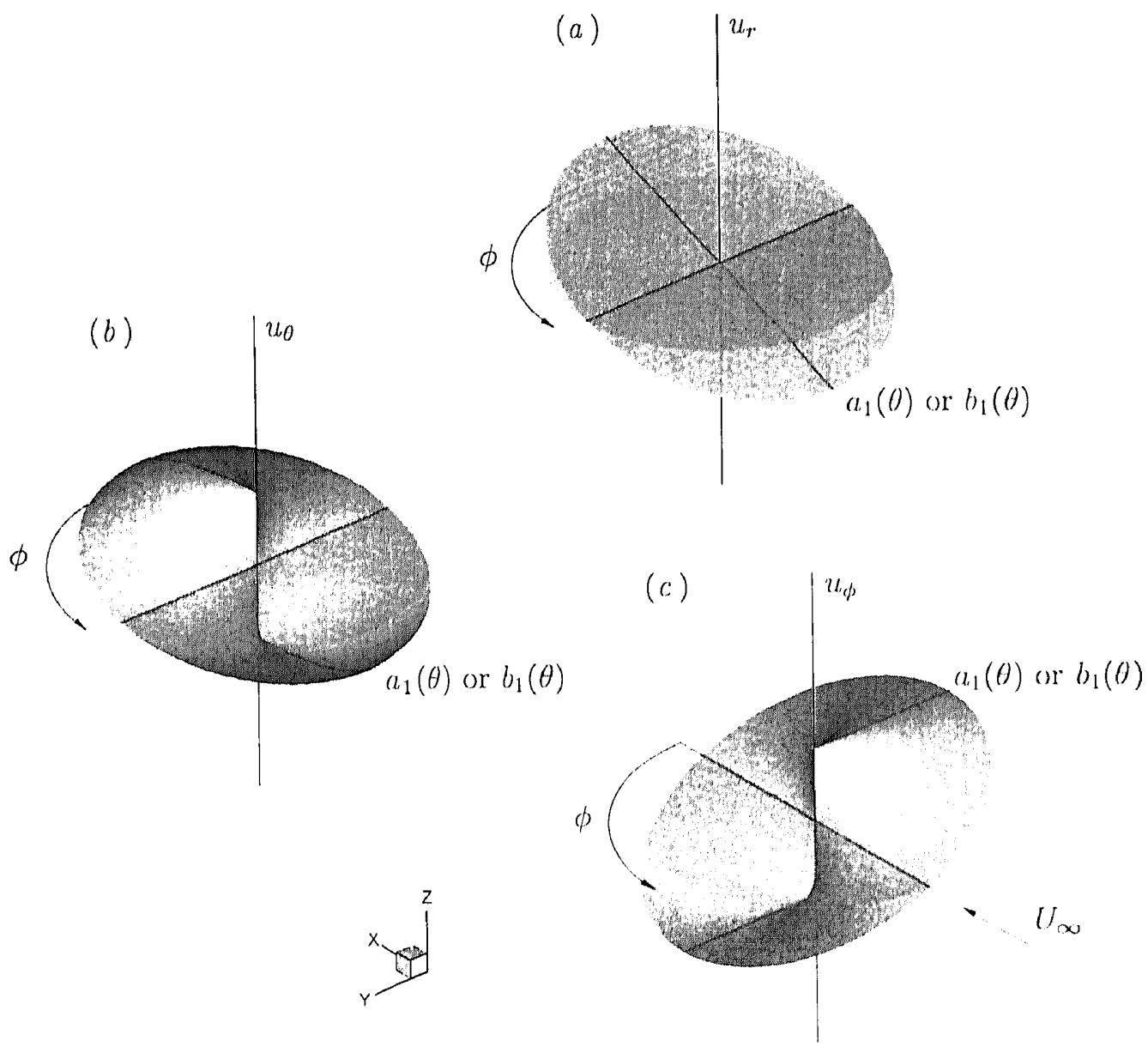

Figure 4.3: Schematic of the spherical velocity components in the vicinity of the pole for the cartesian velocity ficld $u_{x}=U_{\infty}$ : $(a)$ radial velocity, $u_{r},(b)$ tangential volocity, $u_{\theta},(c)$ azimuthal velocity, $u_{\phi}$. A cartesian velocity fickl of even wavenumber is transformed into polar spherical velocity components of odd azimuthal wavemumber. 


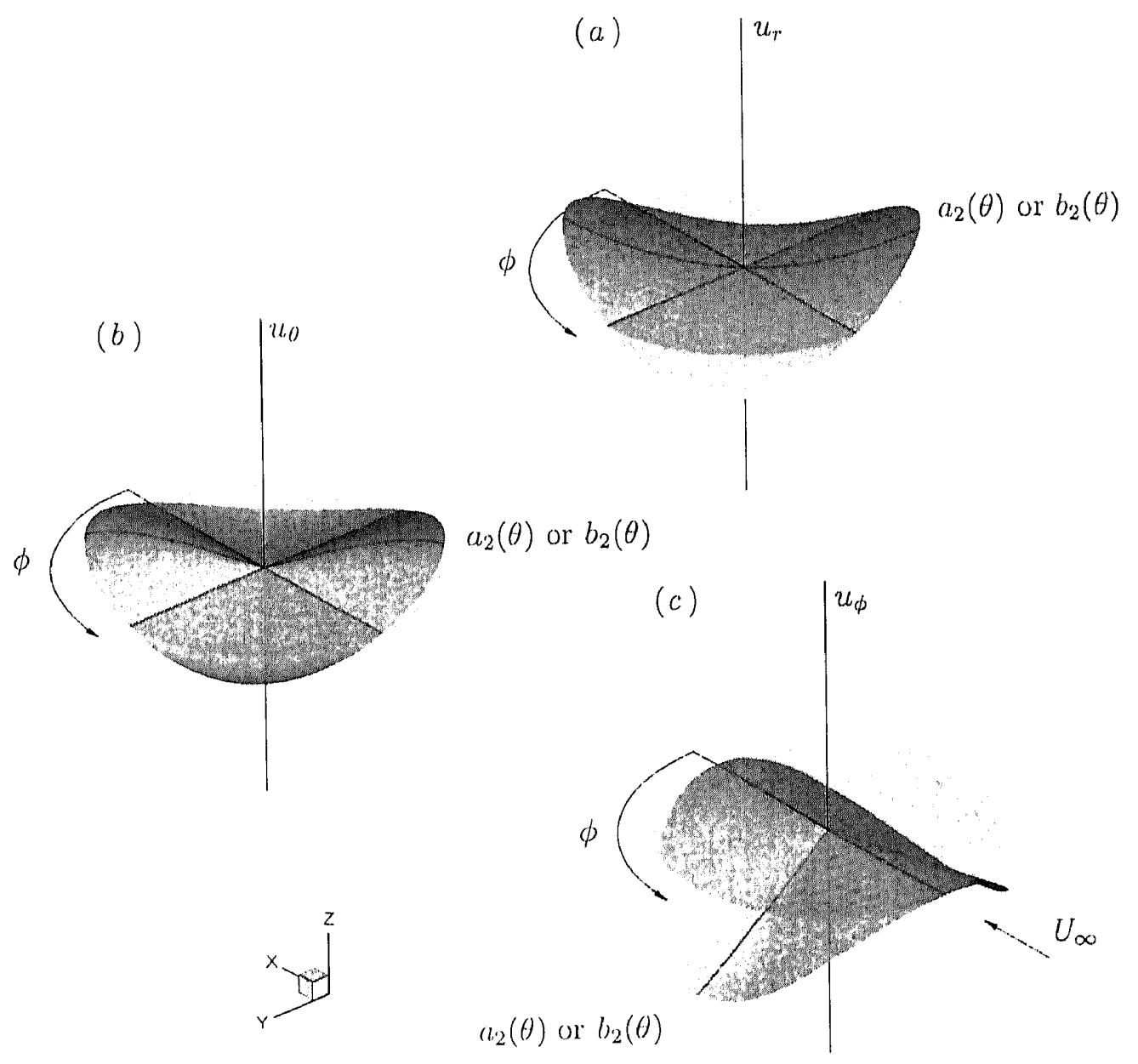

Figure A.4: Schematic of the spherical velocity components in the vicinity of the pole for the cartesian velocity ficld $u_{x}=U_{\infty} y:$ (a) radial velocity, $u_{r},(b)$ tangential velocity, $u_{\theta},(c)$ azinuthal velocity, $u_{\phi}$. A cartesian velocity field of odd wavenumber is transformed into polar spherical velocity components of even azimuthal wavenumber. 


\section{Appendix B}

\section{Instantaneous streamline patterns on the $(x, z)$-plane}

Figure B.1 and B.2 present the near-wake stroamline patterns on the $(x, z)$-plane for $R e=250$ and $R e=300$, respectively. The patterns are constructed from the velocity field resolved onto the $(x, z)$-plane and do not represent true streamlines. For those cascs that exhibit a periodic wake, the time instant shown coincicles with the lateral lift force being at a local minimum. These instantancous palterns were obtained by numerically integrating the instantaneous velocity field in pseudo-time. 


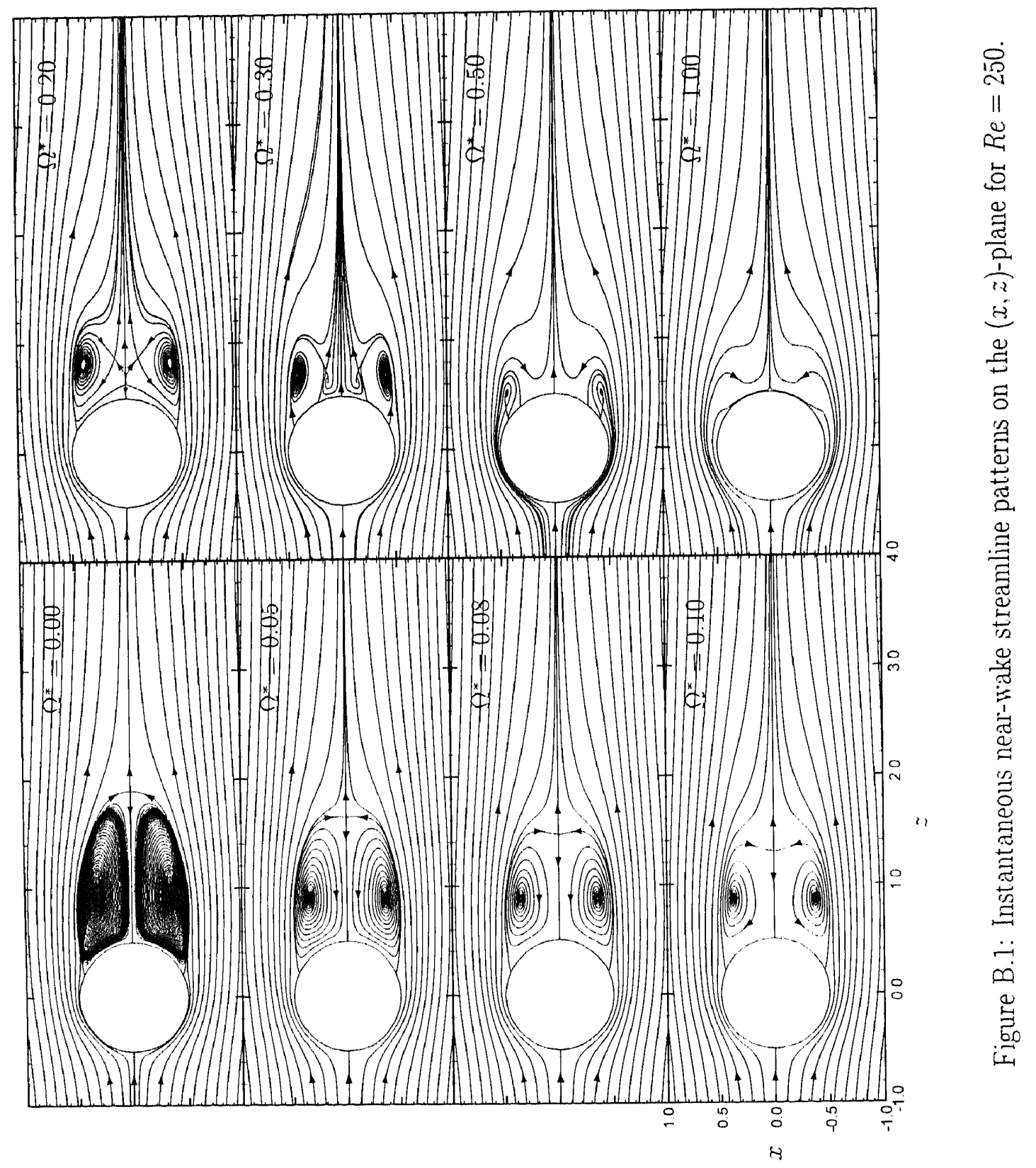




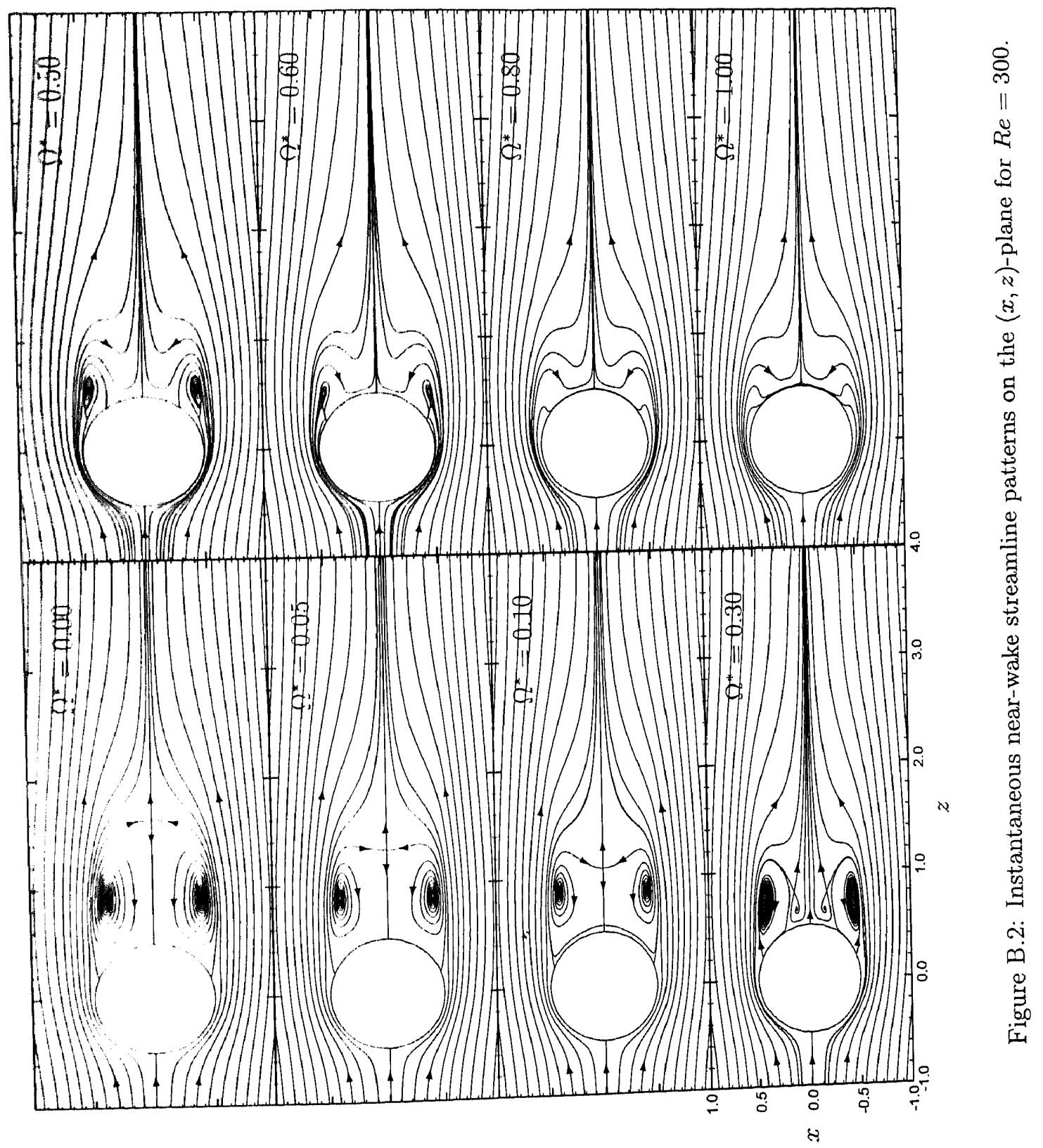




\section{Appendix $\mathbf{C}$}

\section{The velocity boundary condition on a spinning sphere}

In this appendix the velocity boundary condition appropriate at the surface of a spinning sphere is derived for the cylindrical coordinate systems employed by Kuruse \& Komori (1999) and Niazmand \& Renksizbulut (2003a). These are shown to be different to the boundary conditions actually prescribed in these studies. This excreise serves to explain the discrepancy reported in chapter 5 between the findings presented in these studies and the present study.

(a)

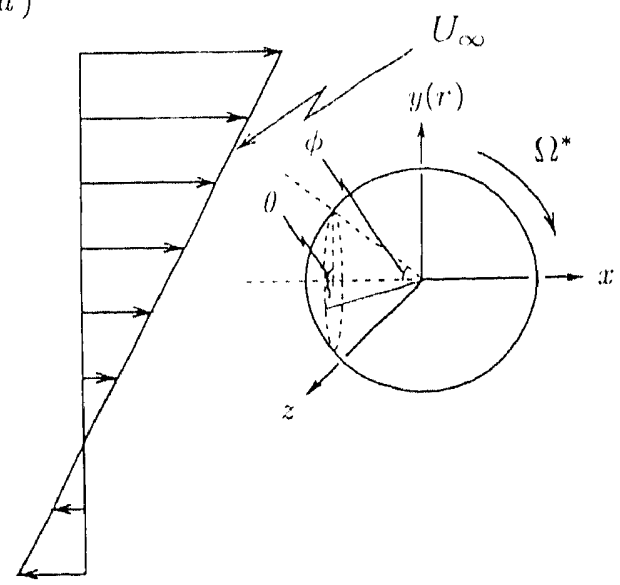

(b)

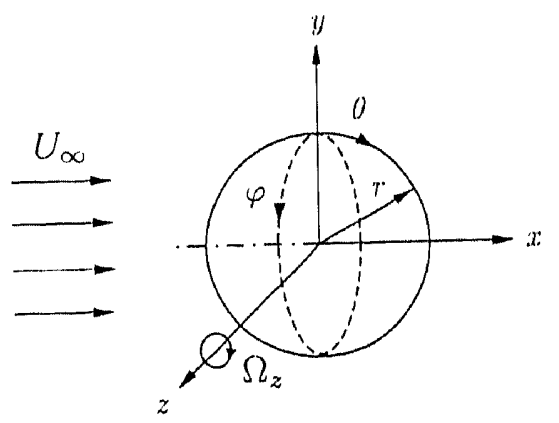

Figure C.1: Coordinate system employed by: (a) Kurose \& Komori (1999); (b) Niazmand \& Renksizbulut (2003a)

The coordinate systems used by Kurose \& Komori and Niazmand \& Renksizbulut are presented in figure C.1. Both studies use identical cartesian coordinate systems, 
while the spherical coordinate systems use a different nomenclature. Here the velocity boundary condition for the coordinate system used by Kurose \& Komori is deriverl. It is then a simple matter to replace the nomenclature used by Kurose \& Komori for the polar angles, with that used by Niazmand \& Ronksizbulut.

For the coordinate system employed by Kurose \& Komori, the transformation from a unit vector in cartesian coordinates, $\left\{\hat{e}_{\boldsymbol{x}}, \widehat{e}_{y}, \widehat{e}_{z}\right\}$, to spherical coordinates, $\left\{\hat{e}_{\boldsymbol{r}}, \widehat{e}_{0}, \widehat{e}_{\phi}\right\}$, may be written in matrix form as

$$
\left\{\begin{array}{l}
\widehat{e}_{r} \\
\widehat{e}_{\theta} \\
\widehat{e}_{\phi}
\end{array}\right\}=\left[\begin{array}{ccc}
-\cos \phi & \sin \phi \cos \theta & \sin \phi \sin \theta \\
0 & -\sin \theta & \cos \theta \\
\sin \phi & \cos \phi \cos \theta & \cos \phi \sin \theta
\end{array}\right]\left\{\begin{array}{l}
\hat{e}_{\boldsymbol{x}} \\
\widehat{e}_{y} \\
\hat{e}_{z}
\end{array}\right\}
$$

while the transformation from cartesian coordinates to cylindrical coorclinates, $\left\{\widehat{e}_{x}, \widehat{e}_{r}, \widehat{e}_{o}\right\}$, lakes the form

$$
\left\{\begin{array}{l}
\widehat{e}_{x} \\
\widehat{e}_{r} \\
\widehat{e}_{\theta}
\end{array}\right\}=\left[\begin{array}{ccc}
1 & 0 & 0 \\
0 & \cos \theta & \sin \theta \\
0 & -\sin \theta & \cos \theta
\end{array}\right]\left\{\begin{array}{l}
\widehat{e}_{x} \\
\widehat{e}_{y} \\
\widehat{e}_{z}
\end{array}\right\}
$$

For a splece rotating about the negative $z$-axis, the angular velocily vector in cartesian coordinates is $\Omega=-\Omega \widehat{e}_{\boldsymbol{z}}$. By applying the transformation given by egualion $(C .1)$, the angular velocity vector in spherical coordinates becomes

$$
\left.\left.\Omega=-\Omega\left(\sin \phi \sin \theta \hat{e}_{r}+\cos \theta\right) \hat{e}_{\theta}+\cos \phi\right) \sin \theta \hat{e}_{\phi}\right),
$$

and the non-rlinensional surface velocity vector is

$$
\left.u_{b}=-\frac{\Omega \times r}{U_{\infty}}=\frac{S a}{U_{\infty}}\left(\sin \theta \cos \phi \hat{e}_{0}-\cos \theta\right) \hat{e}_{\phi}\right)
$$

where a is the radius of the sphere. Using (C.1) to transtorm back to cartesian corordinates, the velocity vector becomes

$$
u_{b}=\frac{S a}{U_{\infty}}\left(\cos \theta \sin \phi \widehat{e}_{x}+\cos \phi \widehat{e}_{y}\right)
$$

Finally, by applying the transformation give by C.2 and introducing the non-dimensional angular specd $\Omega^{*}=\Omega a / U_{\infty}$, the velocity vector in cylindrical coordinates becomes

$$
u_{b}=\Omega^{*}\left(\cos \theta \sin \phi \widehat{e}_{x}+\cos \theta \cos \phi \widehat{e}_{r}-\sin \theta \cos \phi \widehat{e}_{\theta}\right)
$$


This expression is not in agrecment with equations (2.12) (2.14) of Kurose \& Komori, where the velocity at the sphere surface,

$$
u_{b}=V_{x} \widehat{e}_{x}+V_{r} \widehat{e}_{r}+V_{\theta} \widehat{e}_{\boldsymbol{\theta}}
$$

is given as

$$
\begin{aligned}
& V_{x}=\Omega^{*} \cos \theta \sin \phi, \\
& V_{r}=\Omega^{*} \cos \theta \cos \phi, \\
& V_{0}=0 .
\end{aligned}
$$

For the coordinale system of Niazmand \& Renksizbulut, the polar angles $(\phi, \theta)$ in equation $(C .6)$ are replaced by $(\theta, \varphi)$, such that the velocity vector in cylinclrical coordinates becomes

$$
u_{b}=\Omega \Omega_{z}\left(\cos \varphi \sin \theta \widehat{e}_{x}+\cos \varphi \cos \theta \widehat{e}_{r}-\sin \varphi \cos \theta \widehat{e}_{\theta}\right) .
$$

This expression is not, in agreement with ecplation (8) of Niazmand \& Ronksizbulut, where the velocity vector at the surface,

$$
u_{b}-u \widehat{e}_{x}+v \widehat{e}_{r}+w \widehat{e}_{\theta},
$$

is given as

$$
\left.u-\Omega_{z} \cos \theta \sin \phi, \quad v=\Omega_{z} \cos \theta \cos \phi, \quad u\right)=0 .
$$

